\title{
Real-Time Data Visualisation in Collaborative Virtual Environment for Emergency Management by
}

Pan Wang

Submitted in total fulfilment of the requirements of the degree of Doctor of Philosophy

Department of Infrastructure Engineering

The University of Melbourne

September 2011 


\begin{abstract}
A Collaborative Virtual Environment (CVE) is a shared virtual environment used for collaboration and interaction of many participants that may be spread over large distances. Although CVE has been widely used in emergency management, especially for education, training and assessment, there are some drawbacks and challenges in existing CVEs: 1. The authenticity of emergency simulation in CVE still needs improvement. 2. Delivery of up-to-date information cannot be guaranteed in currently available CVE. 3. The problems with usability of CVE are common, including the user collaboration and scenario creation. A review of the current literature reveals that, until now, these problems have not been well addressed.

This thesis focuses on the design and implementation of a prototype system that facilitates emergency management via a Collaborative Virtual Environment using real-time spatial information. The system, Spatial Information Exploration and Visualisation Environment - Virtual Training System or SIEVE-VTS, was developed based on a game engine. It automatically integrates real-time data from multiple online sources, then models and simulates emergency incident scenarios using such data.

The prototype system provides the capability of simulating dynamic scenarios in the virtual environment, extends the traditional technique of real-time data collection from 2D maps to the 3D virtual environment, manipulates spatial information efficiently and effectively, and enhances collaboration and communication between users. It improves the processes and outcomes of emergency management by increasing engagement and supporting decision making of potential users, including first responders, emergency managers and other stakeholders.
\end{abstract}




\section{Declaration}

This is to certify that

1. the thesis comprises only my original work towards the $\mathrm{PhD}$,

2. due acknowledgement has been made in the text to all other material used,

3. the thesis is less than 100,000 words in length, exclusive of tables, maps, bibliographies and appendices.

Pan Wang

September 2011 


\section{Acknowledgements}

First and foremost, I would like to express my gratitude to my supervisor, Professor Ian Bishop, for his support and guidance throughout each phase of my $\mathrm{PhD}$ candidature. He provided me early ideas and made incisive comments and constructive suggestions on my research. Without his supervision, this thesis would not have been completed. I would also like to thank Dr Christian Stock for his supervise in the first two years.

I also acknowledge Dr. Alison Kelly and Assoc. Prof. Stephan Winter at University of Melbourne for their invaluable suggestions in this study. Appreciation also goes out to Dr Gangjun Liu in the Department of Mathematical and Geospatial Sciences at RMIT. He provided excellent advice and constructive comments on my research. I would also like to thank my colleague students in CRC-SI, particularly Haohui Chen, Yiqun Chen, Marcos Nino-Ruiz and Subbash Sharma, who provided help on data collection and programming in my research, and formed a fun time outside research.

I am thankful to collaborators from DSTO for testing the system and providing feedback, particularly Mark Hellett, Ping Cao, Bernard Kachoyan and Tristan Cooper. I would also like to thank people from DIGO and Bureau of Meteorology providing data and models for my research.

I recognize that this research would not have been possible without the financial assistance of CRC for Spatial Information and the University of Melbourne, for they provided me with scholarships and travel funds to pursue doctoral studies and attend conferences. The CRC-SI also provided a good working environment and connections to a range of organisations and people which made valuable collaboration, data access and testing possible. I also extend my gratitude to office staff, including Pauline Woolcock and Jane Inall, for all the instances in which their assistance helped me along the way. Appreciation also goes to Miss Lillian Gabra Fam for proof reading of my work.

I would like to thank my family for their support, dedication and love at all times and in particular, I must acknowledge my wife and best friend, Ting $\mathrm{Hu}$, without whose love, understanding and encouragement, I would not have finished this thesis. 


\section{Table of Contents}

Chapter 1 Introduction .................................................................... 1 -

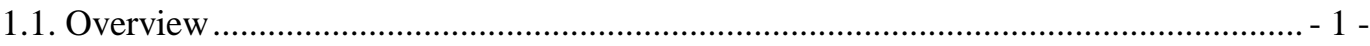

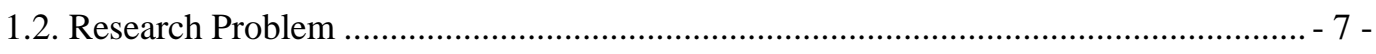

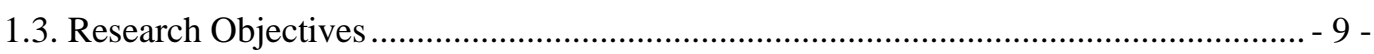

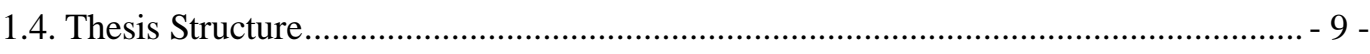

Chapter 2 Literature Review .......................................................... 13 -

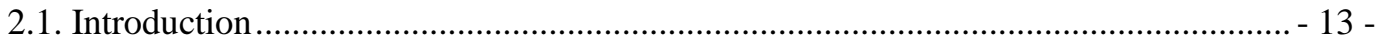

2.2. Emergency Monitoring for Response ……………............................................... 15 -

2.2.1. Emergency monitoring introduction ......................................................... - 15 -

2.2.2. Emergency monitoring techniques................................................................ 16 -

2.3. Emergency Training and Implementation Tools ....................................................... - 18 -

2.3.1. Emergency training introduction............................................................... 18 -

2.3.2. Virtual emergency training and related tools............................................... - 20 -

2.4. Emergency Management with GIS .......................................................................... 24 -

2.5. Spatial Information Visualisation in a 3D Environment .............................................. 26 -

2.5.1. 3D functionalities in GIS software ........................................................... - 26 -

2.5.2. 3D visualisation software ............................................................................ 27 -

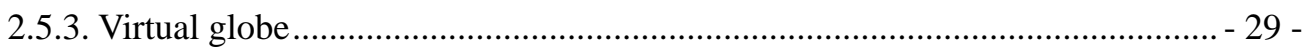

2.5.4. Current CVE development .......................................................................... 32 -

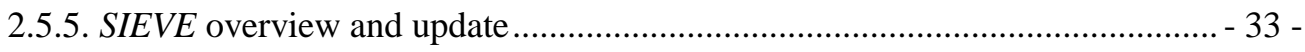

2.6. Real-Time Data and Related Technologies .............................................................. 36 -

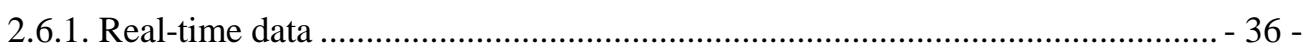

2.6.2. Real-time data collection and distribution......................................................... - 38 -

2.6.3. Real-time data modelling and visualisation ..................................................... - 41 -

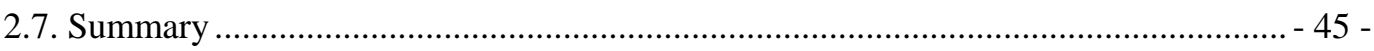

Chapter 3 System Design and Methodology ................................. 47 -

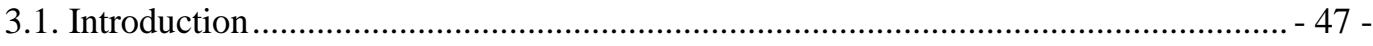

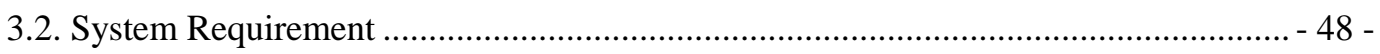

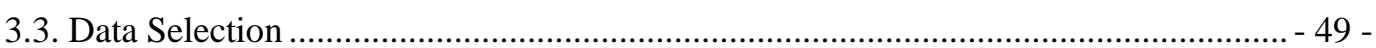

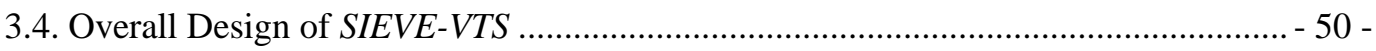

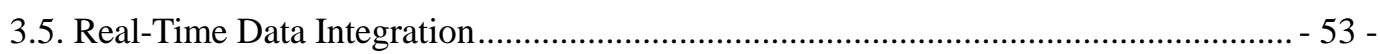

3.5.1. Real-time data integration using Web scraping............................................... - 56 -

3.5.2. Collecting real-time data using mashup technology .......................................... - 62 -

3.5.3. Collecting real-time data using mobile device .................................................. - 67 -

3.6. Real-Time Data Translation ................................................................................................

3.6.1. Location translation........................................................................................... 72 -

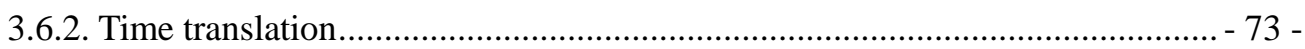

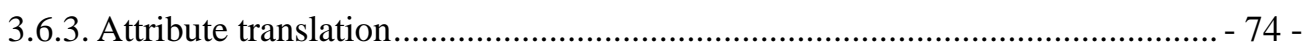

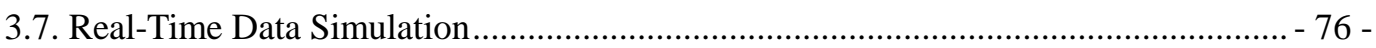




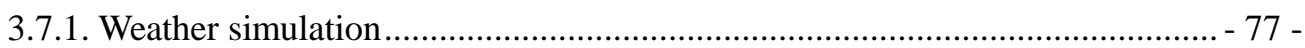

3.7.2. Oceanographic information simulation .......................................................... 85 -

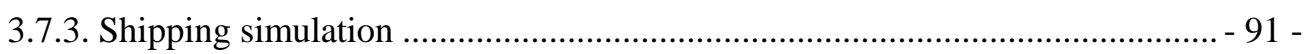

3.7.4. Incident location and status simulation ........................................................... - 99 -

3.8. Spatial Interpolation for Real-Time Data ................................................................ 101 -

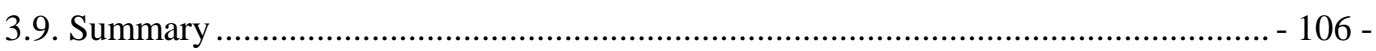

Chapter 4 Emergency Scenario Simulation Using SIEVE-VTS ........- 109 -

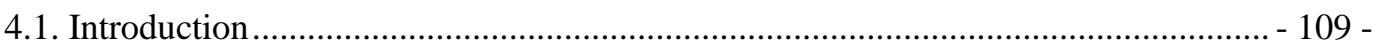

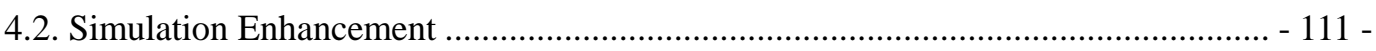

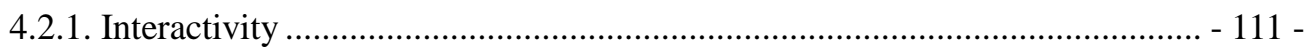

4.2.2. Collaboration via network ...................................................................... - 113 -

4.2.3. After Action Review - recording and reviewing ........................................... - 115 -

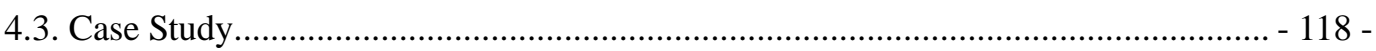

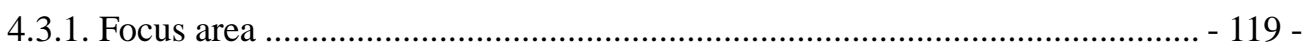

4.3.2. Static object modelling in SIEVE-VTS ...................................................... 120 -

4.3.3. Fully-hypothetic simulation ..................................................................... - 121 -

4.3.4. Semi-hypothetic simulation ................................................................... 126 -

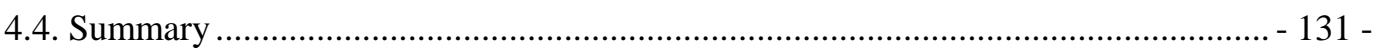

Chapter 5 System Test and Evaluation ............................................... 133 -

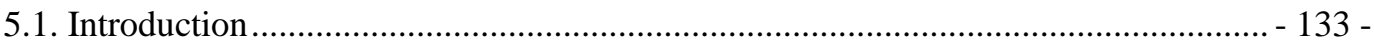

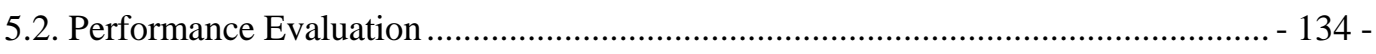

5.2.1. Rendering performance and scene complexity ............................................ - 134 -

5.2.2. Roundtrip time and real-time data updating frequency .................................. - 136 -

5.3. Functional Test and Feedback ........................................................................ - 138 -

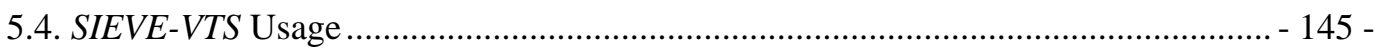

5.4.1. Emergency monitoring and response using SIEVE-VTS ................................ - 145 -

5.4.2. Emergency training using SIEVE-VTS ..................................................... 147 -

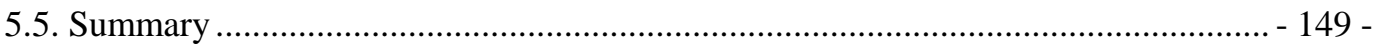

Chapter 6 Conclusions and Further Outlook ..................................... 153 -

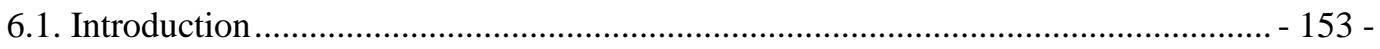

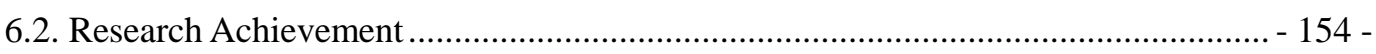

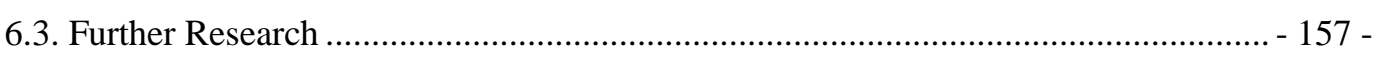

6.3.1. Collecting high quality real-time data ............................................................ 157 -

6.3.2. Improvement on scenario simulation .......................................................... 159 -

6.3.3. Distributed interactive simulation ................................................................. - 161 -

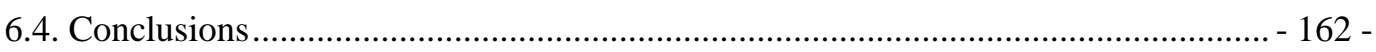

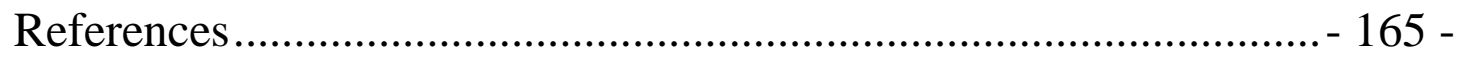

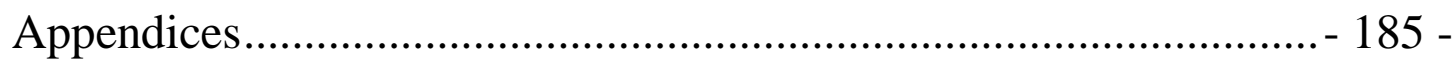




\section{List of Figures}

Figure 1.1: Emergency management continuum (Public Safety Canada, 2010, p. 4) ...............2

Figure 1.2: Emergency management using geo-spatial information: toxic spill in

Williamstown, Victoria (SpatialVision, 2010) ........................................................

Figure 1.3: Emergency management using CVE (VSTEP, 2010) ........................................6

Figure 2.1: Virtual military training using VBS2 (Bohemia Interactive Simulations, 2010)- 21

Figure 2.2: 3D visualisation in ArcScene (Land Trust GIS, 2010).................................... - 27 -

Figure 2.3: Spatial information visualisation in Vega Prime (PRESAGIS, 2010a) ........... - 28 -

Figure 2.4: NASA's WORLD WIND (NASA, 2010)....................................................... 30 -

Figure 2.5: Using Google Earth and CVE to visualise weather information

(GoogleEarthHacks, 2010)............................................................................ 32 -

Figure 2.6: Connecting GIS and visualisation using SIEVE (Stock, Bishop, Sharma, \& Chen,

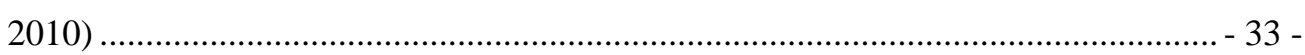

Figure 2.7: SIEVE Builder interface (O'Connor, 2007) ................................................ - 35 -

Figure 2.8: Architecture of mashup technology (Oracle, 2010a) ...................................... - 41 -

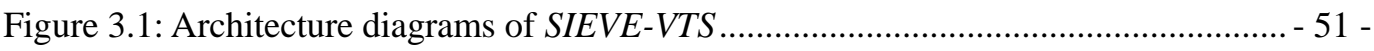

Figure 3.2: Flow-chart of RTDCollector working procedures .......................................... - 54 -

Figure 3.3: RTDCollector Graphic User Interface (GUI) ................................................. - 55 -

Figure 3.4: Snapshot of weather information from BOM ………................................ - 57 -

Figure 3.5: HTTP header of a request ................................................................. - 57 -

Figure 3.6: Parsing real-time weather condition ........................................................... 58 -

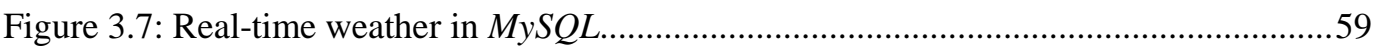

Figure 3.8: Oceanographic information in online service: tide time and heights............... - 60 -

Figure 3.9: Oceanographic information in online service: swell height and direction....... - 60 -

Figure 3.10: Parsing tide information into DOM ........................................................... 61 -

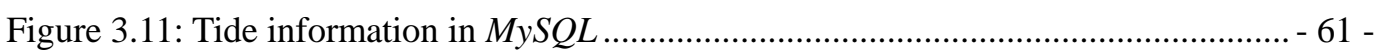

Figure 3.12: Mashup technique architecture (Gladstone USA, 2010) ................................ - 62 -

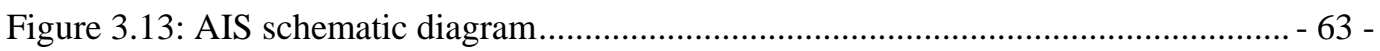

Figure 3.14: Online AIS data (University of the Aegean, 2010)...................................... - 64 -

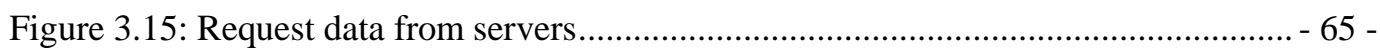

Figure 3.16: AIS information in an XML file ................................................................. 65 -

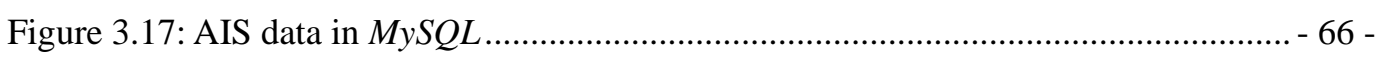

Figure 3.18: Weather in RSS from Yahoo! Weather..................................................... - 67 -

Figure 3.19: Auxiliary weather information in $M y S Q L$............................................... 67 -

Figure 3.20: CFA incident summary website (Country Fire Authority, 2010) .................... - 68 -

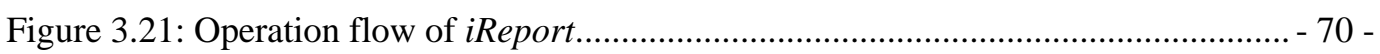

Figure 3.22: iReport user interface...................................................................... - 71 -

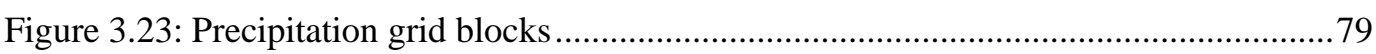

Figure 3.24: Different types and amounts of precipitation ............................................... - 80 -

Figure 3.25: Simulation of daylight in SIEVE-VTS..................................................... - 82 -

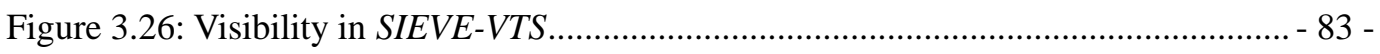

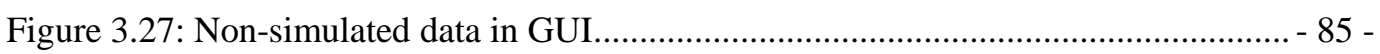


Figure 3.28: Tide simulation in SIEVE-VTS

Figure 3.29: Class diagram of vessel in SIEVE-VTS ................................................. 92 -

Figure 3.30: Type of incidents ............................................................................ - 100 -

Figure 3.31: Scale of incidents ........................................................................... 100 -

Figure 3.32: Delaunay triangulation and corresponding Thiessen polygon network for a set of scattered points (Aquaveo, LLC., 2010) ......................................................... - 104 -

Figure 4.1: Interactivity examples...................................................................... 113 -

Figure 4.2: GUI of network collaboration.............................................................. - 115 -

Figure 4.3: After Action Review in SIEVE-VTS: log in panel ..................................... - 117 -

Figure 4.4: After Action Review in SIEVE-VTS: timeline control............................... - 117 -

Figure 4.5: Research area - Sydney Harbour .............................................................. - 119 -

Figure 4.6: Static object conversion in SIEVE-VTS ................................................ - 121 -

Figure 4.7: User interface of SIEVE-TGEA-Exporter ............................................ - 121 -

Figure 4.8: Events marking for fully-hypothetic simulation........................................ - 124 -

Figure 4.9: Detailed design .................................................................................. 126 -

Figure 4.10: Time line design for semi-hypothetic simulation ............................... - 128 -

Figure 5.1: FPS and scene complexity ................................................................. 135 -

Figure 5.2: Roundtrip time and updating frequency .............................................. - 137 -

Figure 5.3: Comparison of marine traffic.................................................................. - 139 -

Figure 5.4: Comparison of incident location and status.......................................... - 139 -

Figure 5.5: Positioning in SIEVE-VTS .............................................................. - 140 -

Figure 5.6: Tools for emergency management: A) COSMOS; B) UnrealTriage; C)

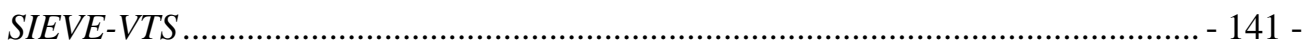

Figure 5.7: Comparison of single and multiple users ............................................. - 142 -

Figure 5.8: User's estimation of visibility ............................................................ 143 -

Figure 5.9: User's estimation of water level ........................................................ 144 -

Figure 5.10: User's estimation of ship speed .......................................................... - 144 -

Figure 6.1: Sensor Web Enablement (Franklin, 2007) .................................................. - 159 -

Figure 6.2: HLA for SIEVE-VTS (adapted from Calytrix Technology, 2010) ................. - 162 - 


\section{List of Tables}

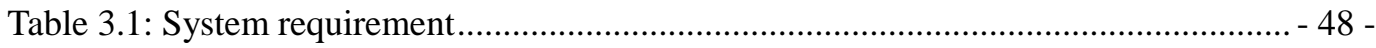

Table 3.2: Comparison of TGEA and other software ……............................................. 53 -

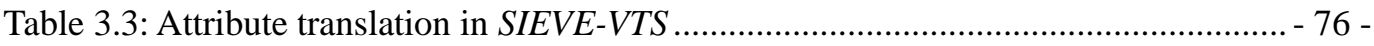

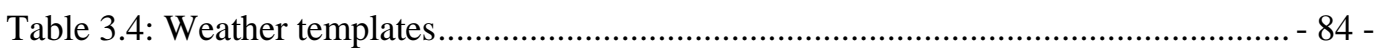

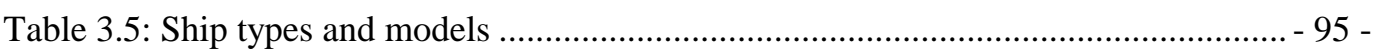

Table 4.1: Interactivity of virtual objects in SIEVE-VTS............................................ - 112 -

Table 4.2: SIEVE-VTS enhancement for scenario simulation .......................................... - 118 -

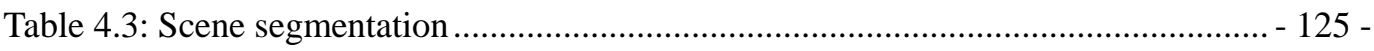

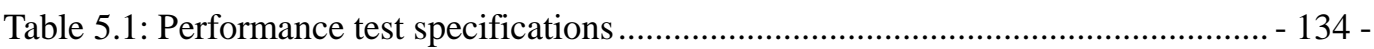

Table 5.2: Object complexity ……………………….............................................. - 136 - 


\section{Acronyms}

AAR

AIS

API

$\mathrm{BOM}$

CFA

CRC-SI

CVE

DEM

DSTO

GIS

ICT

MMSI

SIEVE-VTS

TGEA

XML
After Action Review

Automatic Identification System

Application Programming Interface

Bureau of Meteorology

Country Fire Authority

Cooperative Research Centre for Spatial Information

Collaborative Virtual Environment

Digital Elevation Model

Defence Science and Technology Organisation

Geographic Information System

Information and Communication Technology

Maritime Mobile Service Identity

Spatial Information Exploration and Visualisation

Environment - Virtual Training System

Torque Game Engine Advanced

Extensible Markup Language 


\section{Chapter 1 Introduction}

\subsection{Overview}

In present-day society, the threat of disaster is becoming increasingly worrisome. A great many disasters derived from one cause or another have been witnessed. Representative examples of natural disasters are: the 2008 Sichuan earthquake in China, in which about 70,000 were confirmed dead and thousands of people were listed as missing or injured (The New York Times, 2009); and the February 2009 Victorian Bushfires, resulting in the highest loss of life in a bushfire in Australia (Huxley, 2009). Man-made disasters include: the September 11 attacks in the U.S., killing more than 2,000 people and causing economic losses worth billions of dollars (Glazier, 2008); the nuclear incident at the Chernobyl Nuclear Power Plant in Ukraine on 26 April 1986, which was estimated to have cost 4,000 lives as well as long-term nuclear contamination in the vicinity (Black, 2011). Such disasters usually occur rapidly and are beyond people's control, and they pose severe threats to human lives and properties, and lead to considerable losses.

The frequent occurrence of disasters has raised people's consciousness and increased their efforts to prevent, manage, and resolve the resulting issues. Although disasters are sometimes thought to be unavoidable, it is possible to establish a set of methodologies and strategies to prevent or alleviate the consequences, and reduce the losses to a minimum level. Therefore, disaster or emergency management has been seen to be of great importance, as we seek to deal with these issues. Disaster or emergency management is defined as a continuous process by which individuals, groups, and communities manage disasters in order to avoid or meliorate the impact of major events. The process of emergency management involves four phases: mitigation, preparedness, response, and recovery (Haddow \& Bullock, 2003). Each of the phases includes various activities, focusing on solving the issues within different periods of time. The phases are linked together to form a circle of emergency 
management (Figure 1.1).

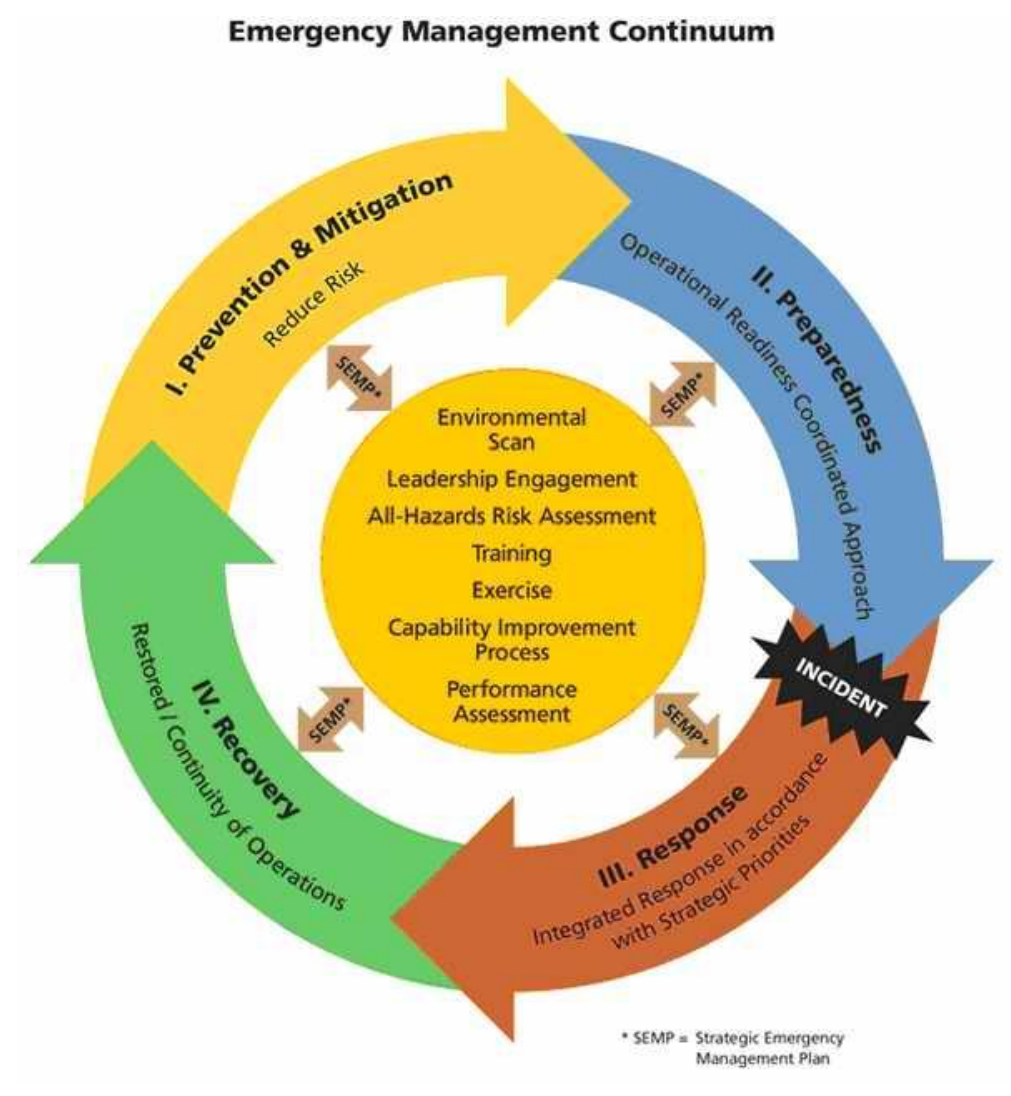

Figure 1.1: Emergency management continuum (Public Safety Canada, 2010, p. 4)

In emergency management, information and communication technology (ICT) plays an important part in enhancing emergency managers' and decision makers' capabilities to deal with emergencies. The availability and quality of information are central to effective emergency management. ICT also enables emergency management organisations, fire service personnel, first responders, homeland security and the general public to conduct necessary activities in any phase of an emergency in a faster way. Information critical to emergency management includes: spatial information of hazard areas; weather and climate conditions; location, status, scale of incidents happening; estimated losses and casualties; evacuation route and responders information (Chen, Sharman, Chakravarti, Rao, \& Upadhyaya, 2008). The importance of effective and efficient emergency management implies the use of recently developed tools and technologies to access information. 
Novel technologies are being applied to handle various kinds of information. These include: Internet, wireless technology, Geographical Information System (GIS), direct and remote sensing, emergency management decision and support systems, hazard analysis, modelling and warning systems, and wireless sensor networks (Rao, Eisenberg, \& Schmitt, 2007). These emerging tools and technologies can enhance communication capabilities, and make the usage of information more comprehensive, ready-to-go, user-friendly and affordable.

An essential property of information is its spatial attribute, which may refer to the location of an incident, real-time position of a vehicle or evacuation routes in the context of emergency management. Such information is often categorised as geospatial information and can be manipulated by GIS. GIS technology is important for emergency management because it enhances management capability by digitally capturing, storing, analysing and manipulating spatial information, and it takes effect in all phases of emergency management (Figure 1.2). For example, in the emergency prevention and mitigation phase, decision makers can evaluate the consequences of potential earthquake incidents based on spatial information, such as land use, terrain slope, soil condition, and other geologic data (Alparslan et al., 2008). In addition, spatial information such as fire station location and evacuation routes can be collected and displayed by GIS to provide assistance for emergency preparedness (Cole et al., 2005). During the emergency response phase, Automatic Vehicle Locating (AVL) can be integrated to track the position of emergency units in real time (Baldus \& Kim, 2005). A GIS can also work with the aid of GPS to locate damaged facilities, determine the amount and type of damage, and begin to set up priorities for actions in emergency recovery (Johnson, 2000). 


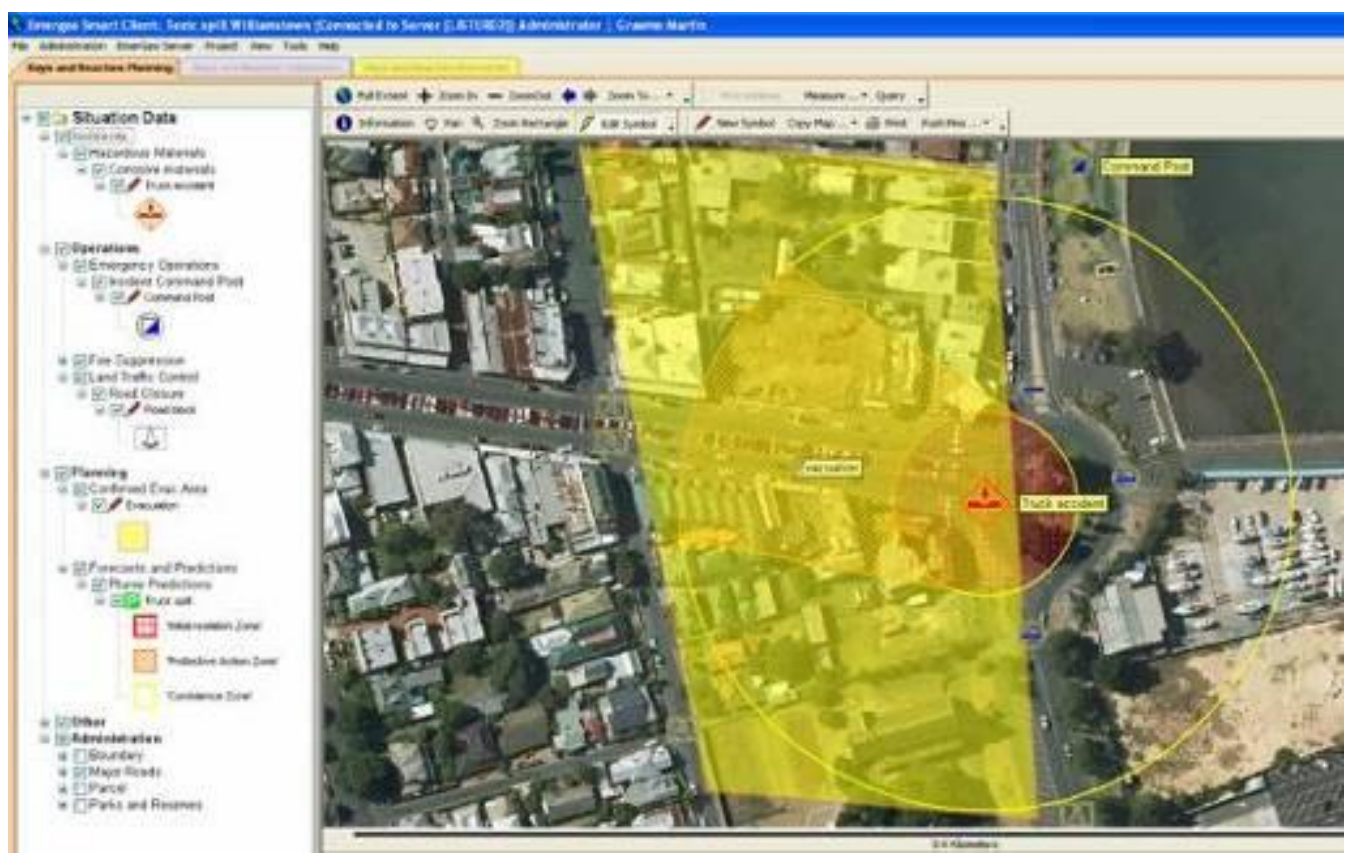

Figure 1.2: Emergency management using geo-spatial information: toxic spill in Williamstown, Victoria (SpatialVision, 2010)

Another property of information is its time attribute. In emergency management, time has been highly emphasised due to its limited and critical nature during an emergency. It is particularly important to deliver up-to-date data as quickly as possible, as timely provision of information can greatly help in the decision-making process, save lives and aid citizens. For example, real-time weather conditions are critical for emergency responders' decision-making when evacuating civilians in a fire accident, since the meteorological processes may change and influence the fire condition. Traffic condition is also important for emergency evacuation operations as it provides basic information for emergency responders making evacuation plans. Furthermore, real-time water level in oceanic or fluvial areas can be monitored to determine the extent of local flooding. Webcams can also capture extra information from on-site locations to deliver incident information to emergency managers. Related research is developing fast, representative examples include using GPS, PDA, smart phones and distributed sensors in targeted areas to monitor the latest conditions of incidents.

When information is delivered to end users, it needs to be expressed and represented in an appropriate way. While some information is delivered in text, table, image, 
animation or other multimedia formats, spatial information has traditionally been expressed through maps. With the development of ICT, people have more opportunities to explore spatial information in new formats, which is transitioning from traditionally used abstract maps to representations which look more like the real world, allowing people to understand what is being presented to them more easily. 3D representations of spatial information have been investigated to assist communication of concepts, learning of tasks and decision-making (Hofschreuder, 2004), and have been applied in a range of fields such as visualising and analysing geological hazards (Carrara, Guzzetti, Cardinali, \& Reichenbach, 1999), land information management and urban planning (Ross, Bolling, Döllner, \& Kleinschmit, 2009), and military command and control (Pettersson, Spak, \& Seipel, 2004).

Emergency management can achieve greater benefits from 3D spatial information compared with 2D spatial data. 3D spatial information offers more sophisticated visualisation and data analysis, more interactive experience within the environment, more precise orientation and positioning, and advances in underground and indoor analysis, which are typically unachievable using 2D information (Zlatanova, Fabbri, \& $\mathrm{Li}, 2005)$. Research in this field is occurring in many domains and includes building evacuation models in urban areas using 3D spatial information (Kwan \& Lee, 2005; Choi \& Lee, 2008), and disaster simulation and analysis using 3D visualisation (Kolbe, Gröger, \& Plümer, 2008; Lee \& Zlatanova, 2008).

Collaborative Virtual Environment (CVE) is another emerging concept, which fully utilises and advances 3D visualisation technology (Figure 1.3). CVE is used to support the collaboration and interaction of a large number of participants who may be geographically spread over large distances (Pečiva, 2007). Typical examples are distributed simulations and 3D multiplayer online games. Firstly, CVE utilises Virtual Reality (VR) technology, providing users with computer-generated environment that cannot only simulate entities present in the real world but also objects in imaginary worlds. Each user is represented as an avatar with abilities to immerse within, 
navigate through, and manipulate the CVE. Secondly, CVE enables multiple users from different geographic locations to join to one common space and interact with the same virtual environment simultaneously, therefore CVE shows advantages in enabling communication and collaborations between users. Further potential benefits of CVE include: improvement on planning processes, products, and tools, enhancement of training, education and professional development of emergency response planners, establishment of better foundations for collaborative methods, creation of lower cost simulations, as well as supporting task-directed distributed collaboration (Monahan, Mustanski, \& Neuner, 2009; Wickler, Potter, Tate, \& Hansberger, 2011).

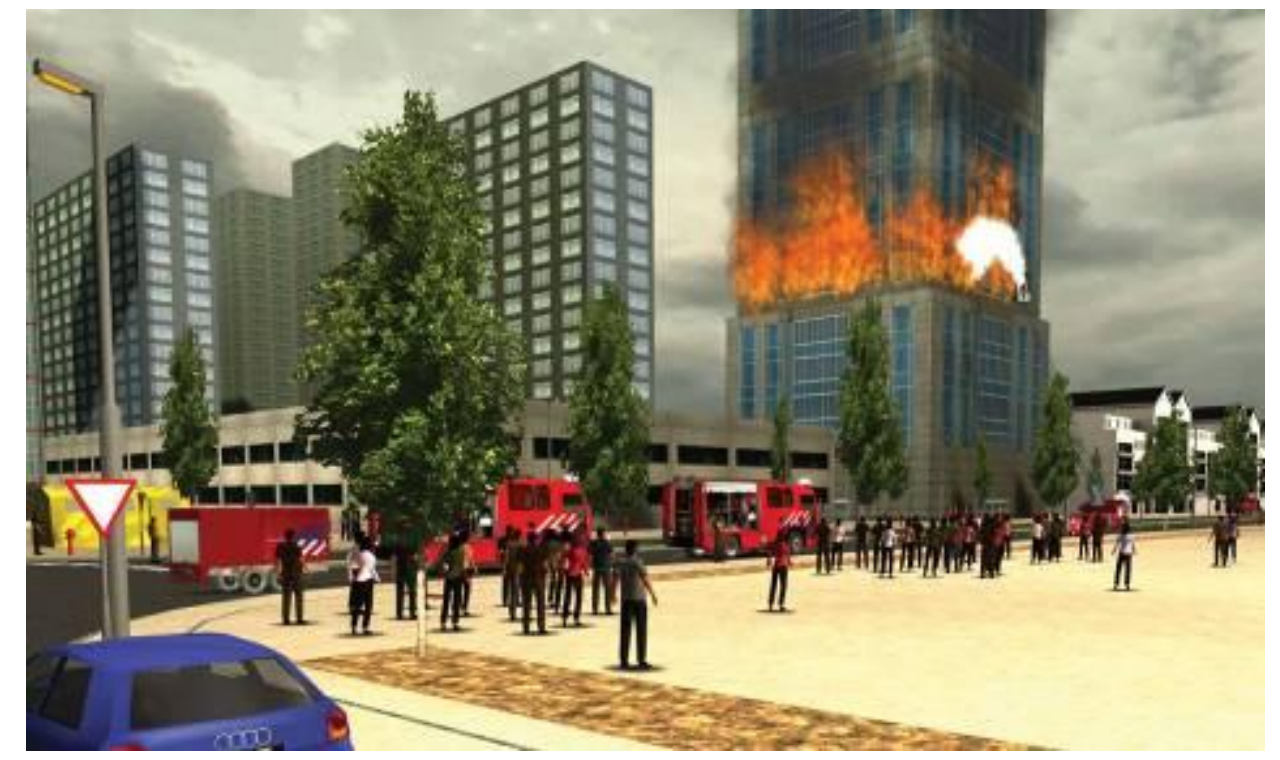

Figure 1.3: Emergency management using CVE (VSTEP, 2010)

The proposed research focuses on the integration and visualisation of dynamic spatial information and CVE, and puts it into practice for emergency management. Dynamic data not only contains information about location, but also includes temporal attributes, for location and other attributes may change with time. If such data is provided within an area of interest using CVE instead of 2D maps, users can explore and utilise the spatial information in more diverse ways. In such an environment, users cannot only view static objects such as terrains, buildings, vegetation, but also explore dynamic changes such as weather, traffic and emergency incidents. 
Furthermore, if multiple users can also share information and communicate with each other through the Internet, collaborative teams can be formed and better decision support will be achieved. Using such a system, emergency responders can obtain real-time incident information to initiate an emergency response. Emergency management agencies can also use the system for emergency training.

This thesis discusses the development of a system that realises some of these possibilities, addresses limitations encountered and outlines future directions. Specifically, it describes a system which collects real-time spatial information from multiple sources, and visualises it in a CVE.

\subsection{Research Problem}

In emergency management, the applications of 3D and 4D visualisation of spatial information are increasing, CVE and related technologies are being widely promoted by researchers in this field (Wright \& Madey, 2008; Zarraonandia, Vargas, Díaz, \& Aedo, 2009; Chen, Rebolledo-Mendez, Liarokapis, De Freitas, \& Parker, 2008; Van Ruijven, 2011). Nonetheless, some issues exist in currently used technologies and software: various types of spatial information cannot be easily integrated and visualised in CVE; problems like low authenticity of the emergency simulation and delay of data delivery often result in failure to fulfil the user's requirements of sharing information in a time-limited condition or responding quickly to emergency incidents based on known information (Miao \& Haake, 2001; Chen \& Kalogeraki, 2005). Therefore, such CVE-based applications do not provide enough aid to emergency managers' decision making or promote adequate collaborative communication.

The authenticity of emergency simulation in CVE still needs improvement. Some emergency management CVE have been elaborately designed to enhance their authenticity, for example, by improving the graphic quality, the validity of scenarios, and the human-computer interaction (Blasband, Bleak, \& Schultz, 2004; Walton, Patton, \& Parsons, 2001; Wu, Ott, \& Miller, 2009). However, a very important factor 
which has not been sufficiently addressed is whether the description of emergency events in the virtual environment corresponds to those occurring in the real world (Mantovani, Gamberini, Martinelli, \& Varotto, 2001; Wyld, 2010). In other words, although virtual environments are composed of high quality static models such as terrains, buildings, and intelligent non-player characters that perform like real human beings, the dynamic variations are not accurately simulated according to the real world situation. This is particularly the case when users operate in a large-scale and complex emergency situation, such as responding to a wild fire or earthquake. When the information from the real world is limited, the reality represented in the virtual emergency scenarios is far from real, which often limits the users' potential to properly respond to the hazard at hand.

Delivery of up-to-date information cannot be guaranteed in currently available CVE. When integrating information into platforms that can be utilised for emergency management, it is still challenging to deliver such information in a satisfactory time. There are several steps involved such as collecting, analysing, storing and visualising data before it is finally employed by the end users. Such processing procedures cause unavoidable delays. If the data is no longer valid, it may impede fast response or decision making by users. This is especially important when delivering information such as real-time traffic data or instant incident status in an emergency response situation.

The problems with usability of CVE include: the lack of a standard for creating and utilising dynamic simulation that is broadly accepted by industry and academy; creation of virtual environments and scenarios in the CVE is time consuming, for there is no automatic way to build dynamic scenarios from the original data source; the functions of multiple user collaboration need further enhancement, such as collaborative sharing and analysis of spatial information, collaboration during and after the simulation, as well as more efficient networking performance.

Addressing these issues and delivering existing data fast and automatically in CVE 
will aid the visualisation of real-time spatial information. 3D visualisation of spatial information in a multi-user system will further aid communication between users and enhance community engagement and decision making.

\subsection{Research Objectives}

The overall aim of this research is to advance techniques for simulating real world events within CVE. In particular, the work seeks to facilitate better decision making processes in the context of emergency management, through offering additional insights to users in a complex virtual environment.

As such, the research objectives are to:

- Investigate and design new techniques to integrate real-time data from multiple sources in a cost-efficient way.

- Model and visually simulate real-time data (such as weather, traffic condition, incident location) in an emergency management situation.

- Develop a virtual simulation platform and an updated user interface for emergency management in CVE.

- Test and evaluate the functions, performance and usability of the developed system through a client driven case study.

\subsection{Thesis Structure}

The remainder of this thesis consists of 5 chapters. Chapter 2 provides the research background, reviews current technologies and tools which are utilised in the field of emergency management, and highlights the strengths and weaknesses of these technologies. It discusses related research including CVE that provides advantages in visualising, modelling and simulating incident scenarios for emergency management, 
real-time data integration technology that enables users to collect and store real-time data immediately after an incident happens, spatial and temporal models that build the relationship between the spatial and temporal attribute of data. This chapter establishes the foundations for basic understanding of the existing literature and lays out a guideline for the following research.

Chapter 3 incorporates the techniques outlined in Chapter 2 and describes the design and implementation of a prototype system. The design is based on users' potential needs, current technology development and requirements analysed from the literature. The functionality of integrating, translating and visualising maritime-related real-time information is detailed. The components of the system are outlined and include a real-time data collector, a translator, a real-time data simulator as well as an event-based geo-database. Each component is described in detail with its functionality, related algorithms and implementation. The chapter also describes an interpolation method which is applied to address the problem of displaying information in an area where no real-time data is available. Representative data examples are selected and demonstrated in order to test the system capability.

Chapter 4 explores the system's potential to simulate emergency scenarios which are used for exploring historical events or observing current situations in emergency training and monitoring. To achieve this objective, the functions developed in Chapter 3 are used for connecting a real world situation with virtual simulations. Additional enhancements of capabilities are also introduced, including virtual objects interactivity, game Artificial Intelligence (AI), collaboration through the network, and After Action Review. Based on these, the virtual simulation can be carried out with the collaborations of users under certain regulations. A case study is designed for incident scenario simulation in the Sydney Harbour area, and the applications of simulations using traditional and new methods are discussed and compared.

Chapter 5 summarises the performance and functional testing of the system. Performance tests focus on the rendering capability and network performance. 
Functional testing is conducted with a defence organisation in Sydney that has access to the system. Their feedback is discussed and analysed with reference to the functional requirements. Potential usage of this system is also discussed based on users' view and system requirement.

Chapter 6 discusses the findings of the research, explores the results relative to the research objectives, suggests potential areas for future work and concludes the thesis. 



\section{Chapter 2 Literature Review}

\subsection{Introduction}

Emergency management consists of 4 phases: mitigation, preparedness, response, and recovery. Mitigation is working towards preventing hazards from developing into catastrophes, or lessening the effects of an emergency when it happens. Preparedness is a continuous process of planning, organising, equipping, evaluation and improvement to ensure effective coordination and enhancement of capabilities to prevent, protect against, respond to, recover from, and mitigate the impact of natural and man-made disasters. Response is the action of emergency services and emergency responders in the affected area. Recovery is to restore the affected area to its previous status after the emergency occurs (Haddow \& Bullock, 2003). Among these phases, emergency preparedness and response are critical when dealing with an emergency in order to save lives and reduce losses.

Emergency monitoring involves monitoring situations and collecting data in certain areas which are possible locations for incidents. The monitored data provides timely decision support for end users, including emergency managers, planners, first responders, voluntary agency coordinators, and other emergency management professionals. Emergency monitoring involves technologies such as remote sensing, geospatial information, mobile devices, distributed sensors, and integrated monitoring and responding systems. These technologies are widely utilised in various fields of emergency management, and have shown their effective performance (Ramsey et al., 2004; Nasui, Oana, \& Sgarciu, 2010; Madey, Szabó, \& Barabási, 2006; Zhu, Tang, \& Du, 2010; Upadhyay et al., 2009).

Emergency training is an important sub-phase of emergency management, connecting the phases of preparedness and response. It is a practice activity that involves trainees in a simulated situation, where they are asked to respond to hypothetical emergencies 
that could potentially take place in the real world. Advanced technologies are now regularly used in this activity and have greatly improved the quality of training (ETC Simulation, 2010; Bohemia Interactive Simulations, 2010; Manganas et al., 2004). For example, interactive simulation using 3D virtual environment (VE) was shown to be effective in helping trainees understand the surrounding geographical information, get familiar with facilities, and test emergency response plans (Jain \& McLean, 2003).

One technical feature of emergency management is the usage of spatial information. The storage and transfer of spatial information are now commonplace, the spatial analysis tools have become increasingly sophisticated, and geo-visualisation tools are getting more popular and advanced. The development in spatial information technology has enabled its application in emergency management. Well-designed software and integration of technologies will provide ready access to spatial information outputs and intelligent emergency management.

Another technical feature of emergency management is real-time data access by decision makers and emergency managers. The types of real-time data are diverse and include weather information, incident location and status, traffic conditions in the emergency-affected area. Technologies and tools for collecting, processing, and visualising real-time data in emergency management need further review in this chapter.

This research focuses on advancing emergency management by integrating technologies such as the 3D Collaborative Virtual Environments (CVE), real-time data collection and visualisation, and spatial information management and analysis techniques. The advantage of such integration is that people with limited knowledge of GIS or 3D computer modelling, can access sophisticated simulation scenarios. In real emergency situations, such systems have the potential to support appropriate responses to complex and threatening conditions, where intuitive understanding of emergency information is critical. This chapter reviews the trend of each technology, identifies the merits and deficiency in related work, and establishes foundation for this 
research.

\subsection{Emergency Monitoring for Response}

\subsubsection{Emergency monitoring introduction}

Emergency monitoring involves obtaining information about the emergency incident. The primary purpose is to provide timely information to assist decision making on which protective actions can be confirmed or revised. It plays a significant part in all stages of emergency management. In the preparedness phase, environmental information of a certain location is monitored on a daily basis, including weather condition, air and water quality. These data provide useful background information for the emergency managers and first responders. In emergency response, the latest information about incident status and the affected population must be accessed rapidly. In the recovery phase, monitored data is used for damage assessment, aiming to support rehabilitation and ongoing redevelopment activities. For example, in the nuclear incident of Fukushima in Japan (Negishi, 2011), certain organisations monitored the environmental radiation level and weather condition in the contaminated area. The monitored data was then used by decision makers to take protective actions, evacuate victims in the emergency, and assign rescue teams to the affected area.

Generally speaking, an emergency monitoring strategy starts from the prior identification of potential hazardous situations and extends to environmental sensing performed during the late phases of an incident. It combines the use of fixed monitoring stations with mobile measurements. The monitored results can provide accurate and timely information on the level and degree of a hazard, as well as its extent and duration. Emergency monitoring generates useful datasets for public security managers, emergency response team, traffic control personnel and civilians (Wisconsin Department of Transportation, 2008; Delaware Department of Transportation, 2006; U.S. Department of Transportation, 1998). 


\subsubsection{Emergency monitoring techniques}

Recent technological advances related to emergency monitoring include the improvement of hardware and software, as well as enhanced methodologies for collecting, analysing and utilising monitored data in emergency situations.

One trend is the use of remote sensing technologies to monitor emergency incidents in near real time mode (Ramsey et al., 2004; Shao, Gong, Wang, Zhang, \&Tian, 2009; Hedge, Shalini, Nayak, \& Rajawat, 2008). Research directions include multi-source data integration, information extraction and analysis, and high-resolution data acquisition. Using remote sensing to monitor incidents can provide rapid response solutions for detecting both natural and anthropogenic disasters, and it has a particular advantage in monitoring large scale hazards such as landslides, earthquakes, floods, and water pollution. It also offers high-quality information to the public, facilitates collaborations of emergency services, assists timely decision-making, and potentially prevents or mitigates the damage.

Another trend is the use of mobile devices or sensors to monitor emergency situations. Users could obtain real-time information about any monitored vehicles, such as location and routes taken, they could also retrieve information from the sensors, including radiation, smoke or gas levels. Every entity communicated with each other over a variety of wireless networks. The time involved in searching for and responding to emergency situations was significantly reduced (Nasui et al., 2010). Wireless sensors integrated in indoor environments provided a cost-efficient way to detect emergency events, identify hazardous regions, and uncover secure evacuation routes (Pan, Tsai, \& Tseng, 2006). Sensors embedded in garments could monitor the health state of users, surrounding environmental factors, as well as incident location and status. Date was transmitted in real time, providing timely information for emergency operators and first responders (Magenes, Curone, Caldani, $\&$ Secco, 2010). 
An integrated monitoring and response system is another popular research topic. Madey et al. (2006) developed a Wireless Phone Based Emergency Response (WIPER) system which can collect real-time data from numerous mobile phone users, discover uncommon human aggregation, possible emergencies and traffic congestions. The overall system was composed of a data source and measurement module, a historical data module, a detection and alert module, a simulation and prediction module, and a decision support module. WIPER could monitor, forecast and propose responses to a variety of emergency situations, and it was also able to provide tools for estimating possible courses of action in dealing with emergency situations, and generate datasets of potential value to the end users (Schoenharl, Madey, Szabó, \& Barabási, 2006; Madey et al., 2006). Tseng, Shu, Horng, Kuan, and Hsu (2007) designed a Central Monitoring System $(C M S)$ to operate between the emergency response centre and many buildings, with the aim of integrating all types of safety apparatus critical to human life and property. It allowed efficient conveying of data such as the presence of toxic materials, the affected area, nearby buildings, waterways, and traffic conditions. In this way, the system could increase the overall efficiency through saving resources, manpower, and the associated operational costs.

Emergency monitoring plays an important role in many areas. One example is environmental emergency monitoring, including accidental water pollution, oil spill, and radiation (Zhu et al., 2010; Lahtinen, 2004; Kim, Moon, Hwang, \& Kim, 2010). It provided rapid and full technical support to security monitoring, and offered timely information for the corresponding monitoring department. Emergency managers could make decisions based on incident location, time and development trend. Emergency monitoring techniques can also be applied in large scale geological hazards, such as earthquakes, landslides and flood (Shao et al., 2009; Surendranath, Ghosh, Ghoshal, \& Rajendran, 2008; Bonn \& Dixon, 2005). Information about the start, trend, and results of the incidents can be recorded and submitted to the emergency management agencies. Another case of emergency monitoring is controlling fire accident, which is emphasised as a common type of emergency 
incident. Indeed, researchers have integrated wireless sensors, grid-enabled High Performance Computing (HPC), and artificial intelligence tools to build up fire emergency response systems (Upadhyay et al., 2009; Zeng et al., 2011). Monitoring can greatly benefit fire emergency response, as it provides first responders with useful information and enables them to make correct and conversant judgments while dealing with the fire.

\subsection{Emergency Training and Implementation Tools}

\subsubsection{Emergency training introduction}

Emergency preparedness is an important phase in the emergency management cycle. It includes various activities such as planning, training, equipping, and evaluation, aiming to establish effective collaboration and capability to protect against, respond to and recover from natural or man-made disasters (U.S. Department of Homeland Security, 2007). Among all the activities, emergency training is considered to be one of the key tasks.

An emergency training is a practice activity that gathers trainees in a simulated condition and instructs them to execute a series of tasks that would potentially occur in a real emergency event. Emergency training enables participants to become familiar with the geographic environment, to test equipment functionality, to clarify their roles and responsibilities, and to improve individual performance and team collaboration. It can also reveal errors in planning, discover shortage in facilities and avoid mistakes and losses in real emergency situations (Federal Emergency Management Agency, 2008). Emergency exercises are usually divided into two categories: discussion-based exercises and operation-based exercises.

Discussion-based exercise involves discussing existing or new plans, policies, procedures and issues, in the form of seminars, workshops, tabletops, and so on. An information session is one way of delivering a discussion-based exercise 
(Transportation Research Board of the National Academies, 2006). It conveys information to a varied or specific audience, and is usually held in the form of seminars or workshops. Tabletop is another form of discussion-based exercise. It designs a casual and relaxed environment for participants to discuss different issues regarding hypothetical situations. The advantages of hosting a discussion-based exercise are: it allows the group to gain a good understanding of agency roles, actions and limitations through interactive discussion; it is relatively easy to organise; it is flexible in different forms and is effective in generating results. However, its disadvantage is that the exercise is not realistic enough to represent the real situation, and the interaction between attendees may be inadequate so that the effect of training is weakened.

Operation-based exercises are activities taken to respond to a simulated emergency in an area over a continuous period of time, with the purpose of testing facilities and equipment, and validating the plans, policies and procedures. Examples of operation-based exercises are drills and field exercises. A drill is a coordinated activity used to test specific functions in a simulated emergency situation. It is commonly used to train personnel in the use of new facilities, to develop or test new procedures or policies, and to exercise and maintain current skills (Virginia Department of Health, 2011). Field exercise is another practice activity that comprehensively assesses an emergency plan. It enables participants to organise trainings under real conditions and utilise their own knowledge in a time-intensive environment. Several agencies are involved in a field exercise, including public safety personnel, medical services, fire brigade, and government organisations (Federal Emergency Management Agency, 2008). Field exercise includes functional and full-scale exercises, which are different in scale but have similar contents and procedures. The strength of operation-based exercises is that the time intensive situation can provide trainees with a near real environment to assess the validity of plans and the effectiveness of operating procedures. However, the cost and complexity of field exercises mean that they require much effort to organise and 
cannot be undertaken as frequently as an organisation may wish.

\subsubsection{Virtual emergency training and related tools}

In discussion-based exercises, the traditional method of emergency training is to use text-based materials such as training manuals, textbooks and slide shows; some advanced techniques employ more visual presentations such as PowerPoint and video animations. With the rapidly evolving computer graphic technology, Virtual Reality (VR) has been utilised in emergency exercise to reduce cost and enhance the training effectiveness. In this section, several mainstream virtual training systems are investigated and introduced.

Virtual Battlespace $2(V B S 2)$ is an interactive, 3D, PC-based synthetic virtual environment (Bohemia Interactive Simulations, 2010). Being suitable for military training and experimentation, it provides developers with tools that could create, modify, and operate objects including terrains, buildings, vehicles, and artificial intelligence in the virtual environment. Trainees are taught doctrine, tactics, techniques, and procedures in offensive, defensive, and patrolling operation. Currently VBS2 is selected as a military training system by many organisations such as the Australian Defence Force, NATO members, the United Kingdom Ministry of Defence, and the United States Army (Bohemia Interactive Australia Pty Ltd, 2010). Some of its features that render it suitable for emergency training include: large area terrain editing, high dynamic range rendering, and the provision of a flexible collaborative training environment (Figure 2.1). At present, the primary users of $V B S 2$ are military personnel, and a fully-featured system is not open to the public at an affordable price. 


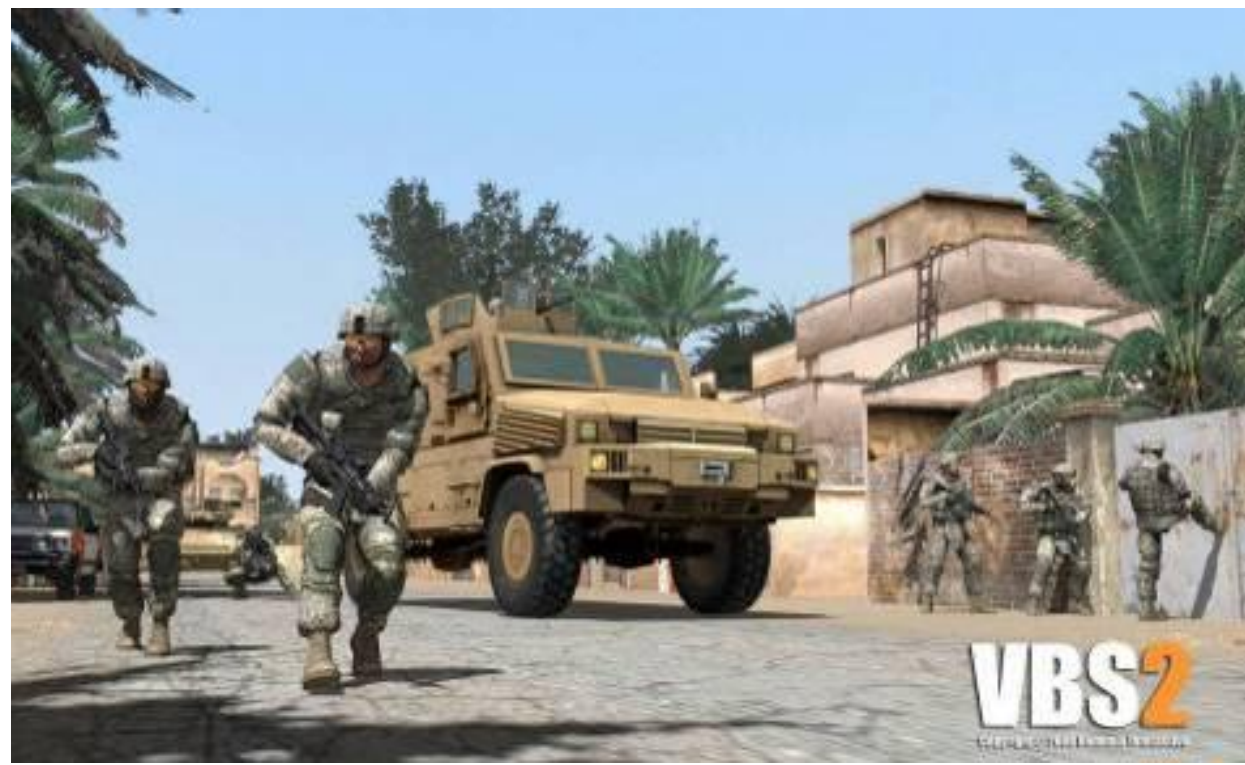

Figure 2.1: Virtual military training using VBS2 (Bohemia Interactive Simulations, 2010)

The Advanced Disaster Management Simulator $(A D M S)$ is a 3D VR training system consisting of different scenarios in various environments (ETC Simulation, 2010). The system is highlighted by a high-quality range of virtual objects such as incidents, vehicles, personnel and equipment. It supports the simulation of road traffic accident, hazardous material incidents, domestic fires and incidents in tunnels, railway and aircrafts. It enables participants to deal with emergency situations using virtual resources, and is ideal to be implemented in emergency disciplines.

The Entertainment Technology Centre and Carnegie Mellon University (2005) used video game technology to train first responders for chemical and hazardous material emergencies through a networked, multi-player simulation named "HAZMAT: Hotzone". The objective of this system is to enhance communication, observation, and critical decision making for emergency users.

The "Just VR" System (Manganas et al., 2004) is a hybrid system of hardware platform and software application, which includes networked PCs, high-level VR ancillary equipment, stereo projectors as well as virtual training software. The system not only performs interactive real time simulation through system kernel components 
but also drives external VR devices for users to control. In the simulation, trainees are equipped with a digital glove for virtual scene exploration and gesture communication, a head tracker for navigation, and wireless stereo glasses for observing the action. They can interact with the virtual environment by recognising the situation and responding in time. This system has been adopted for training personnel in medical emergencies and has been tested to be cost-efficient.

Second Life (Linden Research, Inc., 2010) is an online 3D virtual world. Currently, it is mainly used for entertainment, social networking and communication. It is ideal for simulating the real world due to its powerful ability to produce scenes, interactively create, remove, and modify objects and relevant attribute information, as well as support extensive multiplayer online collaboration. Second Life has been selected by several institutions and organisations for scientific research, education or training (Boulos \& Burden, 2007). It is a potential and promising option as a CVE for incident simulation and emergency training.

The available virtual training systems are diverse in functionalities and performance, but share similar usage patterns. The procedure of using such systems generally starts with building a virtual environment and designing a hypothetical emergency situation. Users then utilise the system to interact with, and respond to the emergency situation in the simulated exercise. Finally, the users evaluate and discuss the training effect following completion of the exercise.

Using VR technology to simulate an emergency situation for training has the following advantages (Kelly et al., 2002; Louka \& Balducelli, 2001):

1. It is practical to carry out high quality simulations using VR technology on desktop PCs because of promotion in computational technology, especially cheap graphics capabilities. It is sufficiently powerful to enable simulation of a large number of emergency situations, such as fire hazards, chemical incidents, traffic accidents, and so forth. 
2. Virtual training can provide users with timely information, and allow the customisation of training to unique local situations. Virtual environment offers a "virtual reality" that draws the learners perceptually and physically into it, and allows them to execute tasks and experiments similar to what they would do in the real world.

3. Training in VR allows users to experience performing otherwise dangerous tasks without harming themselves or impacting the environment. This is not easily achievable in a real training environment such as field exercise. Therefore a safer virtual environment can be used to simulate a real or planned event that may be too dangerous, complex, or expensive to train in.

4. A particular advantage over traditional training methods, such as books and video, is that using VR, trainees are active and can improve skills and cognitive ability through practice. Informal assessment suggests that the level of concentration and involvement required of the trainees in such an environment results in greater retention of acquired skills comparing with other forms of off-the-job training.

5. Compared with training in a real environment, VR training is flexible, convenient and inexpensive to organise. It has a high degree of deploy ability such that users can undertake virtual trainings on a $24 / 7$ basis, and it can be repeated as many times as the users desire.

6. VR training system can go a further step by integrating intelligent computer-based training functionality with a simulator. In addition to behavioural knowledge, the simulator can provide knowledge of correct or appropriate responses to situations, such that the system is able to guide the trainees, either to teach them a correct procedure or to assist them in making appropriate action.

Emergency training using VR can provide trainees with a near real environment to assess the validity of plans and the effectiveness of operating procedures in a time 
intensive situation. However, in practice, VR technology still cannot replace training in a real environment, where touch and feel are significant factors for learning. There remains considerable room for improvement in various aspects of virtual training technology, such as the authenticity of the virtual environment, artificial intelligence and more effective human-computer interactivity.

\subsection{Emergency Management with GIS}

In order to make emergency management more efficient in curtailing risk to human life and property, Geographic Information System (GIS) can be employed in all the stages of it. GIS plays a significant role of data acquisition, integration, storage, analysis and display in emergency management. The comprehensive datasets are beneficial for analysing emergency management options, directing tactics, and taking actions (Zerger \& Smith, 2003). Some applications of geospatial information in emergency management have been investigated and recent trends in these applications are now reviewed.

- 3D/4D spatial information in emergency management:

Traditional 2D GIS sometimes cannot adequately meet the requirements of emergency management in certain areas (Zlatanova et al, 2005). For example, non-expert users cannot fully perceive the situation in a 2D map; sophisticated spatial analysis and highly realistic data presentation are not well supported. Therefore, 3D/4D geo-information technology is urgently needed for emergency management. The advantages of multidimensional GIS outweigh those of 2D maps in many prominent aspects. Users cannot only orient themselves better and faster, but also acquire a better appreciation of the on-site situation. Lee (2007) proposed a new 3D data model for emergency response which represented an urban built environment in multi-levels, and integrated geometry, topology and CAD models. The implementation of such a model into an emergency management system benefited both field workers and decision makers. Beni, Mostafavi, and Pouliot (2007) created a dynamic 3D data 
structure supported by 3D Delaunay triangulation network, and provided an interactive topological mesh and numeral simulation. It was possible to do on-the-fly updating of topology with respect to any activity or change in the $3 \mathrm{D}$ environment.

- Multi-source spatial information integration:

Integration of multi-source data is another significant research topic. In emergency management, mass data must be collected, transferred and analysed in a short period of time. However, data heterogeneity is inevitable, which results in issues of data interoperability and information sharing. Since end users such as first responders and decision makers are not able to determine data usability, methods must be developed to ensure the seamless integration of heterogeneous data and appropriate data distribution in an emergency situation. To improve semantic interoperability and information sharing, the concept of ontology (Pundt, 2008; Xu \& Zlatanova, 2007; Kokla \& Kavouras, 2001), specified for emergency response and related approaches, was formalised. It was expected that such a method could help in identifying relevant data and in sharing of information in emergency situations. Furthermore, Schrenk and Pozarek (2007) introduced a method of combining cross-border-region spatial information from various sources for political and economic decision making, which is integrated into "Centrope Map" as the Spatial Data Infrastructure (SDI). The project involved integrating heterogeneous geospatial data and related attributes of the involved regions, overcoming the challenges of accessing data from different organisations, using multiple languages from different counties, and integrating multi-source data using various standards and tools. Zhao, Huang, Guo, Wen, and Zhong (2007) proposed a model with a three-layer structure solution based on collaboration of MapInfo, ArcGIS and Oracle Spatial. This model improved spatial data centralised storage and metadata interoperability. The above research demonstrated that data from multiple sources can be integrated into one common structure, and that the interoperability can be improved, which will greatly benefit the rapid collection and sharing of data in an emergency situation. 
- Web service for spatial information:

With the development of network technology, Web service has been employed to transfer and exchange geospatial information. Tanasescu et al. (2006) designed an emergency management application based on Semantic Web Service. It assisted the emergency officer in retrieving, processing, displaying and interacting with information relevant to the emergency. Security agencies such as Country Fire Authority (2010), Victoria and Chicago Police Department (2010) utilised geospatial Web services to display information about recent incidents, including incident location, type and status. Such information could help emergency responders analyse the current situation and also inform the public of security conditions.

\subsection{Spatial Information Visualisation in a 3D Environment}

The last chapter explained that visualising data in a 3D environment can significantly improve people's understanding, and can benefit decision makers, first responders, and emergency managers. For this reason, various kinds of technologies including software and hardware have been developed. They can be divided into several groups: 3D components of GIS software, non-GIS 3D visualisation tools, virtual globes and CVE.

\subsubsection{D functionalities in GIS software}

Traditional GIS software provides 3D functionality which can be used for visualising urban and rural environments. The environment is specified through 2D and 3D GIS layers: a digital elevation model, an image which is draped on the surface and polygon, polyline or point files which define the locations of surface objects such as buildings or trees. Specific functions are used to allocate appropriate 3D symbols to the locations.

A representative example of such software is ArcScene (Figure 2.2), a 3D 
visualisation application in ArcGIS Desktop (ESRI, 2010b). Users are enabled to visualise 3D data by extruding vector data from a surface to create lines, walls, and solids, or analyse 3D data such as hill shade and view shed. 3D icons of buildings or trees can also be allocated to point locations. Another example is NVIZ, a multi-dimensional visualisation suite for GRASS GIS (Open Source Geospatial Foundation, 2010). It allows users to render multiple layers in a 3D environment by using thematic colouring, adding vector files over the surfaces, and finally visualising GIS data either on the layers or as 3D point locations.

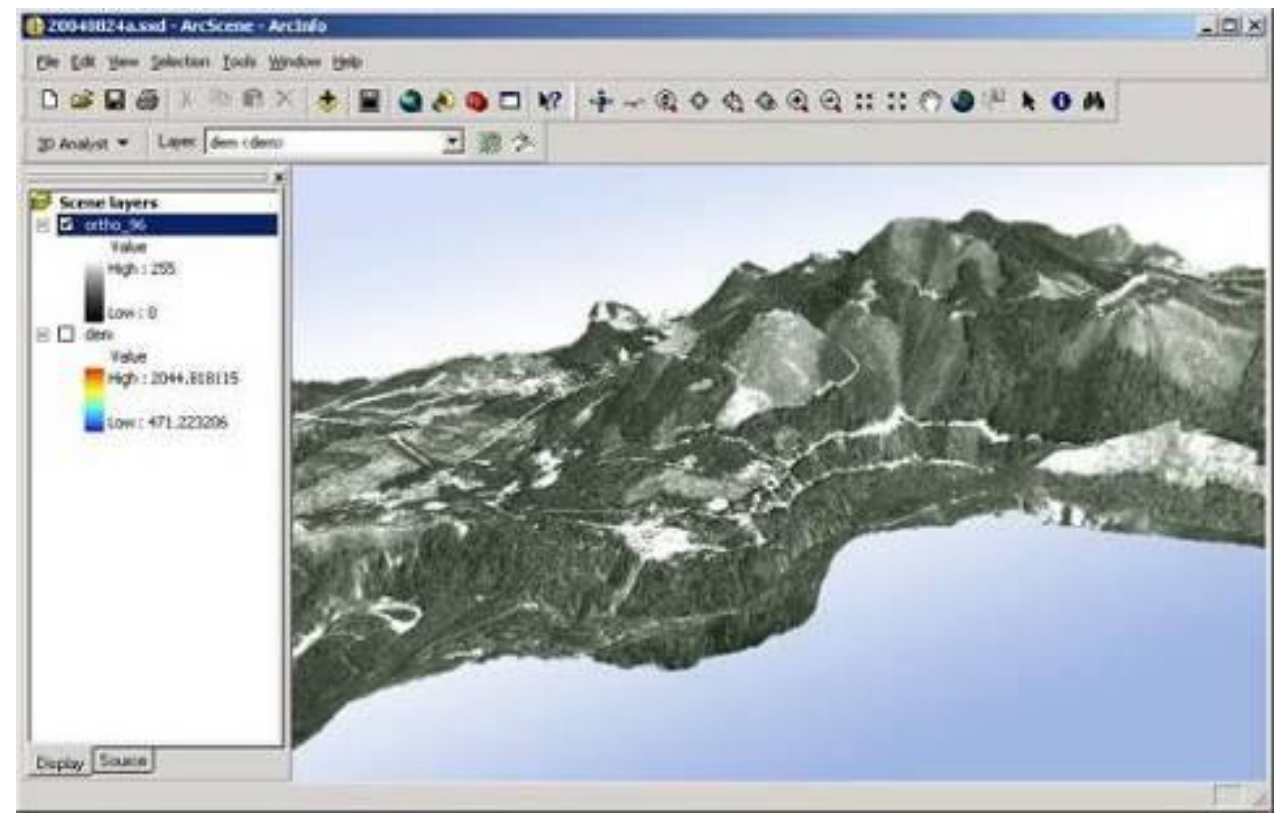

Figure 2.2: 3D visualisation in ArcScene (Land Trust GIS, 2010)

3D functionalities in GIS software broaden its capabilities to more advanced spatial analysis and authentic visualisation, which is suitable for some aspects of emergency management. Some examples were detailed in Section 2.4.

\subsubsection{D visualisation software}

There are wide ranges of non-GIS software which provide 3D visualisation of data. Visual Nature Studio $(V N S)$ is a typical example (3D Nature, LLC., 2010). Users can employ it for visualising certain types of spatial information such as vegetation classes, by importing GIS data and turn it into a photo-realistic image or video that 
people with different technical background can easily understand. VNS also provides tools to control visualisation directly from the GIS data, which simplifies and automates the process.

The Visualisation Toolkit $(V T K)$ is an open source software for 3D computer graphics, image processing, and visualisation (Kitware, Inc., 2010). It supports cross-platform development and provides a wide range of visualisation algorithms. A great many users with modelling expertise adopt this tool to generate virtual environments of different locations, and make further developments.

Vega Prime is an extendible platform for creating and deploying visual simulation results (Figure 2.3) (PRESAGIS, 2010b). With its cross-platform and scalable environment, Vega Prime is ideal for real-time 3D application development and upgrading. It enables the convenient fusion of existing code, and offers tremendous cost and time savings by enhancing the asset re-use. It also supports a high degree of customisation, and facilitates rapid prototyping and design of real-time 3D applications based on the extendable and plug-in architecture.

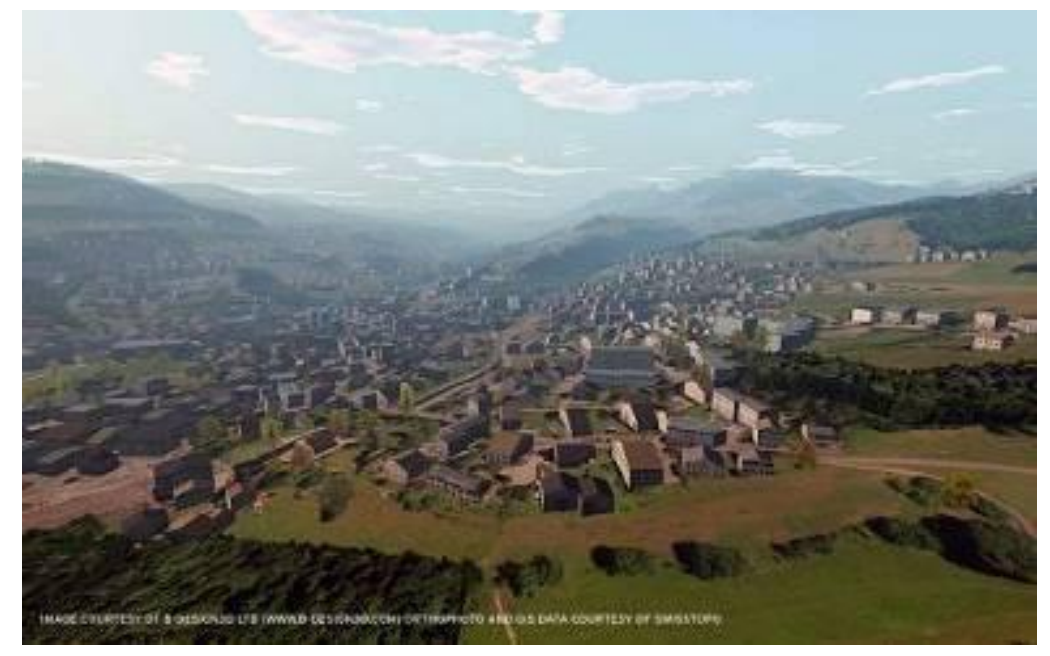

Figure 2.3: Spatial information visualisation in Vega Prime (PRESAGIS, 2010a)

It is convenient to utilise $3 \mathrm{D}$ visualisation software in emergency management. For example, Moallemi, Jafer, Ahmed, and Wainer (2011) integrated cell-Discrete Event System Specification (DES) models and robotic agents within an immersive 
environment for simulation of emergency conditions. In this system, the visualisation engine was designed using Vega Prime and OpenGL, which provided a virtual environment to verify the accuracy of the models by comparing the simulated results with the real condition.

\subsubsection{Virtual globe}

Virtual globe is a newly emerging technology that models and represents the earth in a virtual environment (Butler, 2006). The interactive capabilities enable users to explore the earth by panning, zooming and tilting. It provides not only geographical attributes like terrain elevation or satellite imagery, but also the ability to display additional information such as climate change and earthquake situation. It is growing fast in popularity as a tool to visualise and share spatial and environmental data at both local and global level.

One of the most popular virtual globes is Google Earth (Google, 2010), which shows satellite images of multi-scale resolutions of the earth's surface, and allows users to visualise entities such as buildings, looking perpendicularly down or at an oblique angle. Aerial images, road and river networks, buildings and many other layers of interest are draped over a Digital Elevation Model (DEM). Some advanced functions also include built-in flight simulator, street view, 3D bathymetry, and historical imagery. Public users can utilise Google Earth to share personal data such as photos and points of interest, while programmers can use Google Earth API for customised development and management of 3D geospatial data using Keyhole Markup Language (KML).

Another popular tool is WORLD WIND released by National Aeronautics and Space Administration (NASA). It is believed to be the first widely publicised virtual globe. It provides similar functionalities as Google Earth, such as multi-layer data and global to local area visualisation (Figure 2.4). In addition, it is enhanced by the capability of browsing maps and geospatial data via the Internet using WMS servers, supporting 
DirectX 3D polygon mesh models, as well as adding advanced visual effects such as atmospheric scattering or sun shading. More importantly, it is open source software which could be customised, modified and improved by other developers (NASA, 2010).

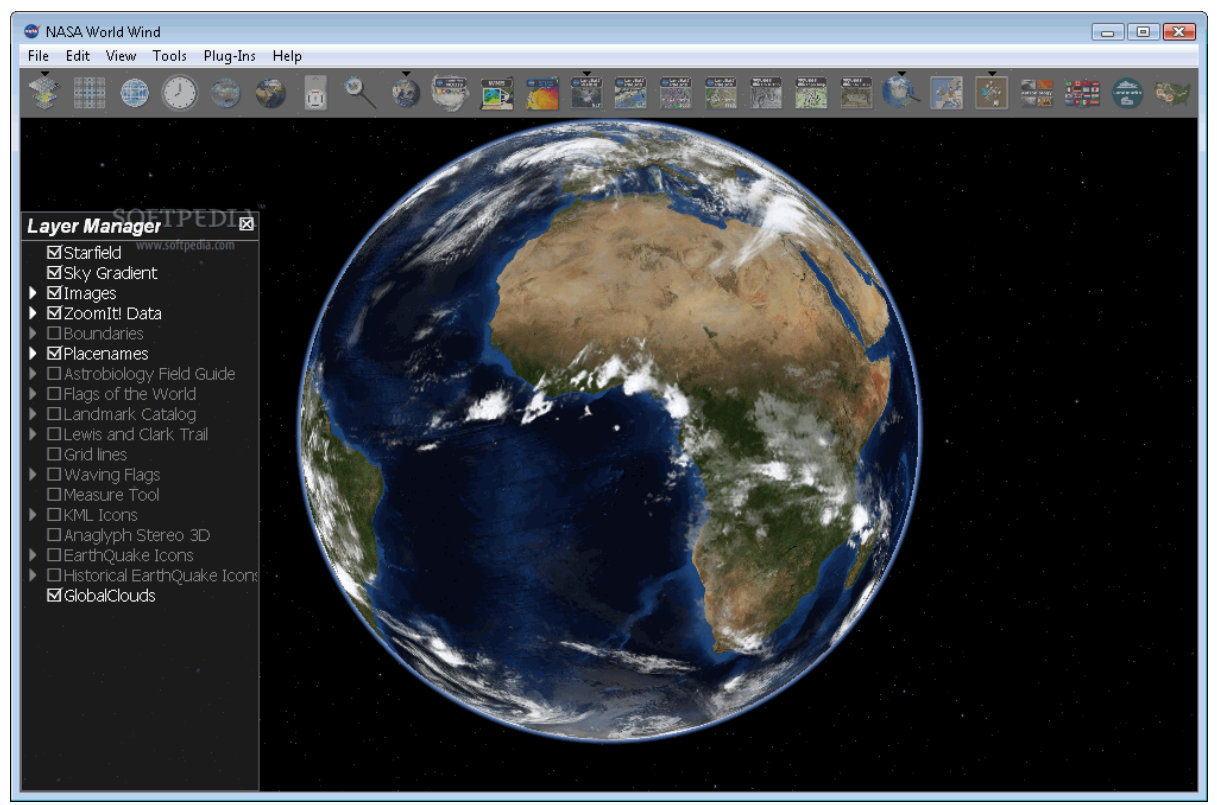

Figure 2.4: NASA's WORLD WIND (NASA, 2010)

ArcGIS Explorer is a lightweight virtual globe application. It gives users an easy way to explore, visualise, and share GIS information, and it is closely connected with other GIS software like ArcGIS Server, ArcIMS and ArcWeb service. It adds values to other GIS applications because it enables users to access ArcGIS online maps and layers, add images, videos, charts and other information, and utilise spatial analysis functions such as $3 \mathrm{D}$ analysis and spatial modelling. It further allows users to import and export various GIS file formats such as shapefile, GeoTIFF, MrSID, IMG and KML (ESRI, 2010a).

Although currently available virtual globes vary in types and functionalities, they share some common features (Butler, 2006):

- Most virtual globes are web applications that involve data manipulation via downloading and uploading. 
- Virtual globes allow users to fly through the 3D space on multiple scales ranging from global to regional, and right down to street levels.

- Various types of information with spatial reference are overlaid on satellite imagery, such as place of interest, weather information and real-time traffic.

- 3D modelling is supported including buildings, terrains and sea-floors.

- Virtual globes are mostly free to use, some are open-source or come with API-provided, which is convenient for customised development.

With the recent popularity of virtual globes, new applications are emerging in the field of emergency management. The Context Discovery Application (CDA) technology was integrated with Google Earth to support emergency situation awareness in emergency management using implicit geographic information (Tomaszewski, 2011). NASA's World Wind was utilised to develop a web application for collecting and visualising hazard data in the World Disaster Risk Evaluation and Management (DREAM) project (Dyke et al., 2011). ArcGIS Explorer was used to demonstrate population distribution in a fictitious plume of radioactive contamination as part of Global Monitoring for Stability and Security (GMOSS) in Europe (Tiede \& Lang, 2010).

Virtual globes have been widely accepted by both expert and non-expert users and the friendly user-interfaces can help to expand user participation. However, there are some unavoidable issues. One is that common virtual globe software still lacks powerful geospatial analysis functions compared with other standard GIS software. Another issue is that some information integrated into virtual globe is still 2D. For instance, weather and traffic information is represented as 2D map layers rather than real weather or traffic situations at the location. This is a disadvantage comparing with 3D visualisation in which non-expert users are able to perceive the situation, and advanced spatial analysis and realistic data presentation are well supported (Figure 
2.5).
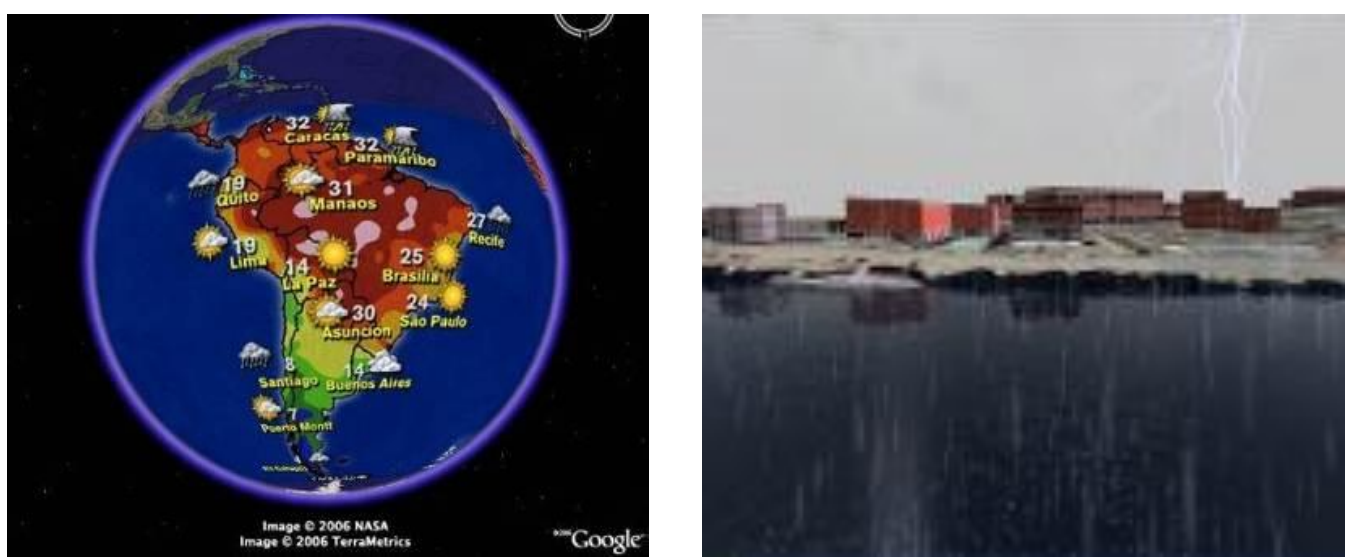

Figure 2.5: Using Google Earth and CVE to visualise weather information (GoogleEarthHacks, 2010)

\subsubsection{Current CVE development}

CVE is defined as a computer-based, distributed, virtual space which enables collaboration and interaction of many participants. People can meet and interact with other users, agents or virtual objects in a specific environment (Churchill, Snowdon, \& Munro, 2001).

Compared with virtual globes, which mainly represent global scenes using a 2D and 3D mixing method, CVE provides a more immersive environment and interactive user interface. Typical examples of CVE are distributed simulations, 3D multiplayer games, and collaborative engineering software. In fact, CVE has been widely used in diversified areas. One example is an e-tourism prototype named "itchy feet", which used a community-driven approach enabling travellers to share travelling experiences and to comment on tourism destinations in an e-tourism virtual environment (Gärtner, Seidel, Froschauer, \& Berger, 2010). VR shop is another application using CVE (De Troyer et al., 2007), in which all the commodities could be displayed as 3D format objects. Shop managers were free to add, remove and rearrange products, and customers could choose products in an interactive 3D environment.

Recently, CVE has been combined with GIS systems in the field of 
spatial-information sharing, 3D modelling, and real-time data display. A representative example is HorizoN Sentry (Branco, Escalada \& Santos, 2005), which is a real-time 3D system that performs environmental simulation with GIS data. It provided fully integrated and adaptable solutions that can be used for different purposes, ranging from urban planning to civil protection. Another example is the GRIFINOR platform (Bodum, Kjems, Kolar, Ilsøe, \& Overby, 2005). It enabled navigation in a $3 \mathrm{D}$ virtual environment with different functions for emergency management, utilised distributed computing facilities in a real-time geo-visualisation system, and combined these applications with a series of analytical tools.

\subsubsection{SIEVE overview and update}

SIEVE, or Spatial Information Exploration and Visualisation Environment, is a visualisation tool which enables users to view current spatial data and future scenarios in a 3D environment (Stock et al., 2008). It was developed by Cooperative Research Centre for Spatial Information $(C R C-S I)$, the University of Melbourne and other partners, to integrate 3D CAD models, GIS, a geo-database and a game engine. Users could select an area, such as a catchment, a group of buildings, or a town from a 2D map, and then a 3D virtual environment would be built from that area for users to view the effects of environmental processes (Figure 2.6). As SIEVE was developed locally, it is a clear candidate for use in this research and is therefore described in more detail than the preceding systems.

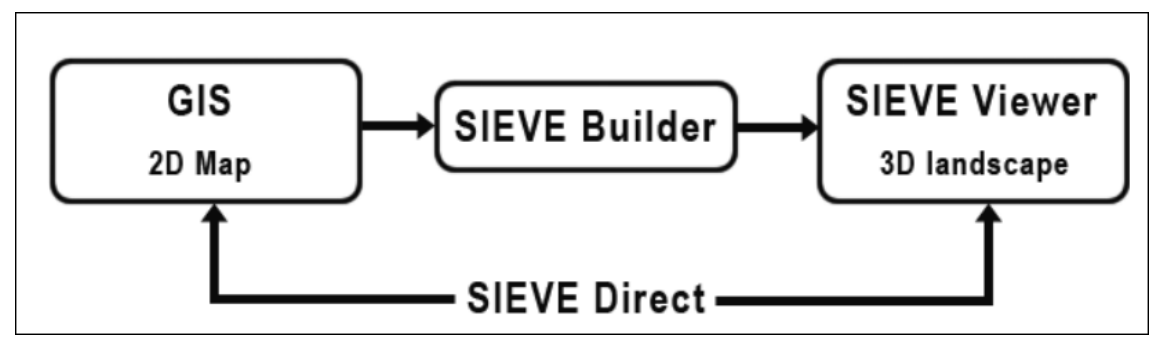

Figure 2.6: Connecting GIS and visualisation using SIEVE (Stock, Bishop, Sharma, \& Chen, 2010)

SIEVE provided network functions, therefore multiple users could explore the 
environment together. Communication options, such as a dialog box, as well as abstract and realistic visualisation options, might help people with different levels of technical competency to explore the data and make decisions in the virtual environment collaboratively. The system was mainly composed of three modules (O’Connor, Stock, \& Bishop, 2005; Stock et al., 2008):

1. SIEVE Builder: This component was developed as an ArcObjects module in VB script for ESRI ArcMap, and it was used for converting 2D GIS data (e.g. aerial imagery, DEM model, location data of building, vegetation) into a 3D data format (Figure 2.7). With this Web-based builder, users could select their areas of interest, and then a corresponding 3D model of this area would be automatically created. Once this conversion was finished, users could download the 3D model and visualise it in the SIEVE Viewer. SIEVE Builder was able to convert raster layers including DEM and satellite image into terrain models and ground texture. It was also capable of transferring vector layers including point, line, and polygons into 3D objects such as trees and buildings.

2. SIEVE Viewer: This component was developed from GarageGames' Torque Game Engine (TGE) which featured real-time rendering, virtual world editing and multi-user collaboration. SIEVE Viewer was designed as a visualisation platform displaying 3D objects which were converted from 2D data by SIEVE Builder. Additional functions were also developed to improve users' immersive experience, collaboration and navigation capability.

3. Live-link between GIS and SIEVE Viewer: This application was specially designed for transferring bidirectional messages between the GIS client and SIEVE Viewer. Users could add, delete or replace objects in a 3D virtual environment simply by manipulating data in the 2D GIS interface. Meanwhile, the GIS client could also receive messages from SIEVE Viewer and display the corresponding results in the 2D map. Additional functions sourced from this link included monitoring users' movements in the $3 \mathrm{D}$ virtual world from a $2 \mathrm{D}$ map and 
querying an object in the 3D environment.

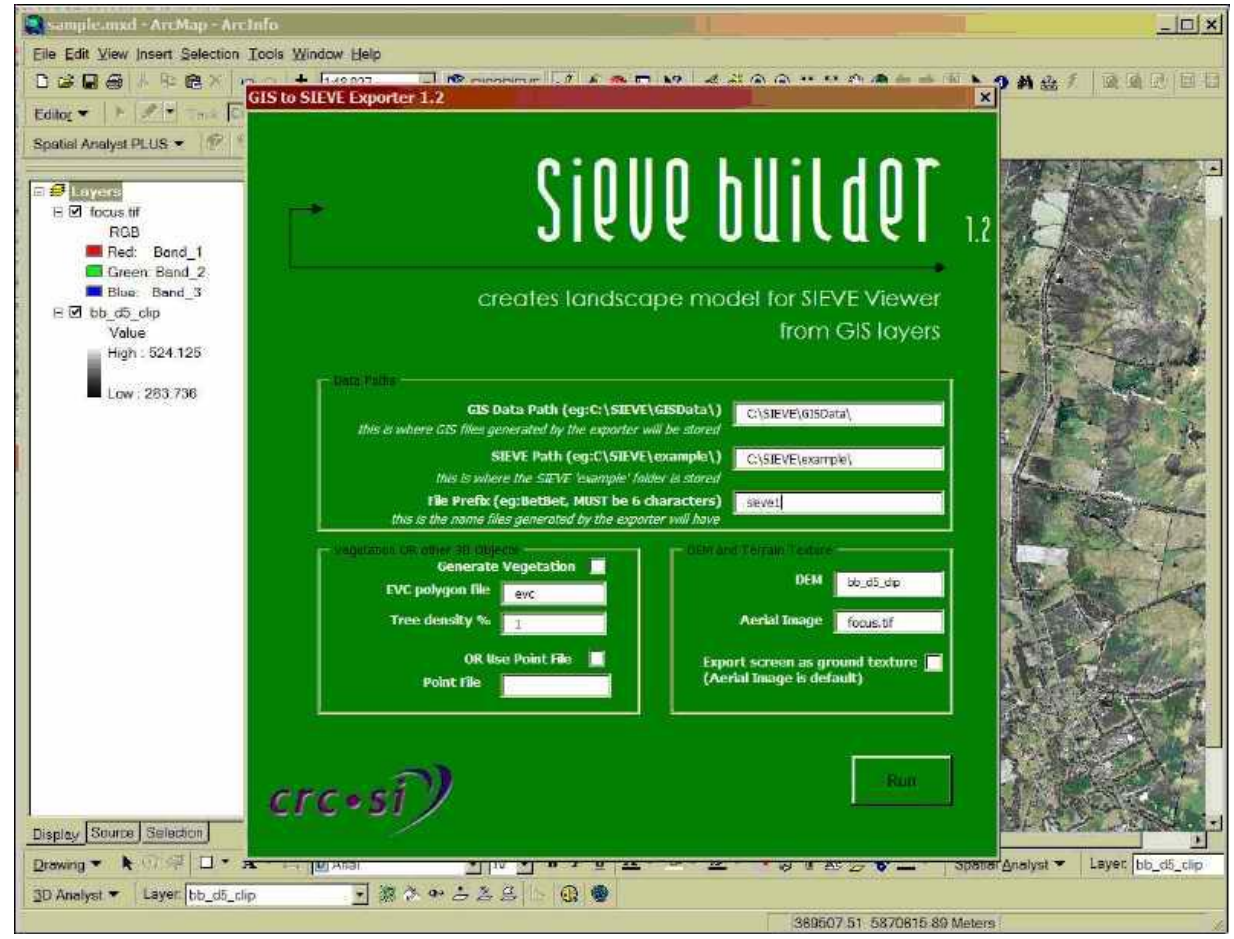

Figure 2.7: SIEVE Builder interface (O’Connor, 2007)

Recent development of SIEVE involved several applications including: Augmented Reality (AR), intelligent-farming and climate change. As an advanced extension, AR technology was adopted into SIEVE in order to provide in-field workers with visual representation of data. The AR component rendered the augmented structures from an on-line 3D object library and overlayed them onto a real world view captured by a digital camera. This would support users in land planning and management, in-field data correction and updating, as well as communication between the on-site AR and the off-site VR (Chen, Stock, Bishop, \& O’Connor, 2006).

SIEVE mainly manages static objects in the virtual environment, such as terrain, buildings and vegetation. Although SIEVE supports some dynamic simulations such as long-term changes of growing forests and short-term animations like vegetation swaying in the wind (Chen, Stock, Bishop, \& Pettit, 2008), more complicated scenes involving human activity, time-varying events and user-environment interactions are 
difficult to be integrated for higher-level simulation. For example, in virtual emergency training, real-time weather and traffic data can provide background information of the emergency situation, but it cannot be appropriately represented in SIEVE. In order to represent this data, further functionalities such as dynamic data collection, modelling and visualisation are necessary.

\subsection{Real-Time Data and Related Technologies}

\subsubsection{Real-time data}

Real-time data often refers to data delivered immediately after collection. There are four steps concerning real-time data transaction, namely: data collection, data distribution and storage, data analysis or calculation, and data visualisation. Real-time data is of significant importance for emergency management. Data of sub-optimal quality supplied in the first hour might be more useful in terms of saving lives and reducing damages, when compared with trusted, high-quality data received two days later (Zlatanova \& Nayak, 2008).

One of the most representative real-time data examples is Global Positioning System (GPS) data, or more precisely, data collected by a vehicle GPS device. The overall process of GPS transmission can be analysed based on the transactions of real-time data as described below:

- Data collection: 24 satellites are well-distributed in the space above the earth. They transmit messages containing time stamp, accurate orbital and positional information, the general system condition and orbits of other satellites. An antenna in the GPS receiver is tuned to the right frequencies and receives information from three satellites for positioning, and another one or more satellites for precision adjusting.

- Data storage: A GPS device usually contains a data caching module which records recently received data into a block for temporary storage. The cached data can 
then be utilised to calculate the direction and speed of the GPS receiver, which is helpful for vehicle navigation. Permanent data can be stored into a GPS data logger, which can be used for long trips and commercial tracking.

- Data calculation and adjustment: a GPS receiver uses the received information, records the transit time, calculates the pseudo distance to each satellite as well as the variance ratio, and demodulates the satellite orbit data. Finally, the micro-processing computer embedded in the GPS device determines the user's location information including position, direction and speed.

- Data visualisation: the location information is displayed on the screen of a GPS device. Taking "TomTom go 500" as an example, the real-time location of the user is shown in the centre of a virtual 2D map. Information such as direction, speed, time, signal intensity and other navigational data is displayed on the side of the screen. A user can follow the directions provided by the GPS device and make right decisions based on other information on the screen.

Latest weather information is another example of real-time data. Traditional methods of collecting the newest weather data involve manually copying weather information at the station to be sent back to the information processing centre. A more recent weather information collection and transition system has now been designed, using wireless data transmission via Code Division Multiple Access (CDMA) (You, Liu, \& $\mathrm{Xu}, 2010)$. The details of data collection, storage, analysis and display are described next:

- Data collection: Data collection starts at weather stations distributed in the area of interest. Weather sensors record sunlight, temperature, atmosphere pressure, wind speed and direction, humidity and precipitation, and then transfer the physical parameters into numeric data. 
- Data transition and storage: A meteorological data collector has functions including real time assembly, automatic storage and processing, instantaneous display and feedback. It can process the meteorology data from the sensors and transfer it to a central Packet Data Serving Node (PDSN) via CDMA networks using a wireless Data Terminal Unit (DTU). The PDSN can be regarded as a connection point between the DTU and the Internet. Finally the data is transferred and stored into a central server.

- Data analysis and calculation: Information obtained from the observation is integrated into a numerical model which contains some data from previous observations, to calculate future weather conditions. Using a consensus of forecast models can help to increase the forecast accuracy.

- Data display: Weather information can be displayed in many ways. Thematic mapping is one typical method of displaying weather information. Various symbols are used to represent different themes such as rainfall or temperature, while colours are used in different areas to indicate variation in quantity.

Time loss through collecting weather data from the station, updating data and calculation on the central server is unavoidable. The transition time usually lasts from seconds to minutes in real conditions. Therefore, most real-time data discussed in this section is in fact near real time because immediate real-time data is usually unachievable. Nonetheless, it is assumed that the data is real-time as long as it is still valid at the time received and hence useful for end users.

\subsubsection{Real-time data collection and distribution}

Data collection is the first step of obtaining real-time data and delivering it to end users. Varies types of sensors are utilised to capture real-time data including weather, traffic condition, and instant locations of human and objects.

Related research focused on the collection of real-time data using different tools, such 
as satellites, distributed sensors, mobile phones, GPS, video cameras, unmanned aerial vehicles (UAV), and radio frequency identification (RFID) (Yu, Wang, \& Meng, 2005; Kanjo et al., 2007; Davison, 2003; Zhou, 2009; Lin, Rowaihy, Bolbrock, Cao, \& Porta, 2008; Shao et al., 2009). Research was conducted aiming to solve the issues of data interoperability and standardisation, particularly focusing on how to organise and utilise diverse data from different sources and in different formats (Botts, Percivall, Reed, \& Davidson, 2008). Fast and automatic collection methods could help users receive real-time data with minimum delay. Data quality including accuracy, completeness, and timeliness were also enhanced. The common grounds shared by these applications are: data is directly collected by sensors, and delivered to the end users.

With the development of information technology and recent Web 2.0 applications, indirect data collection has provided another option. There are increasing numbers of content providers who publish and update their data over the Internet. In this way, real-time data can be redistributed more frequently. If users want to acquire up-to-date information, it is not necessary to collect data from scratch. Instead, data can be collected from other sources in near real time.

Web application hybrid or mashup, refers to a web application that integrates data from different sources into one single tool. As a newly emerging technology, it plays a very important role in Web 2.0, emphasising a more interactive, participatory, collaborative process for users to explore the Internet. The advantage is that users who wish to collect data do not have to do it from the beginning, for there are mass data emerging on-line with thousands of vendors publishing data to the public and private sectors. Users who have basic programming experience are able to develop their own web applications by implementing APIs such as Google Maps or Amazon eCommerce, and by appending existing on-line data like real-time weather or traffic information from heterogeneous sources. 
An important tool for mashup is the on-line map, especially Google Maps, which provides web developers with convenient functions to add location-annotated information onto maps. Once a data set has been labelled with location-related information such as latitude and longitude or address, developers can easily assemble such data into Google Maps to form new applications. There have also been other genres emerging in recent years, such as photo, video, search and shopping mashup.

A mashup application (Figure 2.8) is usually composed of three parts: data/content providers, the mashup site, and the user-interface (Merrill, 2006):

- Data/content providers: Data providers publish data that can be mashed up by the developers. They open their content to users via web-protocols such as SOAP and REST. For example, in the mashup application "Weatherbonk" (The Weather Channel, LLC., 2010b), the providers are The Weather Channel (The Weather Channel, LLC., 2010a), Citizen Weather Observer Program (Chadwick, 2010), and the National Weather Service (NOAA, 2010a). The weather information is pre-processed and published via its own Web service.

- The mashup site: This is where the mashup is hosted. Mashup can be realised similar to other web applications using server-side dynamic content generation technologies such as Java servlets, CGI, PHP or ASP.

- User-interface of mashup: This is where the result is displayed and user interaction occurs. Technologies such as HTML/XHTML, CSS, Ajax are applied here. 


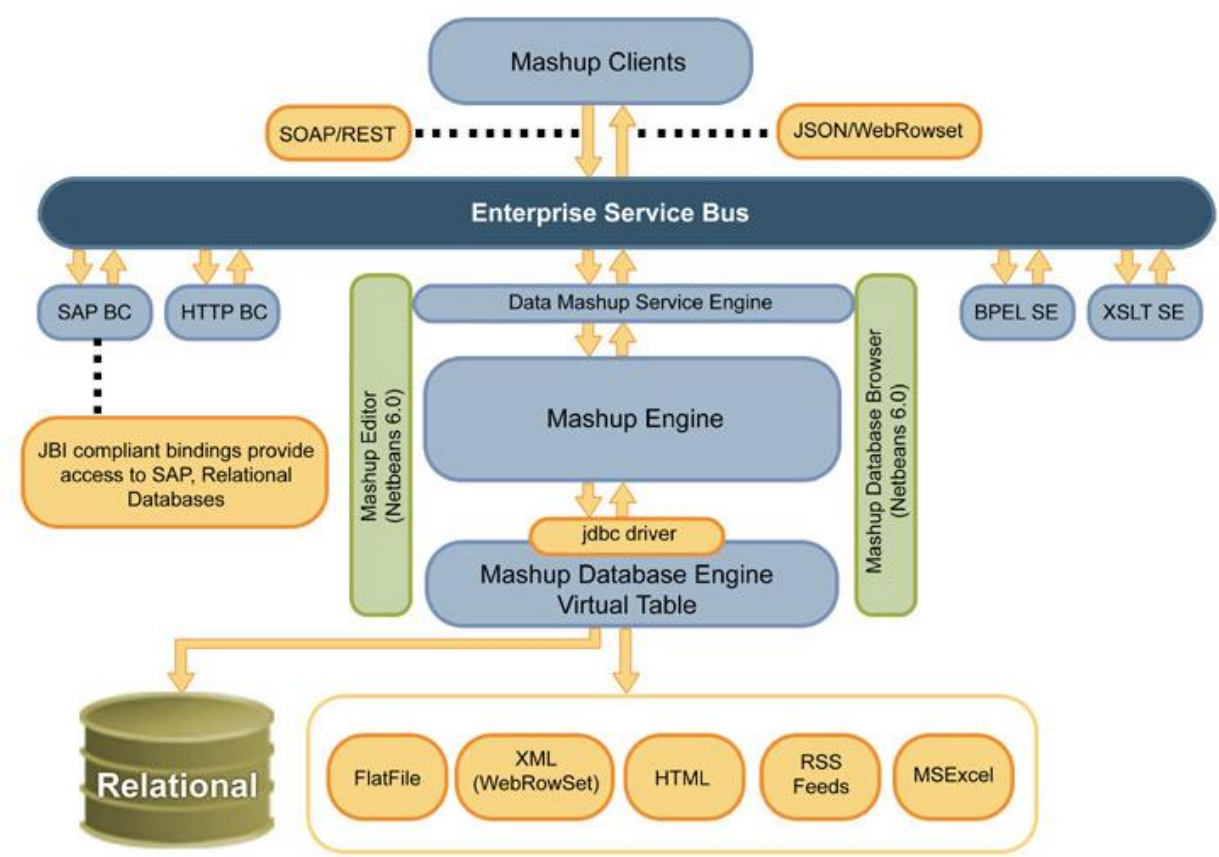

Figure 2.8: Architecture of mashup technology (Oracle, 2010a)

One example of mashup is "alkems local" (Alkems, 2010). Users can discover useful datasets including weather, real-time traffic, and subway live traffic cams information which can benefit their trip planning. In addition, up-to-date news from "Yahoo!" and $C N N$, as well as trading information from eBay and Amazon are available to users. With Ajax and JSON related technology, this application provides users with comprehensive information from different sources in a common user interface.

"WikiCrimes" (Wikicrimes, 2010) is another well-known mashup application, which is a wiki-style website. Users can report crime incidents by adding labels on Google Maps. Some users can report true experiences of crimes while others can access the crime location and types so as to avoid such dangers. Social scientists can also refer to the data for statistical purposes. It not only involves users' browsing but also engages their participation and interaction.

\subsubsection{Real-time data modelling and visualisation}

Real-time data is a special type of time-varying data, the content of which changes 
with the time series. When it is linked with a spatial attribute, real-time data also can be considered as spatial-temporal data, with the temporal attribute as current time. Spatial-temporal data has three components: spatial attribute, temporal attribute, and descriptive attribute. Take a fire incident happening in Sydney Harbour as an example: Sydney Harbour, or the specific location where the incident happens, is the spatial attribute, the time during which the incident occurs is the temporal attribute, and incident type and status are the descriptive attributes.

Conventional spatial information is represented in static maps. This method faces difficulties when displaying dynamic process such as urban area development or real-time traffic. Appropriate models should be selected for simulating the change of spatial information with time. Theoretical models have been proposed and meliorated, resulting in several research topics. Some models represent spatial-temporal information using time-stamping spatial objects, such as: the snapshot model (Armstrong, 1988), temporal map sets (TMS) (Beller et al., 1991), the Space-Time Composites Model (STC) (Langran \& Chrisman, 1988), and the spatiotemporal object model (ST-Object model) (Worboys, 1992). Some represent the information by events and process, such as the event-based spatial temporal model (ESTDM) (Peuquet \& Duan, 1995), and the geomorphologic spatial model (OOgeomorph) (Raper \& Livingstone, 1995).

In addition to these theoretical models, some practical applications in modelling and visualising spatial-temporal data have been developed. Goldsberry (2008) introduced new methods of visualising real-time traffic information using established cartographic principles such as classification schemes, symbolisation schemes, and representational forms. These methods exposed the issues currently existing in online traffic maps, and identified the most significant design variables which influenced user perception. Tominski, Schulze-Wollgast, and Schumann (2005) described a method of visualising spatial-temporal data using maps, where discursive 3D pencil and helix icons capable of visualising various time-dependent data were designed and 
embedded into a map display. It ameliorated an event-based approach, and was able to visually guide users' attention to focus on events of interest while hiding irrelevant information. Yu, Lu, Ribarsky, and Chen (2010) presented another approach that generated automatic animations for time-varying data visualisation. It simulated the composition and transition of story-telling techniques and synthesised animations to describe various event features. It was achieved using the concept of event graph, which abstracted event features from different aspects and scales, as well as an automatic animation process with a series of event detection, time segmentation, graph searching and rendering composition methods. Aigner, Miksch, Müller, Schumann, and Tominski (2007) summarised the methods of visualising time-oriented information, and developed an open framework for representing time-oriented data. Important features of visualising time-oriented data were emphasised and included: multiple views for different data aspects, sophisticated and adaptable interactions, and tighter integration of visual and analytical tools. ESRI (2010c) produced ArcGIS Tracking Analyst as one of its desktop extensions. It enables users to visualise and analyse spatial-temporal data. The main features include real-time data visualisation, time-based symbology, and playback. However, the lack of immersive 3D visualisation support prevented its application in this research.

There are also some studies underway attempting to model and visualise spatial-temporal data in 3D environments such as virtual globes or CVE. Bista and Pack (2008) developed a method to dynamically demonstrate and interact with the real-time simulation result based on a random microcosmic traffic simulation model in $4 \mathrm{D}$. It could retrieve data from real time sensors, display the current traffic flow and predict the immediate future traffic conditions over an area. Sharkawi, Ujang, and Abdul-Rahman (2007) described the development of a 3D navigation system by utilising a freely available 3D game engine coupled with GIS elements. It aimed to extend the functionalities of game engine and incorporate it with mobile devices for real-time positioning. Castrillón et al. (2011) further described a wildfire forecasting software using a 3D virtual environment combined with a fire simulation model and 
GIS. It integrated spatial information on topography and up-to-date meteorological information such as weather and wind data. The system could achieve a realistic composition of dynamic 3D objects, as well as the location of human and material resources in real time. It described what actually happened in the wildfire area and provided a novel perspective of analysing wildfire information. Shiode and Yin (2008) suggested a spatial-temporal 3D-GIS city model, which created a hybrid 3D city model from CAD and GIS. The model was realised in ArcScene, with a slide bar controlling the time lapse, and each 3D building assigned temporal attributes. Users could therefore follow the spatial temporal dynamics of an urban environment development. The concept of temporal links was raised to build a flexible mechanism for recording past or recent events in a CVE (Greenhalgh et al., 2000). It combined temporal, spatial and presentational properties. Temporal properties controlled the relationship between time in a virtual environment and in reality. Spatial properties defined the spatial relationship between the CVE and the recording. Presentational properties determined the user-interface of the virtual environment.

In the context of emergency management, visualising and delivering spatial-temporal data needs to be completed in a timely manner. A wide range of emergency contexts have been considered as appropriate for development of real time visualisation. A cartographic web application was designed based on a viable data model, and allowed users to view the past and current hydrological situations in automatically processed real-time maps with different levels of detail. This application generated great benefits for flood monitoring and warning (Lienert, Kunz, Weingartner, \& Hurni, 2010). The Transportation Incident Management Explorer (TIME) was implemented to visualise both historic and real-time urban traffic incident data with spatial and temporal attributes. TIME reduced the possibility of missing important information and facilitated quick and accurate decision making during the incident management process (VanDaniker, 2009). Severe weather condition data with a high temporal and spatial resolution was generated and integrated into a virtual globe display, therefore the warning data could be efficiently delivered to the end users, including emergency 
managers, response teams and the public (Smith \& Lakshmanan, 2011).

\subsection{Summary}

This chapter reviewed emergency management and related technology. The two important activities in emergency management were focused: emergency monitoring and emergency training. Emergency monitoring plays an important role in emergency response, and involves technologies such as remote sensing, mobile devices and sensors. Traditional training methods and virtual training using CVE have been discussed and compared with the conclusion that virtual training has many advantages such as high-fidelity simulation, lower risk and greater flexibility and convenience.

The integration of a 3D virtual environment has greatly benefited emergency management. Recent CVE developments, especially video game technology, have shown strengths in visualising and simulating data, as well as tactics proposing. Moreover, spatial information plays a critical role in emergency management. It not only plays an important role in the fields of urban planning, logistics, population and demographic studies, but also provides techniques facilitating the application of novel models in emergency management. Importantly, there is great potential for real-time spatial information to be collected, distributed, analysed, modelled and visualised in emergency management, to enhance users' understanding and communication.

In the real situation of emergency management, the ongoing development of real-time data integration technology raises new possibilities. Mashup, as a representative example, has been demonstrated effective in various use cases. These applications are primarily $2 \mathrm{D}$ and map-based, while this research migrates this technology from $2 \mathrm{D}$ maps to $3 \mathrm{D}$ virtual environments. The time attributes of emergency management are also significant as the spatial and temporal representation of incident data is a key task for this work. The remainder of this thesis will describe how emergency management can be improved by well-organised integration of multiple technologies. 



\section{Chapter 3 System Design and Methodology}

\subsection{Introduction}

Chapter 2 outlined the trends in geo-spatial information, virtual reality, real-time data collection and visualisation technology. Geo-spatial information technology can effectively manage location-based data in terms of collection, storage, analysis and visualisation, thus providing significant assistance to decision makers when planning and executing emergency management strategies. Virtual reality techniques can greatly reduce the costs involved in field exercises and can significantly improve the performance compared with other desktop-based exercises. Real-time data can be collected and visualised in simulation systems, providing users with useful insights into the on-site situation. The literature review and relevant findings from recent research were used to formulate the foundation of this research.

This chapter describes the design and development of a prototype system, SIEVE-VTS, or the Spatial Information Exploration and Visualisation Environment - Virtual Training System. It aims to effectively deliver and visualise marine-related real-time information in CVE, thereby enhancing the authenticity of the scenario, improving the usability for emergency management, and strengthening collaborations among users. SIEVE-VTS comprises a combination of proprietary software and custom modules, including a real-time data collector, a data translator, a data simulator, as well as an event-based database. Section 3.2 introduces the performance and functional requirements of the system based on related research and the potential needs of users. Section 3.3 introduces the real-time data types utilised for this research. Section 3.4 outlines the technical foundation, the overall architecture, and components of the SIEVE-VTS prototype system. Section 3.5 describes the functions of the real-time data collector, or how real-time data is streamed from information providers to the event-based database. Section 3.6 introduces the functions of the real-time data translator, which converts real-time information from its original data formats to new 
data formats. Section 3.7 illustrates the real-time data simulator based on TGEA and the user-interface facilitating emergency scenario simulation. Section 3.8 addresses the problem of displaying information in areas where no real-time data is available. The last section summarises this chapter and discusses the virtues and limitations of the system.

\subsection{System Requirement}

System requirements involve determining the needs or conditions for a new or altered product, taking into account the users' requirements. In this case, these requirements include those derived from related research and those specified by the clients of this project. Specific system requirements have been developed (Table 3.1).

- Both static and dynamic scenarios can be built and simulated in affordable time.

- Real world spatial information should be accurately integrated into this system, therefore users can efficiently locate themselves and virtual objects, such as vessels and vehicles.

- The user experience in the virtual environment needs to be highly authentic.

- The system can be utilised for monitoring onsite emergency situations using real-time data.

- The system can help emergency training using historical data to simulate past incident scenarios.

- The system should support multiple user collaboration through a network.

- Rendering time for the scenario in virtual environments is acceptable.

- Total costs of system design and usage should be attractive when compared with other software addressing similar issues.

Table 3.1: System requirement

The developed SIEVE-VTS can be utilised by diverse user groups. The primary users are project collaborators from Australian defence organisations including Defence Science and Technology Organisation (DSTO) and Defence Imagery and Geospatial Organisation (DIGO). SIEVE-VTS has been developed as a cost-efficient, easy to 
update simulation platform, focusing on emergency and defence simulation in outdoor and indoor environment.

\subsection{Data Selection}

The system requirements place significant emphasis on real-time data because of the anticipated benefits to emergency management. Real-time data can enhance the authenticity of emergency training and support decision makers in monitoring the area of interest. Furthermore, large amounts of real-time data are available and accessible either from online Web services or directly from data providers, which makes it possible to integrate real-time data into SIEVE-VTS.

In this research, four representative types of real-time data were selected: weather condition, oceanographic data, Automatic Identification System (AIS) data, and incident location and status.

- Weather condition: Key parameters of real-time weather include temperature, atmospheric pressure, wind status, visibility and sunshine. Understanding weather condition provides basic environmental information about an area, while different weather conditions may have impacts on preparedness and response during emergency management. For example, discovery and control of an emergency incident is more difficult during harsh weather with low visibility than in fine weather with clear conditions. Data can be obtained from many public websites, such as Bureau of Meteorology, Australia (BOM, 2010b) and Yahoo! Weather (Yahoo! Inc., 2010).

- Oceanographic data: Various types of oceanographic data are available, such as ocean currents, salinity, wave, tide, and surface temperature. In this research, wave and tides were selected as the representative oceanographic data. Such data can provide marine environmental and ecosystem information in harbour and offshore, which is the focus area of this research. Data is distributed by BOM or NOAA (NOAA, 
2010b) and other agencies.

- AIS: AIS is an automatic tracking system for ships. It records ship information including Maritime Mobile Service Identity (MMSI), position, speed, and course. Using AIS data to simulate shipping enables users to implement plans and strategies during emergency training as well as harbour security monitoring. Data is available from on-line content providers and DSTO.

- Incident location and status: Up to date information of incident locations and status, such as fire or explosions are collected. Details include: location, time, incident type, scale, and status. The frequently updated information provides users with real-time states of incidents, assisting users to quickly respond to the incident. Data can be collected by safety guards, police or other observers who experience the incident.

Data mentioned above does not include the entire real-time data sets essential for emergency management. With the availability of other kinds of real-time data increasing and new technologies being promoted, SIEVE-VTS could be populated with more types of data, such as real-time traffic conditions in urban areas and live webcams for instantly monitoring situations.

\subsection{Overall Design of SIEVE-VTS}

The overall framework is based on the system requirements. The system is comprised of 3 layers: data layer, manipulation layer, and visual simulation layer. Each layer contains modules which independently provide services through standard communication protocols. In this manner, the system makes possible both sharing of spatial information and the execution of collaborative work (Figure 3.1). 


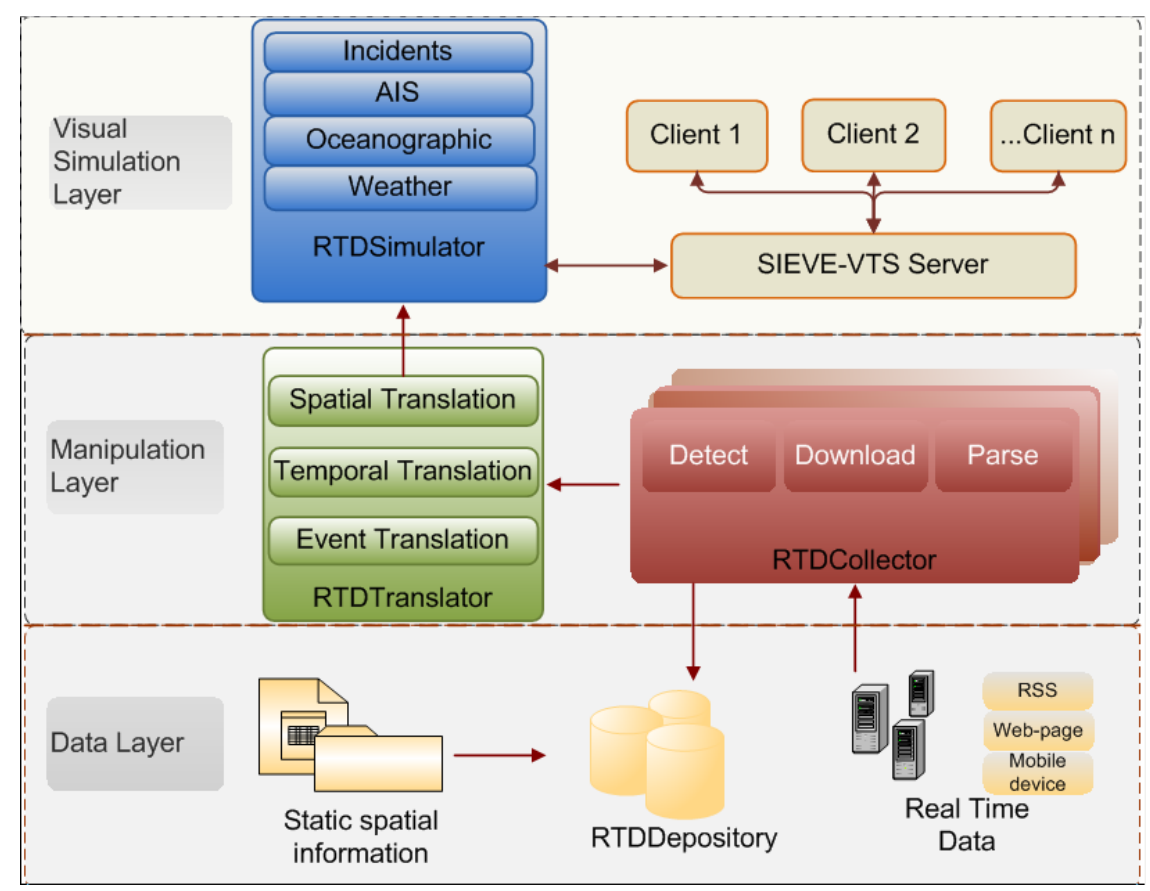

Figure 3.1: Architecture diagrams of SIEVE-VTS

- Data layer:

The data layer is composed by several types of datasets, including both static and dynamic spatial information. Static spatial data is created in advance, including terrain, buildings and vegetation. Data is stored in mission files and data files appropriate to the CVE. Dynamic spatial information includes environmental data with spatial and temporal attributes, such as local weather, oceanographic data, incident location and status. The original data is provided by on-line content providers, either from public or private sectors. An event database named Real-time Data Depository (RTDDepository) is used to efficiently store and organise real-time data.

- Manipulation layer:

The manipulation layer is mainly formed by two modules. A Real-time Data Collector (RTDCollector) automatically monitors the state of on-line real-time data, captures it when there is new data available, and parses the information into datasets which can be conveniently stored into the RTDDepository. A Real-time Data Translator 
(RTDTranslator), as the middleware, translates original real-time data into a format which is recognisable and can be easily visualised by the Real-time Data Simulator (RTDSimulator).

- Visual simulation layer:

The visual simulation layer is composed of the RTDSimulator which instantaneously receives data from the manipulation layer, and visualises it in a Collaborative Virtual Environment underpinned by a game engine. RTDSimulator can demonstrate static scenarios using pre-defined data created by other assistant tools, and simulate dynamic scenarios using real-time data collected from on-line content providers. The module is also implemented as a client-server architecture which facilitates users' collaboration in the virtual environment.

Based on the prototype designed above, SIEVE-VTS is implemented by adapting existing software using an object-oriented programming method. The selected software utilises Torque Game Engine Advanced (TGEA) (GarageGames, 2010b) as the main simulation platform and MySQL (Oracle, 2010c) with its spatial extensions as the central database.

TGEA was selected to be the main simulation platform because of its certain advantages over other existing virtual simulation software (Table 3.2). Its built-in atlas terrain system with terrain paging management can create smooth and seamless terrains of arbitrary size; its embedded physics engine supports basic physics, rigid body, and collision detection; its water engine can effectuate shoreline textures and dynamic waves; its lighting and shadowing system improves the light and shadowing effects dramatically. These functionalities make it an ideal tool for real-world-like scenario simulation including both natural and anthropogenic phenomenon. TGEA uses client-server architecture, which provides CVE enabling users to explore the same environment from different physical locations using Internet or LAN. TGEA also provides its license at a relatively low price, which reduces the system cost 
dramatically compared with other emergency management tools. The Graphic User Interface (GUI) creation toolset, in-game editors, scripting language and full access $\mathrm{C}++$ source code will allow the engine to be easily customised and developed.

\begin{tabular}{|l|l|l|l|l|l|}
\hline \multicolumn{1}{|c|}{ Platform } & $\begin{array}{c}\text { Initial } \\
\text { Release }\end{array}$ & $\begin{array}{c}\text { Physics } \\
\text { Engine } \\
\text { Supported }\end{array}$ & $\begin{array}{c}\text { Development } \\
\text { Feasibility }\end{array}$ & CVE-Based & $\begin{array}{c}\text { Virtual } \\
\text { City }\end{array}$ \\
\hline Google Earth & 2005 & Weak & High & No & Yes \\
\hline Second Life & 2003 & Medium & Medium & Yes & Yes \\
\hline VBS 2 & 2007 & Strong & Low & Yes & Yes \\
\hline TGEA & 2007 & Strong & High & Yes & Yes \\
\hline
\end{tabular}

Table 3.2: Comparison of TGEA and other software

$M y S Q L$ is one of the most widely-used database systems employed by web developers, and its fast performance and cross platform capabilities make it ideal for small scale applications. In addition, the spatial extensions of $M y S Q L$ can generate, store, and analyse spatial features more efficiently. As a freely available and open source software, $M y S Q L$ also reduces the cost and is easily integrated into TGEA.

\subsection{Real-Time Data Integration}

RTDCollector is the core module for collecting, parsing and storing real-time data. It is written in $\mathrm{C}++$ and Torque Script, and encapsulated as an independent component in SIEVE-VTS. The work flow is shown as follows (Figure 3.2): 


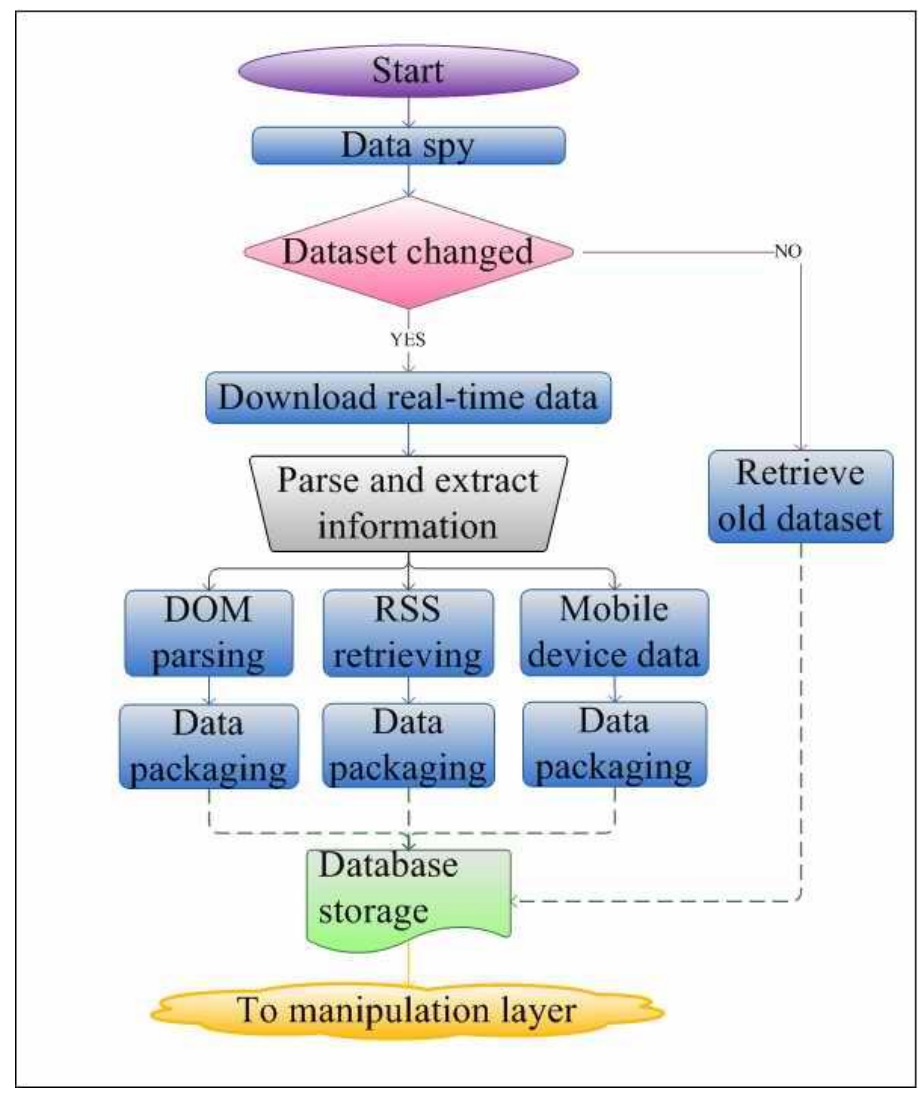

Figure 3.2: Flow-chart of RTDCollector working procedures

1. RTDCollector starts requesting a certain type of data which is customised by users through a GUI (Figure 3.3). The subsequent transaction is based on the data type defined in this step.

2. It monitors the states of a certain dataset, which is called data spy. Two different methods, active spy and passive spy are applied on different data types.

3. When it detects that data has been updated, an automatic downloading method will be triggered. If the dataset has not been updated, it will use the last historical data stored in $M y S Q L$.

4. It parses the data using different methods based on data formats, and extracts useful information from it.

5. Finally the parsed real-time data is collected and stored into $M y S Q L$ database. 


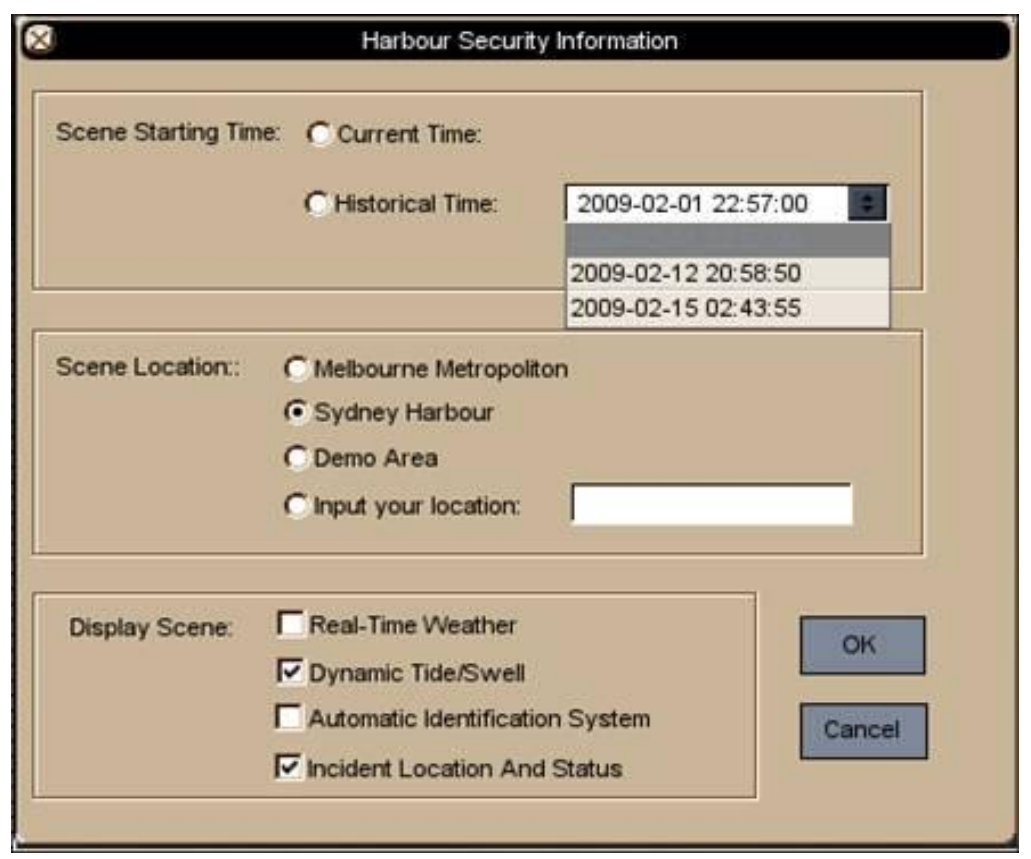

Figure 3.3: RTDCollector Graphic User Interface (GUI)

Methods of collecting real-time data can be divided into 3 categories according to the different formats of data:

a. Web scraping: Some useful real-time information is not provided in standard formats, but is simply embedded in a static webpage. Therefore it is necessary to design a data structure that fits the datasets in the webpage, and retrieve useful data from it. Individually designed procedures are required in each case as described in Section 3.5.1. In this research, weather and oceanographic data were collected using this method.

b. Mashup technology: Some data is provided in well-organised formats and is usually transferred in XML. Web services and APIs are also provided by some content providers, making it convenient for users to access the data and make further developments. The main technology involved is data mashup (introduced in Section 2.6.2 and described in more detail in Section 3.5.2). In this case, it is used for collecting AIS and auxiliary weather information.

c. On-site collection using mobile device: Sometimes there is no ready-to-use data 
provided online by public or private content providers. Therefore, it is necessary to collect onsite information using mobile devices. Currently, Personal Digital Assistant (PDA) and smart phones with built-in GPS, camera and communication modules are ideal for collecting such onsite real-time data. In this research, incident location and status were collected by an iPhone application.

\subsubsection{Real-time data integration using Web scraping}

Web scraping is a technique for extracting data from websites. It translates the unstructured web content, usually in HTML format, into structured data that can be stored and analysed in a central database. It simulates human browsing of the website by implementing low-level Hypertext Transfer Protocol (HTTP). Examples of Web scraping include online price comparison, weather information visualisation and website change detection (Jonas, 2004).

In this research, the first data type accessed using Web scraping is the weather condition. Hundreds of organisations and institutions provide local or global weather information to public or specific users. In this research, several main weather information Web services were investigated, including BOM, Yahoo! Weather, and Weatherzone (The Weather Company, 2010). The primary weather condition data is collected from $B O M$, which is the authority agency providing weather services in Australia. Many products, in a variety of formats, are available, such as climate data, weather forecasts, warnings, and ocean conditions. In this research, current weather data is the only information taken into account. In order to illustrate the process, the weather information at Sydney Harbour station is taken as an example (Figure 3.4). 


\begin{tabular}{|c|c|c|c|c|c|c|c|c|c|c|c|c|c|c|c|c|c|c|}
\hline \multicolumn{19}{|c|}{ SYDNEY AREA } \\
\hline & \multirow{2}{*}{$\begin{array}{l}\text { Date/Time } \\
\text { EST }\end{array}$} & \multirow{2}{*}{$\begin{array}{l}\text { Temp } \\
{ }^{\circ} \mathrm{C}\end{array}$} & \multirow{2}{*}{$\begin{array}{c}\text { App } \\
\text { Temp } \\
{ }^{\circ} \mathrm{C}\end{array}$} & \multirow{2}{*}{$\begin{array}{l}\text { Dew } \\
\text { Point } \\
{ }^{\circ} \mathrm{C}\end{array}$} & \multirow{2}{*}{$\begin{array}{c}\text { Rel } \\
\text { Hum } \\
\%\end{array}$} & \multirow{2}{*}{\begin{tabular}{|c} 
Delta \\
$\mathrm{T}$ \\
${ }^{\circ} \mathrm{C}$
\end{tabular}} & \multicolumn{5}{|c|}{ Wind } & \multirow{2}{*}{$\begin{array}{l}\text { Press } \\
\mathrm{hPa}\end{array}$} & \multirow{2}{*}{$\begin{array}{l}\text { Rain } \\
\text { since } \\
9 \mathrm{am} \\
\mathrm{mm}\end{array}$} & \multirow{2}{*}{$\begin{array}{l}\text { Low } \\
\text { Temp } \\
{ }^{\circ} \mathrm{C} \\
\text { time }\end{array}$} & \multirow{2}{*}{$\begin{array}{l}\text { High } \\
\text { Temp } \\
{ }^{\circ} \mathrm{C} \\
\text { time }\end{array}$} & \multicolumn{3}{|c|}{ Highest Wind Gust } \\
\hline & & & & & & & Dir & \begin{tabular}{|c|} 
Spd \\
$\mathbf{k m h}$
\end{tabular} & $\begin{array}{l}\text { Gust } \\
\text { km/h }\end{array}$ & $\begin{array}{l}\text { Spd } \\
\text { kts }\end{array}$ & $\begin{array}{l}\text { Gust } \\
\text { kts }\end{array}$ & & & & & Dir & $\begin{array}{c}\mathbf{k m h} \mathbf{h} \\
\text { time }\end{array}$ & $\begin{array}{l}\text { kts } \\
\text { time }\end{array}$ \\
\hline $\begin{array}{l}\text { Sydney - } \\
\text { Observatory Hill }\end{array}$ & $17 / 01: 25 \mathrm{pm}$ & 17.9 & - & 2.1 & 34 & 7.2 & - & - & - & - & - & 1004.7 & 0.0 & $\begin{array}{l}8.2 \\
06: 32 \mathrm{am}\end{array}$ & $\begin{array}{l}18.0 \\
12: 47 \mathrm{pm}\end{array}$ & - & - & - \\
\hline Sydney Airnort & $17 / 01: 24 \mathrm{pm}$ & 17.8 & 9.9 & 2.5 & 36 & 7.0 & W & 33 & 39 & 18 & 21 & 1005.0 & 0.2 & $\begin{array}{l}8.3 \\
03: 56 \mathrm{am}\end{array}$ & $\begin{array}{l}19.1 \\
01: 09 \mathrm{pm}\end{array}$ & W & $\begin{array}{l}52 \\
01: 21 \mathrm{pm}\end{array}$ & $\begin{array}{l}28 \\
\mathrm{~m} 01: 21 \mathrm{pm}\end{array}$ \\
\hline Sydney Harbour & $17 / 01: 24 \mathrm{pm}$ & 17.5 & - & - & - & - & W & 50 & 56 & 27 & 30 & - & - & $\begin{array}{l}10.4 \\
04: 01 \mathrm{am}\end{array}$ & $\begin{array}{l}17.7 \\
01: 08 \mathrm{pm}\end{array}$ & $w$ & $\begin{array}{l}65 \\
01: 17 \mathrm{pm}\end{array}$ & $\begin{array}{l}35 \\
\mathrm{~m} 01: 17 \mathrm{pm}\end{array}$ \\
\hline $\begin{array}{l}\text { Sydney Olympic } \\
\text { Park }\end{array}$ & $17 / 01: 24 \mathrm{pm}$ & 17.9 & - & 2.2 & 35 & 7.1 & - & - & - & - & - & - & 0.0 & $\begin{array}{l}7.4 \\
05: 38 \mathrm{am}\end{array}$ & $\begin{array}{l}18.9 \\
12: 43 \mathrm{pm}\end{array}$ & - & - & - \\
\hline Eadgervs Creek & $17 / 01: 25 \mathrm{pm}$ & 16.4 & 10.8 & 5.3 & 47 & 5.3 & W & 24 & 30 & 13 & 16 & - & 0.0 & $\begin{array}{l}3.1 \\
06: 37 \mathrm{am}\end{array}$ & $\begin{array}{l}17.6 \\
12: 18 \mathrm{pm}\end{array}$ & WSW & $\begin{array}{l}59 \\
11: 11 \mathrm{am}\end{array}$ & $\begin{array}{l}32 \\
\mathrm{~m} 11: 11 \mathrm{am}\end{array}$ \\
\hline Bankstown & $17 / 01: 25 \mathrm{pm}$ & 17.0 & 9.8 & 5.0 & 45 & 5.7 & W & 32 & 41 & 17 & 22 & 1005.5 & 0.0 & $\begin{array}{l}3.9 \\
07: 01 \mathrm{am}\end{array}$ & $\begin{array}{l}18.1 \\
\text { n } 01: 06 \mathrm{pm}\end{array}$ & $w$ & $\begin{array}{l}57 \\
01: 23 \mathrm{pm}\end{array}$ & $\begin{array}{l}31 \\
\mathrm{~m} 01: 23 \mathrm{pm}\end{array}$ \\
\hline
\end{tabular}

Figure 3.4: Snapshot of weather information from $B O M$

The webpage is refreshed automatically every 5 minutes. When a URL is requested for the first time, the content of the webpage is retrieved with a "Last-Modified" attribute label in the HTTP header. If the same URL is requested a second time, the HTTP header will be returned containing the attribute "if-modified-since". This difference can be used to determine if a webpage has been updated (Figure 3.5).

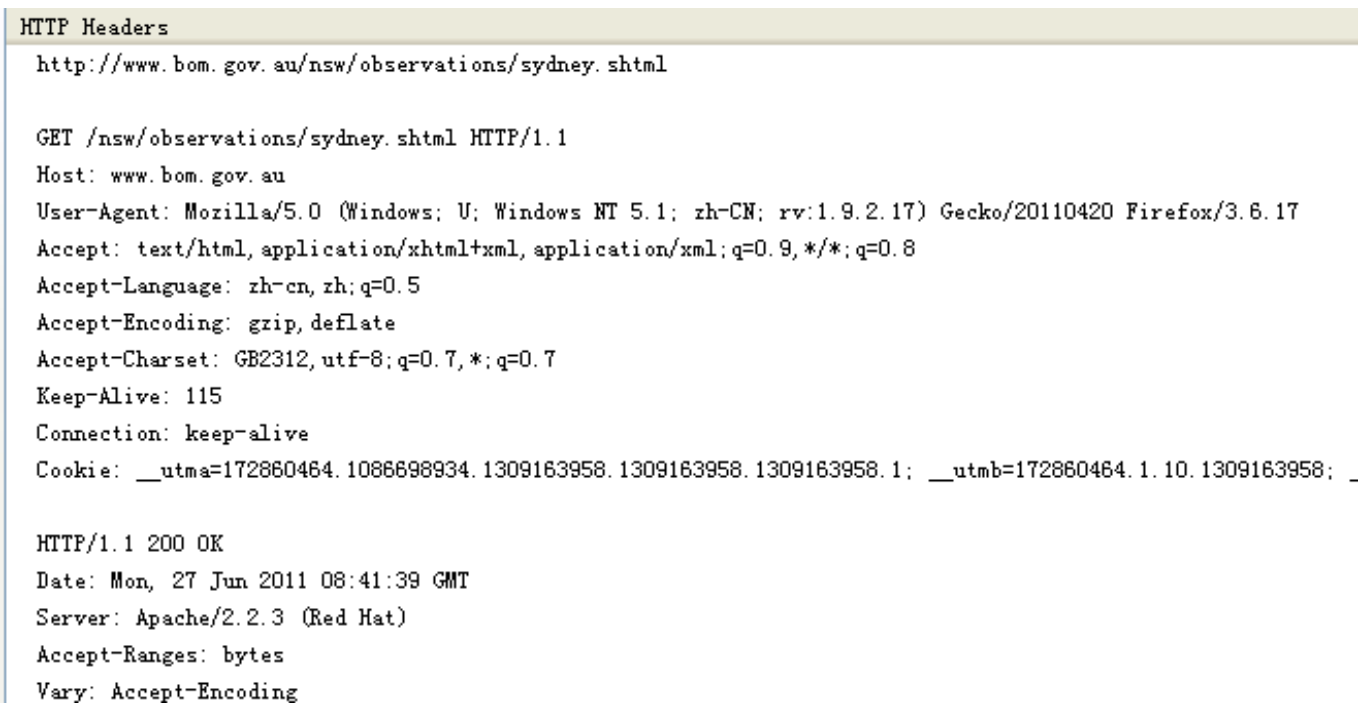

Figure 3.5: HTTP header of a request

Upon detection of a change in the webpage since the last visit, or upon first visiting this webpage, the parsing method will be triggered to extract useful information from the current page. When handling a large amount of data, the cost of system resource and bandwidth will grow, therefore the detecting frequency needs to be controlled at 
an acceptable level. In this case, the request will be sent every 1 minute to detect whether the webpage has been updated. The frequency is adjustable and ranges from every 3 seconds to 5 minutes.

Information embedded in the webpage is easy for humans to recognise but difficult for computers to read and understand. In this situation, Web scraping is used to extract data and structure machine readable information. Related techniques include text processing, semantic understanding, or regular expression matching (Schrenk, 2007).

Generally, Web scraping resolves a webpage into a Document Object Model (DOM), which views an HTML document as a tree structure. Tags and elements in the initial document are stored in the nodes of the DOM tree, and all the nodes in the tree can be accessed. To parse the DOM tree means to split it up based on some kind of delimiter. In order to parse such text efficiently, an open source library, "htmlcxx" (SourceForge, 2010) was incorporated into SIEVE-VTS. It can easily parse online content into desirable information and is able to precisely reproduce the original document from the parsed tree character by character. Using htmlcxx, segments of the webpage are captured as independent pieces of information. All the nodes are traversed using regular expression matching, and finally useless tags are removed and useful information is extracted (Figure 3.6).

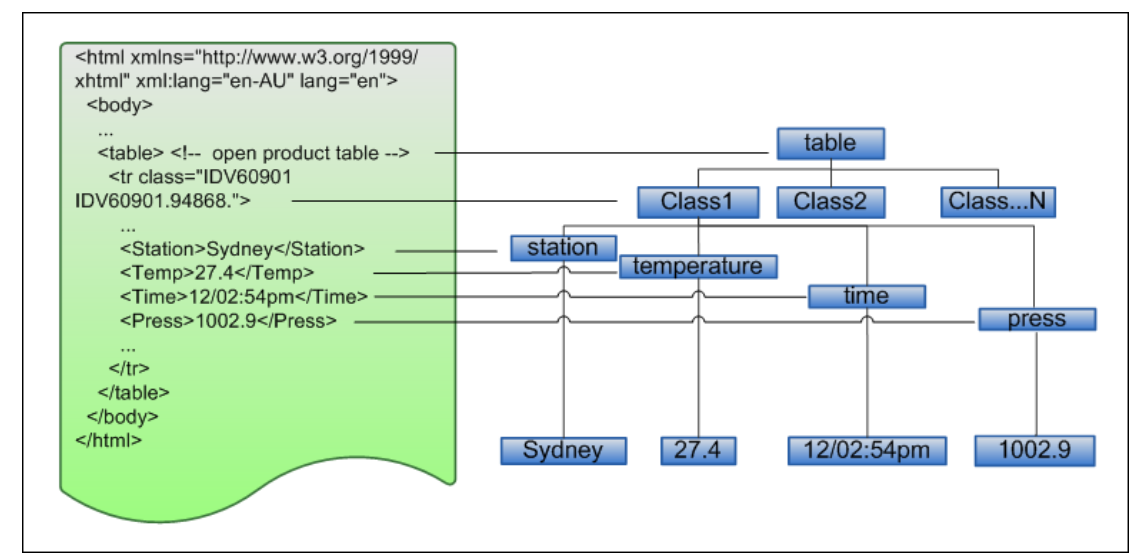

Figure 3.6: Parsing real-time weather condition

In the final step, the filtered data from the original web content is transferred and 
stored into MySQL (Figure 3.7). As discussed in Section 3.4, MySQL offers advanced features in rapid query and retrieval of data, as well as strong manipulation of spatial information. In order to interact with $M y S Q L$, a special module named DBManipulator in SIEVE-VTS was designed to facilitate database handling, including connecting to and disconnecting from the database, and executing SQL statements, such as insert, delete, update and query. Backing up of historical data can benefit future uses such as looking up past events, or re-exercise using data from previous times and locations.

\begin{tabular}{|c|c|c|c|c|c|c|c|c|c|c|c|c|}
\hline station & time & tmp & apptmp & dewpoint & relhum & deltaT & winddir & windspdkmh & windgustkmh & windspdkts & windgustkts & presshpa \\
\hline Sydney · Observatory Hill & 17/09:25am & 25.6 & . & 8.5 & 33 & 9.2 & . & . & . & . & . & 1022.4 \\
\hline Sydney Airport & 17/09:25am & 24.2 & 16.3 & 7.7 & 34 & 8.7 & $N$ & 39 & 48 & 21 & 26 & 1022.3 \\
\hline Sydney Harbour & 17/09:25am & 26.0 & 25.4 & 13.5 & 46 & 7.3 & $N$ & 9 & 13 & 5 & 7 & 1021.2 \\
\hline Sydney Olympic Park & 17/09:25am & 26.0 & . & . & . & . & $N$ & 13 & 17 & 7 & 9 & . \\
\hline Badgerys Creek & 17/09:25am & 23.9 & 23.4 & 13.7 & 53 & 6.0 & NNW & 9 & 9 & 5 & 5 & 1023.2 \\
\hline Bankstown & 17/09:25am & 24.7 & 20.4 & 10.9 & 42 & 7.7 & $N$ & 24 & 33 & 13 & 18 & 1022.3 \\
\hline Bellambi & 17/09:25am & 24.5 & . & . & . & . & $N$ & 30 & 32 & 16 & 17 & . \\
\hline Canden & 17/09:25am & 24.2 & . & . & . & . & $N$ & 9 & 11 & 5 & 6 & . \\
\hline
\end{tabular}

Figure 3.7: Real-time weather in $M y S Q L$

Web scraping is also used for collecting oceanographic information. Tide and swell data assists marine navigation and routing for crews on board, and ocean current data is useful for emergency responders when dealing with toxic incidents at the sea surface. Some public websites providing oceanographic information were investigated, such as willyweather (Willy Online Pty Ltd, 2010), NASA and BOM. Many datasets are available on these sites, such as temperature, waves, salinity and sea level. In this research, real-time tide and swell data was selected due to its accessibility and relevance for visualisation.

Tide is the rise and fall of sea levels, as a result of the gravitational forces exerted by the moon, the sun and the rotation of the earth. Tides have significant effects on the oceanic environment and the knowledge of tides is useful for offshore vessel navigation. It is interpolated by online content providers using tidal forecasts provided by the $B O M$ and Australian National Tide Tables. Data is provided four times a day, usually with 2 high tides and 2 low tides (Figure 3.8). 
Swell refers to the formation of long-wavelength surface waves, which are far more stable in direction and frequency than normal wind waves. Swell heights refer to the average of the highest one-third of swell waves. Swell information is presented in both tabular and graphical formats by online content providers and is updated twice a day (Figure 3.9).

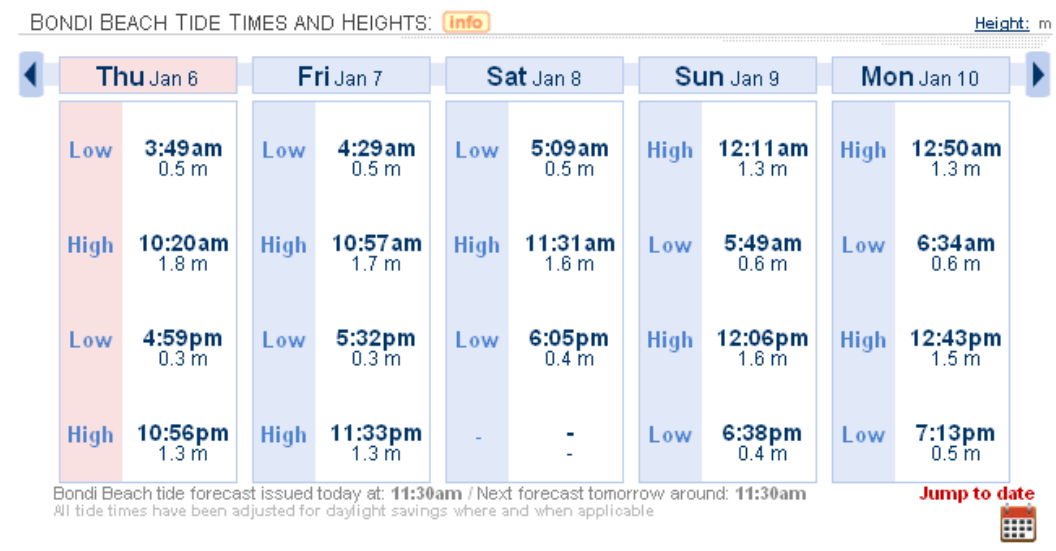

Figure 3.8: Oceanographic information in online service: tide time and heights

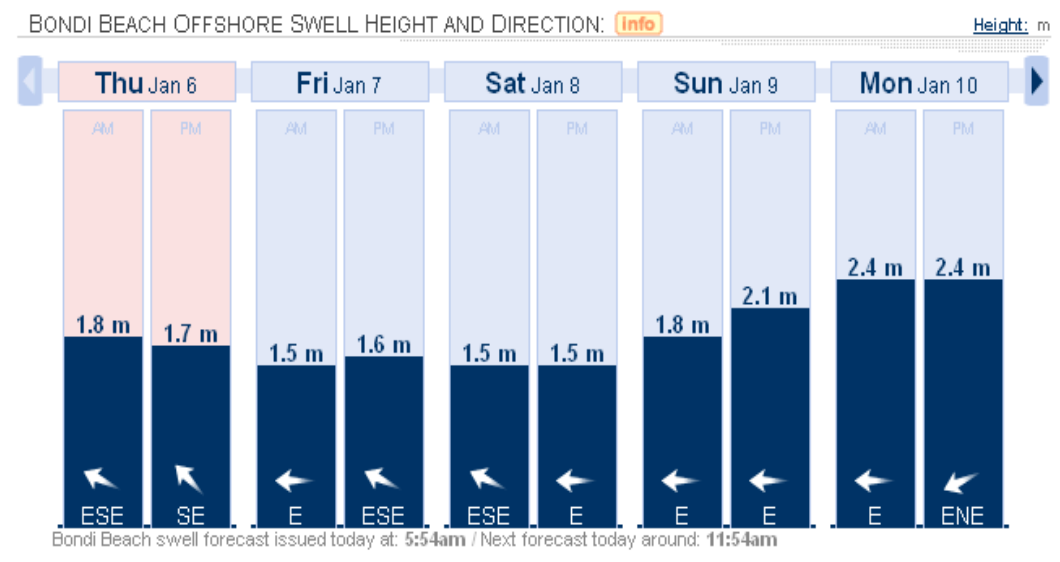

Figure 3.9: Oceanographic information in online service: swell height and direction

The technique for collecting real-time oceanographic data is similar to that used for real-time weather data. By sending a request for the latest tide and swell data of Sydney Harbour and receiving the HTTP header, the data spy can decide whether to download new pages or to use the old data. Valuable information can be extracted from the webpage: for tide data, time and sea height of high tide and low tide is 
recorded; for swell data, direction, height and frequency of the swell is obtained from the website by Web scraping (Figure 3.10). Finally, the data is stored into MySQL by DBManipulator (Figure 3.11).

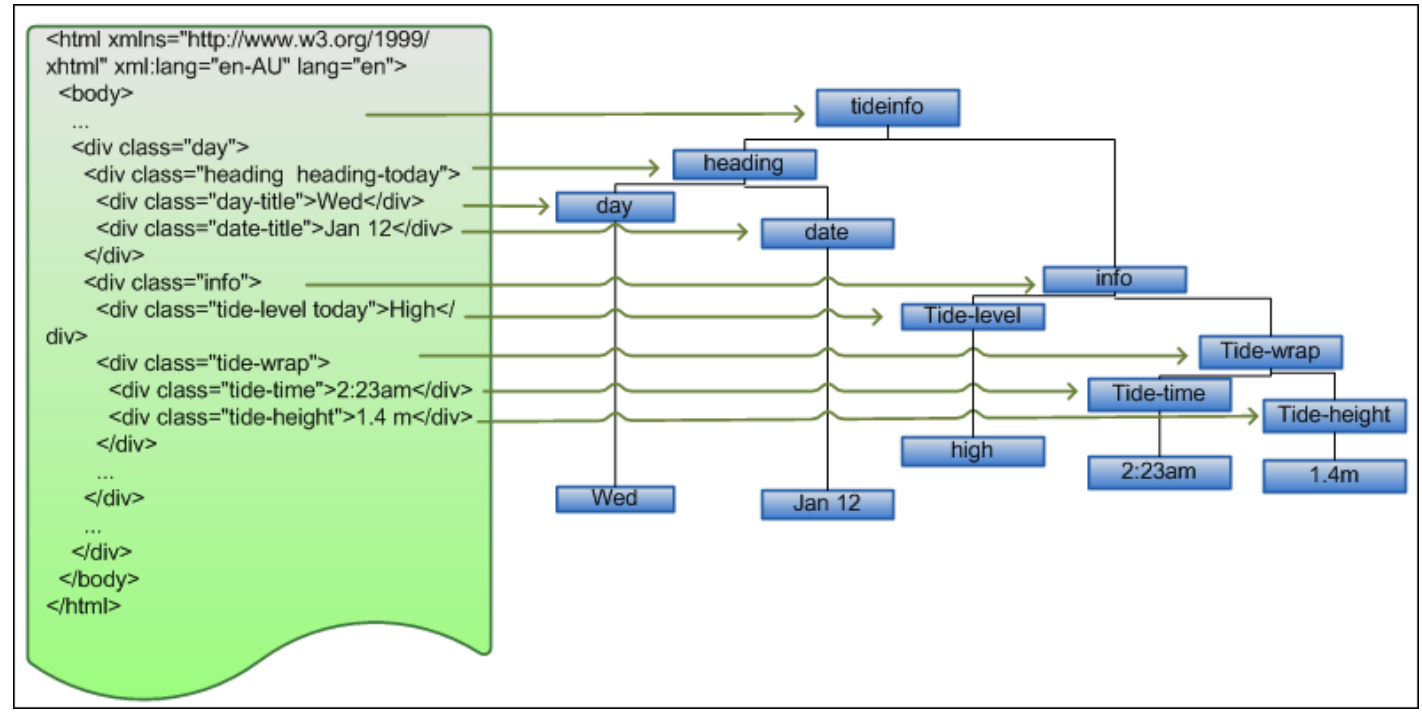

Figure 3.10: Parsing tide information into DOM

\begin{tabular}{|l|l|l|l|l|l|l|}
\hline \hline location & tideDate & tideTime & tideHeight & tideLevel & ID & station \\
\hline Sydney & Feb 1 & $22: 57: 00$ & $0.23 \mathrm{~m}$ & Low & 24 & Woolloomooloo \\
\hline Sydney & Feb 1 & $22: 59: 55$ & $0.88 \mathrm{~m}$ & High & 25 & Woolloomooloo \\
\hline Sydney & Feb 1 & $23: 01: 20$ & $0.25 \mathrm{~m}$ & Low & 26 & Woolloomooloo \\
\hline Sydney & Feb 1 & $23: 03: 44$ & $0.99 \mathrm{~m}$ & High & 27 & Woolloomooloo \\
\hline Sydney & Feb 12 & $20: 58: 50$ & $0.32 \mathrm{~m}$ & Low & 28 & Woolloomooloo \\
\hline Sydney & Feb 12 & $21: 01: 55$ & $0.98 \mathrm{~m}$ & High & 29 & Woolloomooloo \\
\hline Sydney & Feb 12 & $21: 02: 35$ & $0.25 \mathrm{~m}$ & Low & 30 & Woolloomooloo \\
\hline Sydney & Feb 12 & $21: 04: 50$ & $0.89 \mathrm{~m}$ & High & 31 & Woolloomooloo \\
\hline
\end{tabular}

Figure 3.11: Tide information in $M y S Q L$

There are some issues to be considered when collecting real-time data using Web scraping:

1. Legal issues sometimes occur when using Web scraping to obtain needed information. It is important to select appropriate websites for data collection and comply with the relevant copyright and confidentiality clauses.

2. When collecting large amounts of data from multiple sources, the webpage URL 
may be changed or the webpage structure may be modified. Therefore alternative methods may need to be utilised, such as mashup technology (Section 3.5.2).

\subsubsection{Collecting real-time data using mashup technology}

In Web scraping, users need to find the information of interest and scrape it from websites by themselves, which is an active collection method. A passive collection method is also applied in this research, such that users are notified automatically when new data is available online. An increasing number of content distributors publish their information by web feed, such as RSS or ATOM, which allows users to subscribe, not only for simple browsing, but also for integration into other applications. As was previously explained in Section 2.6.2, mashup technology uses APIs and third party data sources to provide new Web services.

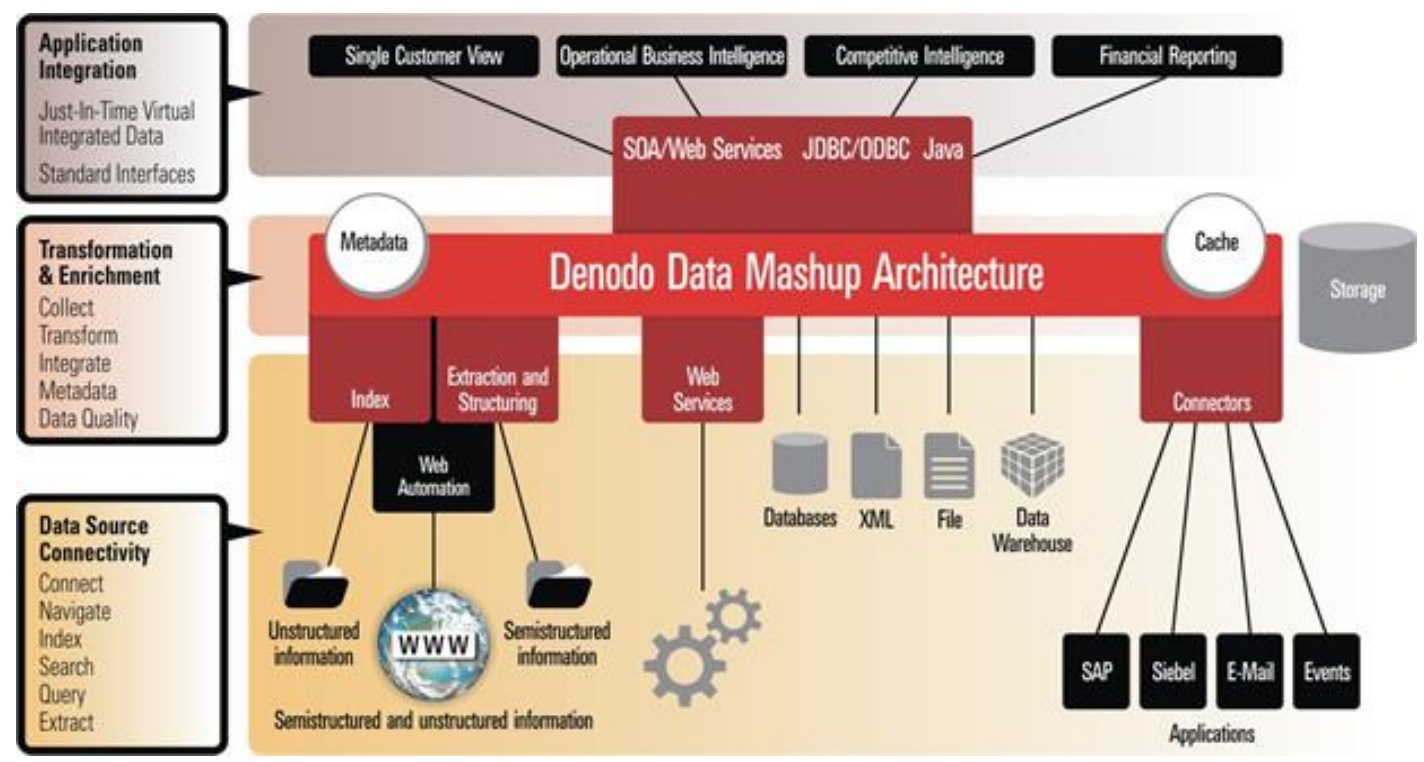

Figure 3.12: Mashup technique architecture (Gladstone USA, 2010)

SIEVE-VTS adopts a three-layer-architecture of mashup (Figure 3.12), aiming to collect the latest web feeds and visualise the results in real time:

Data layer deals with data manipulation including sending, storage and retrieval. Data is transferred in RSS format, which is essentially a standardised XML document containing full text and metadata. XML is a common format for data distributors 
publishing frequently updated information, it is also machine readable and can be easily integrated into other applications.

Web service layer provides APIs for clients to retrieve the data feeds. XMLHTTPRequest, a third party API that executes client side functions to transfer data between a client and a server, was integrated in SIEVE-VTS. It is used for sending requests and receiving RSS data.

Presentation layer defines the user interface and is usually implemented in web browsers by traditional mashup techniques. In this research, RTDSimulator, another module of SIEVE-VTS, supports visualising the presentation results in the virtual environment.

Mashup is used for collecting Automatic Identification System (AIS) information. AIS, an automated tracking system installed on a vessel, transfers and exchanges maritime information with nearby ships or Vessel Traffic Services (VTS) stations (Figure 3.13). An AIS electronic transponder can continuously broadcast information obtained from GPS and gyrocompass mounted on the ship, including the vessel's position, speed and course. AIS receivers fitted on other crafts or VTS can receive the information then calculate and display it on a screen or chart plotter.

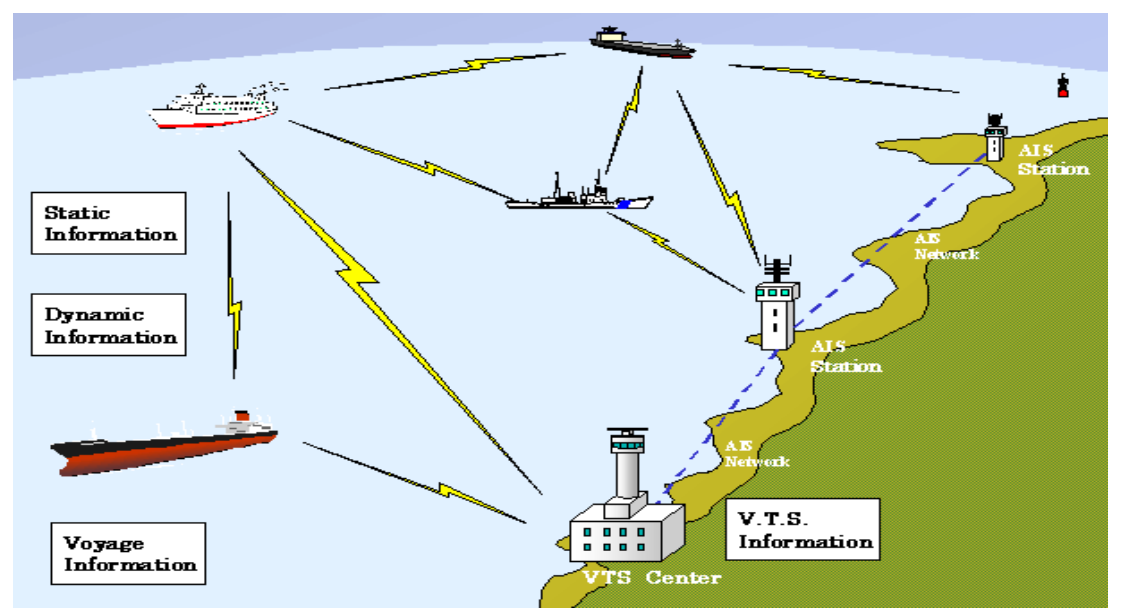

Figure 3.13: AIS schematic diagram

AIS data is collected for emergency management as it plays an invaluable role in 
helping people to enhance maritime security, such as providing electronic navigation aids to vessels, avoiding the risk of collisions in busy traffic areas, searching and rescuing vessels that encounter danger and investigating reasons for marine accidents.

Some online content providers offer compiled AIS data by web feeds. Representative examples are "MarineTraffic" (University of the Aegean, 2010), "VesselTracker" (Vesseltracker, 2010), "AIS Hub" (Astra Paging Ltd, 2010). AIS data is collected by the receivers installed on board, and wirelessly sent to a remote central database. The central database receives and processes mass data from numerous vessels including port and area geographic information, vessels' real-time location and other information. The current position and tracks of vessels can be displayed using the Google Maps API (Figure 3.14). Position history, vessel's details, and port information are also stored in the database.

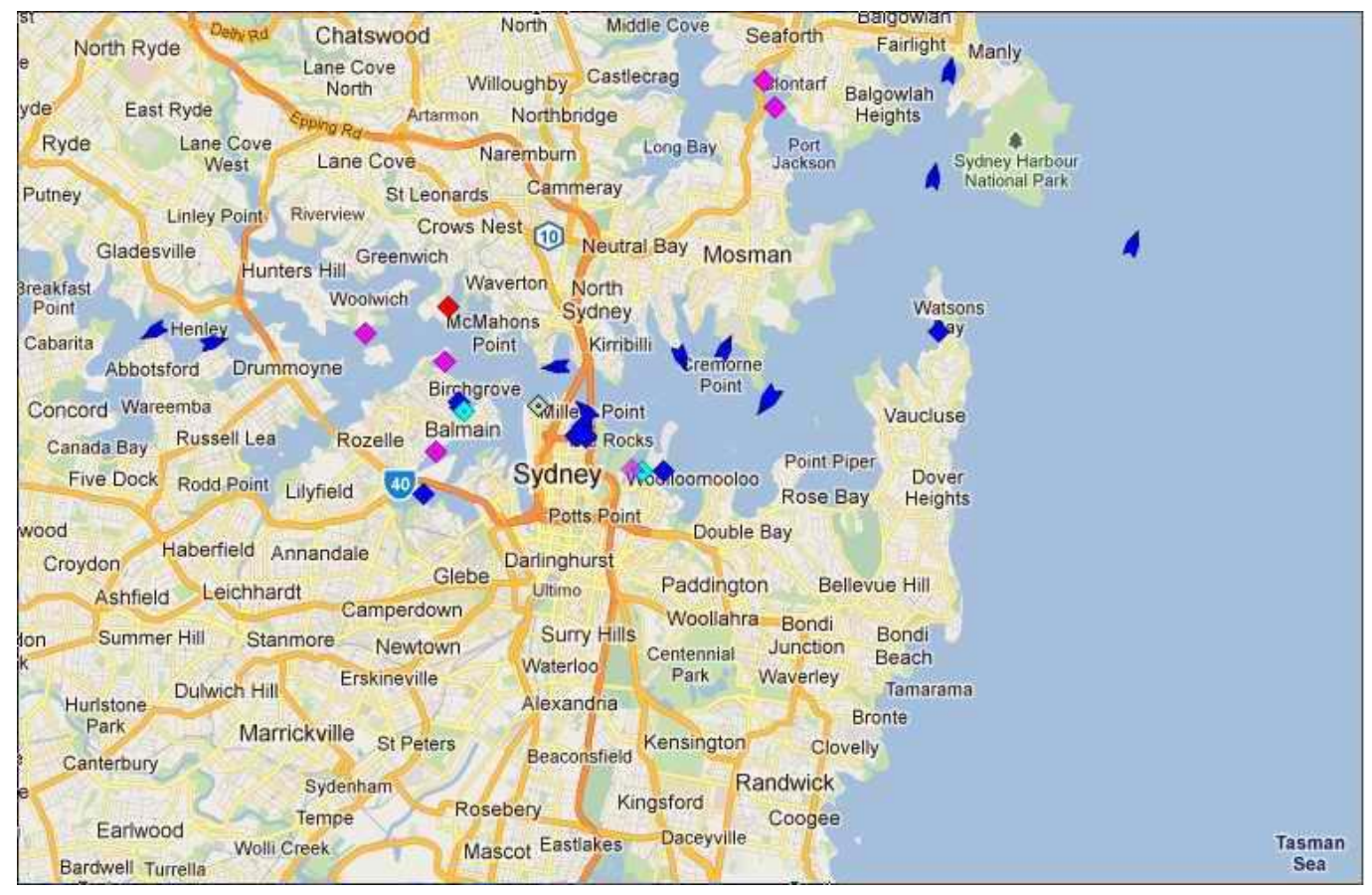

Figure 3.14: Online AIS data (University of the Aegean, 2010)

The XMLHTTPRequest functions are used to send requests to servers for RSS. The "GET" method is called to request data from specified sources (Figure 3.15). Connections are kept alive between the client and server, thus less time is spent on 
requesting new connections. When new data is updated on the server, it will push RSS into the clients' aggregator that can be scheduled to check for new content periodically, in this case, every 6 seconds. After the server receives the request and validates it, a list of AIS information, formatted in XML, is returned to the client (Figure 3.16).

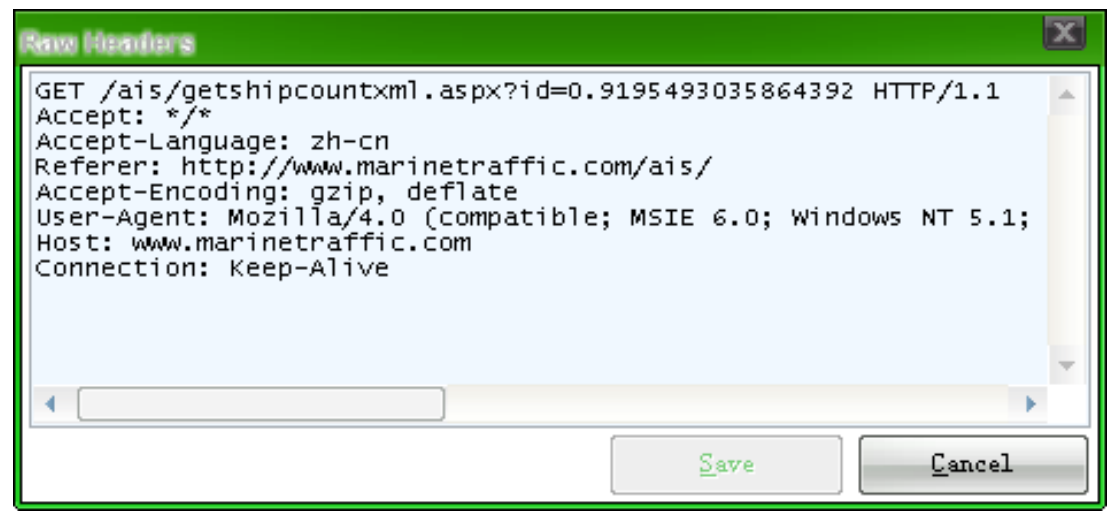

Figure 3.15: Request data from servers

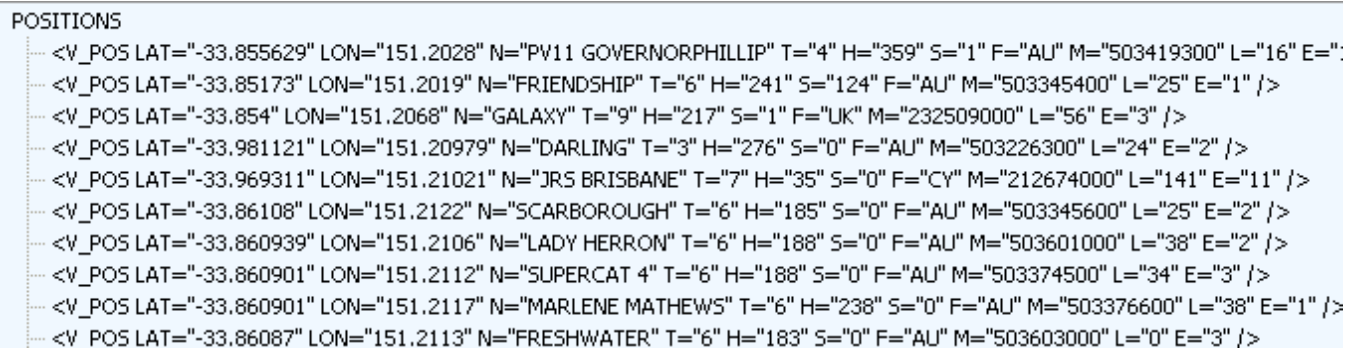

Figure 3.16: AIS information in an XML file

Information in the AIS data sheet above includes: vessel's name (N), MMSI (M) or the identification number, type $(\mathrm{T})$, length $(\mathrm{L})$, country flag $(\mathrm{F})$, current position using latitude and longitude (LAT and LON), heading direction or course $(\mathrm{H})$, current speed (S) and time since the current data was received (E). By implementing "TinyXML" library in SIEVE-VTS, the XML document can be easily parsed into DOM, which can be read, modified, and saved. By traversing the elements in the DOM tree from root nodes to leaf nodes, useful information can be parsed and stored into MySQL (Figure 3.17). 


\begin{tabular}{|c|c|c|c|c|c|c|}
\hline $\mathrm{b}$ Id & Time & longitude & lat & mmsi & speed & course \\
\hline 2765 & $2009-02-0100: 36: 51$ & 151.2194519 & .33 .8557587 & 235739000 & 12.300 & 109.700 \\
\hline 2774 & 2009-02-01 00:37:01 & 151.2201233 & -33.8558922 & 235739000 & 12.000 & 100.600 \\
\hline 2782 & $2009-02-0100: 37: 08$ & 151.2205963 & .33 .8559380 & 235739000 & 12.100 & 94.200 \\
\hline 2783 & 2009-02-01 00:37:10 & 151.2207184 & -33.8559418 & 235739000 & 11.800 & 91.800 \\
\hline 2789 & $2009-02-0100: 37: 14$ & 151.2209778 & .33 .8559303 & 235739000 & 11.500 & 91.400 \\
\hline 2793 & $2009-02-0100: 37: 18$ & 151.2212219 & -33.8559341 & 235739000 & 11.100 & 89.900 \\
\hline 2797 & $2009-02-01$ 00:37:22 & 151.2214203 & .33 .8559341 & 235739000 & 10.800 & 90.600 \\
\hline 2801 & $2009-02-01$ 00:37:24 & 151.2215881 & -33.8559380 & 235739000 & 10.700 & 89.800 \\
\hline 2803 & $2009-02-01$ 00:37:28 & 151.2218170 & -33.8559265 & 235739000 & 10.400 & 87.600 \\
\hline 2806 & $2009-02-01$ 00:37:31 & 151.2219849 & .33 .8559151 & 235739000 & 10.400 & 86.700 \\
\hline 2810 & $2009-02-0100: 37: 35$ & 151.2222138 & -33.8559074 & 235739000 & 9.900 & 88.500 \\
\hline 2815 & $2009-02-0100: 37: 40$ & 151.2224731 & -33.8558922 & 235739000 & 9.600 & 88.300 \\
\hline
\end{tabular}

Figure 3.17: AIS data in $M y S Q L$

In addition to the primary weather information from $B O M$, some auxiliary weather data can be collected from Yahoo! Weather, which provides RSS feeds containing the latest weather conditions, including visibility, astronomy information and basic weather conditions. Such information can supplement the primary weather data and is more suitable for visualising in the virtual environment.

One example of sending requests to collect the latest RSS feeds from Yahoo! Weather can be structured as: "http://weather.yahooapis.com/forecastrss? $\mathrm{w}=1105779 \& \mathrm{u}=\mathrm{c}$ ". The URL is followed by parameters and values with a question mark. The " $w$ " refers to WOEID, a unique reference identifier assigned by Yahoo! to identify any location on the earth. The " $u$ " refers to the degree units of weather information and the value can be either Fahrenheit (f) or Celsius (c).

After receiving the request, the server will return a weather RSS feed responding to the request. Latest weather information is transferred in XML format (Figure 3.18). It contains weather information such as wind speed and direction, atmosphere pressure and temperature, some of which duplicates information provided by BOM. Therefore, only basic weather condition, visibility, and time of sunrise and sunset are selected as complementary weather information. 


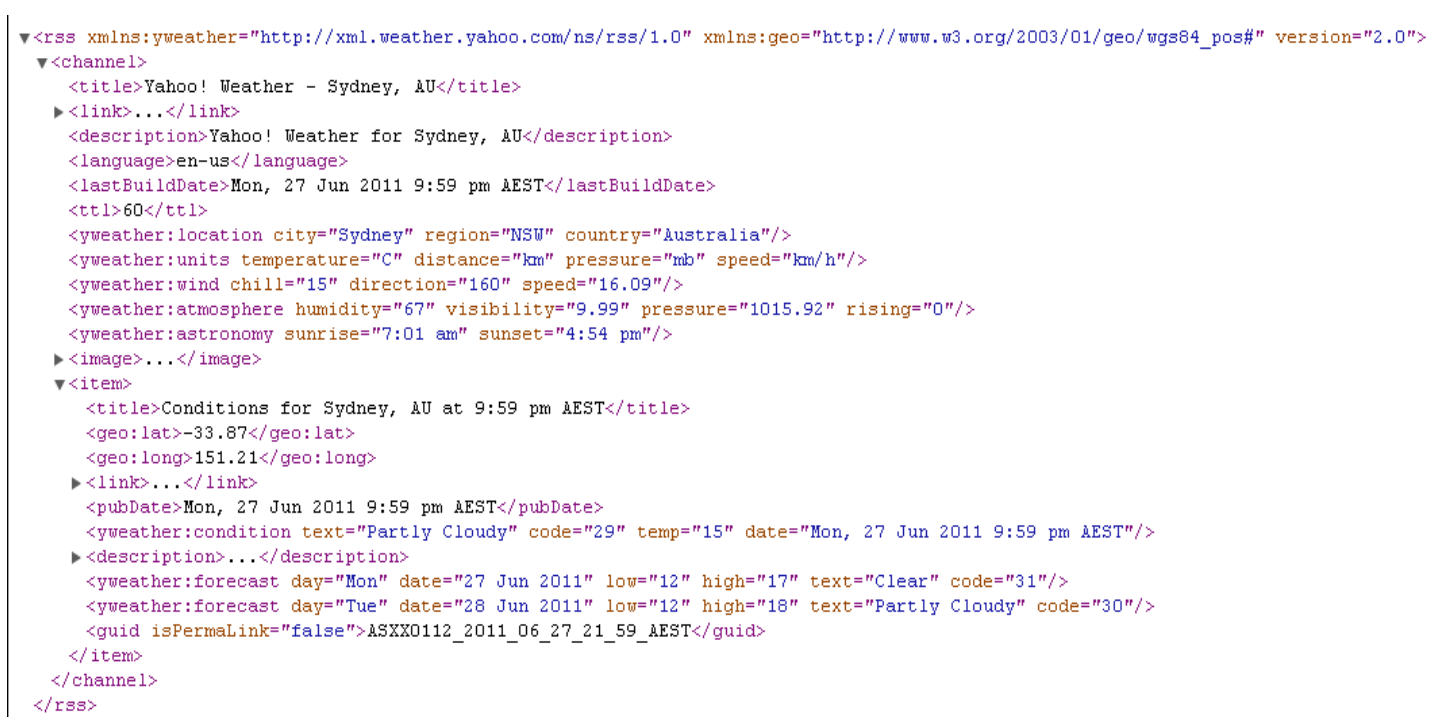

Figure 3.18: Weather in RSS from Yahoo! Weather

Basic weather conditions are described in both text and code. For example, a literal weather condition can be described as "mostly cloudy" or "scattered showers" and stored as string format. Weather condition code is used to represent 48 kinds of weather conditions defined in Yahoo! Weather (Appendix 1). Visibility is specified as the visual distance, whereby a visibility of 20.5 kilometres will be displayed as "2050". The units are determined by the distance attribute of the "yweather:units" element, either in "mi" or "km". Sunrise and sunset times are represented as a string in a local time format, e.g. "6:05 am". Parsing strategy is similar to that applied to AIS information. Finally, the auxiliary weather information is extracted and stored in MySQL (Figure 3.19).

\begin{tabular}{|c|c|c|c|c|c|c|c|c|c|}
\hline location_city & condition_code & condition_text & atmosphere_visibility & astronomy_sunrise & astronomy_sunset & geo_lat & geo_long & date_time & location_country \\
\hline Melbourne & 26 & cloudy & 5.21 & $5: 55 \mathrm{am}$ & $8: 19 \mathrm{pm}$ & -37.74 & 144.9 & $2009-02-1220: 58: 50$ & AS \\
\hline Melbourne & 11 & showers & 7.21 & $5: 55 \mathrm{am}$ & $8: 19 \mathrm{pm}$ & -37.74 & 144.9 & $2009-02-1502: 43: 55$ & AS \\
\hline Melbourne & 26 & cloudy & 3.21 & $5: 27 \mathrm{am}$ & $8: 03 \mathrm{pm}$ & .33 .96 & 151.01 & 2009-02-01 22:57:00 & AS \\
\hline Melbourne & 27 & Mostly Cloudy & 6.21 & 7:12 am & $5: 20 \mathrm{pm}$ & .37 .74 & 144.9 & Sat, 15 May $2010 \ldots$ & AS \\
\hline Melbourne & 27 & Mostly Cloudy & 6.21 & $7: 12 \mathrm{am}$ & $5: 20 \mathrm{pm}$ & -37.74 & 144.9 & Sat, 15 May $2010 \ldots$ & AS \\
\hline Melbourne & 27 & Mostly Cloudy & 6.21 & 7:12 am & $5: 20 \mathrm{pm}$ & .37 .74 & 144.9 & Sat, 15 May $2010 \ldots$ & AS \\
\hline Sydney & 33 & Fair & 6.21 & $5: 55$ am & $8: 19 \mathrm{pm}$ & -37.74 & 144.9 & $2009-02-1220: 58: 50$ & AS \\
\hline Sydney & 4 & thunderstor... & 12.26 & $5: 10 \mathrm{am}$ & $7: 50 \mathrm{pm}$ & 34.05 & -118.23 & 2009-02-01 22:57:00 & AS \\
\hline Sydney & 26 & cloudy & 7.21 & $5: 27 \mathrm{am}$ & $8: 03 \mathrm{pm}$ & .33 .96 & 151.01 & $2009-02-1502: 43 \cdot 55$ & AS \\
\hline
\end{tabular}

Figure 3.19: Auxiliary weather information in $M y S Q L$

\subsubsection{Collecting real-time data using mobile device}

Information collected onsite is commonly made available through web applications, 
which can be provided in real time to public or private sectors. For example, the online fire incident summary provided by Victoria's Country Fire Authority (CFA) displays up-to-date information regarding fire locations and status to the public (Country Fire Authority, 2010). Information including address, date, time, incident type, status, size and appliance in attendance is tabulated and visualised in Google Maps (Figure 3.20).

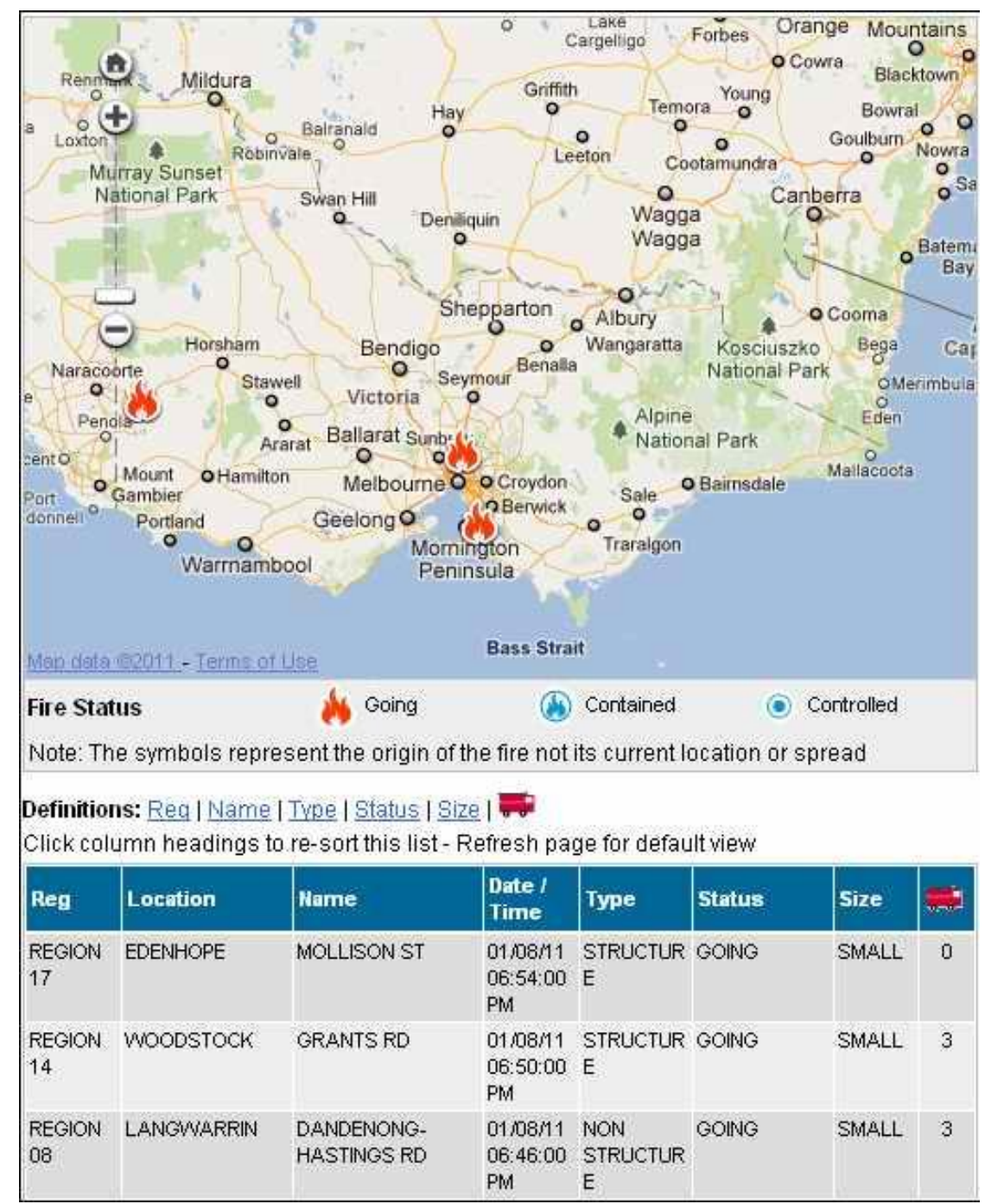

Figure 3.20: CFA incident summary website (Country Fire Authority, 2010)

Rather than integrating real-time data from other online content providers, $C F A$ directly obtains firsthand fire incident information, which is created from the latest fire emergency phone calls or reports by those who witness or encounter incidents. Collecting onsite information can obtain original data, save time on secondary 
distribution and reduce loss of accuracy. It can also solve issues that arise when data is not available from the content providers, or there is copyright restriction on the data.

PDA, GPS devices and smart phones are ideal tools for obtaining real-time data from onsite locations. In this research, iPhone, a multimedia-enabled and Internet connected smart phone (Apple Pty Ltd, 2010), was selected as the primary device for collecting onsite information. It is appropriate for this task due to its advanced built-in system, including embedded GPS, digital compass, high resolution camera, and 3G cellular network capabilities.

iPhone has been utilised for real-time data collection in previous studies. An example of this was seen at IBM's Almaden Research Centre whose scientists developed a "Creek Watch" iPhone application that helped monitor debris and water quality of creeks (Kim, Robson, Zimmerman, Pierce, \& Haber, 2011). Additionally, the "openmap" application on iPhone (IZE Ltd, 2010) was designed for updating the map data of "OpenStreetMap" (OpenStreetMap, 2010), such as editing a street or place of interest.

In order to collect onsite data around Sydney Harbour and transmit it into SIEVE-VTS, a sub-system is designed that combines iPhone application development, XML packaging and parsing, as well as Java Servlet technology (Oracle, 2010b). The application is named iReport, which is based on UIKit, a high-level Objective-C framework that deals with the graphical front-end of the iPhone Operation System. The working flow of the system is designed as follows (Figure 3.21): when a user starts the application, the GUI is launched and displayed. After the user's interaction and input, the data is wrapped as an XML file which is easy for data transfer and sharing. The wrapped XML file is instantly transferred to a remote server by HTTP request via wireless connection. On the server side, a Java Servlet responds to the request and receives the uploaded data from iReport using the "doPost" method. Since the data is formatted in XML, reverse engineering of XML wrapping is applied. Similar to parsing the weather and AIS data, elements are extracted, tags are deleted, 
and the information embedded in the XML file is stored into $M y S Q L$.

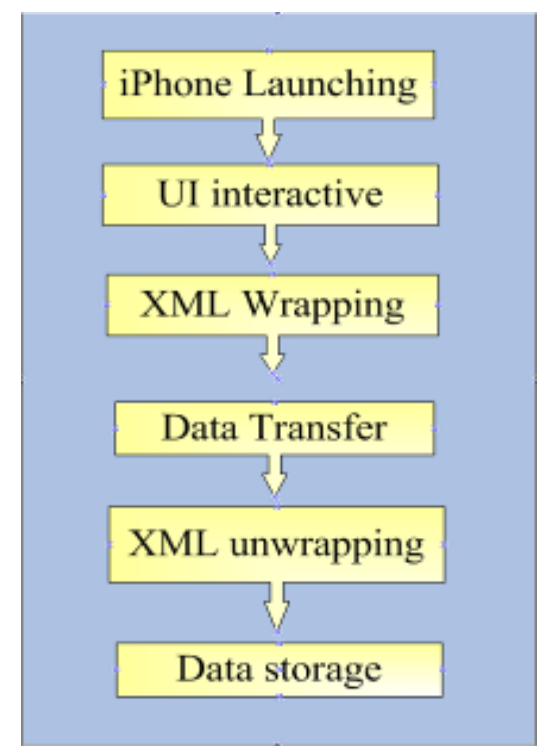

Figure 3.21: Operation flow of iReport

The application of iReport is mainly used for collecting real-time incident location and status (Figure 3.22). It contains incident type, scale and status, which provide basic information for emergency responders, and support planning for emergency management. When observing an incident in a real emergency situation, a safety patrol can use the iPhone and launch the iReport application. By tapping on the touch screen, the safety patrol can choose the type, status and size of the incident from the sub-menu in the "Location Info" category. Location information is automatically retrieved from the GPS and recorded in the program when "Current Location" is turned on. If there is a poor GPS signal, the longitude, latitude or address can be entered directly into the "Location Info" category by the user. The embedded camera can also be used to photograph an incident, with the compass recoding its orientation. After the safety patrol presses the "Submit Data" button, the information is sent to a remote server immediately. 


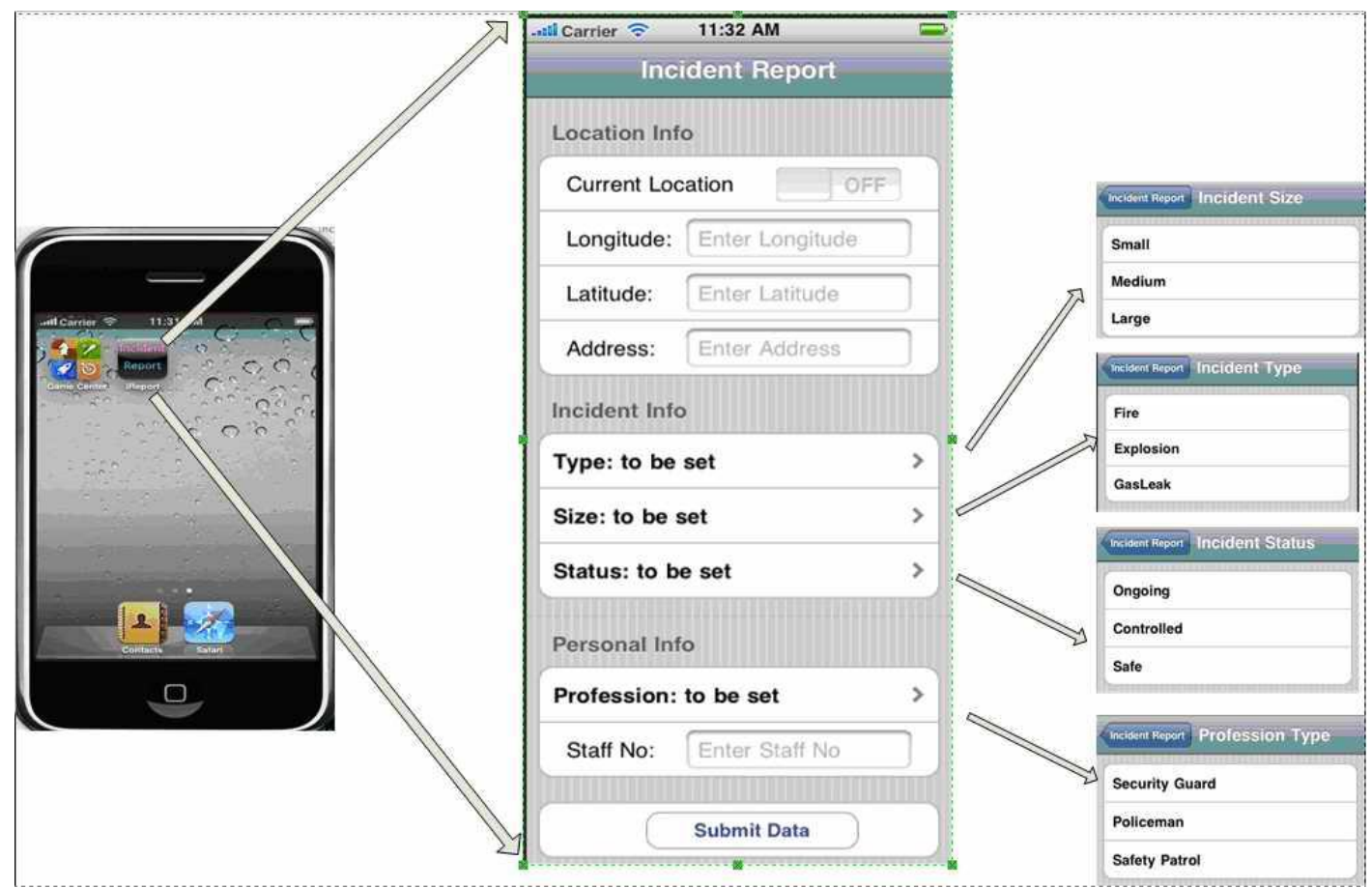

Figure 3.22: iReport user interface

\subsection{Real-Time Data Translation}

As detailed in the previous section, real-time data is obtained by RTDCollector and stored into the MySQL database. However, such data cannot be instantly visualised in SIEVE-VTS, due to it being abstracted information from the real world which quantitatively describes an event. At this point an event is defined as anything that takes place with spatial and temporal attributes, such as a fire incident occurring in Sydney Harbour, or a ship moving from one location to another. SIEVE-VTS is based on a game engine, which can efficiently simulate the real world in a virtual environment employing physics, lighting, sound, graphs, animation, and Artificial Intelligence (AI). Differences in the structures of the real and virtual environment require a translation between the abstract data from the real world and the simulated representation in the virtual environment. In this section, three fundamental translations are investigated: location translation, time translation, and attribute translation. 


\subsubsection{Location translation}

Real world spatial information is recorded as latitude and longitude in geodetic coordinates, which is a common way to describe the accurate position of an entity on earth. Location data in the CVE is represented in a Cartesian coordinate system, such that each point in a plane is represented by a pair of numeric coordinates (x, y). Therefore, location translation deals with the transformation between geodetic coordinates and Cartesian coordinates.

Converting spatial data is based on the assumption that the area is flat, and earth curvature is not sufficient to be taken into account. This is true if the original area is small, in this case, a $2.5 \mathrm{~km} \times 2.5 \mathrm{~km}$ area. Therefore a linear transformation can be utilised such that points are scaled equally in the $\mathrm{X}$ and $\mathrm{Y}$ dimensions, and shifted from one coordinate system to the other without rotation.

Often the transformation is known because it is defined by the developer of the virtual environments. However, there are cases in which it is necessary to compute the transformation using known locations in both environments. RTDTranslator performs the coordination transformation in the following steps:

1. Three control points are selected in the research field and recorded as $\left(L A T_{1}, L O N_{1}\right)$, $\left(L A T_{2}, L O N_{2}\right),\left(L A T_{3}, L O N_{3}\right)$, while the corresponding Cartesian coordinate points in the CVE are recorded as $\left(X_{1}, Y_{1}\right),\left(X_{2}, Y_{2}\right),\left(X_{3}, Y_{3}\right)$.

2. Use the 3-parameter Bursa-Wolf Model (Bursa, 1962; Wolf, 1963), and substitute the values of the 3 pairs of coordinates into the equation:

$$
\begin{aligned}
& L A T=A_{1} * X+B_{1} * Y+C_{1} \\
& L O N=A_{1} * X+B_{2} * Y+C_{2}
\end{aligned}
$$

3. Use the method of least squares to calculate the parameters. Finally the transformations between Cartesian coordinate in SIEVE-VTS and Geodetic 
coordinates in the real world can be represented as:

\begin{tabular}{|c|c|}
\hline$L A T=A_{1} * X+B_{1} * Y+C_{1 \ldots}$ & equation 3.1 \\
\hline$L O N=A_{2} * X+B_{2} * Y+C_{2 \ldots}$ & equation 3.2 \\
\hline$X=A_{1}^{\prime} * L A T+B_{1}^{\prime} * L O N+C_{1}^{\prime}$ & equation 3.3 \\
\hline$Y=A_{2}^{\prime} * L A T+B_{2}^{\prime} * L O N+C_{2}^{\prime}$. & equation 3.4 \\
\hline
\end{tabular}

indicating the forward and inverse transformation respectively.

From the equations it can be seen that location translation is bi-directional. When an event occurs in the real world, the corresponding location in SIEVE-VTS is calculated (equation 3.1, equation 3.2). In contrast, if an event happens at a certain location in the CVE, the real location in latitude and longitude can be calculated and displayed to the end users (equation 3.3, equation 3.4).

\subsubsection{Time translation}

Time in SIEVE-VTS identifies the commencement, continuance and termination of an event. Time translation is implemented based on the different time measurements in the real world and the virtual environment.

Time initialising:

When a scenario is loaded in SIEVE-VTS, the default time setting is "00:00:00", indicating initialisation of a new scenario. However, collecting and simulating real-time data is based on universal time in the real world. In order to synchronise the real world time with the time in the virtual environment, a modified function "GetLocalTime()" is called to retrieve the current date and time, and the returned value is bound with the starting time of the scenario.

Time lapsing:

1) Synchronous time: this is the default mode of time lapsing in the virtual 
environment. In such circumstances, the event starts and ends at the same time in the virtual world as in the real world. Specifically, it is utilised when streaming real-time data by RTDCollector and visualising the results using RTDSimulator.

2) Asynchronous time: this is an alternative mode used when time needs to run faster or slower in the virtual world than in the real world. This is achieved by adapting a transform equation:

$C_{t}(t)=t_{0}+k * t$ equation3.5

where $C_{t}(t)$ is the current time in the virtual world, $t_{0}$ is the initialising time, $k$ is an extension or contraction factor assigned by users, and $t$ is the time lapsing in the real world. This function is useful for visualising historical data for evaluation or slow-motion replaying of rapid incident sequences.

In SIEVE-VTS, time is assigned as an attribute with an event using the object-oriented method, and events are recorded in the database according to their time series. When time passes a certain point, corresponding events are triggered and simulated in the virtual environment.

\subsubsection{Attribute translation}

In SIEVE-VTS, or specifically in the RTDSimulator, real-time information such as weather or AIS, is modelled as a multi-media scene composed of frames of graphics, video, audio and text. The attribute translation deals with converting the original data format to visual results. Table 3.3 shows some of the conversions from original data to SIEVE-VTS supported models.

Among the weather elements, wind speed and direction can be represented by the movement of floating objects, such as cloud, leaves, and dust in the air. Sunrise and sunset time is modelled using the variation of atmosphere brightness with time. 
Precipitation can be modelled using different types and amount of particles in the virtual world. Visibility can be modelled into fog-level in the game engine. Basic weather conditions can be designed using several basic datablocks of sky with different texture and precipitation types.

Converting oceanographic data firstly involves the changes of tide height with time, which is modelled as a sinusoidal function. The vertical movement of a swell is translated as another sinusoidal function with a lower amplitude and higher frequency than the tides. The horizontal movement of swell can be modelled as the wave movement of the sea surface.

For converting AIS information, designing 3D models to represent the shape of a vessel is an appropriate method to translate attributes about ships such as "ship type", "length", and "breadth". Traffic information, such as speed and course of vessels, can be translated into movement models of multiple vessels incorporating AIS data.

When dealing with incident location and status, different types of incidents are simulated by different scene models, such as explosion, fire, or gas leak, which are rendered in real time in SIEVE-VTS. Size of the simulated incident is determined by the incident area and object scale. Incident status is simulated by the change of incident conditions with time. 


\begin{tabular}{|c|c|c|}
\hline Category & $\begin{array}{l}\text { Real-time data } \\
\text { key parameters }\end{array}$ & $\begin{array}{l}\text { Visualisation result } \\
\text { key parameters }\end{array}$ \\
\hline \multirow{7}{*}{ Weather Condition } & Time $<$ Date $><$ Hour $><$ Minute $>$ & Synchronised time \\
\hline & Wind $<$ Direction $><$ Speed $>$ & $\langle$ cloudSpeed $>,<$ windVelocity $>$ \\
\hline & Astronomy $<$ Sunrise $><$ Sunset $>$ & $\langle$ Sky_Mode $><$ Sun_Mode $>$ \\
\hline & Precipitation $<$ type $><$ amount $>$ & $\langle$ Drop_Type $><$ Drop_Size $<$ Drop_Intensity $>$ \\
\hline & Visibility<distance $>$ & Visibility<virtual_distance> \\
\hline & Condition code $(0,1, \ldots 48)$ & Datablock based weather condition \\
\hline & Temperature, relative humidity, etc & GUI \\
\hline \multirow{4}{*}{$\begin{array}{l}\text { Oceanographic } \\
\text { Information }\end{array}$} & Time $<$ Date $><$ Hour $><$ Minute $>$ & Synchronised time \\
\hline & Tide & Tidal constituents model \\
\hline & Swell<Horizontal $>$ & Sea surface movement \\
\hline & Swell<Vertical $>$ & Swell model \\
\hline \multirow{5}{*}{ AIS Information } & Time $<$ Date $><$ Hour $><$ Minute $>$ & Synchronised time \\
\hline & Location $<$ Log $><$ Lat $>$ & Cartesian coordinates in CVE \\
\hline & Speed and course & Traffic model \\
\hline & Vessel type and shape & 3D static models \\
\hline & Other information & GUI \\
\hline \multirow{5}{*}{$\begin{array}{l}\text { Incident Location and } \\
\text { Status }\end{array}$} & Time $<$ Date $><$ Hour $><$ Minute $>$ & Synchronised time \\
\hline & Location $<$ Log $><$ Lat $>$ & Cartesian coordinates in CVE \\
\hline & Type<Fire | Explosion | Gas leak> & Type model \\
\hline & Size & Incident area and object scale \\
\hline & Status $<$ Heavy $\mid$ Medium $\mid$ Light $>$ & Change of conditions \\
\hline
\end{tabular}

Table 3.3: Attribute translation in SIEVE-VTS

\subsection{Real-Time Data Simulation}

Real-time data is visualised and simulated in SIEVE-VTS via the RTDSimulator module. This module relies on Torque Game Engine Advanced, which has some intrinsic features providing foundations for the simulation.

The first feature is the graphics system which is a fully hardware abstracted rendering system. Based on that, utilisation of lighting and shadow can support the environmental simulation. Another feature is an integrated particle engine which supports texture and scale animation and is especially useful when simulating dynamic scenarios, such as precipitation and fog in weather condition, or explosion 
and fire in incident scenarios. The third feature is that it uses a physical engine controlling basic physics of objects in the virtual environment, such as collision detection, rigid body, and vehicle physics. This function potentially supports the physical effects associated with environmental elements, such as the damage to buildings and characters caused by explosion, and the collision of ships and vehicles.

With the support of such features, RTDSimulator is designed to simulate the scenario integrating real-time data. In the following sections, the simulation of each type of data is illustrated in detail.

\subsubsection{Weather simulation}

A simulation system inside a game engine is very realistic and can affect the way in which users perceive and behave in the virtual environment, including their vision, movement and decision making. The common trend amongst simulation systems is that they get as close to real life as possible, and by doing so, they model familiar scenes and objects that users can refer to, and increase the overall authenticity of the user experience (Meyer, 2006). They are therefore more likely to behave in a similar way in the virtual environment as in the real world. With this as a key consideration, weather condition is divided into several major elements which can be simulated respectively. These elements include: precipitation, sunlight, wind, visibility, and basic weather condition.

Weather elements are encapsulated in a datablock, which is a special data type used to define objects in TGEA. The variables in the datablock are initialised by default values, and when real-time data is updated in the database, the corresponding datablock variables will be automatically changed and the related visualisation procedures will be triggered.

Precipitation simulates any product of the condensation of atmospheric water vapour that falls onto the ground, usually in the form of rain, snow or hail. In RTDSimulator, 
precipitation is encapsulated into "Precipitation" and "PrecipitationData" datablocks. Code Extract 3.1 demonstrates how this is achieved by highlighting important parameters.
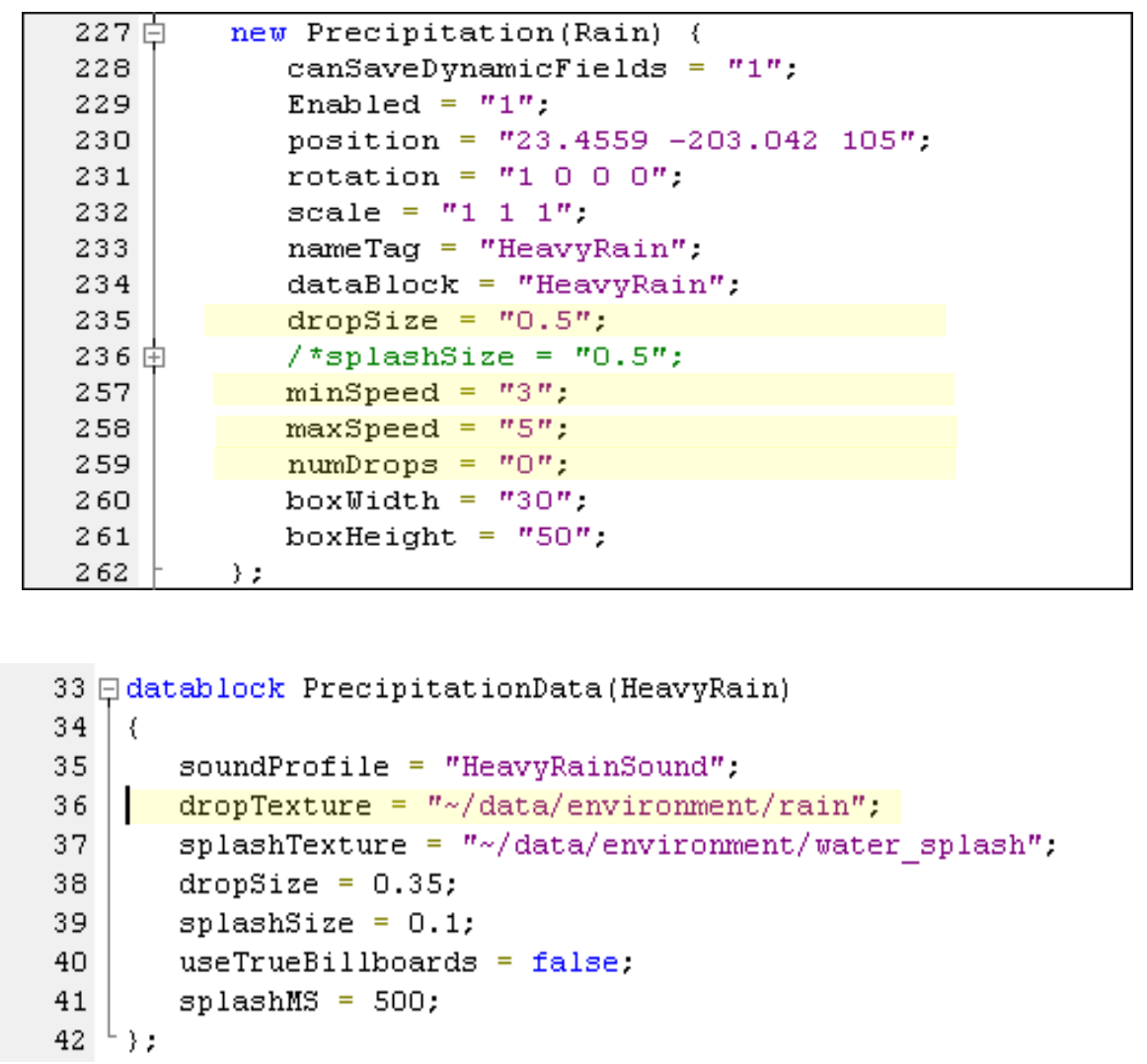

Code Extract 3.1: Datablock of precipitation

The simulation of precipitation is mainly determined by 3 factors: density, type and velocity.

1). Density determines the amount of precipitation, e.g., whether it is a drizzle or a downpour. It is specified by 2 parameters in the "Precipitation" datablock: "numDrops", the number of precipitation drops in the scene; and "percentage", the percentage of drops presented in view. The relationship between the real precipitation amount and the density in the virtual game can be expressed as:

$P A=k *$ numDrop $*$ percentage equation3.6

In this equation, $P A$ stands for the precipitation amount in the real world, where $k$ is 
determined by the hardware, and is fixed when the scenario starts.

2). Type indicates whether the precipitation is rain, snow, or hail, and is represented by "droptexture" in the "PrecipitationData" datablock. It contains a relative path to a PNG file, which is a $4 * 4$ grid containing one precipitation drop image in each of the 16 grid blocks. When the engine gets ready to produce a new drop, it will randomly select one of the 16 sub-textures and use it as the precipitation billboard (Figure 3.23).

Figure 3.23: Precipitation grid blocks

3). Velocity: Real raindrops do not all fall onto the earth at the same rate. Therefore, the "Precipitation" datablock has the ability to randomly vary the velocity of individual drops. Setting the "minVelocity" and "maxVelocity" in "Precipitation" datablock causes the raindrops to fall at a random speed value within the two threshold values. This functionality can improve the fidelity, but it does not affect the amount of precipitation.

Figure 3.24 gives some examples of different precipitation conditions. The condition can change with time as new real world data is available. 


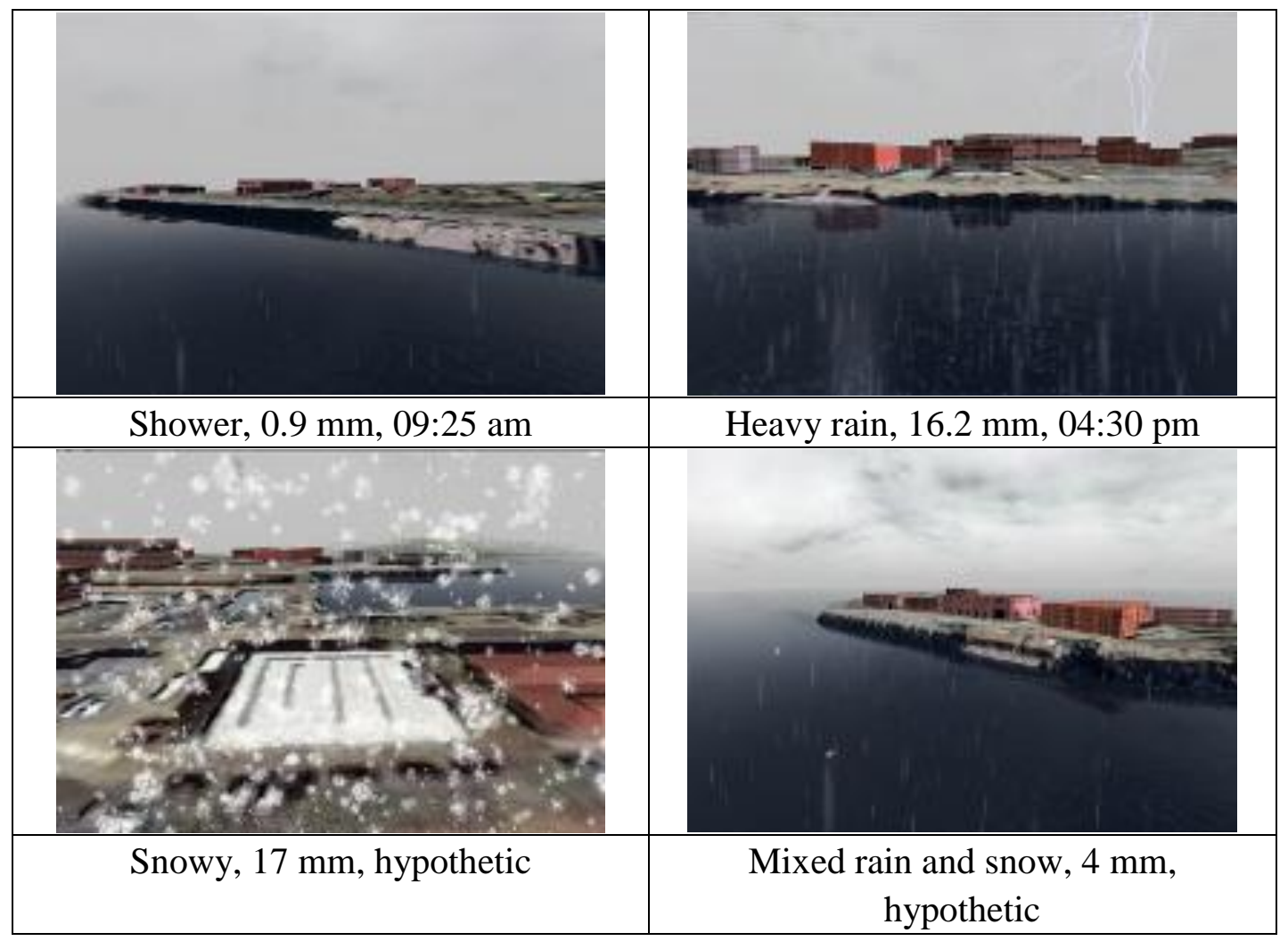

Figure 3.24: Different types and amounts of precipitation

Daylight refers to the combination of all direct and indirect sunlight during daytime. In the real world, daylight information can be acquired, including sunrise and sunset time, and illumination intensity. In the virtual world, daylight is determined by parameters of "Sun" datablock (Code Extract 3.2).

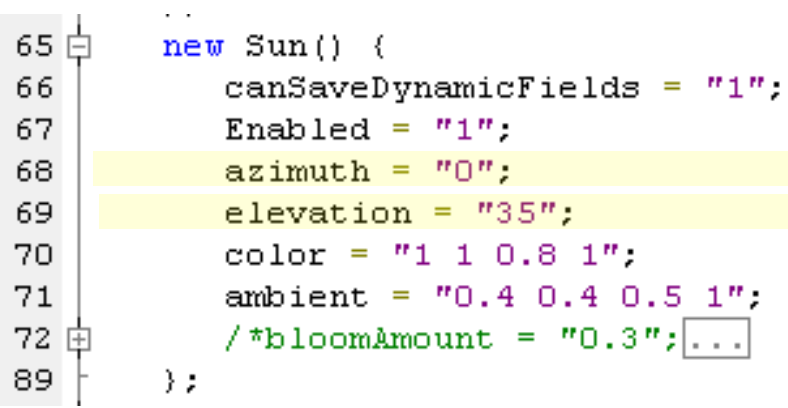

Code Extract 3.2: Datablock of sunlight

Sun datablock uses elevation and azimuth to represent the position of the sun in the sky. In a spherical coordinate system, elevation refers to the vertical angle measured from the astronomical horizon towards the zenith, which ranges from $0^{\circ}$ to $90^{\circ}$. Azimuth is defined as the angle between the projected vector and the due north in a 
clockwise direction, which ranges from $0^{\circ}$ to $360^{\circ}$. While the position of the sun is regularly changing from the time of sunrise to sunset, defining the model of solar azimuth and elevation is the key task of simulation. The approximation formulae are: Elevation : $\sin \theta_{s}=\cos \hbar \cos \delta \cos \Phi+\sin \delta \sin \Phi$ equation3.7

Azimuth $: \sin \phi s=-\sin \hbar \cos \delta / \cos \theta$ equation 3.8

where $\theta_{s}$ is the solar elevation angle, $\phi_{s}$ is the solar azimuth angle, $\hbar$ is the hour angle of the present time, which is determined by local time, $\delta$ is the current sun declination, which is related to the date of year, and $\Phi$ is the local latitude.

Implementing daylight simulation has the advantage of modelling shadows and brightness. Generally objects are darker on the opposite side to that facing the sun, and the shadow formed changes with the position of the sun. Shadows and brightness can be properly and automatically calculated based on the azimuth and elevation parameters. In the virtual environment, the position of the sun affects two other parameters, light colour and ambient light level, which accounts for the total lighting of the objects. The illumination functions can be implemented using a time controller to dynamically change the elevation and azimuth in the datablock. Finally the solar position and daylight can be simulated in the SIEVE-VTS (Figure 3.25). 


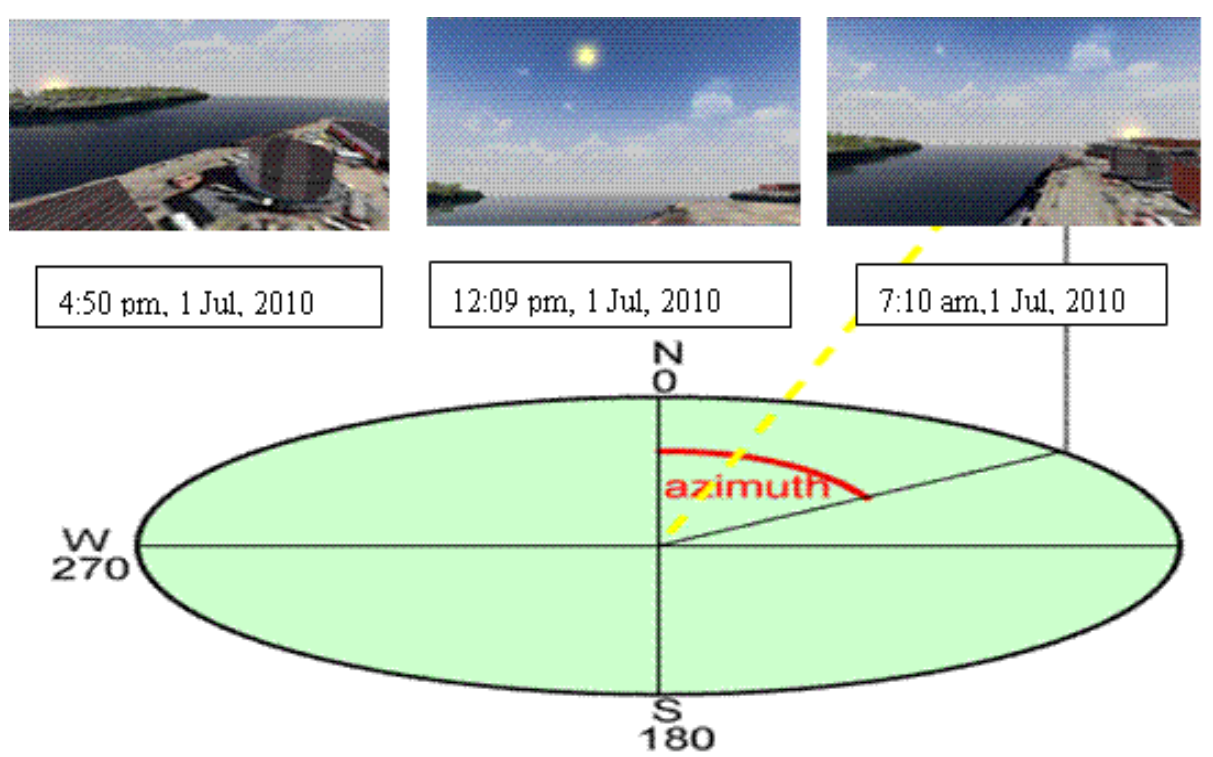

Figure 3.25: Simulation of daylight in SIEVE-VTS

Basic weather condition, wind status, and visibility are three important weather elements for simulating. They are all closely related to the definition of atmosphere in the virtual environment. In RTDSimulator, these weather elements are coded in the "SkyObject” datablock (Code Extract 3.3).

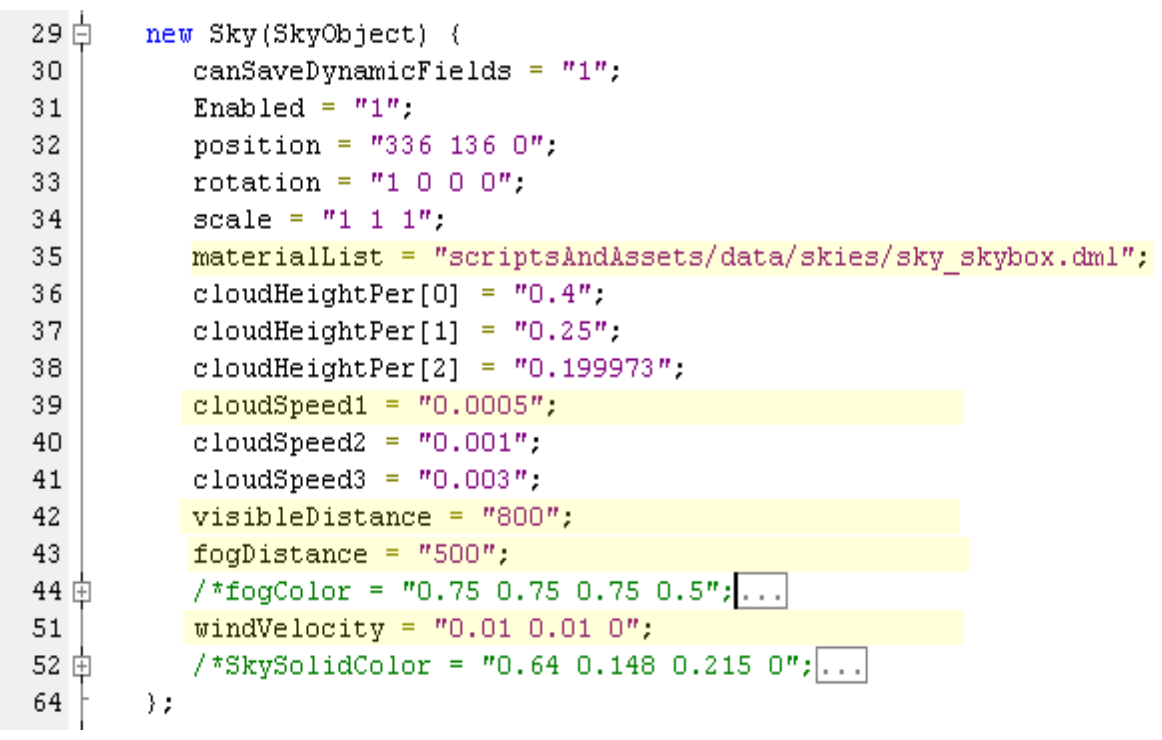

\section{Code Extract 3.3: Datablock of sky}

RTDSimulator applies a force vector to simulate the horizontal movement of the air, which in the real world is measured as wind speed and direction. "windVelocity" is represented as a $3 \mathrm{D}$ vector and describes wind features including both direction and 
speed. Although the wind cannot be seen in either the real world or in the virtual world, the behaviour of floating objects can be used to exhibit the wind. The integrated parameter, "windEffect", can be switched on and off, therefore particles such as explosion debris, precipitation and dust are indirectly affected by "windVelocity". "Cloudspeed" is another parameter used to represent the wind.

Visibility is mainly controlled by "visibleDistance" which defines the maximum view distance in virtual world units. Visibility can also be affected by extreme weather conditions such as fog, and the "fogDistance" parameter in the "SkyObject" datablock controls the visibility in foggy conditions (Figure 3.26).

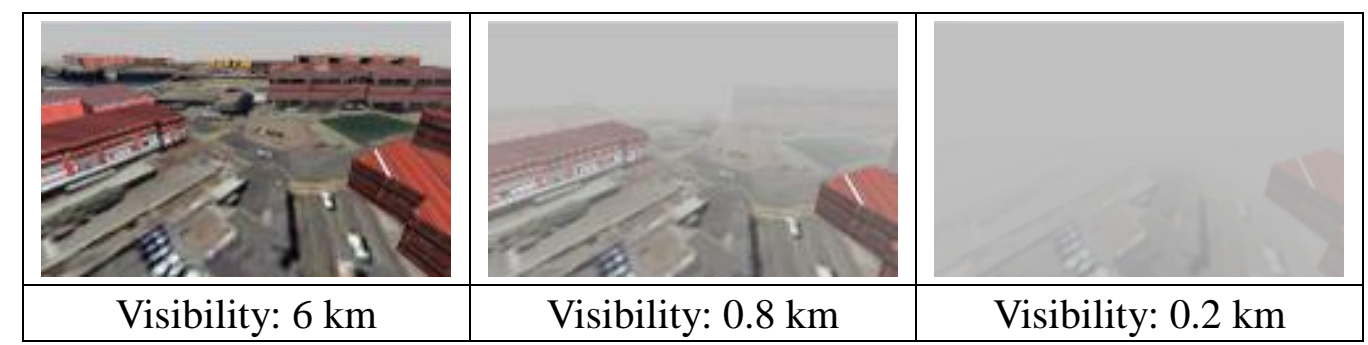

Figure 3.26: Visibility in SIEVE-VTS

As the auxiliary information source, Yahoo! Weather provides a basic description of the weather condition. The parameter of "materialist" in the SkyObject defines the material and texture related to the weather conditions. It points to a DML file (Code Extract 3.4) which lists the names of images that make up the skybox and cloud layers. It contains textures for the sky (line 1-7) and clouds (line 8-10).

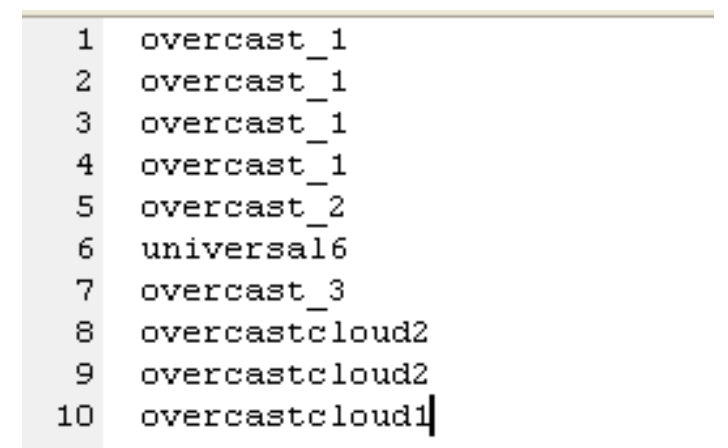

Code Extract 3.4: DML file 
Four basic weather templates are designed to correspond to the 48 different Yahoo! weather codes (Table 3.4). Weather codes are indexed to represent different detailed weather conditions (Appendix 1). When simulating weather in this way, precipitation, daylight, wind, and visibility need to be adjusted to simulate the conditions as accurately as possible, based on real-time data. For example, if the weather condition is "scattered showers" (Weather Code 40), the basic weather template "rainy" is selected, and parameters such as precipitation, wind status, visibility are tuned using real-time weather data.

\begin{tabular}{|c|c|c|}
\hline Basic weather & Weather Code & Snapshot \\
\hline Sunny & $31-34,36$ & \\
\hline Rainy & $0-20,35,37-47$ & \\
\hline Cloudy & $25-30$ & \\
\hline Else & & \\
\hline & & \\
\hline
\end{tabular}

Table 3.4: Weather templates

Of all the parameters in the real-time weather information, some can be simulated in the virtual environment, while other elements such as atmosphere pressure and temperature cannot be directly visualised in SIEVE-VTS. They can either be stored in the database for analysis, or displayed in the GUI as complementary data for emergency management (Figure 3.27). 


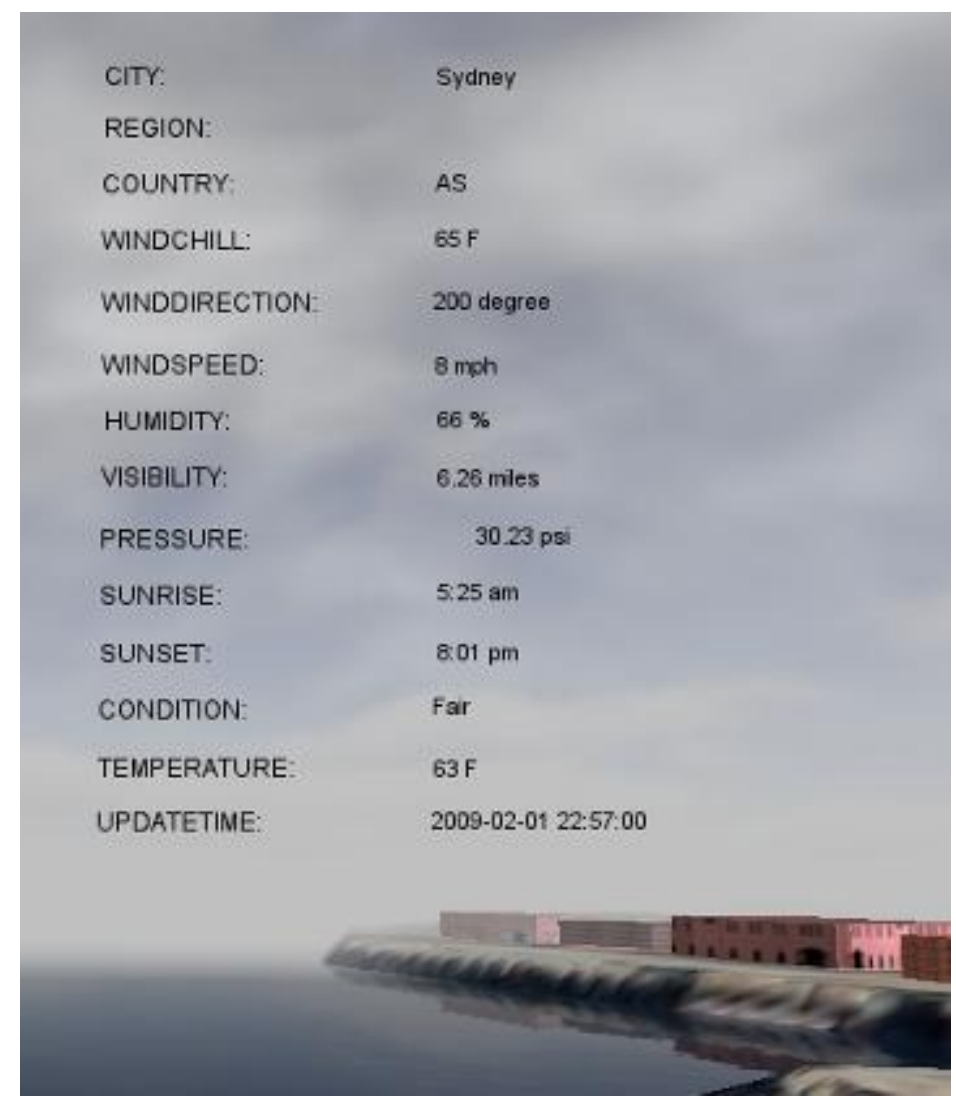

Figure 3.27: Non-simulated data in GUI

\subsubsection{Oceanographic information simulation}

An oceanographic information system contains information about sea level, tides, waves, sea surface temperature, salinity, as well as maritime geophysical and geology data. Such data can be utilised in products and services which are delivered to resource managers and policy makers around the world to enhance maritime security, and manage natural and man-made disasters. Visualising the comprehensive datasets in the virtual environment may be desirable but is impractical. As previously discussed, real-time tide and swell information was collected because it is representative, readily available and frequently updated. In SIEVE-VTS, by manipulating and modifying the datablock of "WaterBlock" (Code Extract 3.5), the simulation of tide and swell using real-time data can be implemented. 


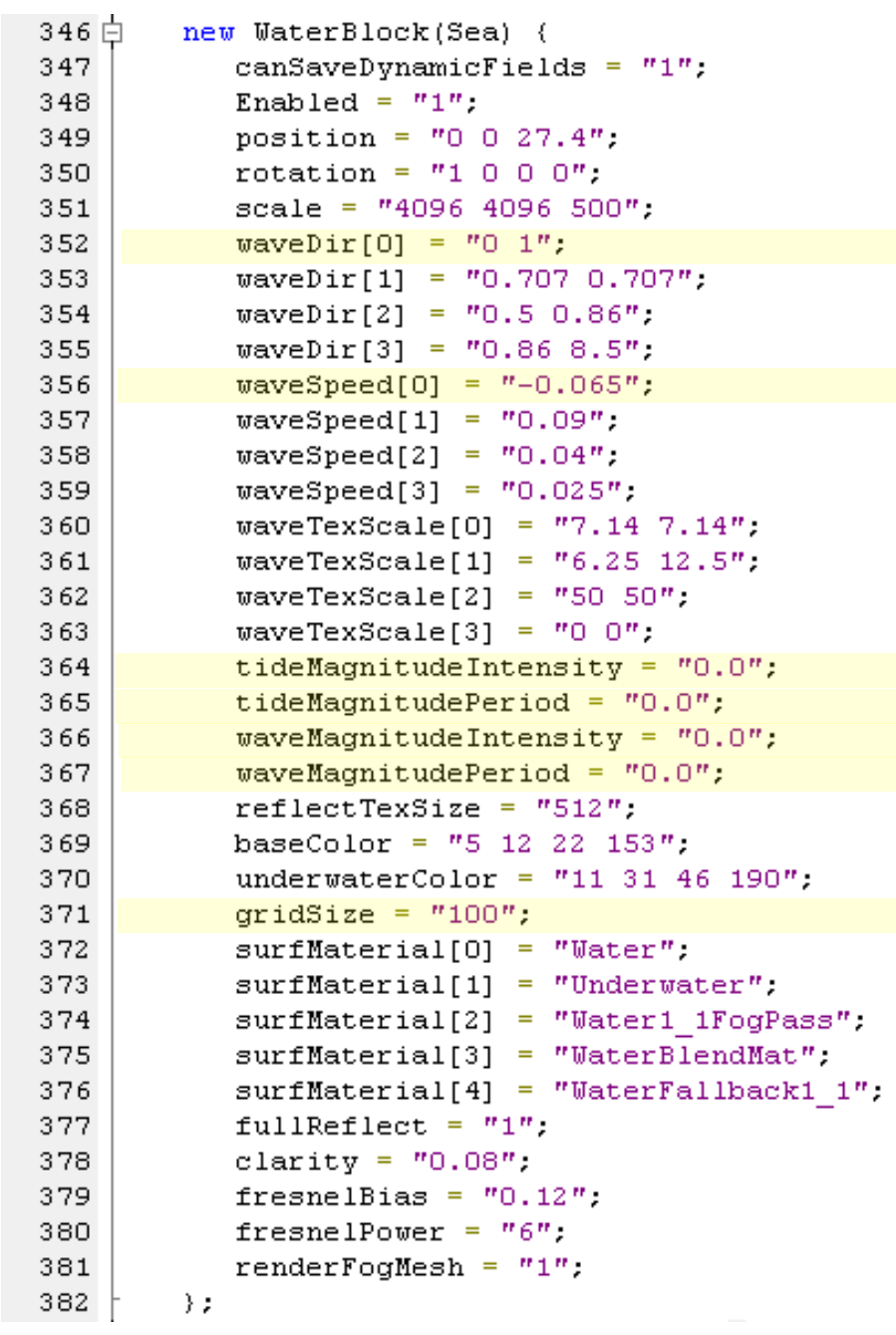

Code Extract 3.5: Datablock of waterblock

TGEA integrates a shader-oriented waterblock system that can be customised to simulate water with high-level visual effects. The main parameters in the "waterblock" can be divided into the following categories:

1. Waterblock transform and shape: The water is defined as a square shape and is set up as a brute-force grid mesh. Similar to other objects defined in TGEA, the position, rotation, and scale are used to control the dimensions and position of the water body.

2. Reflection: Waterblock is capable of providing full dynamic reflections. All the visible objects in the virtual environment are reflected in the scene. The reflections are updated at roughly $60 \mathrm{~Hz}$. In order to reduce the cost to system resource, full 
reflections can be degraded to static cube map reflections controlled by the "fullReflect" parameter.

3. Water types: The type of water can be altered by defining various types of water surface material. Using different materials can represent different types of liquids, such as sea or lava. In this research, sea water material was selected, which is a common option for marine environment simulation.

4. Phase and altitude of tide and swell: Tide and swell are abstracted as the horizontal and vertical movement of sea water. Since the original TGEA has no parameters for controlling the phase and altitude, four parameters were added into "WaterBlock", controlling the spatial and temporal change of sea water in vertical direction. "tideMagnitude" and "tideFrequency" define the intensity and changing rate of tides in units of metres and hertz, respectively. Accordingly, "swellMagnitude" and "swellFrequency" define the same attributes of swell.

5. Horizontal wave data: This is used to simulate the horizontal movement of water. The parameters for the waves are: "waveDir", a 2D normalised vector controlling the direction that the wave travels along a plane, and "waveSpeed", a floating point value defining the speed that the wave travels in the specified direction.

The real-time tide information provides height and time of high tide and low tide for each day. To obtain tidal data at other times, appropriate models need to be implemented. However, it is usually difficult to accurately model tidal change because it is influenced by multiple factors, including the earth's rotation, the relative position of sun and moon to the earth, the moon's altitude above the earth, and the bathymetry. Models are commonly used to abstract and approximate the tide, and these models range from simple to complex ones (Lyard, Lefevre, Letellier, \& Francis, 2006; Egbert \& Erofeeva, 2002; Boon, 2004). A simple model called "the rule of twelfths" can suffice if a more accurate model is not available (DIY Wood Boat, 2010). It presumes that the increase in depth during the six hours between low and high tide is: 
$1 / 12$ for the first hour, $2 / 12$ for the second, $3 / 12$ for the third, $3 / 12$ for the fourth, $2 / 12$ for the fifth, and 1/12 for the sixth. Therefore, it can approximately predict the water level on an hourly basis. Conversely, the HAMELS method is a complex model that sums up nine tidal constituents (Virginia Institute of Marine Science, 2010):

$H(t)=M_{2}+S_{2}+N_{2}+K_{1}+O_{1}+M_{4}+M_{6}+S_{4}+M S_{4}$ equation 3.9

In equation 3.9, each symbol on the right side of the equal sign is identified as the tidal constituent, and can be represented as a cosine wave with a suitable frequency. The general type of tidal constituent is:

$h(t)=R * \cos \left(q t+v_{0}+u-k\right)$ equation 3.10

in which $h(t)$ is the height of tidal constituent, $R$ is the tide amplitude, $q$ is the angular velocity, $t$ is the local time, $v_{0}+u$ is the phase angle, $k$ is the epoch of the tidal constituent. These parameters can either be looked up in astronomy tables or calculated under certain circumstances. By adding the nine constituents together, tide height can be calculated.

In SIEVE-VTS, simulating the tidal change requires a trade-off between efficiency and accuracy. An appropriate model is designed to meet both the requirement of computational efficiency when rendering a scene, and the accuracy in representing real-time data. The main steps for the tide simulation are (Algorithm 3.1):

- Before the simulation starts, the algorithm verifies if the value of tide period $p$, and tide intensity $i$ are valid for calculation (line $1.1-1.2$ ).

- Then the function computes the water level offset using offset $=$ intensity $* \sin (x)$, which treats tidal change as a sine function. An accumulator is also assigned for the current time period (line $1.3-1.5$ ).

- Finally, a function is called to set the new water level in the virtual environment 
(line 1.6).

- The calculation is reset when a full tide cycle has been completed (line $1.7-1.8$ ).

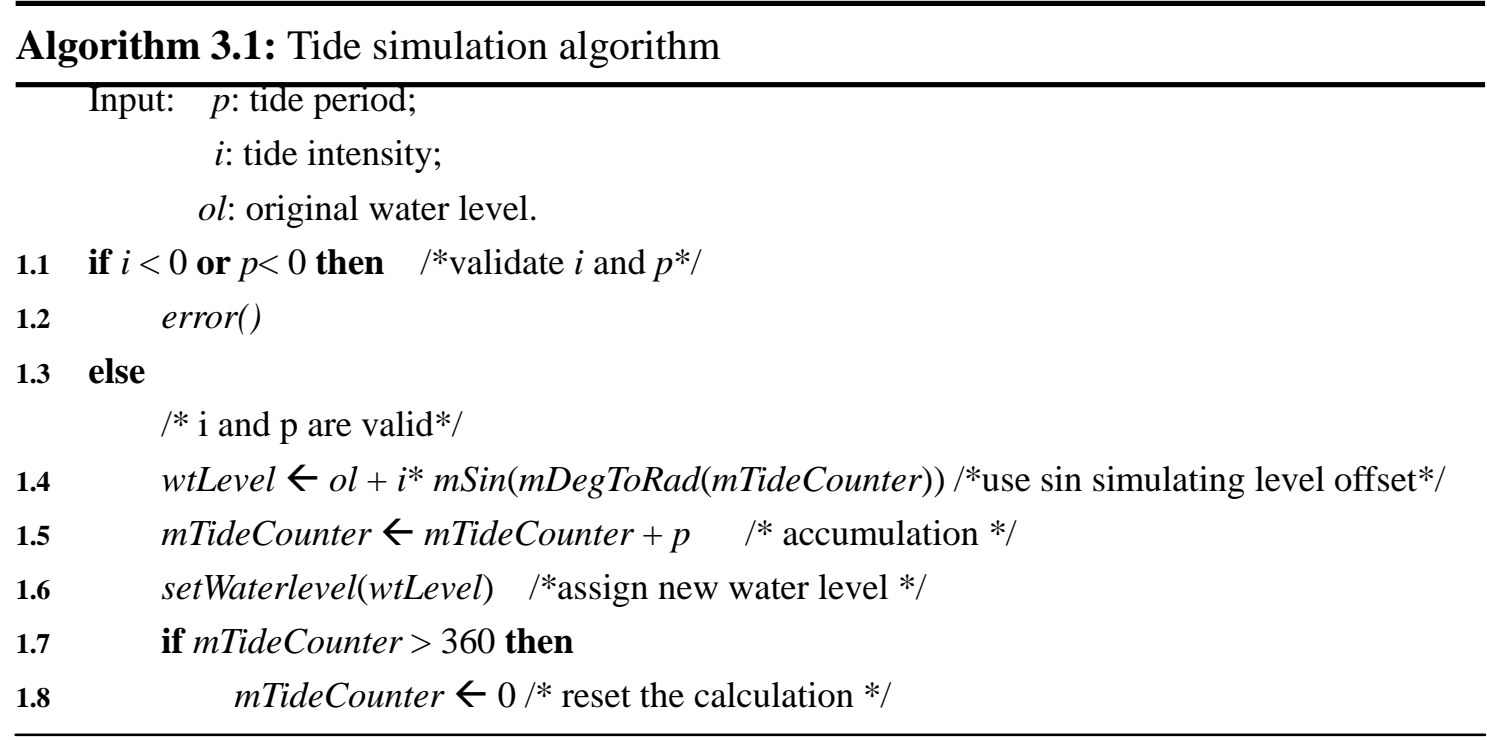

In this algorithm, $p$ and $i$ are input values which are calculated using the real-time tide information (Section 3.5.1). When a new dataset is updated, the values of $p$ and $i$ are renewed and assigned to new calculation. This algorithm is implemented in a rendering function which renders the scene in each frame. Finally, the water level will rise and drop regularly with time in a sinusoidal motion, which is approximately representative of the real tide (Figure 3.28).

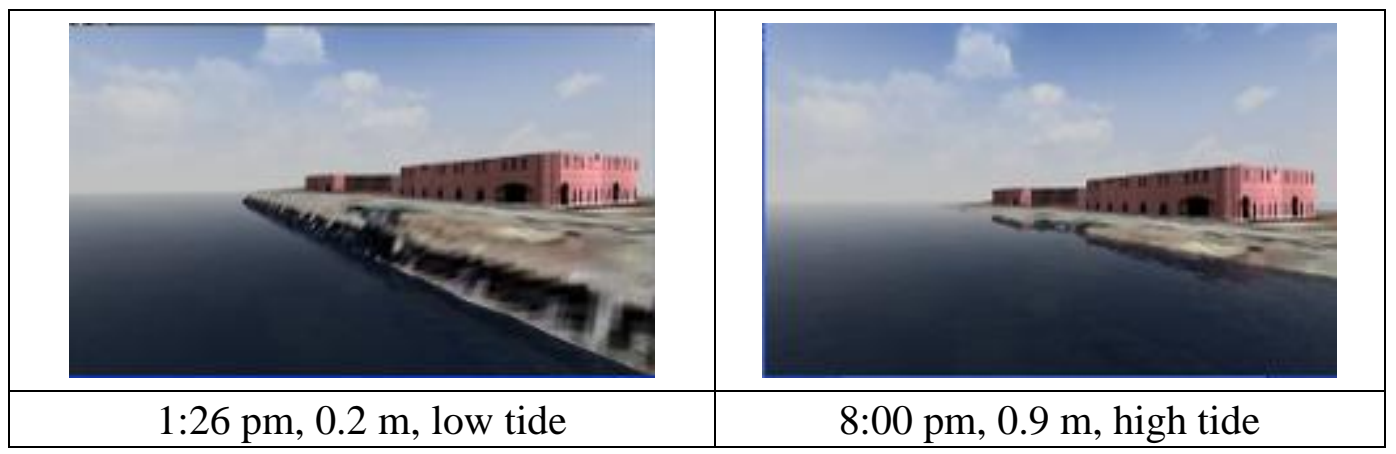

Figure 3.28: Tide simulation in SIEVE-VTS

Swell height refers to the average of the highest one-third of swell waves. For example, about $10 \%$ of the waves are higher than the indicated level, $1 \%$ are more than $50 \%$ higher, and $0.1 \%$ are approximately twice as high. The same ratio applies 
for waves lower than the indicated level. Swell period implies the distance between swell waves. It refers to the amount of time it takes for two successive wave crests to pass a fixed point. The exact height and period of the swell will also depend on the contour of the seabed, wind speed and direction (Willy Online Pty Ltd, 2010).

Swell is more complex to simulate than tide. It not only contains height and period in its vertical movement, but also includes speed and direction in its horizontal movement. Observation data can be accessed every 3 hours from $B O M$, and data at other times can be interpolated.

Algorithm 3.2 describes the procedure for simulating the swell:

- The input values: swell period and swell intensity, are confirmed valid for calculation (line $2.1-2.2$ ).

- For horizontal movement, users can obtain wave speed and direction from real-time swell data and convert it to values in the virtual environment (line $2.3-2.6$ ).

- For vertical movement, the function computes the water level offset using offset $=$ intensity $* \sin (x)+$ increment, which defines swell as a sin function with minor random increments (line 2.7).

- Similar to tide simulation, new water levels are set automatically and calculation is reset when one cycle is completed (line $2.9-2.11$ ). 
Algorithm 3.2: Swell simulation algorithm

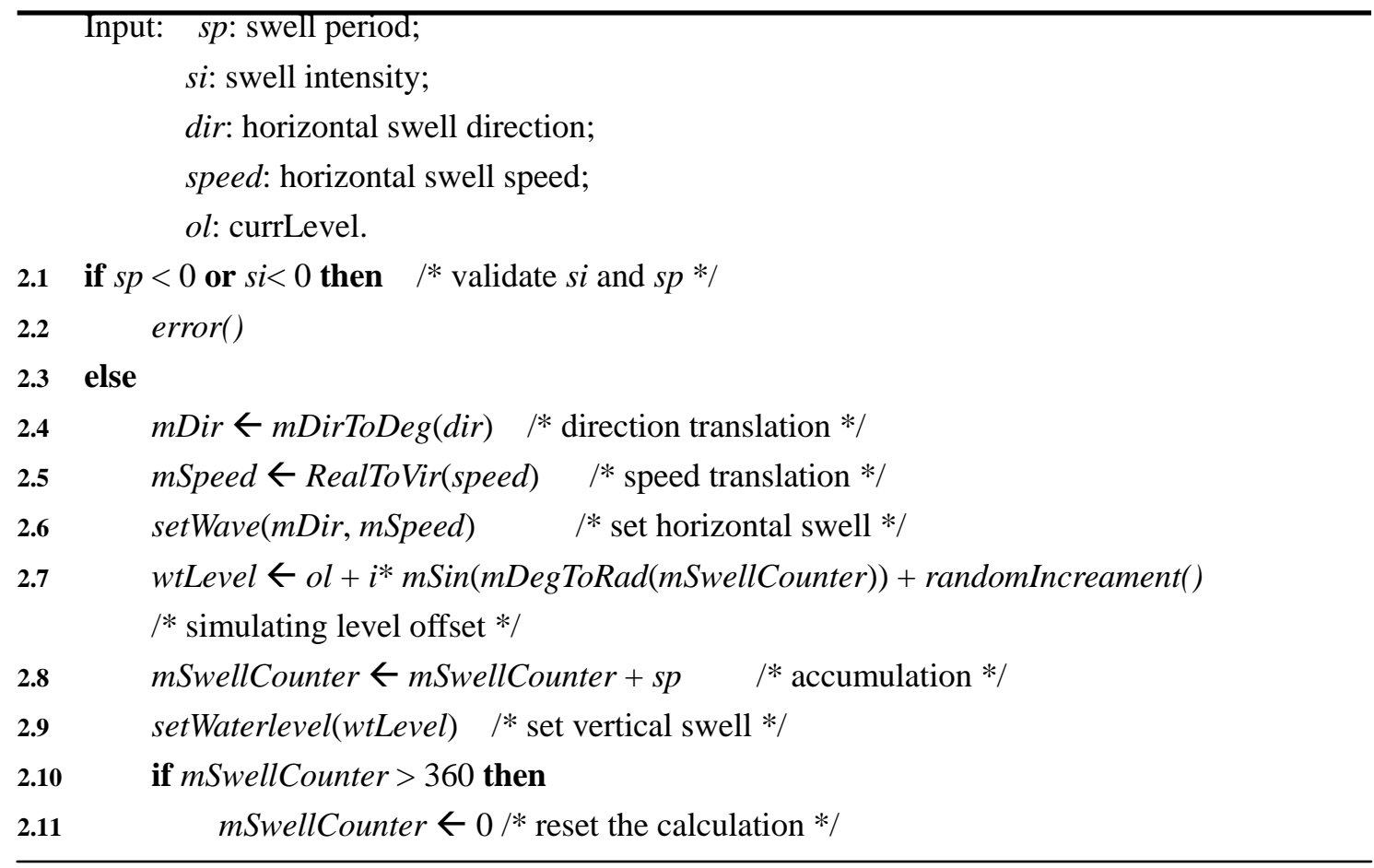

The water heights given by the tide and swell algorithms are combined to define the overall water shape and movement. This is of great significance in an emergency situation, for example:

- During high tide, a vessel can pass through a certain area, however in low tide, it may not be able to.

- Certain coastal features or infrastructure, such as wharves or storm water pipes, may or may not be visible or accessible in the high tide and swell condition.

- The visibility of distant vessels will change with the tide.

- Swell levels can affect the ability of people to transfer between vessels.

\subsubsection{Shipping simulation}

In order to simulate shipping in SIEVE-VTS, particularly marine traffic in inshore and harbour areas, a vessel class was specifically designed and integrated into TGEA. 
Object-oriented programming was used to implement the main components and to represent relationships, such as inheritance and composition as classes. A hierarchical view of these classes is given in Figure 3.29:

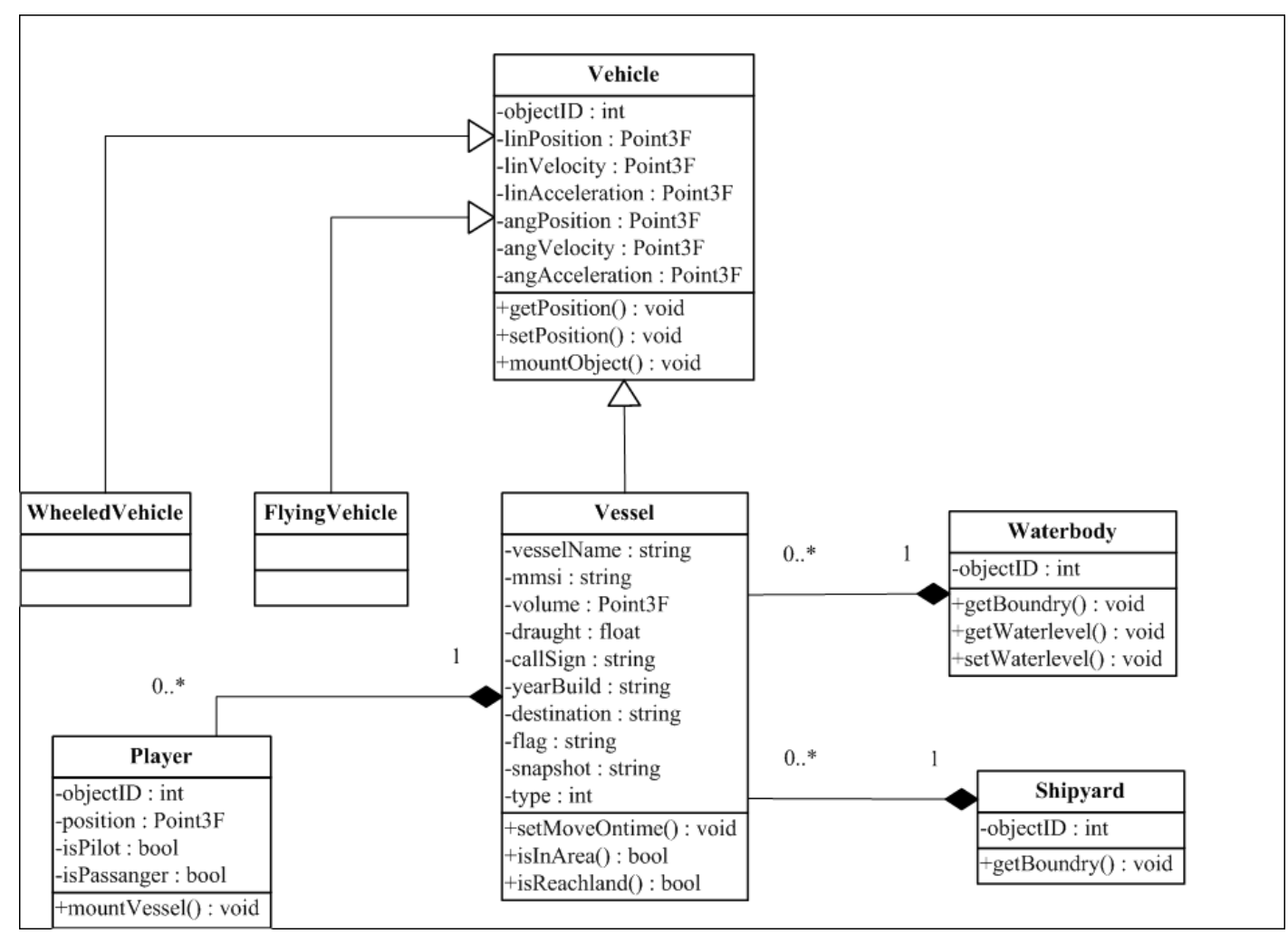

Figure 3.29: Class diagram of vessel in SIEVE-VTS

The vessel class is derived from the existing vehicle class, with the main elements as follows:

ObjectID: It is inherited from the parent class "Vehicle", indicating the unique identification number for any object in the virtual world.

Spatial information: linPosition and angPosition represent the linear and angular position of the vessel. A point $3 \mathrm{~F}$ data type (float $\mathrm{X}$, float $\mathrm{Y}$, float $\mathrm{Z}$ ) is used to represent the current position of the vessel. The $\mathrm{x}$, $\mathrm{y}$ values are converted from the longitude and latitude in AIS data into virtual location coordinates, and the $\mathrm{z}$ value determines the altitude of the vessel near the sea surface and is conditioned by the draught of the vessel. 
Movement: linVelocity, linAcceleration, angVelocity and angAccelaration are used to define the motion state of the vessel, which is translated from the speed and course in the AIS data. Such data only represents the instantaneous speed of the vessel at a certain location and time, therefore an acceleration value needs to be calculated using: Acc $=(v N e x t-v L a s t) / t N e x t-t$ Last equation3.11 or $A c c=\left(v\right.$ Last $_{1}-v$ Last $\left._{2}\right) / t$ Last $_{1}-$ tLast $_{2}$ equation 3.12

In equation 3.11, tNext and $v$ Next represent time and velocity data in the next record, tLast and vLast represent time and velocity data received in the last record. The acceleration can be used for interpolating velocity at any other time which is not recorded in the database. This is ideal for emergency training using historical data. In equation 3.12, tLast $_{1}$ and $v$ Last $_{1}$ represent time and velocity data in the last record, $t$ Last $_{2}$ and $v$ Last $_{2}$ represent time and velocity in the one before the last record. This is used for extrapolating the velocity of the unknown point in real-time situations such as emergency monitoring.

The direction of the vessel is a floating value ranging from 0 to 360 degrees. This data is similar to velocity in that it represents an instantaneous value. Therefore, it is necessary to calculate the direction change rate, and determine the direction value at any time between two historical records (equation 3.13) or as a prediction for the future record (equation 3.14).

$A c c \_d i r=(d N e x t-d L a s t) / t N e x t-t$ Last equation 3.13

$A c c_{-} d i r=\left(d\right.$ Last $_{1}-d$ Last $\left._{2}\right) / t$ Last $_{1}-t$ Last $_{2}$ equation 3.14

Other vessel attributes include information about the vessel itself, such as draught, type, volume, ship name, MMSI, and year built.

Draught is used to determine the depth of the vessel underwater. In the real world, 
draught is usually defined as the vertical distance between the water level and the bottom of the ship. In the virtual environment, draught can be represented by adjusting the relative height of a vessel to the water surface. Draught is useful for navigation as it determines the minimum depth of water in which a ship can safely travel.

Type is a string value defining the type of vessel. Taking examples from the real world, vessels are categorised into 6 groups: passenger vessel, cargo vessel, tanker, high speed craft, pilot vessel, and unspecified type. Each type contains a relative path to a vessel model in the object library. The models are pre-designed in $.3 d s$ format using 3D Studio Max and converted into .dts format which is supported by SIEVE-VTS. Since designing a unique model for each boat is time consuming, each type of vessel is assigned to one model to simplify the process (Table 3.5), and individual ships of the same type look different only according to their dimensions. When the object library is populated in the future, it may be appropriate to expand the range of modelled types. 


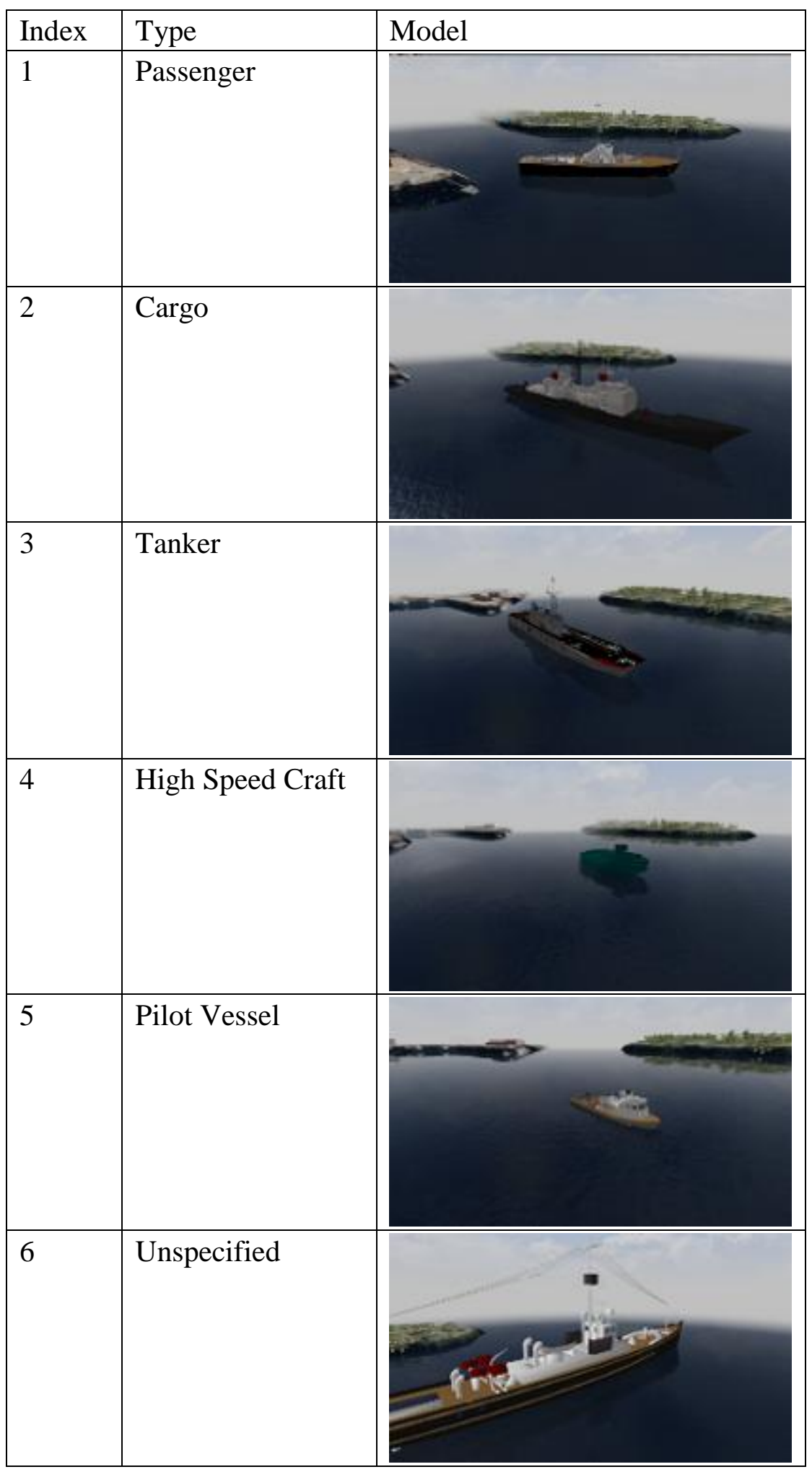

Table 3.5: Ship types and models

Volume is vector data comprising length, width and height. It can be translated into virtual units, and demonstrated via appropriate scaling of the ship model. Ship name, 
MMSI, built year cannot be simulated directly in SIEVE-VTS; this complementary information is kept available for optional display in the GUI.

Methods for vessel movement: new methods were especially designed to define the movement of vessels. setPosition(pos) is invoked when initialising the state of vessels. getPosition() returns the current position of the object. setMoveOnTime(curr_pos, next_pos, curr_vel, next_vel, curr_c, next_c, curr_time, next_time) sets the vehicle moving from current position to the next position during the current time and next time point. It interpolates the vessel's motion trail between the current position and next position in the location list using the known course and speed, or extrapolates the vessel's position and velocity using the acquired real-time AIS data. Algorithm 3.3 describes the interpolation of the movement of a single vessel between two points. Firstly, the angular velocity and linear acceleration of the vessel, and the total frame are calculated based on the observed spatial data (line $3.1-3.4$ ). In each frame, the position, speed and course of the vessel are interpolated (line $3.5-3.9$ ). Finally the new position and velocity of the vessel are reset (line 3.10-3.11). 
Algorithm 3.3: Single vessel movement algorithm

$\begin{array}{ll}\text { Input: } & p_{1}: \text { location at last available point; } \\ & p_{2}: \text { location at next available point; } \\ & v_{1}: \text { speed at last available point; } \\ & v_{2}: \text { speed at next available point; } \\ & c_{1}: \text { course at last available point; } \\ & c_{2}: \text { course at next available point; } \\ & t_{1}: \text { time at last available point; } \\ & t_{2}: \text { time at next available point; } \\ \text { ticktime: time per frame. }\end{array}$

3.1 if $t_{1}<t_{2}$ then $/ *$ validate time*/

3.2 ang_vel $\leftarrow\left(c_{2}-c_{1}\right) /\left(t_{2}-t_{1}\right) \quad / *$ calculate the angular velocity $* /$

3.3 lin_acc $\leftarrow\left(v_{2}-v_{1}\right) /\left(t_{2}-t_{l}\right) \quad / *$ calculate the linear acceleration $* /$

3.4 Total_frame $\leftarrow\left(t_{2}-t_{1}\right) /$ ticktime $/ *$ calculate the total frames $* /$ $k \leftarrow 0$

$3.5 \quad$ While $k<=$ Total_frame do

3.6 $\quad x_{k} \leftarrow p_{1} . x+v_{1} * \sin \left(c_{1}\right) *$ ticktime $* k+0.5 *\left(v_{2}^{*} \sin \left(c_{2}\right)-v_{1} * \sin \left(c_{1}\right)\right) /\left(t_{2}-t_{1}\right) *$ pow(ticktime $* k, 2)$

$3.8 \quad c_{k} \leftarrow c_{1}+$ ang_vel $^{*}$ ticktime $* k \quad l *$ calculate the course of vessel at next interpolation time $* /$

3.9 $\quad v_{k} \leftarrow v_{l}+$ lin_acc $*$ ticktime $* k \quad / *$ calculate the speed of vessel at next interpolation time $* /$

$3.10 \operatorname{SetPosition}\left(x_{k}, y_{k}\right)$

3.11 $\quad \operatorname{SetVel}\left(c_{k}, v_{k}\right) \quad$ set new conditions for the vessel */

$3.12 k++$

Methods for judging vessels' area: after determining the attributes and movement of the vessel, the next challenge is judging where the vessels are located. This is important for simulating multiple vessels' behaviours.

isInArea(): a function which returns a Boolean value is implemented to determine whether a vessel is within the boundary of the area of interest. If the vessel is found to be outside the area of interest, it will be set to invisible.

isReachLand(): a function which returns a Boolean value is used to determine whether a vessel moves out of water, such as colliding with land or other objects. Once the function returns a true value, either the vessel is set to static state immediately, or it 
triggers other incidents such as explosion or sinking.

Mountobject(): The vessel is originally set in automatic navigation state using the AIS data. The Mountobject() function is called for accommodating players or other objects in the vessels. In this way, a player can control the vessel using mouse and keyboard.

Algorithm 3.4 is designed for global simulation of AIS supported shipping. It first reads the vessel information from the database and creates a vessel model in the virtual environment (line $4.1-4.2$ ). It then checks whether the vessel is in a valid area or at the boundary of water (line $4.3-4.6$ ). A moving vessel within a valid area is assigned an initial value which is retrieved from the database. The movement route is calculated, and a SPLINE curve method is used to smooth the route using algorithm 3.3 (line $4.7-4.9$ ). If a new record is received, it will be assigned the next location, while the next location data in the previous step will be assigned the current location (line $4.10-4.14)$. The process will be repeated until each vessel in the database is given a suitable model, a position and a movement path.

\section{Algorithm 3.4: AIS algorithm}

4.1 While nextship $=$ TRUE do

4.2 $\quad$ Ship $[i] \leftarrow$ new Ship (shiptype, shipname, volume, location)

4.3 If ship[i]. isInArea( $)=$ FALSE

4.4 Warn(ship[i]. shipname + "is not in Sydney Harbour")

4.5 Else if $\operatorname{ship}[i]$. isReachLand $=$ TRUE

4.6 Warn ("ship [i]. shipname + "anchored!")

4.7 Else

4.8 While $($ record $\neq$ NULL $)$ do

4.9 Ship[i]. interpolate(pos_cur,pos_next,cou_cur, cou_next, vel_cur, vel_next,

t_cur,t_next) /*set new conditions for the vessel*/

4.10 pos_cur $=$ pos_next

$4.11 \quad c o u \_c u r=c o u \_n e x t$

$4.12 \quad v e l \_c u r=v e l \_n e x t$

$4.13 \quad t \_c u r=t \_n e x t$

4.14 Record $\leftarrow$ nextRecord

4.15 Warn("Ship reached destination!")

4.16 i++ 
In an emergency response situation, the real-time AIS data can be displayed in a 3D virtual environment, including the ship attributes and routing information. Ship activities such as arriving and leaving the port, anchoring or moving from one location to another, may provide emergency managers with useful information when monitoring suspicious ships, predicting potential dangers, avoiding the risk of collision and assisting rescuing impaired vessels. In an emergency training circumstance, trainees can observe historical shipping information in the virtual environment to familiarise themselves with situations in the harbour area. Furthermore, they can also use the avatars to manipulate the vessels, such as moving, convoying, communicating or colliding with other vessels. Ultimately, this experience will enhance the trainees' decision making ability when facing a similar situation in the real world.

\subsubsection{Incident location and status simulation}

A game engine is an ideal platform for incident scenario simulation, such as fire and explosion incidents. Such simulation is based on particle engine as detailed in Section 3.4. SIEVE-VTS makes further steps by utilizing real-time data collected from mobile devices, and simulating it in virtual environments. Any such incident can be modelled as a "ParticleEmitterNode" in TGEA (Code Extract 3.6).

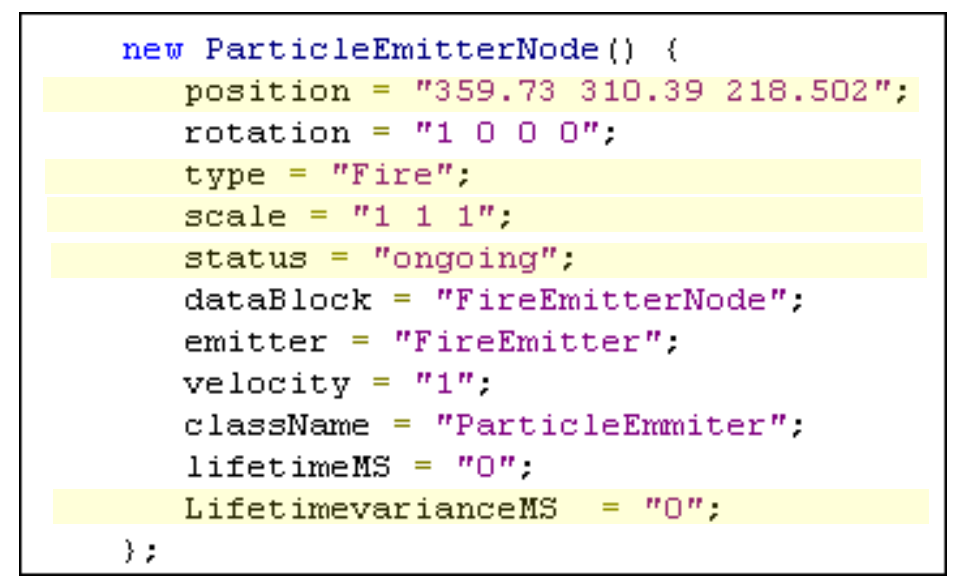

Code Extract 3.6: Datablock of incidents 
In the incident datablock, position is defined as the location of the incident in virtual units, which is translated from the real world data provided by the iPhone. Detailed translation procedures were introduced in Section 3.6.1. Type is represented by three specially designed incident types: explosion, fire and gas leak (Figure 3.30), and it is determined by the datablock parameter which connects to different incident models.

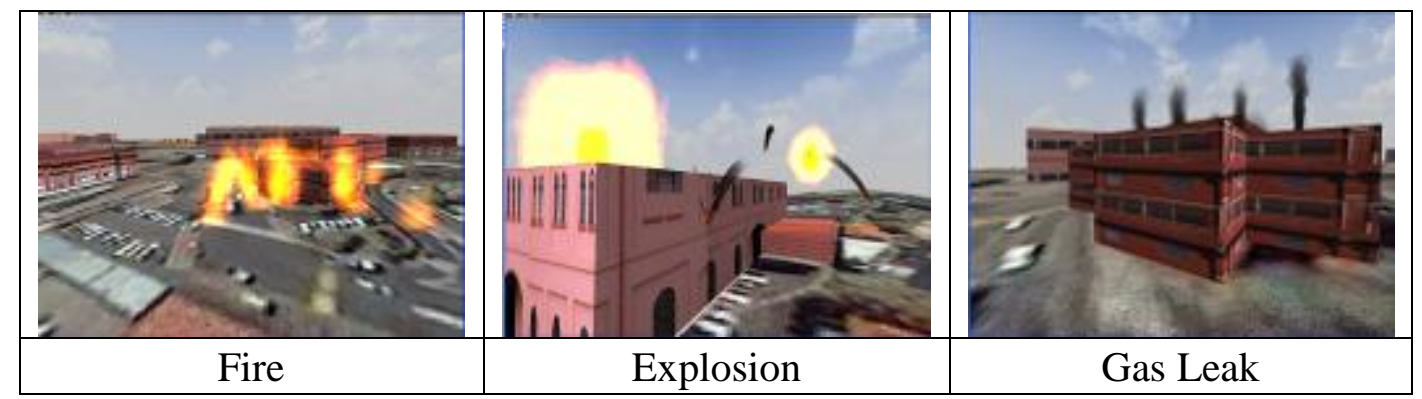

Figure 3.30: Type of incidents

Scale in the incident datablock is determined by the area of the incident in the real world. It is captured and recorded by the iReport application at the stage of real-time data collection, and is categorised as large (more than $500 \mathrm{~m}^{2}$ ), medium (100-500 $\mathrm{m}^{2}$ ), and small (0-100 $\mathrm{m}^{2}$.) scale, which also indicates the level of danger (Figure 3.31).

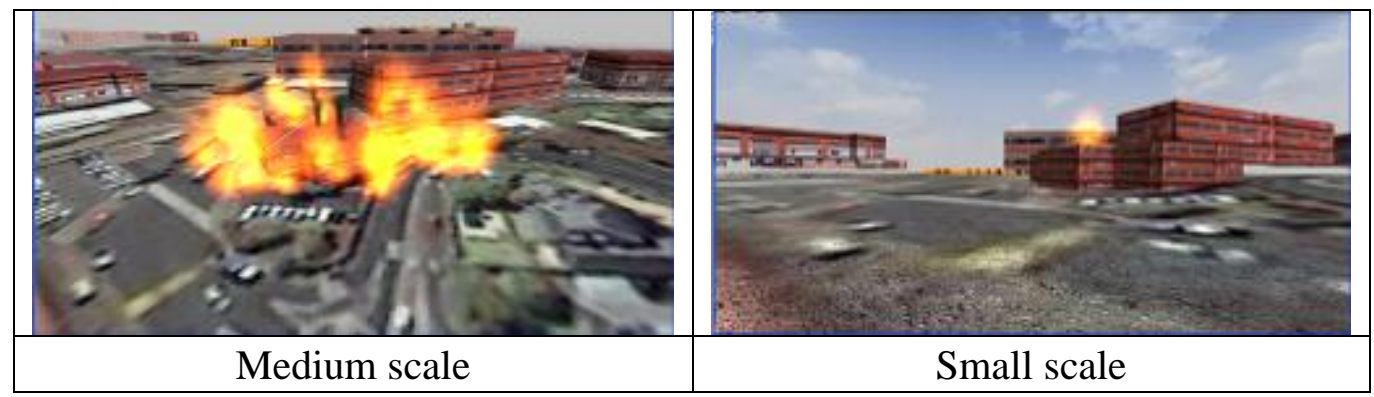

Figure 3.31: Scale of incidents

Incident status changes with time. For example, a medium scale fire incident can become larger or smaller if affected by other environmental factors, such as strong wind or storm. Adjusting the parameter "LifetimevarianceMS" can change the status of incidents.

On the one hand, real-time incident information can be used for monitoring 
instantaneous situations in the area of interest. First responders in the real emergency situation can rapidly take measures to deal with the incident when acquiring the latest information about incident locations and status. Regular updating of information, such as changing status or extending of the affected area, is useful for directing the responders to take alternative strategies. On the other hand, the incident simulation in the virtual environment can also be utilised for emergency training. The incident scenario can provide trainees with a highly authentic experience that is safe and cost effective. Trainees can therefore conduct real-life activities, such as searching for the incident location, rescuing and evacuating the casualties, and controlling or eliminating the incidents. The interaction between the users and the virtual environment is based on situations from the real world. This will be discussed further in Chapter 4.

\subsection{Spatial Interpolation for Real-Time Data}

Emergency managers or decision makers often require spatially continuous data over an area of interest to make effective and informed decisions. However, data is not usually readily available, and is often difficult or expensive to acquire. Despite this, if data at adjacent locations is available, it is still valid to estimate spatial variables for the un-sampled locations using spatial interpolation techniques. Interpolation is a widely used method for geospatial data, such as elevation, precipitation, and soil condition. In this research, it is also necessary to interpolate information in the CVE. Weather data, for example, is only available at weather stations, but the focused location in the CVE may be between stations.

Several methods have been developed for spatial interpolation, such as inverse distance weighting (IDW) (Shepard, 1968), Thiessen polygons interpolation (Tatalovich, 2005), Thin Plate Splines (Wahba, 1990), and Kriging interpolation (Bayraktar \& Turalioglu, 2005). In this research, considering the operational capability of the system and data accuracy, two algorithms were incorporated into 
SIEVE-VTS: IDW and Thiessen polygons interpolation.

IDW interpolation is one of the most widely used methods for interpolating dispersed points. It is based on the theory of spatial dependence, i.e.: "the propensity for nearby locations to influence each other and to possess similar attributes" (Goodchild, 1992, p. 33). Each dispersed point is assigned a weighted variable, which decreases when the distance to the interpolation point increases. Finally the interpolated value can be represented as a weighted mean value of the dispersed points. In SIEVE-VTS, the IDW algorithm is designed as follows:

Algorithm 3.5: IDW interpolation using Shepard's method

Input: intNum: the number of scattered points used for interpolation;

scPoints $[N]$ : array of all scattered points in the area;

intPoint: the un-sampled point at the location( $\mathrm{x}, \mathrm{y}, \mathrm{z})$.

Output: $v$ : the interpolated value.

5.1. if int Num $<=N$ then

5.2. SortScPoints(scPoints, inPoint)

/* Sort the scattered points in decent order based on the distance to inPoint */

5.3. if $\operatorname{SortOK}()$ then $/ *$ Sorting is satisfied */

5.4. While $k<$ intNum Do

5.5. $\quad w=1 /$ Pow (Distance(scPoints $[k]$, intPoints), 2)

5.6. $\quad v=v+w * s c P o i n t[k] . v a l u e$

5.7. $s=s+w$

5.8. $++k$

5.9. $v=v / s$

5.10. else

5.11. Error()

This algorithm is adapted from "Shepard's method" (Shepard, 1968). In order to reduce the impact of the points that are distant from the interpolation point, all the points are sorted in ascending order, based on their distances to the interpolation point intNum is defined by users as less than $N$, indicating that only portions of the dispersed data are selected for interpolation (line 5.1 - 5.2). The fundamental principle is:

$$
F(x, y)=\sum_{i=1}^{n} w_{i} f_{i}
$$


in which $n$ is the number of dispersed points used for interpolation, $f_{i}$ is the function value at one dispersed point (e.g. the observed temperature from a weather station), and $w_{i}$ is the weight function assigned to each point. The basic form of the weight function is:

$W_{i}=h_{i}^{-p} / \sum_{j=1}^{n} h_{j}^{-p}$

where $p$ is a random positive real number named weighting exponent and is defaulted to $2, h_{i}$ is the distance from the dispersed point to the interpolation point or

$h_{i}=\sqrt{\left(x-x_{i}\right)^{2}+\left(y-y_{i}\right)^{2}}$,

where $(x, y)$ are the coordinates of the interpolation point and $\left(x_{i}, y_{i}\right)$ are the coordinates of each dispersed point. The weight function ranges from a value approaching 0 to a value of infinity as the distance from the scattered point decreases. Based on this, the algorithm calculates the final value of the interpolated point (line $5.3-5.9)$

Another interpolation method uses Thiessen polygon or Voronoi diagram (Aurenhammer, 1991), which was first proposed by Alfred H. Thiessen to implement weather predictions using precipitation data from scattered weather stations (Thiessen, 1911). This method is based on the assumption that the unknown value of a point is approximately equal to the value of the nearest sampled point. 


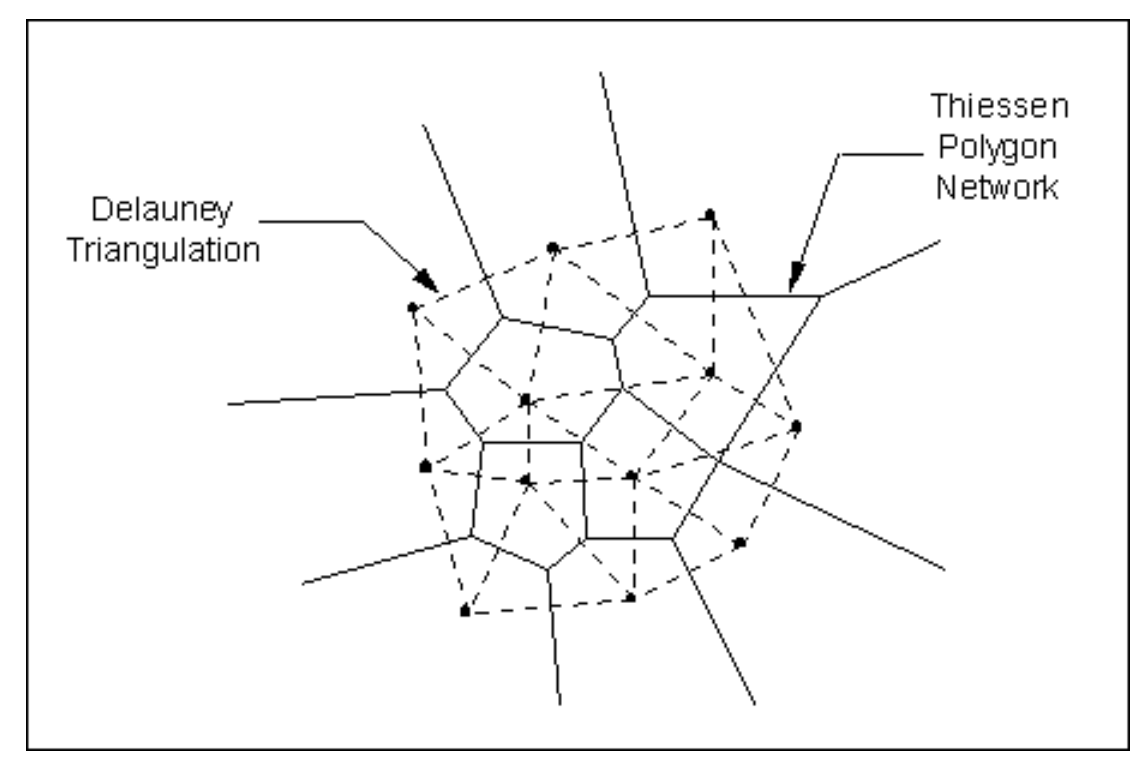

Figure 3.32: Delaunay triangulation and corresponding Thiessen polygon network for a set of scattered points (Aquaveo, LLC., 2010)

Figure 3.32 is an example of a Thiessen polygon network. It can be constructed from the Delaunay triangulation of a scattered point set. Drawing the perpendicular bisector to each side line of the triangles forms the Thiessen polygons, and the value of the scattered point can be used to represent the value of the polygon. Since any location within a particular polygon is nearer to that polygon's point than to any other point, the interpolated value of a location within any polygon equals to the polygon's value. Thiessen polygons assign an "influence area" around each member of a set of points, such that any location within a particular polygon has the same value (Heywood, Cornelius, \& Carver, 1998). The algorithm of Thiessen polygons is incorporated into SIEVE-VTS. 
Algorithm 3.6: Thiessen polygons interpolation

Input: $s c$ Points[N]: array of all scattered points in the area; intPoint: the unsampled point with the location $(\mathrm{x}, \mathrm{y}, \mathrm{z})$.

Output: $v$ : the interpolated value.

6.1 $d t=$ BuildDelaunay (scPoints) $\quad / *$ build Delaunay triangulation for scattered points*/

6.2 For $\left(\right.$ eit $=d t . e d g e s \_b e g i n() ; e i t !=d t . e d g e s \_e n d() ;$ eit ++$)$

6.3\{

6.4 $\quad o b=d t$.dual (eit) $\quad / *$ find the dual graph of the Delaunay triangulation */

6.5 if (ob.type = segment) then $/ *$ draw segment if it is segment*/

6.6 drawsegment()

6.7 else if $($ ob.type $=$ ray $) \quad$ then $/ *$ draw ray if it is ray*/

6.8 $\quad \operatorname{drawRay}()$

$6.9\}$

6.10 For $(i=0 ; i<=$ PolyNum; $i++)$

6.11 \{

6.12 if pointinpoly(intpoint, poly[i]) then $/ *$ determine which polygon intPoint is in*/

6.13 $\quad v \leftarrow$ poly[i].value $\quad / *$ assign the value of polygon to intPoint*/

$6.14\}$

A classic algorithm called convex hull interpolation method is utilised to generate Delaunay triangulation network (line 6.1) (Tsai, 1993). Thiessen polygons are built by drawing the dual graph of Delaunay triangulation (line $6.2-6.9$ ). Finally, the algorithm determines which Theissen polygon the interpolated point is in, and the polygon's value will be assigned to that point (line $6.10-6.14$ ).

The IDW and Thiessen polygon interpolation algorithms have been incorporated into SIEVE-VTS. When the application starts, users can select either method depending on data availability and interpolation preference. When the dataset is large enough, it is more appropriate to choose the IDW method; when the dataset is relatively small, it is more suitable to use the Thiessen Polygon. Data types that can be interpolated include most weather information such as temperature, precipitation, atmospheric pressure, and part of the oceanographic data. Some data, however, like AIS data and incident locations and status cannot be interpolated because such data is not spatially continuous and is not autocorrelation. 


\subsection{Summary}

In this chapter, a prototype system, SIEVE-VTS was proposed in order to realise the goal of utilising real-time data to enhance the authenticity of the virtual environment. The design and implementation of SIEVE-VTS were described: it is composed of a real-time data collector, a data translator, a data simulator as well as an event-based geo-database. It enables users to integrate marine-related real-time information from online content providers and mobile devices, translate and visualise it in a spatially referenced virtual environment where one or many users can explore the incident scenarios.

Three components of SIEVE-VTS were explained in detail. RTDCollector was designed for retrieving real-time data from information providers to SIEVE-VTS, and for storing data into MySQL. Four representative data types were selected and discussed as examples, based on the data availability and usage: real-time weather, oceanographic information, shipping, and incident locations and status. In order to convert abstract data in the real world to visualised models in the virtual environment, RTDTranslator was responsible for modelling the projection from original parameters to visual results in data formats which can be supported by SIEVE-VTS. RTDSimulator, as the visual simulation component, was also developed for users to visualise the final result in different data types. Spatial interpolation was also applied in this system to address the issues of collecting information in an area of interest where limited real-time data is available.

The system is ideal for emergency management, especially for emergency monitoring and training purposes. SIEVE-VTS has the advantage of being capable of capturing real-time data and visualising it simultaneously. Using data which is based on real conditions can enhance users' immersive experience and engenders feelings of personally being on the scene. Although this can partly be achieved in other systems such as VBS2 or ADMS (Section 2.3.2) using historical or hypothetic data, SIEVE-VTS 
can better utilise real-time data. This is particularly important in emergency monitoring, and can also be advantageous in training. More details are illustrated in Section 5.4. Another advantage of SIEVE-VTS is that it manipulates spatial information more efficiently. Using the embedded $M y S Q L$ database and its spatial extensions, geospatial data can be effectively managed during the run time of the simulation. In addition, the system is easy to extend due to the flexibility of TGEA, which supports integration of third party libraries. New functions can be integrated into the software via a common interface without the need to connect to other software.

The potential long-term users are not confined to defence organisations but can also include other emergency management agencies and government administrations. This group, composed of both public and private sector users, includes the police force, fire department, emergency medical technicians, local government and health care organisations. Modification of the SIEVE-VTS prototype can easily enable the current system to be implemented in new fields. For example, a fire fighting agency can utilise this system for emergency planning, command and control, fire discovery, and civilian evacuation. The emergency medical sectors can use it for virtual training purposes including: rescuing casualties in danger, instrument manipulation, navigating through congested traffic, and team collaboration. Users from the general public could adopt it as a education and training tool to improve their decision making ability in an emergency situation.

Despite these advantages, some issues still warrant further consideration:

1. Data quality: Data accuracy cannot be guaranteed since real-time data is collected from different sources which apply different standards. Some data cannot be delivered to the end users on time, which might invalidate the timeliness of the data. Data completeness needs to be enhanced by incorporating more data types into SIEVE-VTS 
2. Polygon limitation on objects: There is a 10,000 polygon limit on each.$d t s$ format object. Therefore, using alternative methods to improve both the quality of the model and the system's performance is useful.

3. Synchronising issue: When users want to view the same scenario in multiple environments and collaborate on different platforms, synchronising the real-time data is challenging. A method using High Level Architecture (HLA) is proposed as future research in Section 6.3.3. 


\section{Chapter 4 Emergency Scenario Simulation Using SIEVE-VTS}

\subsection{Introduction}

As introduced in Chapter 2, emergency management involves reducing risks, preparing resources to respond to hazards, limiting further damage, and returning as closely as possible to the state before the emergency occurs. Simulating previous incidents using historical data, and predicting potential hazards based on current conditions can help decision makers and first responders improve emergency management.

A key objective of this research was to assist emergency management through integration and visualisation of real-time data (weather, oceanographic data, traffic condition, incident location and status), as detailed in Chapter 3. A further objective is to develop a system and an updated user interface that is capable of simulating a wide variety of incidents. This chapter describes the related process and outcome.

In order to appropriately simulate dynamic incident situations, the concept of scenario is introduced in this chapter. In the context of emergency management, scenarios are realistic stories that describe emergency incidents that may happen to people, infrastructure, or the natural environment under a given set of conditions. Such scenarios may be taken from significant events that have occurred in the past, or scenes that could be forecasted in the future. The scenario can be elaborated in terms of hazard, vulnerability, impact and emergency response. Recent studies have used scenarios to carry out simulation in many areas. For example, the U.S. Homeland Security Council (HSC) and Department of Homeland Security (DHS) developed 15 all-hazard planning scenarios during national, federal, state, and local homeland security preparedness activities (U.S. Department of Homeland Security, 2006). In 
another example, a Swiss Virtual Reality (VR) laboratory dealt with practical realisation of an immersive VR training system supporting a broad spectrum of scenarios featuring interactive virtual humans (Ponder et al., 2003). Scenarios can be deployed in numerous types of emergency incidents, including medical emergencies, criminal activities, fires, floods, chemical, radioactive or biological spills, and bomb threats.

Realistic and abstract 3D visualisation of scenarios in a CVE have assisted the communication of processes and outcomes and enhanced emergency management engagements and decision making. "VR offers the potential to create systematic human testing, training, and treatment environments that allow the precise control of complex, immersive, dynamic three-dimensional (3D) stimulus presentations, within which sophisticated interactions, behavioural tracking and performance recording is possible" (Rizzo \& Kim, 2005, p. 1). The advantage of simulating emergency scenarios in $3 \mathrm{D}$ environments is that multiple and complex events can be viewed simultaneously, and it provides a near-true environment within which the users may meet similar situations in the real world.

One purpose of using scenarios for emergency management is to carry out training, and to test preparedness measures and response plans. As emergency management becomes more professional, users consider it necessary to make training lively, realistic and relevant to actual crises. Based on this, emergency simulation often requires more functionalities than simply visualisation, such as interactivity, collaboration, review and analysis.

Another purpose is to use SIEVE-VTS during real-time situation monitoring. Since SIEVE-VTS collects and integrates real-time data, the system can be utilised for monitoring real situations in certain areas. Incidents are recorded, modelled and simulated as they occur. Analysis and replay after the incidents can be valuable for planning for future events and also as part of emergency training. 
The general methodologies for simulating incident scenarios are outlined in this chapter. Following this, a case study on the selected area - Sydney Harbour is presented.

\subsection{Simulation Enhancement}

Conversion of basic environmental phenomena and emergency incidents to SIEVE-VTS representation has been detailed in Chapter 3. Virtual scenario simulation often draws on a wider variety of functionality than standard incident types and requires an extra level of processing to enhance the capability of the system. One enhancement is virtual environment interactivity, which focuses on the mutual effects between users and the virtual environment. Another enhancement enables users to collaborate remotely online, using SIEVE-VTS as a CVE. In addition, the scenarios need to be recorded for analysis and evaluation after the simulations.

\subsubsection{Interactivity}

In SIEVE-VTS, interactivity refers to the mutual effects between users and the virtual environment, as well as those between virtual objects in the environment. On the one hand, such interactivity is supported by the physical system, including rigid and soft body dynamics, fluid dynamics, collision detection, and kinematics. On the other hand, it is based on the game AI, which is often built with a broad set of algorithms by applying techniques from control theory, robotics, computer graphics and computer science in general. Interactivity plays a significant role in making the simulations appear more real to the observer, and helping effective decision making under particular circumstances. 


\begin{tabular}{|l|l|l|l|l|l|l|}
\hline active & Avatar & AI robots & Weather & $\begin{array}{l}\text { Ocean } \\
\text { Information }\end{array}$ & AIS & Incident \\
\hline Avatar & $\begin{array}{l}\text { Various } \\
\text { interaction }\end{array}$ & $\begin{array}{l}\text { Various } \\
\text { interaction }\end{array}$ & $\begin{array}{l}\text { Decision } \\
\text { making }\end{array}$ & $\begin{array}{l}\text { Decision } \\
\text { making }\end{array}$ & N/A & $\begin{array}{l}\text { Hurt or } \\
\text { kill }\end{array}$ \\
\hline AI robots & $\begin{array}{l}\text { Various } \\
\text { interaction }\end{array}$ & $\begin{array}{l}\text { Various } \\
\text { interaction }\end{array}$ & $\begin{array}{l}\text { Decision } \\
\text { making }\end{array}$ & $\begin{array}{l}\text { Decision } \\
\text { making }\end{array}$ & N/A & $\begin{array}{l}\text { Hurt or } \\
\text { kill }\end{array}$ \\
\hline Weather & N/A & N/A & N/A & N/A & N/A & N/A \\
\hline $\begin{array}{l}\text { Ocean } \\
\text { Information }\end{array}$ & N/A & N/A & N/A & N/A & N/A & N/A \\
\hline AIS & Control & Control & Navigation & Navigation & Collision & Damage \\
\hline Incidents & Control & Control & $\begin{array}{l}\text { Intensify } \\
\text { or weaken }\end{array}$ & N/A & N/A & N/A \\
\hline
\end{tabular}

Table 4.1: Interactivity of virtual objects in SIEVE-VTS

Table 4.1 summarises the interactivities of virtual objects in the SIEVE-VTS. Avatars can collaborate with other users through the network or interact with non-players by talking, attacking, and pursing. Meanwhile, avatars and AI robots can control vehicles, such as cars and ships by getting on or off, and also interact with incidents. For example, some AI players can set fire to buildings while other players, such as emergency responders, can extinguish it (Figure 4.1). 


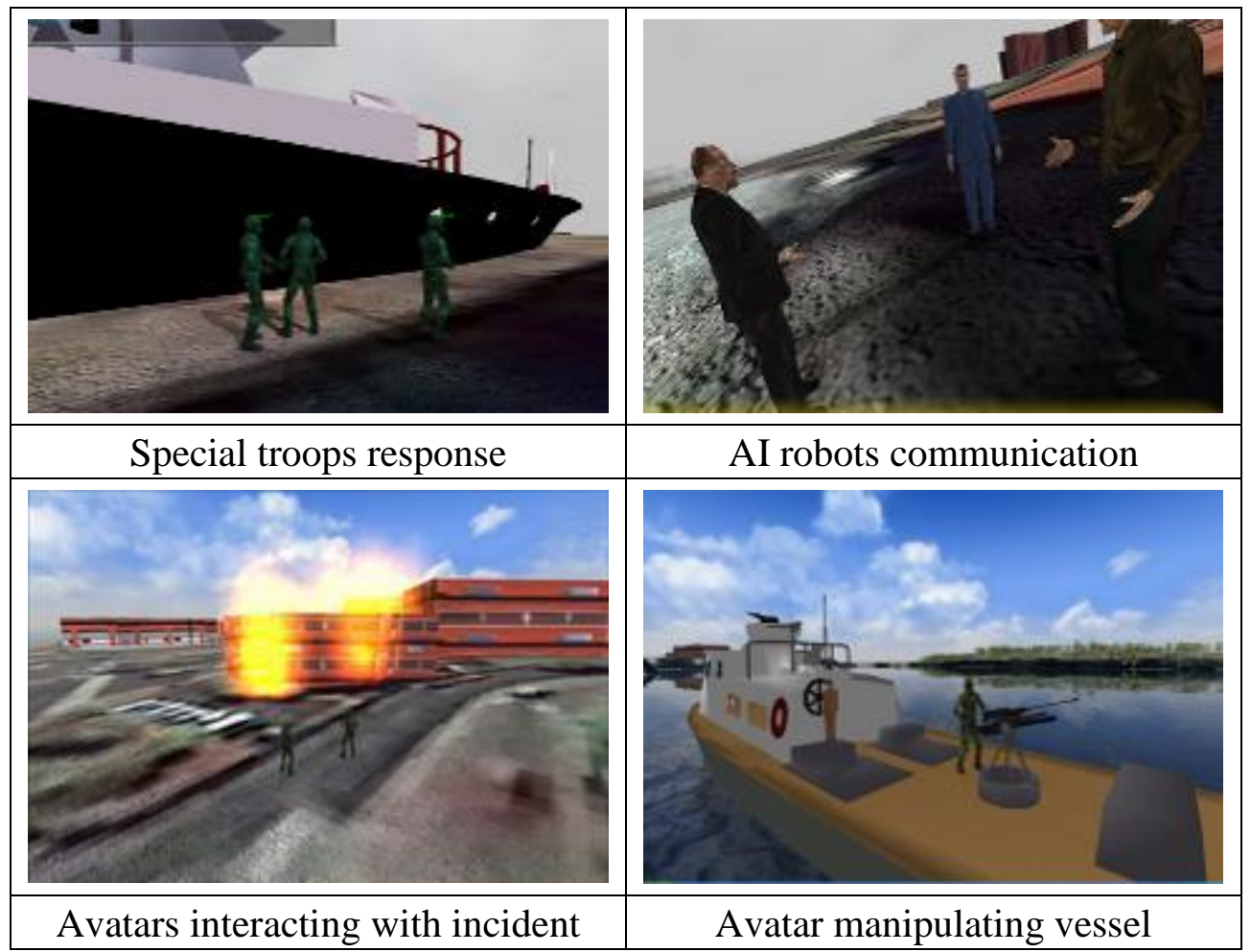

Figure 4.1: Interactivity examples

Weather and oceanographic information may have impacts on the behaviour of avatars, vehicles, ships and incidents. For example, weather information such as changes in visibility or precipitation, can affect avatars' and AI players' speed, navigation and decision making capabilities. Incidents, such as fire, can be extended or extinguished by the changing precipitation and wind. Similar interactivity applies to oceanographic information whereby a variation in water level may warn vessels of the possibility of running onto rocks, and the draught of the vessel may prevent it moving into certain areas. Incidents, such as explosions or fire, can cause damage to buildings, humans, and other ground objects. Any harm to a player can be highlighted with an indicator showing the health condition.

\subsubsection{Collaboration via network}

Incident scenarios cannot only be displayed in one instance of CVE, but can also be viewed and shared between multiple clients. In emergency management, it is important for participants to work collaboratively, and it would be an advantage if 
SIEVE-VTS can support multiple users' collaboration in the virtual environment. For example, in a virtual training situation, trainees are able to communicate with each other in order to cooperate during a rescue mission, and in an emergency response, dispatchers in the monitoring centre can exchange ideas with each other on the latest incident status in order to deliver optimal instructions to the first responders. In this way, geographically isolated users can log into one common virtual space, where each user is assigned an avatar and remotely collaborates with others.

The networking capability is inherently supported by TGEA. It implements a common client-server architecture and attempts to manage three fundamental issues of network simulation programming: limited bandwidth, packet loss, and latency (GarageGames, 2010a). In order to improve efficiency, the server and client in TGEA each has a distinct role. Additionally, the server uses a variety of techniques to limit the amount of data transmission between itself and the clients, thus reducing the network bandwidth requirement for a simulation. The engine utilises efficient game architecture and exceptional network codes, with the practical simultaneous multiplayer limit being as high as 128 players, which is adequate for collaborative simulation of all but very major emergency incidents.

Based on the network capabilities of TGEA, SIEVE-VTS provides the following functions: 1) an updated user interface indicating the scenario name, the number of clients, and other information, which can be used for multiple users to join one virtual scenario through a Local Area Network (LAN) (Figure 4.2) or over the Internet; 2) different roles are assigned to users based on their professions and tasks when they join the server. Different character models and behaviours are connected with the roles. 


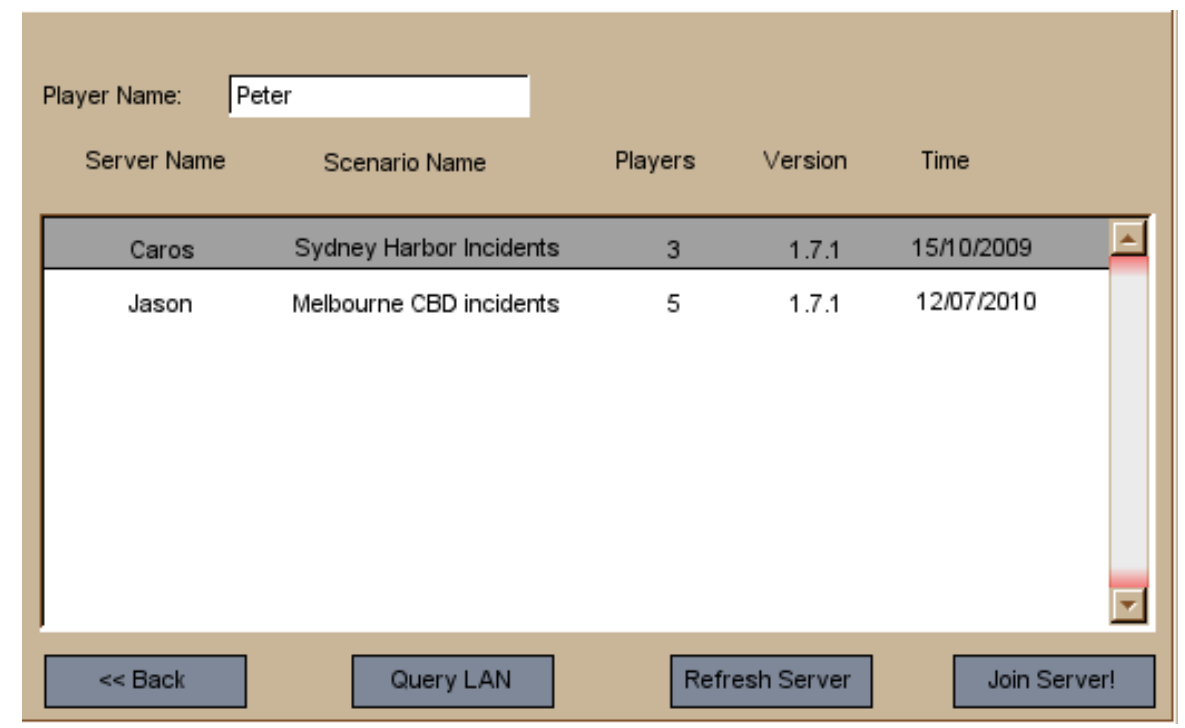

Figure 4.2: GUI of network collaboration

In a complete virtual training system, voice communications and broadcast messaging are usually integrated and operated across the network. Trainees can communicate with each other to share the emergency information in the virtual environment. Instructors can direct the trainees and instruct them with right orders and correct the wrong measures. Currently these functions are not implemented in SIEVE-VTS, but this would be an ideal direction in the future work.

\subsubsection{After Action Review - recording and reviewing}

Evaluation and discussion of the emergency scenario is another important component of emergency management. As described in Chapter 2, trainees are usually guided by facilitators to examine and discuss their actions taken during emergency training, and evaluate their performance after responding to the incidents. Simulating the scenario in SIEVE-VTS is useful but not adequate, new functions of replaying and analysing the emergency scenarios need to be implemented to help the users summarise their experiences and acquire new knowledge.

After Action Review (AAR) is a common solution for this requirement. It refers to a professional discussion of an event, which focuses on performance standards, and 
enables professionals and colleagues with similar interests to discover what happened, why it happened, and how to sustain strengths and improve weaknesses (U.S. Agency for International Development, 2006).

TGEA supports automatic recording contents in a run-time scenario. Based on this, an all-in-one AAR tool with the capability of recording and replaying emergency scenarios is implemented in SIEVE-VTS. It records scenarios via the built-in "NetConnection" class, which stores data stream in a local file under the game engine. When SIEVE-VTS replays the scenario, it directly reads the logged data through a user interface (Figure 4.3). The AAR tool has the capability of marking events during the exercise, storing the events after the exercise and replaying these records in the review mode (Figure 4.4). It also supports multiple view modes during the AAR, including:

- $2 \mathrm{D}$ view - This is the traditional plane view that is effective when demonstrating the overall situation of the focused area.

- $3 D$ fly mode - Users can fly through the 3D virtual environment using the free camera view.

- Entity view - It displays what a selected entity (such as enemy or civilian) sees, including the effects of head turning and posture changes, and the attribute information of the entity itself.

- Event data collection and display - AAR tool has the ability to track an event, including its appearance, disappearance, movement, and other changes. 


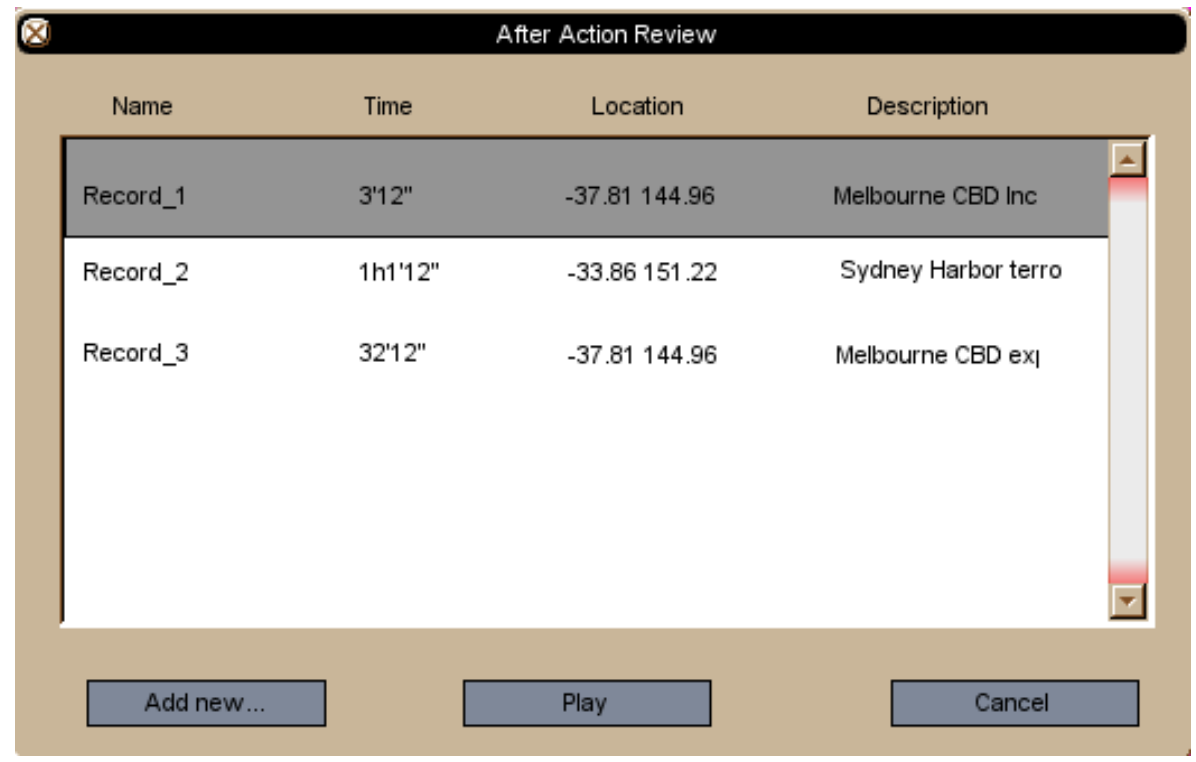

Figure 4.3: After Action Review in SIEVE-VTS: log in panel

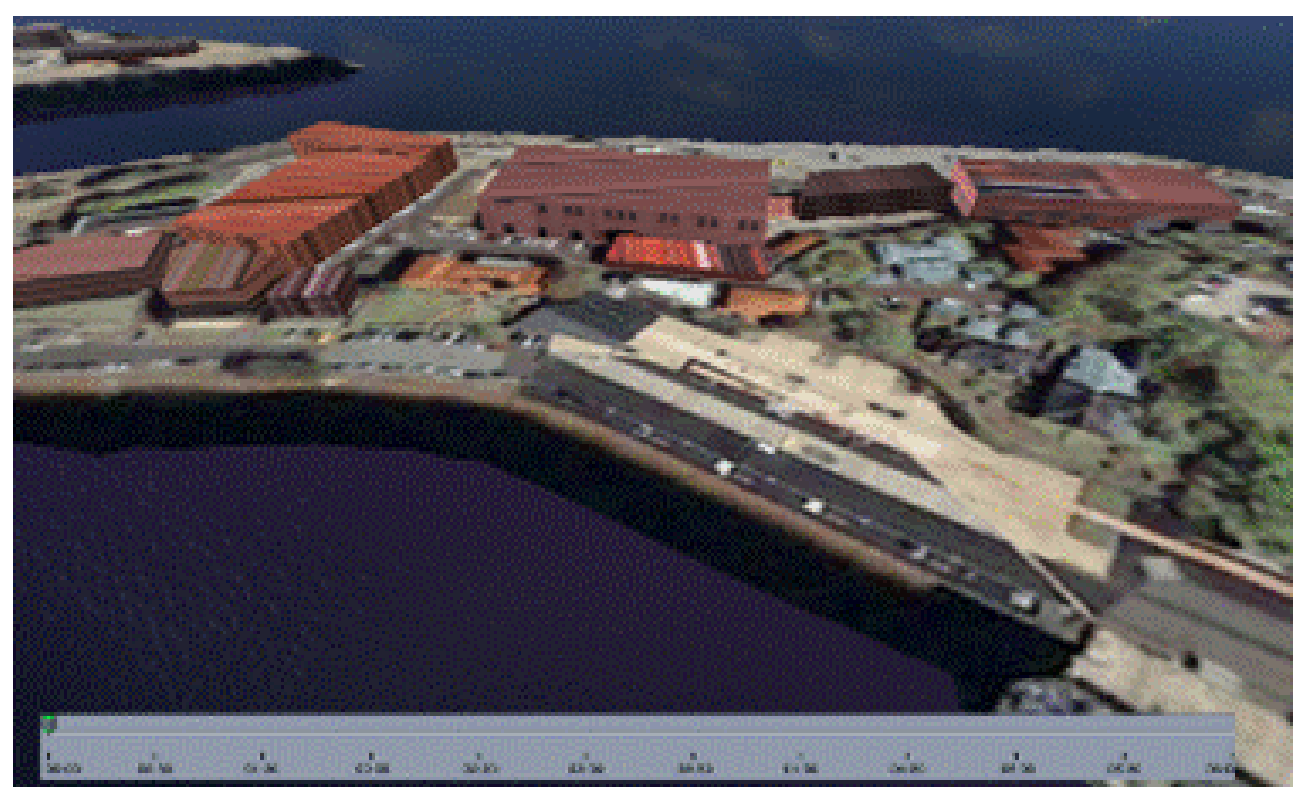

Figure 4.4: After Action Review in SIEVE-VTS: timeline control

During a simulation, the recording function can be started using the "start_recording" function, and ceased by the "end_recording" function. After the simulation, a facilitator can play back the recorded scenarios using the AAR tool, while trainees can select the recording options when logging into SIEVE-VTS, explore the historical scenarios from the various viewing modes, and analyse their training performance. In SIEVE-VTS, AAR is useful in that it provides users with a common understanding of 
the situation and its reasons, helps users identify strengths and weaknesses, and improves their performances in future tasks.

This section described new functions incorporated in SIEVE-VTS, including simulation interactivity, network-based collaboration and AAR. These enhancements were inherently supported by existing features of $T G E A$, and appropriate updates and improvements were achieved based on these features (Table 4.2).

\begin{tabular}{|l|l|l|}
\hline $\begin{array}{l}\text { Simulation } \\
\text { Enhancement }\end{array}$ & Existing Feature in TGEA & $\begin{array}{l}\text { Extra Work in This } \\
\text { Research }\end{array}$ \\
\hline Interactivity & Physical engine and game AI & $\begin{array}{l}\text { Designing and simulating } \\
\text { interactions between } \\
\text { various virtual objects }\end{array}$ \\
\hline $\begin{array}{l}\text { Collaboration } \\
\text { via network }\end{array}$ & $\begin{array}{l}\text { Local network connection and } \\
\text { client-server architecture }\end{array}$ & $\begin{array}{l}\text { Designing log-in user } \\
\text { interface and role } \\
\text { assigning }\end{array}$ \\
\hline AAR & $\begin{array}{l}\text { Recording and replaying } \\
\text { functions }\end{array}$ & $\begin{array}{l}\text { User interface for selecting } \\
\text { the scenario, procedure } \\
\text { control of the AAR }\end{array}$ \\
\hline
\end{tabular}

Table 4.2: SIEVE-VTS enhancement for scenario simulation

\subsection{Case Study}

As described in Section 2.1, emergency management consists of several phases and associated activities, of which, emergency training and monitoring are the focus in this research. Training involves gathering users in a simulated condition and executing a series of tasks that could potentially happen in a real emergency event. Monitoring also involves multiple users but decision making based on real event is required. For the case study, a terrorist attack was selected as the possible event, because it included weather and oceanographic changes, and a range of different incidents. A focus area within Sydney Harbour was selected to test the simulation methodology in the context of emergency management. 


\subsubsection{Focus area}

The area of interest for simulating incident scenarios is located in Garden Island and surroundings within Sydney Harbour (Figure 4.5). Specifically, it is a $2.5 \mathrm{~km} \times 2.5$ $\mathrm{km}$ square area with the centre located at $(33.865 \mathrm{~S}, 151.227 \mathrm{E})$. On the western side lies the Royal Botanic Gardens and Sydney Opera House, which are adjacent to the Sydney CBD; on the northern and eastern sides is Port Jackson, which extends to Pacific Ocean; on the southern side is Potts Point suburb which connects the south Sydney area. Garden Island has been the site of Fleet Base East of the Royal Australian Navy $(R A N)$ since 1980. The area has a climate with warm summers and mild winters, with the average temperature ranging from 13.8 to 21.7 degrees. The average annual rainfall is $1,217 \mathrm{~mm}$, falling on average 138 days a year. Ocean water level is recorded as ranging from $-1.1 \mathrm{~m}$ to $1.47 \mathrm{~m}$ without distinct change in recent years (BOM, 2010a).
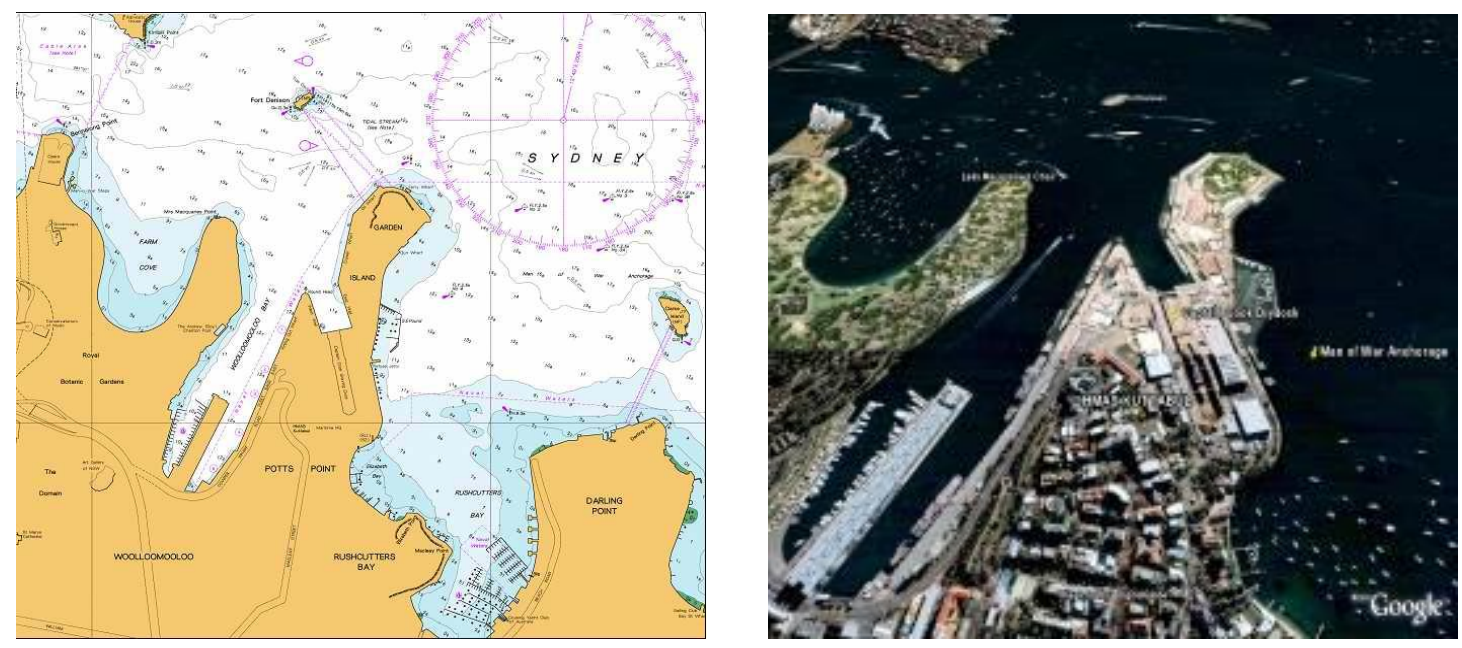

Figure 4.5: Research area - Sydney Harbour

The main reason for selecting this area for simulating emergency scenarios is that: it is a military stronghold and significant national defence base. Garden Island is the primary east coast naval base of the RAN. HMAS Kuttabul, the RAN's administrative and logistics support institute for Sydney area is located here. Other facilities include: Captain Cook Graving Dock, the largest graving dock in Australia; Sydney Fleet 
Headquarters, specifically designed to support maritime operations and organised with major divisions for operations planning; and fleet base east, where the Navy vessels are anchored and training services are carried out. Overall, this area is a key harbour and military defence site for the Australian Navy, and is considered a high level security risk for terrorism. The potential emergency management applications, through appropriate scenarios, include assessment of port and harbour vulnerability, design of solutions to the attack of ships and infrastructure, development of force protection concepts and tactics, and implementation of techniques and procedures for offshore asset protection.

\subsubsection{Static object modelling in SIEVE-VTS}

Before simulating incident scenarios in SIEVE-VTS, it is necessary to build the infrastructure of that area by populating the virtual environment with static objects, including terrain, buildings, and vegetation (Figure 4.6). The core technique is to convert these elements into SIEVE-VTS supported formats, which was achieved and automated in a previous $C R C$-SI project (Stock et al., 2008). For the terrain data, a DEM and the corresponding aerial photograph are provided by Defence Science and Technology Organisation (DSTO), at resolutions of $0.5 \mathrm{~m}$ and $0.25 \mathrm{~m}$, respectively. Terrain data is converted from .shp into .atlas format, a standard terrain data format which can be easily transferred into other formats. Surface objects are simply abstracted as points, linking to objects based on the model outputs. Visualisation of an existing tree, for example, uses a point in the underlying vegetation data and links to an appropriate species type. Other models, including buildings, avatars and vehicles, are firstly created manually in .3ds format using 3ds Max (Autodesk, 2010), and then transferred into .dts format using plug-in components such as DTS exporter for $3 d s$ $\operatorname{Max}$ (Dutka, 2010). While the surface layer is mainly sea water, which surrounds half of the research area, the bathymetry of Sydney Harbour is also potentially important and is provided at a resolution of $1.2 \mathrm{~m}$. The combination of bathymetry and terrain data is used to create the $3 \mathrm{D}$ virtual environment of the research area. The integral 
conversion can be automatically achieved by SIEVE-TGEA-Exporter (Figure 4.7).

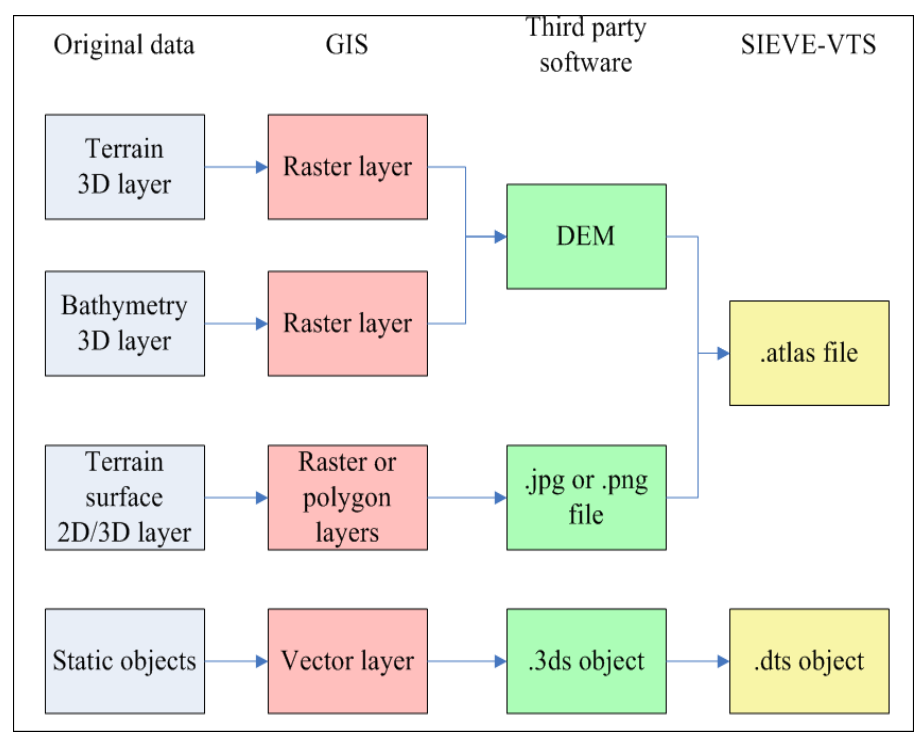

Figure 4.6: Static object conversion in SIEVE-VTS

\section{SIEVE Builder}

Terrain

Object Layer

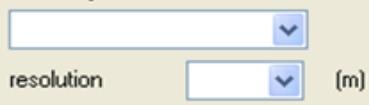

(m)

Ground Texture:

$\square$ current GIS view

Texture Layer:
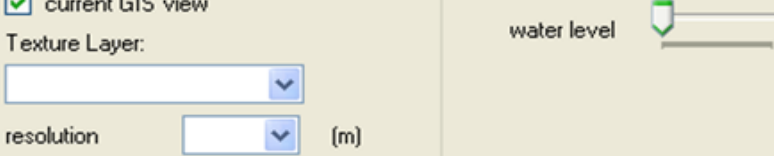

(m)

ipeg $\bigcirc$ png

Paths

SIEVE Viewer path:

Scenario name:

c:/SIEVE
example

Build \>

Figure 4.7: User interface of SIEVE-TGEA-Exporter

\subsubsection{Fully-hypothetic simulation}

The scenario has been shown to be an appropriate tool to plan future actions and promote understanding with respect to potential crisis situations. Incident scenarios are integrated into the SIEVE-VTS to provide simulation functionalities and establish 
the related details. Two types of simulation methods: fully-hypothetic simulation and semi-hypothetic simulation are subsequently discussed and evaluated.

Fully-hypothetic simulation employs hypothetic scenarios. Such scenarios are often designed based on situations that may have occurred previously or have the potential to occur in the future. This method is the traditional way for virtual training and is widely used in current industry designs (Murakami, Sugimoto, \& Ishida, 2005). The hypothetic simulation for the Sydney Harbour terrorism scenario is implemented in the following steps:

\section{Step 1. Scenario briefing}

The scenario provided by DSTO briefly describes a series of incidents caused by a terrorist attack in Garden Island. First of all the following events are introduced:

a. United States Coast Guard (USCG) icebreaker, the Polar Star, saved Australian fishermen when their boat was harassed and sunk by a Japanese whaling "research" ship approximately 200 nautical miles off Jervis Bay on the southern coast of New South Wales.

b. The Polar Star was damaged in the incident and sailed to Port Jackson in Sydney Harbour for urgent repairs at Captain Cook Dock on Garden Island. It was expected to be ready to sail on Monday 12 November.

c. Police reported that a van had been found near a boat ramp near Rushcutters Bay, south east of Garden Island. The van may have been used to transport chemicals. Also, a motor launch was found stolen from a marina in Woolloomooloo Bay.

d. Environmental and IMG protestors planned a protest at Lady Macquarie's Chair on Sunday 11 November to coincide with a 'family day' organised for the Polar Star crew and the people of Eden, the home port of the sunken fishing boat. 
e. On 30 October, a merchant vessel unexpectedly entered Port Jackson without acknowledging any communication requests, nor taking on a pilot. Subsequently, the vessel anchored at the Man of War anchorage off Garden Island.

f. The USS MOBILE BAY was tasked to escort USCG Polar Star out of Sydney Harbour on 12 November. It arrived in Sydney and berthed at Garden Island on 10 November.

g. Before the Polar Star left the dock, a disaffected Master Mariner with possible terrorist connections tricked an outgoing pilot into offering assistance to the stolen motor launch outside the Heads. Then masquerading as the pilot, the Master with an accomplice, got aboard an inbound commercial cargo ship and took control of the bridge. Steaming into Sydney Harbour they changed course near Fort Denison and, with minimal reduction in speed, hit the Captain Cook Dock caisson. This caused substantial and direct damage to adjacent $R A N$ ships tied up seaward of the caisson, and the resultant flooding of the dry-dock caused serious damage to the USCG ship.

Step 2. Events abstracting

In order to simulate the scenarios, the correct locations and time orders of the events need to be clarified. Sydney Harbour maps are used for marking the events and corresponding times. The number depicts the time sequence of the events (Figure $4.8)$. 


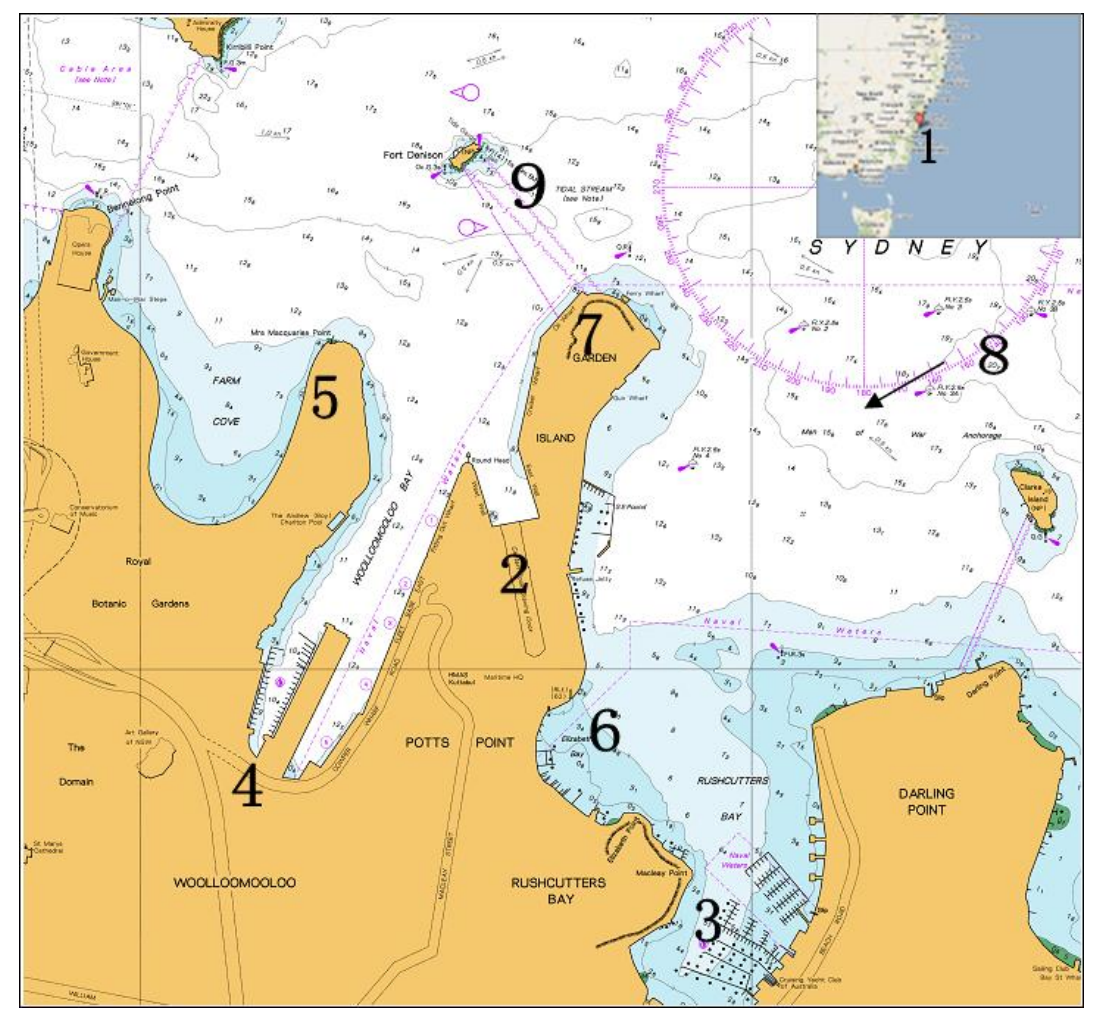

Figure 4.8: Events marking for fully-hypothetic simulation

Step 3. Scene segmentation

Based on the abstracted events, the hypothetic scenario can be divided into multiple scenes. Each scene is assigned a scene ID and a brief description, arranged by time sequence. It may contain one or more events at a certain time and location (Table 4.3). Virtual cameras, which have both dynamic and static positions, are designed and configured in each scene. Users can either explore different viewpoints in one individual scene by changing the camera position, or browse multiple scenes which automatically switch from one to another with time. The combination of the ordered scenes forms the complete emergency scenario. 


\begin{tabular}{|c|c|c|c|}
\hline $\begin{array}{c}\text { Scene } \\
\text { ID }\end{array}$ & Time & Location & Events \\
\hline 1 & 10 October & $\begin{array}{c}200 \text { nautical } \\
\text { miles off Jervis } \\
\text { Bay }\end{array}$ & $\begin{array}{c}\text { Polar Star saved } \\
\text { Australian fishermen }\end{array}$ \\
\hline 2 & 11 October & $\begin{array}{l}\text { Captain Cook } \\
\text { Dock on } \\
\text { Garden Island }\end{array}$ & $\begin{array}{c}\text { Polar Star went for urgent } \\
\text { repairs }\end{array}$ \\
\hline 3 & 12 October & $\begin{array}{c}\text { Rushcutters } \\
\text { Bay }\end{array}$ & $\begin{array}{l}\text { A suspicious van was } \\
\text { found near a boat ramp }\end{array}$ \\
\hline 4 & $\begin{array}{c}10 \\
\text { November }\end{array}$ & $\begin{array}{l}\text { Woolloomooloo } \\
\text { Bay }\end{array}$ & $\begin{array}{l}\text { A motor launch was } \\
\text { stolen }\end{array}$ \\
\hline 5 & 11 November & $\begin{array}{l}\text { Lady } \\
\text { Macquarie's } \\
\text { Chair }\end{array}$ & $\begin{array}{l}\text { IMG members planned a } \\
\text { protest }\end{array}$ \\
\hline 6 & 30 October & $\begin{array}{l}\text { Man of War } \\
\text { anchorage }\end{array}$ & $\begin{array}{c}\text { A merchant vessel } \\
\text { unexpectedly entered Port } \\
\text { Jackson and anchored at } \\
\text { the Man of War } \\
\text { anchorage }\end{array}$ \\
\hline 7 & $\begin{array}{c}10 \\
\text { November }\end{array}$ & Garden Island & $\begin{array}{l}\text { USS MOBILE BAY } \\
\text { arrived in Sydney }\end{array}$ \\
\hline 8 & $\begin{array}{c}12 \\
\text { November }\end{array}$ & $\begin{array}{l}\text { Sydney } \\
\text { Harbour }\end{array}$ & $\begin{array}{c}\text { A disaffected Master } \\
\text { Mariner offered assistance } \\
\text { to the stolen motor }\end{array}$ \\
\hline 9 & $\begin{array}{c}12 \\
\text { November }\end{array}$ & $\begin{array}{l}\text { Fort Denison } \\
\text { and Captain } \\
\text { Cook Dock }\end{array}$ & $\begin{array}{c}\text { The controlled } \\
\text { commercial cargo ship } \\
\text { changed courses and } \\
\text { rammed into Captain } \\
\text { Cook Dock, causing } \\
\text { serious damage }\end{array}$ \\
\hline
\end{tabular}

Table 4.3: Scene segmentation

Step 4. Detailed design

Events in each scene must be narrated in detail by coding in scripts (Figure 4.9). For example, scene 5 describes a protest near Lady Macquarie's Chair, where the main focus is simulating the crowd behaviour. Character models and gestures are predefined, whereby characters are assigned random actions during the simulation. In scene 9, ships, characters and incident models are pre-designed. After the simulation 
starts, the interactions of AI characters and vehicles are created; when the ship is mounted by a marine master, it navigates from one location to another following the established routes. Finally, the explosion and fire incidents in the surrounding area are triggered.

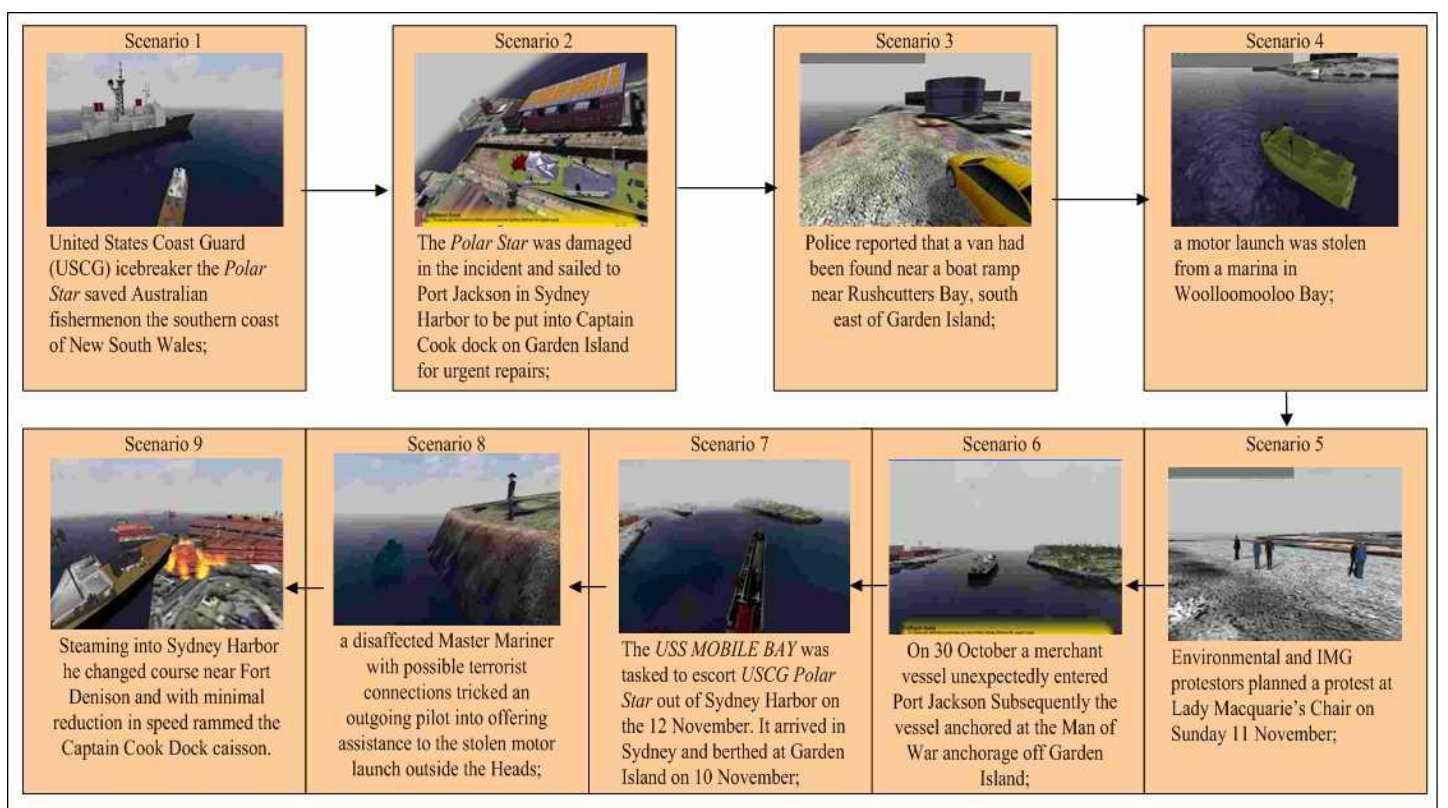

Figure 4.9: Detailed design

Step 5. Ancillary data integration

Ancillary data in a fully-hypothetic simulation include weather, oceanographic and traffic conditions, which are also based on hypothetic information. Weather, tide and swell can be setup using default configurations that are coded in the mission file. Marine traffic is pre-designed in the storyline before the scenario starts. For example, in scene 9, vessels move within the Sydney Harbour area on a scheduled path. When the incidents occur, some of the vessels are damaged by the explosion, while some choose alternative routes to escape the incidents.

\subsubsection{Semi-hypothetic simulation}

In semi-hypothetic simulation, part of the scenario is based on hypothetic situations, while the other part is specifically built from real world situations. In this case, a 
hypothetic storyline is utilised by RTDSimulator for simulating the incident scenario, meanwhile real-time data is also collected by RTDCollector. The simulation was taken on October 11, 2009. Generally there are four steps taken in a semi-hypothetic simulation.

Step 1. Scenario briefing

A terrorist attack at Garden Island is part of a series of fictitious events. The scenario development is as follows:

a. At 11:20 a.m., the USCG Alex Haley was damaged in an incident. It sailed to Port Jackson in Sydney Harbour and settled down in Captain Cook Dock for urgent repairs.

b. At 3:06 p.m., a merchant vessel entered Port Jackson and berthed at the Man of War anchorage off Garden Island. It accidentally hit another cargo vessel nearby. The incident attracted attention from local police and security agencies.

c. At 6:30 p.m., a commercial cargo ship steamed into Sydney Harbour, and changed course from its proposed route and destination near Fort Denison. The marine security sent messages inquiring about the change, but did not get a clear response.

d. At 6:55 p.m., the commercial cargo ship hit the Captain Cook Dock caisson, which caused direct damage to adjacent $R A N$ ships tied up seaward of the caisson. The resultant flooding of the dry-dock caused serious damage to the USCG ship.

e. At 7:10 p.m., emergency responders started taking measures in order to minimise their impact and to rescue victims and evacuate civilians.

f. At 9:00 p.m., through the collaboration of multiple agencies, including fire fighters, police forces, counter-terrorism units, as well as the medical emergency 
department, the incident was gradually controlled, and the victims in the incidents were rescued and sent to hospitals for emergency treatment.

Step 2. Timeline design

Similar to the previous scene segmentation in fully-hypothetic simulation, the whole scenario was divided into several sections based on their time attributes. All the events were distributed and labelled on a timeline, therefore new events could be automatically triggered by time point or other related events in the scenario (Figure 4.10).

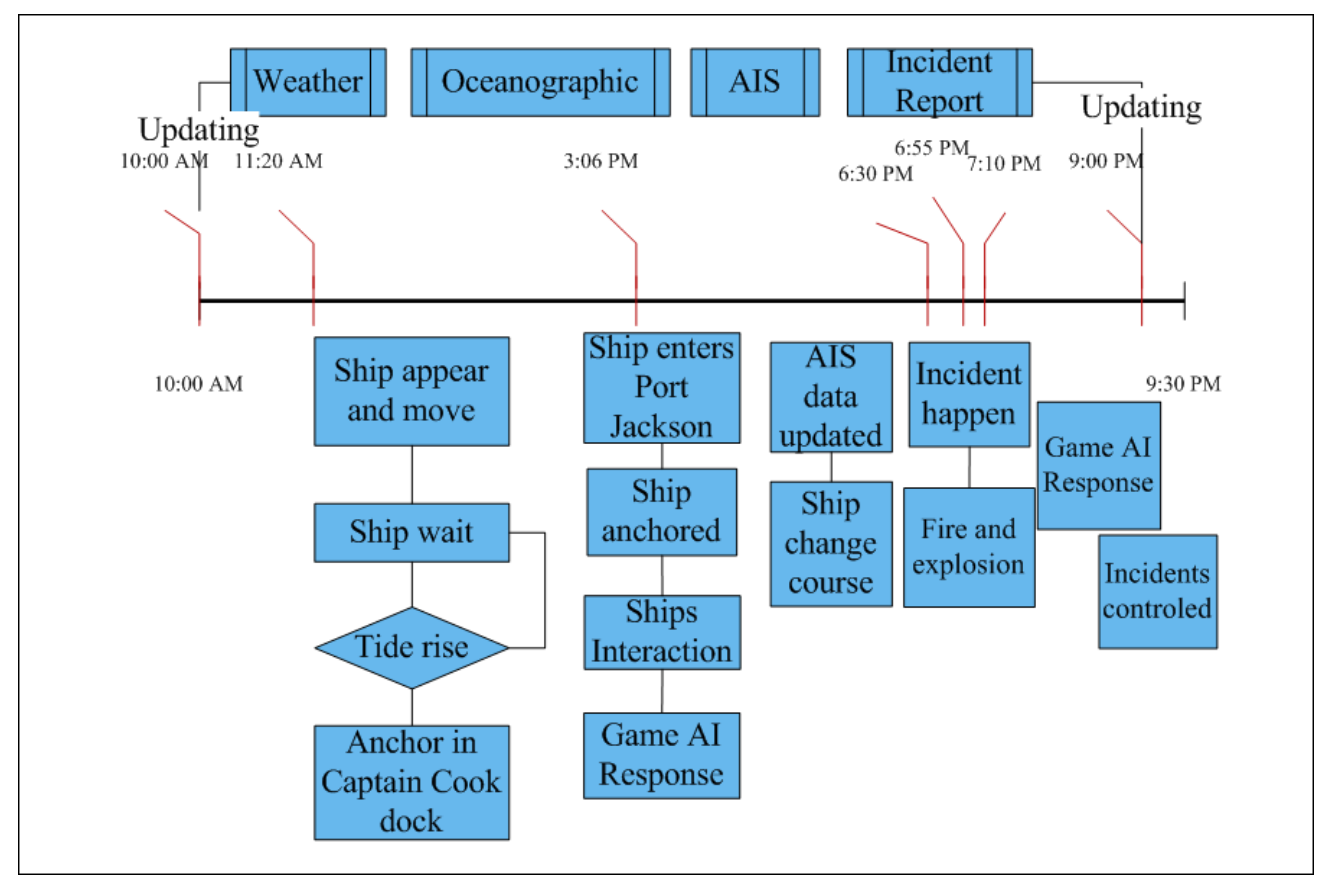

Figure 4.10: Time line design for semi-hypothetic simulation

The real-time data panel was launched in order to build a connection with the online data sources. The RTDCollector monitored the change of data source and made notifications to the RTDSimulator when new data was updated. For example, real-time weather was updated every 5 minutes and rendered in RTDSimulator simultaneously.

Step 3. Detailed scenario design 
1. When time passed 11:20 a.m., the first event of the USCG Alex Haley shipping into Sydney Harbour was triggered, and the route was built from historical AIS data. When the ship reached the nearby area of the port, it stopped momentarily to determine whether it was safe to pass through the water area according to the real-time tide and swell information retrieved from the online content providers. The ship would move through the area when the data indicated that it was safe to do so, otherwise it had to seek an alternative strategy.

2. The next event was triggered by time point 3:06 p.m., when AIS data showed the route of a merchant vessel which entered Port Jackson unexpectedly. Later it moved into a forbidden area and crashed with other vessels. This incident was recorded and sent by a local security guard using iReport. Interactivity was also applied such that the collision of vessels caused damage to both of them; meanwhile, the collision also triggered the subsequent event of police force responding to the incident, in which a game AI routing using $\mathrm{A}^{*}$ algorithm was implemented.

3. The real-time data was regularly updated during the simulation. At time 6:30 p.m., the AIS showed that a commercial cargo ship changed its regulated route and previous destination, which triggered an inquiry from marine security organisation, however, no response was received.

4. Time point 6:55 p.m. triggered a new event that the commercial cargo ship crashed into $R A N$ ships at high speed. Fire and explosions were simulated by the RTDSimulator. The broader environmental impact was that the incidents caused serious damage to nearby buildings and infrastructure. Real-time data about the incidents was reported and updated from mobile devices.

5. When the police force received the incident data, fast response was made at 7:10 p.m. As the real-time data kept updating, some basic environmental conditions were changed: a) it was getting darker since the time was late; b) weather 
changed from clear conditions to cloudy and windy, which extended fires to surrounding areas; c) due to the smoke caused by the fire, visibility was dramatically reduced, which affected the performance of players. An updated AI path finding was also applied with more restrictions such as lower visibility and higher level of danger.

6. By the time of 9:10 p.m., the incidents were gradually under control, as shown from the updated incident location and status report. The scenario was alive until it finished at 9:30 p.m.

\section{Step 4. Scenario packaging}

All the scenarios were recorded into a database and scripted files. The packaging procedure was fast and automatic, the result of which was convenient for AAR or for use as historical data in future training.

When the methods of fully-hypothetic and semi-hypothetic simulation were compared, significant differences were found between them:

- Complexity of scenario creation: In fully-hypothetic simulation, it usually takes several days or even weeks to create a scenario manually, depending on the complexity of the scenario itself. In semi-hypothetic simulation, since it automatically retrieves real-time data from online sources and visualises it as events in the virtual environment, the procedure of designing scenarios is simplified, therefore the complexity of scenario creation is reduced.

- Authenticity: In terms of visual effect, both simulation methods share similar fidelity based on the same rendering and graphic processing system. However, since the semi-hypothetic simulation augments the scenario using real-time data, it can enhance the authenticity by increasing the sense of reality for users.

- Data management: In SIEVE-VTS, fully-hypothetic simulation utilises the 
traditional data file systems to manage scenarios. For example, the scenes, incidents, and traffic route are scripted into mission files of the game engine. The semi-hypothetic simulation stores real-time data into databases, and the data is used to design events such as weather changes and emergency incidents. The advantages of using a database were introduced in Section 3.9.

- Spatial information representation: Fully-hypothetic simulation represents the spatial data using virtual coordinates. This does not necessarily represent the real circumstance or may not be internally consistent. For example, the ships might be collided with each other due to the inconsistency of the tracking data. The semi-hypothetic simulation uses spatial information from real-time world, such as the traffic route of vessels or the locations of the incidents, which reflects the real situation.

\subsection{Summary}

In this chapter, the process and outcome of simulating various events in the virtual environment were illustrated. Firstly, the interactivity, network collaboration and AAR functionalities were enhanced in order to support the advanced virtual simulation. Then a case study was carried out in the Sydney Harbour area in order to demonstrate that SIEVE-VTS is able to simulate incident scenarios for emergency management. Static data including terrain, vegetation, and buildings were generated using SIEVE-TGEA-Exporter before the simulation began, and ancillary data were integrated into the system during the simulation as well. Finally two methods, fully-hypothetic and semi-hypothetic simulations were introduced. It was shown that the semi-hypothetic simulation using real-time data can greatly improve the authenticity and reduce the complexity of scenario creation. 



\section{Chapter 5 System Test and Evaluation}

\subsection{Introduction}

This research involves defining requirements for a collaborative visualisation tool from available literature and building a system to fulfil these requirements. While comprehensive testing of SIEVE-VTS is beyond the resources of this research, two methods, including performance and functional evaluation are used to provide preliminary testing and feedback with respect to these requirements. The feedback provides valuable information relevant to development and formal testing of SIEVE-VTS in the future.

Section 5.2 reports the testing of some functions important to the performance of SIEVE-VTS. This has provided results establishing that:

1. Various types of incident scenarios can be simulated in SIEVE-VTS with acceptable rendering time;

2. Real-time spatial information can be efficiently shared by users at different locations via a network.

In Section 5.3, a demonstration of SIEVE-VTS is carried out to a group of potential users of this system, in order to gain feedback on its key functionalities. This testing demonstrates that:

1. The authenticity of CVE can be highly improved by integrating real-time data;

2. The spatial information and CVE can be closely connected;

3. The system can be utilised as an emergency management tool, with the capabilities of simulating, training and monitoring; 
4. The system can make full use of mashup technology, and extend it from traditional 2D maps to the 3D virtual environment.

The test results and feedback on system performance and functionality are summarised in this chapter. The potential usage of SIEVE-VTS is also further discussed.

\subsection{Performance Evaluation}

The aim of the performance evaluation is to ensure that the suggested system possesses a satisfactory range of performance consistent with the intended objectives of the research. The discussion relating to the performance of SIEVE-VTS is based upon criteria that include its rendering effects and network performance. The server side of the system runs on one PC, while the client side of the system runs on another PC with the same configuration. The specification of the test environment is as follows (Table 5.1):

OS: Windows XP (SP2)

Processor: Intel(R) Core(TM)2 Quad CPU Q6600 @ $2.4 \mathrm{GHz}$

RAM : 3.25 GB

Graphics Card: NVIDIA GeForce 8800GT

Torque Game Engine Advanced Version: 1.7.1

Table 5.1: Performance test specifications

\subsubsection{Rendering performance and scene complexity}

When simulating a large environment such as Sydney Harbour, the issue of computational load becomes a point of interest. Rendering time is based on several factors. In the general case, it can be expressed by the function: $R T=f(S G, R A, H W$, $S T)$, where $S G$ is the scene graph, $R A$ is the rendering actions used to generate a $2 \mathrm{D}$ view, $H W$ is the hardware, and $S T$ is the state of the system (Wimmer \& Wonka, 2003). The function can be simplified by replacing the first parameter $S G$ with viewable 
objects, attributes and geometry, while $R A, H W, S T$ are fixed by the game engine and hardware.

When a large number of entities need to be rendered, the system still completes the rendering but performs at the cost of frame rate. This provides a metric indicative of the system's load. The engine's decline in frame rate, which is symptomatic of a rise in load, is used to indicate how these affect performance. Frame rate, often expressed in frames per second (FPS), is the frequency at which an imaging device generates unique successive images. When the FPS is reduced, users might feel encumbered, which will affect their manipulation and collaboration in the virtual environment.

To check the rendering performance of SIEVE-VTS, FRAPS (Beepa Pty Ltd, 2010) is utilised, as it provides video and screen capturing along with a measurement for 3D game's frame rate. The relationship between FPS and the number of entities is thus revealed (Figure 5.1). Three types of entities are selected for testing, including ship, player, and building. Each type has specific complexities in its geometry (Table 5.2).

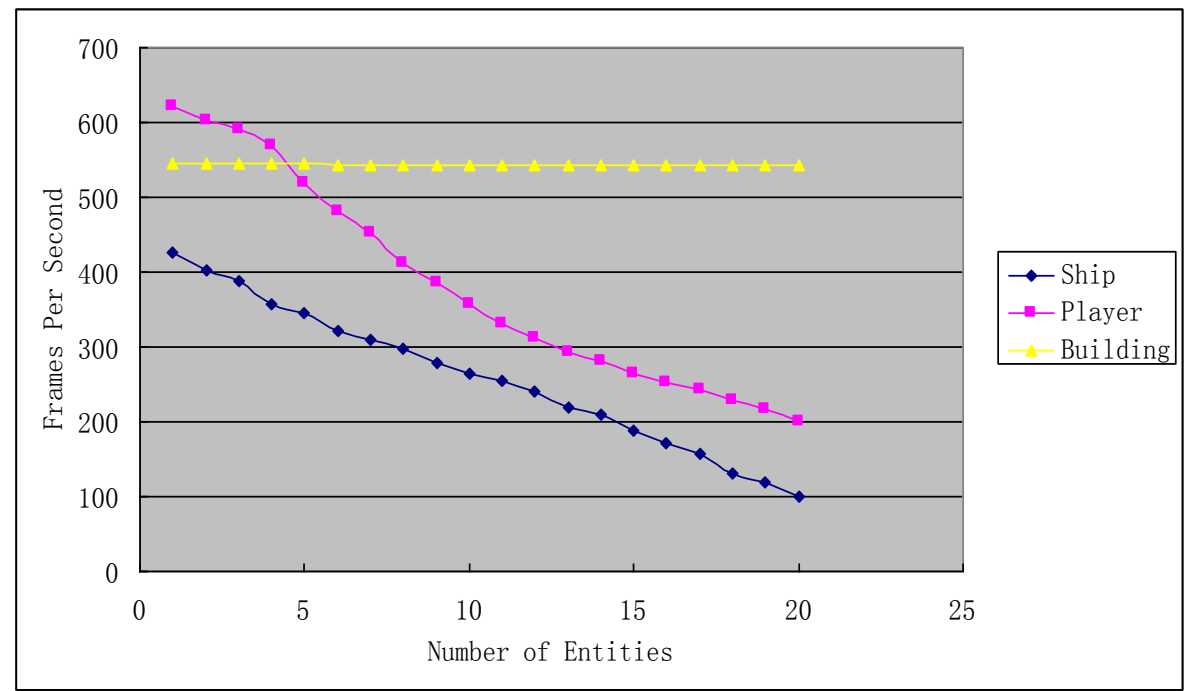

Figure 5.1: FPS and scene complexity 


\begin{tabular}{|c|c|c|c|}
\hline & Vertices & Faces & Model Size \\
\hline Ship & 8,726 & 10,442 & $656 \mathrm{~KB}$ \\
\hline Player & 2,350 & 3,123 & $140 \mathrm{~KB}$ \\
\hline Building & 8 & 12 & $2 \mathrm{~KB}$ \\
\hline
\end{tabular}

Table 5.2: Object complexity

The result shows a clear stair step trend toward reducing the frame rate as the number of entities increase for "ship" and "player", while there is no obvious drop in the case of "building". This is because the complexity of a single building object is relatively low, with only 12 faces; therefore the increasing number of objects does not obviously affect the total scene complexity, thus the frame rate. While in the case of "ship" and "player", increasing the number of objects can dramatically increase the total number of faces. It can be concluded that FPS is reduced with the complexity of scenes, or more specifically, the overall faces of 3D objects.

The FPS is still over 30 fps when tested using more than 1 million faces in the scenario, which can form more than 80,000 buildings, 320 human characters, or 100 ships (various at the complexity level of the models used) in the virtual environment. These volumes are adequate to create a medium scale emergency incident simulation, as was implemented in this research. The system can render with an acceptable performance as long as the graphical demands are kept below this limit. The FPS can be maintained at an effective level by either limiting the number of complex shapes or editing the models to remove detail and dropping vertices from curved surfaces.

\subsubsection{Roundtrip time and real-time data updating frequency}

Network performance is another important feature of CVE. Network performance can be reflected by roundtrip time, which is the length of time it takes for a signal to be sent plus the time for a response of that signal to be received. It can be captured by a built-in script in SIEVE-VTS. A gradual increasing of the real-time data update frequency was tested on different scenarios in the virtual environment. 


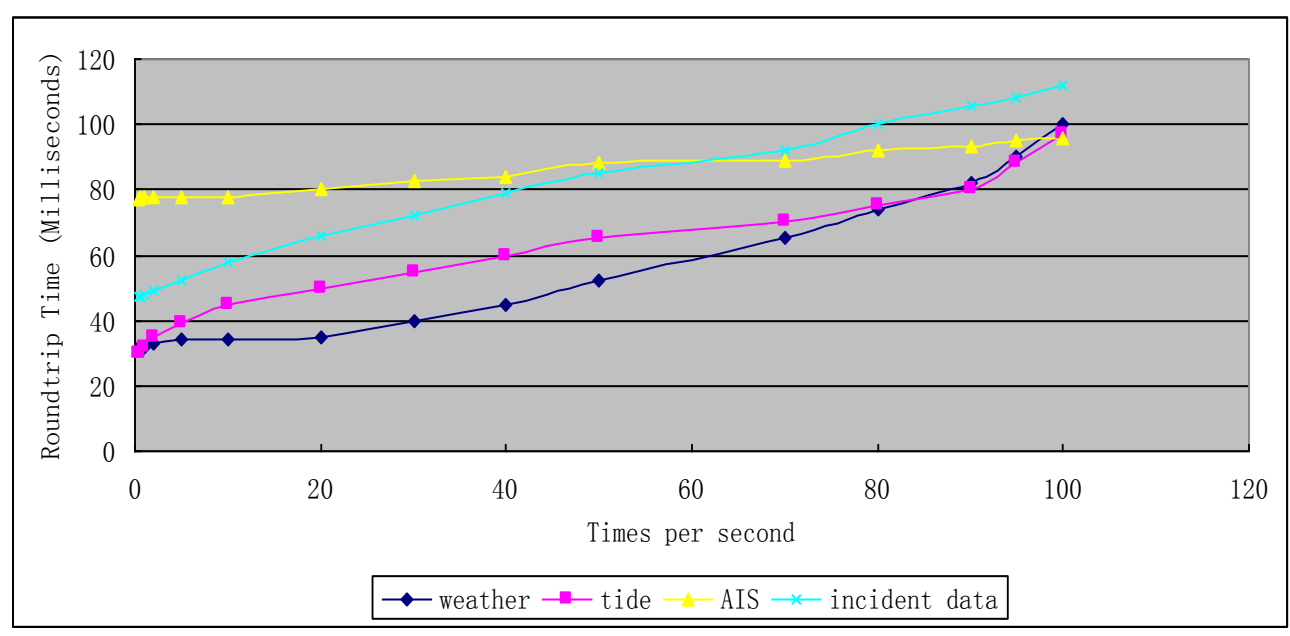

Figure 5.2: Roundtrip time and updating frequency

Figure 5.2 shows the results of the experiment. Positive relations are found between the variables of updating frequency and roundtrip time. When real-time data is updated frequently, the lag in different data is obvious. As seen from the graph, it can be concluded that:

1. There is an increase in roundtrip time when the updating frequency is raised for all types of data. This is because frequent updating requires additional time for memory loading of data and rendering, therefore the server needs a longer period to receive an acknowledgement packet.

2. The system can support more than 120 times per second updating frequency of real-time data, while the roundtrip time is lower than 150 milliseconds in most cases. The system can still deliver satisfactory network performance even if there is a large amount of data, for example, when hundreds of fire fighters are being tracked with updating GPS signals, or when multiple vessels are updating their location information. The update frequency of the data used in this research ranges from 6 seconds to more than 1 hour. The updated information can be easily rendered in the virtual environment. 


\subsection{Functional Test and Feedback}

SIEVE-VTS was demonstrated in May 2011 to a small user group to gain feedback on its functionality. The group was composed of two scientists, two technicians and one team leader, who were considered as the potential users of the system. The group were shown several scenarios, and asked to individually state preferences, answer questions and give general comments on the visualisations using questionnaires (Appendix 2). The results of this demonstration give insight into which aspects are of importance in supporting emergency management via CVE.

Firstly, the group were shown two methods of real-time data representation, and they were asked how well each method can deliver information under emergency management circumstances. Real-time data included marine traffic (Figure 5.3) and incident locations and status (Figure 5.4), as detailed in Chapter 3. For real-time marine traffic, a greater number of group members considered that the CVE (B) can provide more information. Comments included: "show more detail and variations of the vessels movement", "more real-looking", and "clearer view of the appearance of the vessel". For incident location and status, there was no obvious preference for 2D maps (A) or CVE (B), but both achieved good scores. The reasons for supporting 2D maps (A) were: "observe more incident information on different locations simultaneously", "knowing the exact location of the incidents", "good for identifying types of incidents", and "easy for novices to use and navigate". The reasons for supporting the CVE view (B) included: "3D visualisations help to quickly locate the site of event", "More intuitive display and gives context to information in geographic sense", "appears more realistic", and "lively landscape with terrain and buildings". 
A

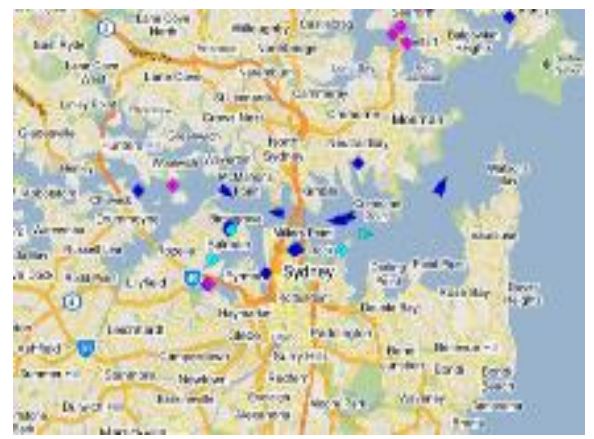

$\mathrm{B}$

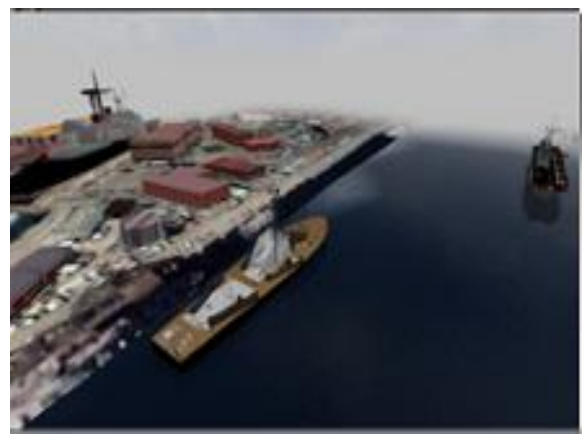

Figure 5.3: Comparison of marine traffic

A

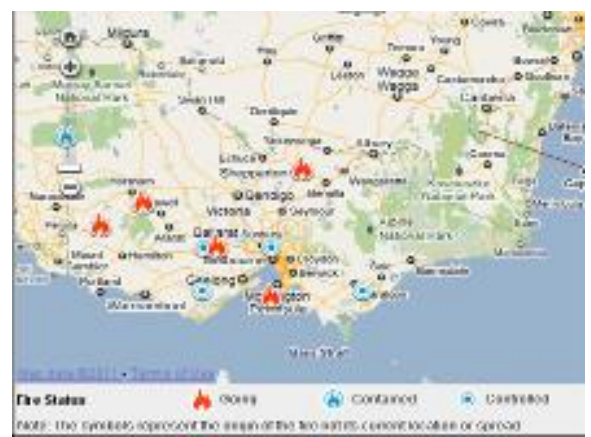

B

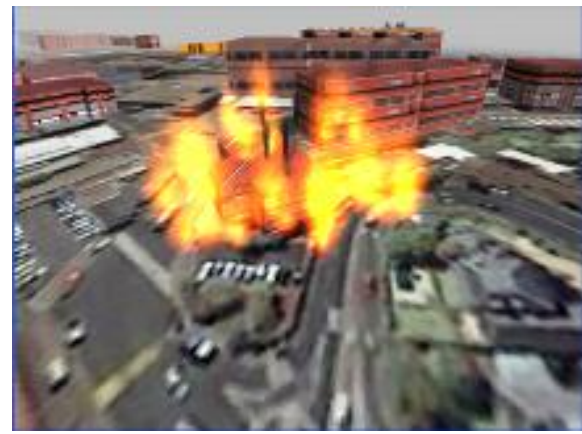

Figure 5.4: Comparison of incident location and status

The main difference between the two methods is that SIEVE-VTS renders real-time data in a 3D virtual environment, while the online maps use $2 \mathrm{D}$ icons to deliver the real-time information. Another difference is that online maps enable users to visualise information on multiple scales, ranging from local to global view, while SIEVE-VTS focuses to a limited extent, in this case a $2.5 \mathrm{~km} \times 2.5 \mathrm{~km}$ area. The preferences and comments on different representations indicate that the 3D environment is valuable to convey more information in emergency management, as it helps users quickly orientate themselves and locate the site of an event, and gives a more intuitive and realistic demonstration. In addition, based on the survey outcome, it can be found that the capabilities of enhancing the ease of use, supporting multiple scale visualisation, and integrating accurate spatial information are also significant when representing real-time data for emergency management.

The second question is about navigation functionality within SIEVE-VTS. Users were 
shown an incident scenario using historical data, and then asked to determine the direction and distance of the incident location from the current location of the avatar (Figure 5.5). In the first case, the group were shown a fire incident occurring over a hundred metres away from the avatar, and no ancillary information about the location and distance was provided. Most people found it difficult to make the estimation, and only those who were familiar with the area could speculate based on other reference objects, such as buildings and roads. In the second condition, the group were shown another fire incident with different direction and distance to the avatar, and additional information was provided, including the respective position of the avatar and incident in latitude and longitude. Most group members were able to determine that the fire incident was to the northwest of the avatar, approximately 250 to 300 metres away.

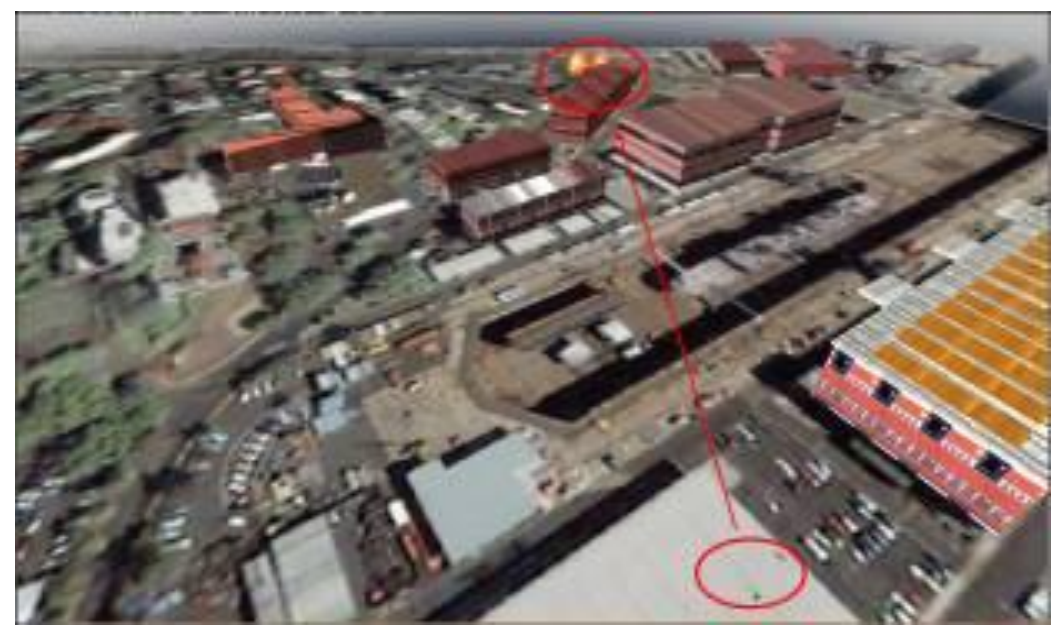

Figure 5.5: Positioning in SIEVE-VTS

In the first case, some group users could orientate themselves and navigate to the target location, because they could interpret the concealed spatial information in the 3D virtual environment, such as the position and scale of buildings, roads and trees. However, it is challenging for novice users to employ such skills. In the second case, there was an obvious improvement in the correctness of estimation when users were provided with spatial information. This suggested the importance of providing positioning or navigation tools in the virtual environment for emergency management purposes, for example, when a user clicks two buildings, the distance between them 
can be displayed. Quantitative measuring tools are useful to make accurate assessments of location, distances, and scales.

In the following question, users were introduced to three tools for emergency management, including Crowd Simulations for Military Operations (COSMOS) (Nanyang Technological University, 2010), a 3D real-time strategy based training platform for handling and managing crowds in military operation, UnrealTriage (McGrath \& Hill, 2004), a game-based simulation of mass casualty incidents for emergency response, and SIEVE-VTS (Figure 5.6). The group were asked to score and comment on the tools based on their potential utilisation within emergency management. On average, COSMOS was scored as 4.0. The comments included: "more detailed models for indoor environment", "shows more detail and variations in characters", "predicting and modelling crowd behaviours", and "might be useful for evacuation plans". UnrealTriage was scored as 2.9, and the reasons given for selecting it included: "realistic virtual environment" and "possible more human-computer interaction". SIEVE-VTS was scored at 4.3 with the following comments: "linking to underlying real-time data is useful", "great relevance to modelling certain scenarios", "allows quick identification of incident and event location", and "ideal for route planning and emergency exercise".

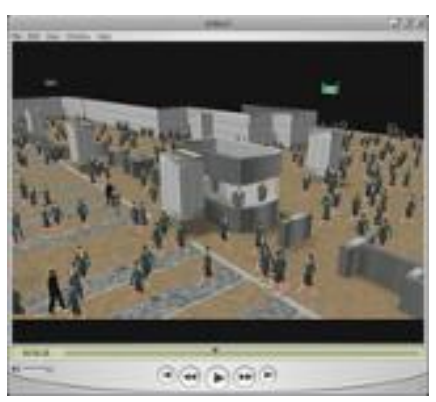

A

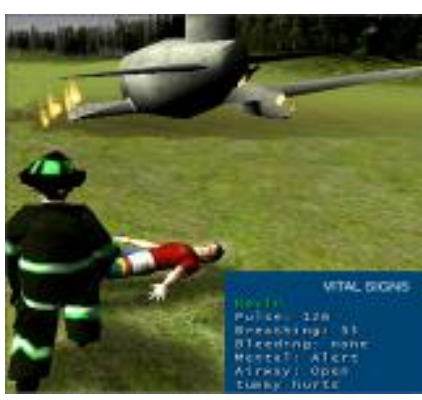

B

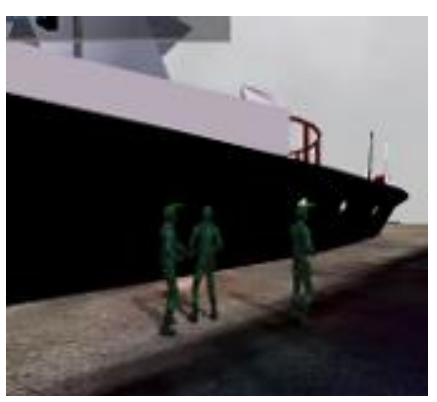

$\mathrm{C}$

Figure 5.6: Tools for emergency management: A) COSMOS; B) UnrealTriage; C) SIEVE-VTS

The three demonstrated tools share many common features: all of them are adapted from game engines, and they are all non-commercialised applications which do not 
provide comprehensive functionalities but aim to be used as auxiliary tools in emergency management. The scores of the tools do not show any overwhelming preference of users, especially given the small sample size. However, the comments on the tools indicate that elements of both of the static models displaying realistic detail and dynamic simulations using real-time data are very valuable for conveying information in emergency management.

In the next question, the users observed two emergency response scenarios using artificial data in SIEVE-VTS, and were asked to determine which is more suitable for emergency management (Figure 5.7). The first scenario (A) was performed by one avatar that communicated and interacted with AI characters responding to an explosion incident. The second scenario (B) was carried out by multiple users who collaborated with each other via a LAN connection. As a result, most group members selected B due to the following reasons: "users have more freedom of controlling", "the collaboration between human character and AI characters in A looks fake", and "more user interactivities in B".

A

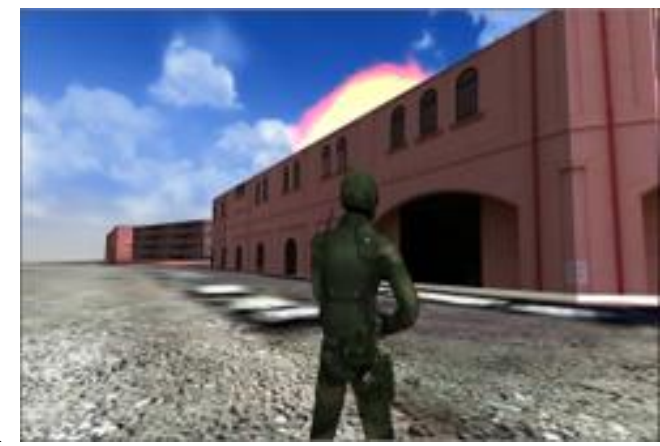

B

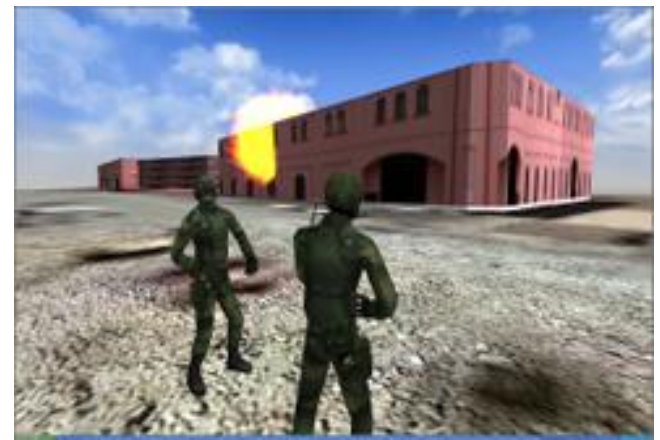

Figure 5.7: Comparison of single and multiple users

Overall the group preferred multiple users' collaboration using a LAN connection, rather than a single avatar in the virtual environment. On the one hand, this suggests that the AI performance still needs improving in SIEVE-VTS; on the other hand, it is worthwhile to generate multiplayer functionality in terms of emergency management using virtual environments. This function can assist collaboration between multiple 
users at different locations, improve information exchange and sharing, and facilitate leaders' guidance and instruction via the network. This function might be better tested if users were given the opportunities to interact directly with SIEVE-VTS.

Finally, users within the group were shown visualisation results displaying the simulation of real-time data, including weather, oceanographic, and marine traffic information. They were then asked to estimate the corresponding magnitude of elements in the simulation. In the first scenario (Figure 5.8), users were shown the study area in fine weather conditions and told the visibility was "8 km", after that, they were shown a severe weather condition with a relatively low level of visibility, and asked to estimate the visibility. In the second scenario (Figure 5.9), a low tide was shown to the group and the relevant water level $(0.12 \mathrm{~m})$ was also provided. Following this a high tide was demonstrated and users were asked to estimate the water level. In the third scenario (Figure 5.10), a cargo ship steered out of Sydney Harbour with low speed and users were asked to guess the speed of the ship. Users were able to observe, estimate or calculate based on the scenario and their own knowledge.
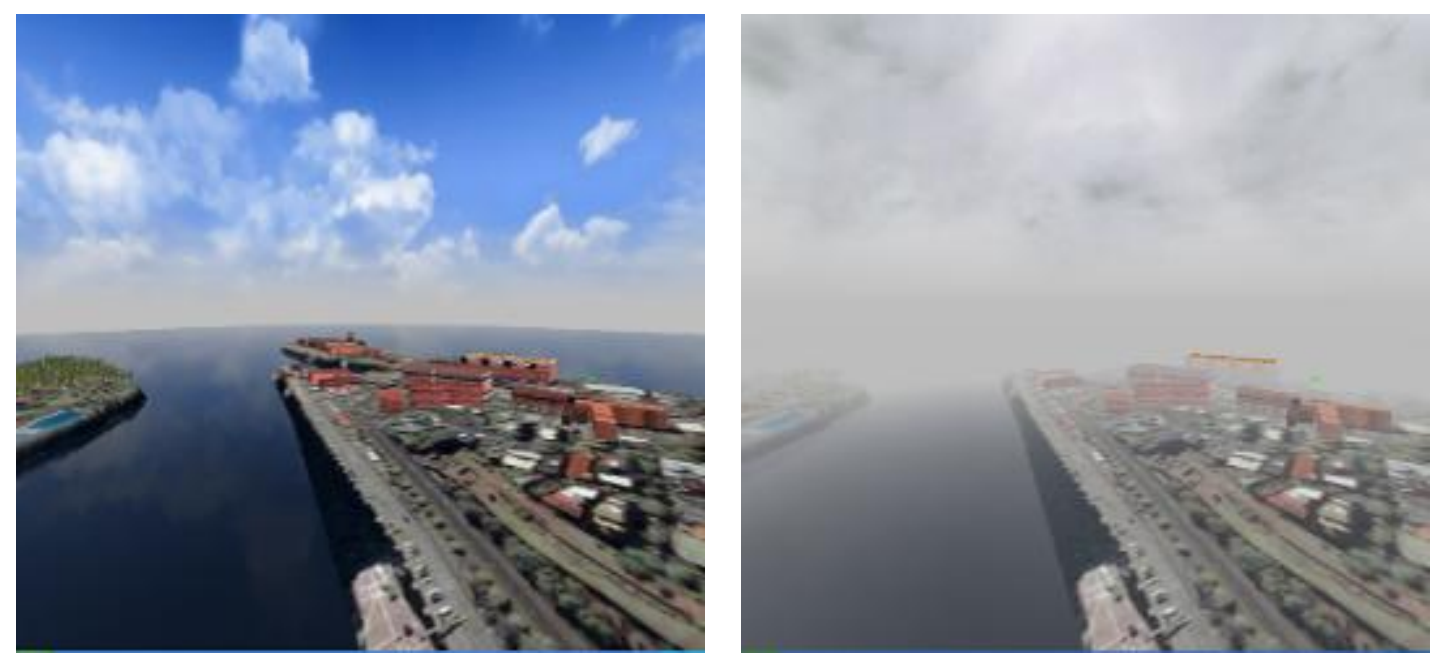

Figure 5.8: User's estimation of visibility 

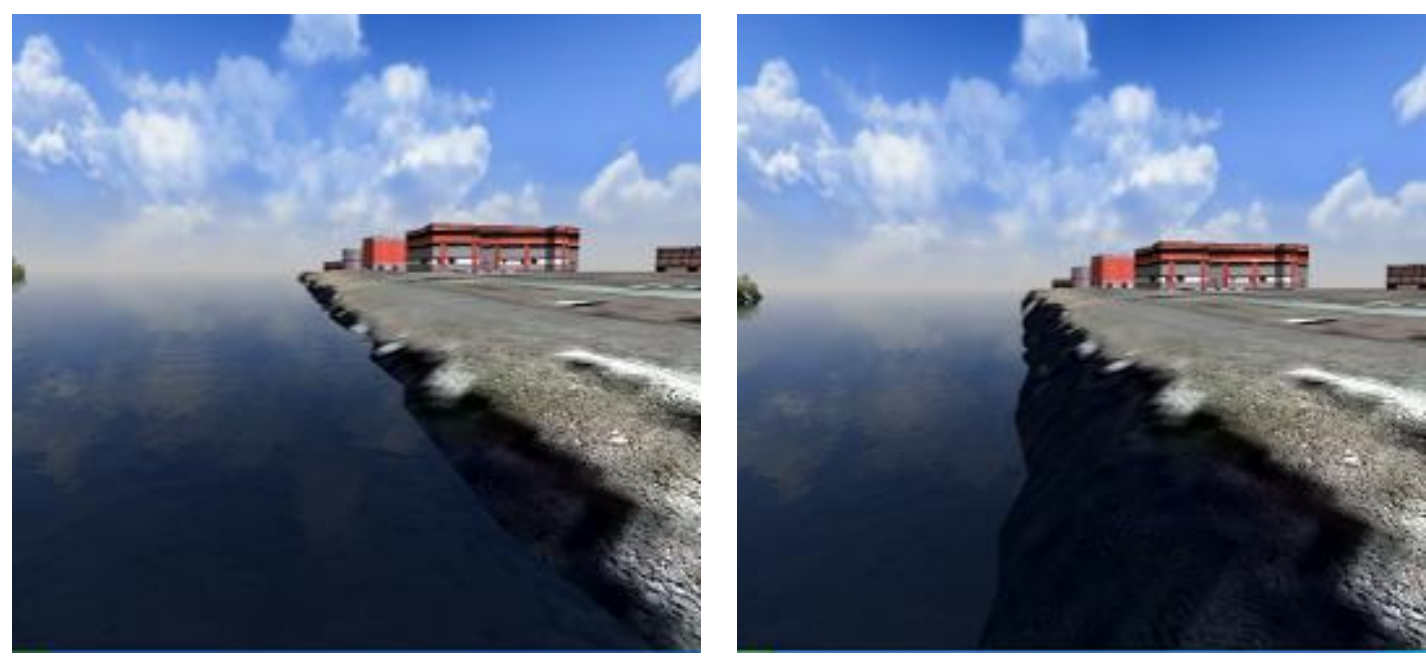

Figure 5.9: User's estimation of water level

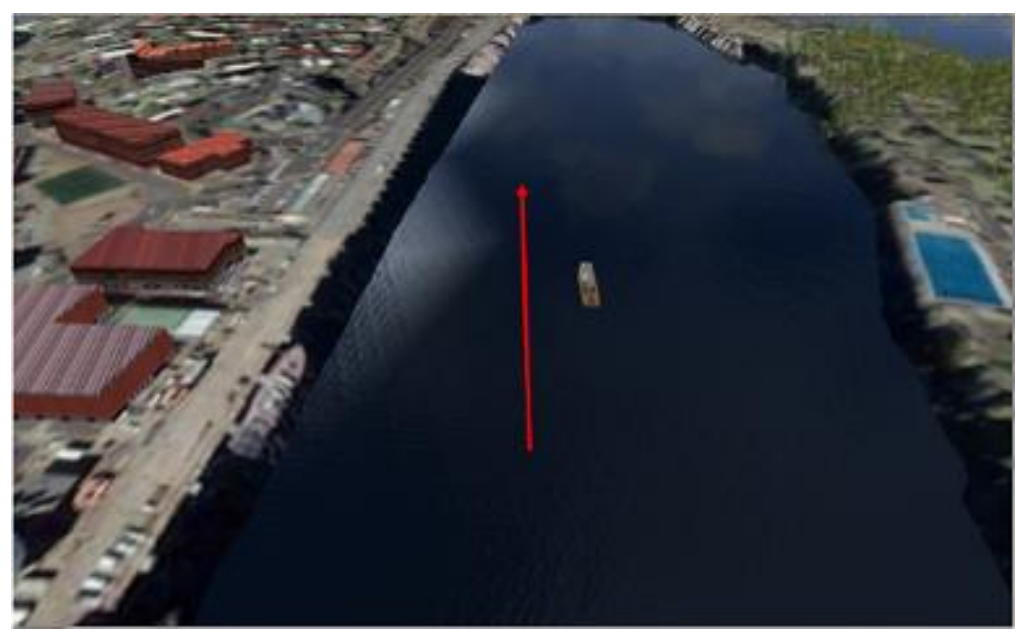

Figure 5.10: User's estimation of ship speed

In the first scenario, the estimation of the visibility ranged from $600 \mathrm{~m}$ to $1,500 \mathrm{~m}$, and the average value, of 1,080 m, was $19 \%$ higher than the true value. In the second scenario, the estimation of water level ranged from $1 \mathrm{~m}$ to $3 \mathrm{~m}$ with an average value of $2.1 \mathrm{~m}, 10 \%$ higher than the true value. In the last scenario, the average estimation of the ship speed was $15 \mathrm{~km}$ per hour, and ranged from $10 \mathrm{kph}$ to $30 \mathrm{kph}, 10 \%$ lower than the true value. The results indicate that SIEVE-VTS can generally provide users with a qualified simulation that is similar to the real world, while still needing enhancement on the accuracy of some elements. It is also necessary to implement a quantitative tool to assist users to identify the quantity of visualisation elements. 


\subsection{SIEVE-VTS Usage}

After the demonstration, there was a formal interview with the DSTO users. They were asked to share their views on the potential usage of SIEVE-VTS. The comments were also collected in the questionnaires and summarised within this section. Two usage scenarios were discussed to define the usability of SIEVE-VTS in its current form.

\subsubsection{Emergency monitoring and response using SIEVE-VTS}

SIEVE-VTS collects data from the real world and simultaneously visualises it in the virtual environment, therefore it can be utilised for emergency monitoring and response. Emergency managers and decision makers can observe real conditions of emergency incidents through the virtual environment, and take further steps when discovering incidents. They can also query and browse incident details and relevant environmental data through the user interface. From the interviews and comments, it is evident that the users believe that SIEVE-VTS can be utilised for emergency monitoring and response in the following steps:

Step 1. Monitoring specific areas:

By collecting multiple types of data, SIEVE-VTS is able to provide the basic conditions of a certain area. Such data include weather conditions, oceanographic data, local traffic, incident location and status. Weather and oceanographic data is useful for forecasting the future conditions and analysing the probability of hazards such as storm and tsunami. Traffic conditions can provide basic information of harbour environment security. The system also detects whether there are any incident reports updated from mobile devices. The information can be manipulated by an incident monitoring centre and distributed to the regional and local emergency offices through Web services. 
Step 2. Incident detection and early warning:

When an incident is detected at a certain location, detailed information can be displayed in the virtual environment. As this occurs, support staff can check the authenticity of the situation and the accuracy of the data by obtaining additional information from other organisations, and inform certain agencies such as fire brigade, first responders and ambulance services of the emerging conditions.

Step 3. Emergency response:

Effective and fast response to the warning is carried out in this step. The emergency manager will assign different tasks to agencies based on their locations and availability. When the first responders receive the warning message, they may, if safe, quickly access the accident scene equipped with wearable sensors and GPS devices. Such tools can help detect various environmental parameters, perform accurate positioning, and collect route and evacuation information. Meanwhile, dispatchers at the monitoring centre can track the responders and follow incident events using the latest update information. The dispatchers are able to see the locations of units and obtain a near real time view of incident locations and status through the virtual environment. Information can also be shared with other users, such as personnel from collaborating agencies, local officials, and decision makers, who can be fully briefed while being located in different places.

The setup of a monitoring and warning system to detect potential emergencies allows for better response and planning. The integrated system collects, analyses and interprets the monitored data, which can be utilised to make decisions early enough to minimise unnecessary concern and inconvenience to the public, and to save more lives and properties.

Further discussion with users in DSTO also suggested some future functionality for utilising SIEVE-VTS as an emergency monitoring tool: 
- Link to surveillance data such as video camera feeds.

- Integrate more types of real-time data.

- Design more user friendly interface (Fuhrmann \& Pike, 2005).

- Connect closely with other organisations such as police, fire and ambulance.

\subsubsection{Emergency training using SIEVE-VTS}

SIEVE-VTS is capable of simulating emergency scenarios in the virtual environment, thus it could also be used for emergency training. The opportunity for collaborative emergency training was also discussed and details provided by users from DSTO. It would involve 4 steps:

Step 1. Briefing and preparation:

Firstly, the training facilitator announces the outline and training content of the emergency scenario. Meanwhile, the trainees make preparations for the exercise, study the geographical information of the scenario with the assistance of facilitator, and familiarise themselves with training tools, such as the command and control in the virtual environment using computers.

Step 2. Role and task assignment:

Trainees are automatically divided into different roles by choosing specific characters in the GUI. Each one is allocated a specific role for executing collaborative tasks. Trainees with different background and skills can contribute their knowledge and experience according to their given tasks. When confronted with problems, they could use collaborative discussion within the virtual environment, evaluate opinions from all aspects, and produce a solution covering various conditions.

Step 3. Regular exercise: 
When the training starts, the facilitator can select a pre-designed emergency scenario, control the trainees' performance and give advice when there are significant errors. Trainees will respond to any emergency incident and act according to the circumstance including the real-time situation, for example, by extinguishing a fire, controlling the incident, or evacuating affected civilians. Trainees can also collaborate with each other on the Internet or LAN to analyse the scenario, for example, the position of hostile and friendly forces, and the status of the incidents. Meanwhile, auxiliary data such as weather, oceanographic data, and AIS can be collected and incorporated in real time, which can provide trainees with an environment in which they can respond to near real situations. Adding real-time data into a scenario based on conditions at the time of training would make each training scenario new and different, and more in line with what trainees know the real world to be. Simulations using real-time data were previously discussed in more detail in Section 4.3.4.

Step 4: Evaluation and summary:

When the virtual emergency training is finished, the instructor leads the trainees to evaluate the procedures and solutions. Usually, each scenario has one or more optimised solutions given by the instructor to guide the trainees and comment on proposed solutions from training groups at different stages of the scenario training. Questions, discussions, and debates on proposed and reference solutions are carried out. Trainees reflect on how command and control is operated, how to solve issues by cooperating within groups, and how to operate the procedure of a specifically designed scenario. The knowledge from this training process may be useful for developing new scenarios, enhancing the trainees' knowledge and correcting potential mistakes in a real response. In this step, the After Action Review (AAR) system can be applied for improving the training effect.

These are the general steps of virtual emergency training. In fact, scenarios cannot only be used for emergency training in Sydney Harbour, but can also be adopted into other fields such as fire fighting, medical rescue, and so forth. Since this research lays 
particular emphasis on technical aspects, more detailed training methods are not illustrated.

\subsection{Summary}

This chapter summarised the testing and evaluation of SIEVE-VTS to meet the performance and functionality requirements proposed in previous chapters.

In Section 5.2, performance evaluation regarding rendering and networking capability was designed and introduced. In relation to the objective of "simulating various types of incident scenarios with acceptable rendering time", the experiment demonstrated that:

- The rendering frame rate reduces with the increasing of scene complexity.

- $\quad$ SIEVE-VTS is capable of simulating large amounts of incident scenarios composed by hundreds of virtual objects, while maintaining an acceptable rendering frame rate.

When testing "sharing real-time spatial data via network", the experiment showed that:

- It is worthwhile making the effort to generate network functionality in terms of sharing real-time data for emergency management using virtual environments.

- There is an increase in networking load when the updating frequency raises for all types of data.

- $\quad$ SIEVE-VTS can properly accept and render the frequently updated real-time data in the virtual environment.

In Section 5.3, a demonstration was given to a small group of 5 people, the feedback and comments from which were instructive. The responses may be useful for 
formation of complete testing of similar visualisation tools in the future. The findings relevant to the functional requirements are listed below.

With regard to "improving the authenticity of CVE by integrating real-time data", it was found that:

- A combination of real-time data and CVE provides more information in emergency management than either alone.

- Using real-time data, SIEVE-VTS can generally provide users with a qualified simulation which is similar to the real world.

- Linking underlying real-time data is useful for emergency management, especially for emergency response.

When it came to "representing real world spatial information within the CVE", the feedback was that:

- Spatial information can be accurately displayed in SIEVE-VTS, therefore the users can orient themselves and other objects in the virtual environment.

- It is important to provide positioning or navigation tools in the virtual environment for emergency management purposes.

- Auxiliary tools, such as positioning or navigation gadgets should be implemented in order to assist users to identify the spatial information in the virtual environment.

In terms of "offering comprehensive simulation, training and monitoring as an emergency management tool", the user group agreed that:

- $\quad$ SIEVE-VTS shows great detail in the virtual environment and is able to simulate various types of incident scenarios. 
- It is useful to link real-time data to allow for quick identification of incident and event location, which is ideal for route planning and emergency response.

With reference to the functional requirement of "extending mashup technology from 2D to 3D environment", it was found that:

- The 3D environment is valuable for conveying more information in emergency management, and helping users quickly orientate themselves and locate the site of an event.

- It has a more intuitive display and gives context to information in geographic sense.

- It appears more realistic by integrating lively landscapes with terrain and buildings.

In addition, the usage of SIEVE-VTS was illustrated in Section 5.4. Detailed applications in emergency training and monitoring are evident to potential users. 



\section{Chapter 6 Conclusions and Further Outlook}

\subsection{Introduction}

This thesis presented the design and development of a novel prototype software that facilitates emergency management via a Collaborative Virtual Environment using real-time spatial information. The system, Spatial Information Exploration and Visualisation Environment - Virtual Training System or SIEVE-VTS, was developed based on a game engine. It automatically integrates real-time data from multiple online sources, models and simulates emergency incident scenarios using such data. The advantage of the system is that it facilitates collaboration and communication among users for emergency response or training. It also benefits the processes and outcomes of emergency management by enhancing engagement and decision making of first responders, emergency managers and other stakeholders.

The thesis started with the technology and application background of emergency management. The major problems in emergency management using virtual environment tools were exposed. Insufficiency of authenticity in simulation, low timeliness in data delivery, and unsatisfying usability of emergency management tools have been highlighted as the main issues. Subsequent discussions laid the foundation, clarified the problem and set the objectives for this research.

Chapter 2 reviewed relevant tools and technologies for emergency management. It included discussion of advanced tools for emergency monitoring and training, 3D spatial information visualisation system, as well as real-time data integration and modelling technology. The virtues and restrictions of these techniques were evaluated to specify the requirements for the design of a new emergency management system for decision support.

In Chapter 3, a prototype system SIEVE-VTS was proposed to solve the issues 
mentioned in previous chapters. The overall design and the modules comprising the system were described. Functionalities including integrating, translating and visualising marine-related real-time information were illustrated in detail. The connection of real-time data and visual simulations in CVE can enhance the capabilities of emergency management.

A case study of emergency incident simulation in the Sydney Harbour area was carried out and described in Chapter 4. It established that using SIEVE-VTS, especially the real-time data collection and visualisation components, can significantly improve the effect of emergency simulation compared with traditional technology.

SIEVE-VTS was demonstrated to project collaborators. Chapter 5 discussed the feedback from them, confirmed the effectiveness and investigated potential applications of SIEVE-VTS.

As the final part, this chapter revisits the research objectives, outlines the contribution made by this research, summarises the findings and suggests future directions to be taken in a broader context.

\subsection{Research Achievement}

SIEVE-VTS provides the capability of simulating dynamic scenarios in the virtual environment. As was illustrated in the previous chapter, SIEVE-VTS was developed from SIEVE, which provided a 3D visualisation environment with GIS connections. SIEVE manages static objects in the virtual environment, including terrain, buildings and vegetation. It also allows for simulation of long term variations like growing forests, and short time variations like vegetation swaying in the wind. Such simulation is useful but inadequate for simulating time-pressed emergency incidents. SIEVE-VTS takes a further step by using an object-oriented method, assigning each object attributes containing variables that describe spatial and temporal status. Therefore, 
dynamic variations can be simulated in the virtual environment, such as weather, oceanographic information and traffic conditions, which meets the overall objective of this research. The research objectives in Section 1.3 are revisited and discussed here:

- Investigate and design new techniques to integrate real-time data from multiple sources in a cost-efficient way.

- Model and visually simulate real-time data (such as weather, traffic condition, incident location) in an emergency management situation.

- Develop a virtual simulation platform and an updated user interface for emergency management in CVE.

- Test and evaluate the functions, performance and usability of the developed system through a client driven case study.

To investigate and design new techniques to integrate real-time data from multiple sources, SIEVE-VTS extends the mashup technique from 2D maps to the 3D virtual environment. Mashup, as discussed in Chapter 2, is a web application that integrates data from different sources into one single tool. Currently, most applications are implemented in the 2D environment, such as online maps. SIEVE-VTS contains an interface for collecting real-time data, which makes full use of mashup and adapts it into the $3 \mathrm{D}$ virtual environment. The architecture is similar to the traditional technology but explores new features and functionalities. Applying this technology to a 3D environment can provide users with a more intuitive and realistic display and give more geographic context to the information.

To model and visually simulate real-time data in an emergency management situation, a full-featured 3D game engine was utilised and integrated into this system. As was discussed in Chapter 2, integrating real-time data into the system enables users to explore on-site incidents or training scenarios in a high authenticity environment, and 
to monitor emergency situations more effectively. To do this, a method was proposed to collect data from online content providers and other agencies, transfer it to a centralised server, and visualise the results in CVE. This methodology improves the fidelity of emergency simulation and enhances the efficiency of automatically creating virtual worlds. More specifically, RTDTranslator was designed for translating from the original parameters to visual results which can be supported by SIEVE-VTS. RTDSimulator, the visual simulation component, was also developed for users to visualise the final results. Different simulation methods and algorithms were applied on four types of data: weather, oceanographic information, AIS, and incident location and status.

To develop a virtual simulation platform and an updated user interface for emergency management in CVE, SIEVE-VTS has provided a direct interface for users to collect and visualise real-time information. It reduces the complexity and time costs for developing interactive visualisation environments. SIEVE-VTS integrates functionalities including data manipulation, physics simulation, lighting and shadowing, AI, networking, scenario management, and terrain rendering. Although it is a highly integrated system, it is still flexible for further development such as adding third party libraries. For example, TinyXML library was integrated in SIEVE-VTS for parsing XML files. SIEVE-VTS is designed as a client-server architecture in order to enhance the collaboration and communication between the users. For emergency monitoring, several agencies can exchange ideas, share data with each other to facilitate decision making processes. For emergency training, the trainees can be immersed into one common virtual environment and collaborate with each other, regardless of their physical location.

To test and evaluate the functions, performance and usability of the developed system, the rendering effect and network performance of SIEVE-VTS was tested and found adequate for quite complex environments. In addition, SIEVE-VTS was demonstrated to a small user group from DSTO, Sydney, in order to evaluate its functionality, 
including 3D data mashup, spatial information support, dynamic simulation, and multi-user collaboration. The users also shared their views on the usability of SIEVE-VTS in a formal interview. This evaluation can be more comprehensive, for example, utilizing formal usability studies with larger number of students to get a large pool of data on general system usability and then with a smaller group of experts for utility of the system in real life use.

\subsection{Further Research}

\subsubsection{Collecting high quality real-time data}

SIEVE-VTS has the capability of collecting, translating and visualising real-time data. The quality of that data is a key factor worth serious consideration. Data quality is the state of completeness, validity, consistency, timeliness and accuracy that makes data appropriate for a specific use (Government of British Columbia, 2010). As explained in Chapter 3, currently there are some limitations on data quality in SIEVE-VTS due to the data source and redistribution method. SIEVE-VTS can be better utilised if data quality can be improved through future work.

Among the elements of data quality, accuracy measures the degree to which data can correctly reflect the real world object or an event described (English, 2003). The accuracy of real-time data in SIEVE-VTS mainly relies on the on-line data source, and is also related to the losses in the redistribution and reconfiguration process. In practice, it is not easy to improve the data accuracy from a single data source. However, it is possible to find high quality data sources in both public and private sectors. In the future, it would be ideal to design a new interface for collecting data from more reliable sources, and utilise data that complies with international standards. For improving data accuracy in the transferring processes, automatic comparing and optimising algorithms can be realised to distinguish the most accurate data by comparison with the historical data. 
Data timeliness determines whether data can be delivered to end users while the data is still current and as it is needed. In the context of emergency management, it is valuable to stream real-time data directly into a central database with minimum delays. One solution to improve data timeliness in SIEVE-VTS is incorporation of Sensor Web Enablement (SWE). The SWE standards proposed by Open Geospatial Consortium $(O G C)$ enable developers to make all types of sensors, transducers and sensor data repositories discoverable and accessible via the Web (Open Geospatial Consortium, 2010). Spatially distributed autonomous sensors can cooperatively monitor and store real-time data including: physical or environmental conditions, such as temperature, atmospheric pressure and pollutants; natural and man-made disasters, such as tornado, flood or earthquake; and traffic conditions on land and ocean (Figure 6.1). Integrating SWE to SIEVE-VTS may include: digital cameras distributed to capture the overall situations; traffic sensors to record traffic conditions, track and locate vehicles; mobile weather stations to gather information about the local weather conditions, such as wind, precipitation or humidity, and monitor extreme weather hazards. The captured information can be distributed over the Web immediately after collection, and directly sent to SIEVE-VTS and visualised for emergency responses. Under some circumstances it may be necessary to consider issues associated with security and privacy in relation to the provision and visualisation of real-time data.

Using SWE has the following advantages: 1. users can retrieve data directly without any delay caused by other content providers in the intermediate process; 2 . the quality can be improved since there is no intervening process which may cause data losses; 3.SWE has been proposed as a standard under $O G C$ and has already been implemented in various fields for emergency management (Jirka, Bröring, \& Stasch, 2009; Babitski et al., 2009; Chou, Fang, \& Chung, 2010; Markovic, Stanimirovic, \& Stoimenov, 2009). The integration of SWE with SIEVE-VTS can also facilitate data exchange and interoperability. 


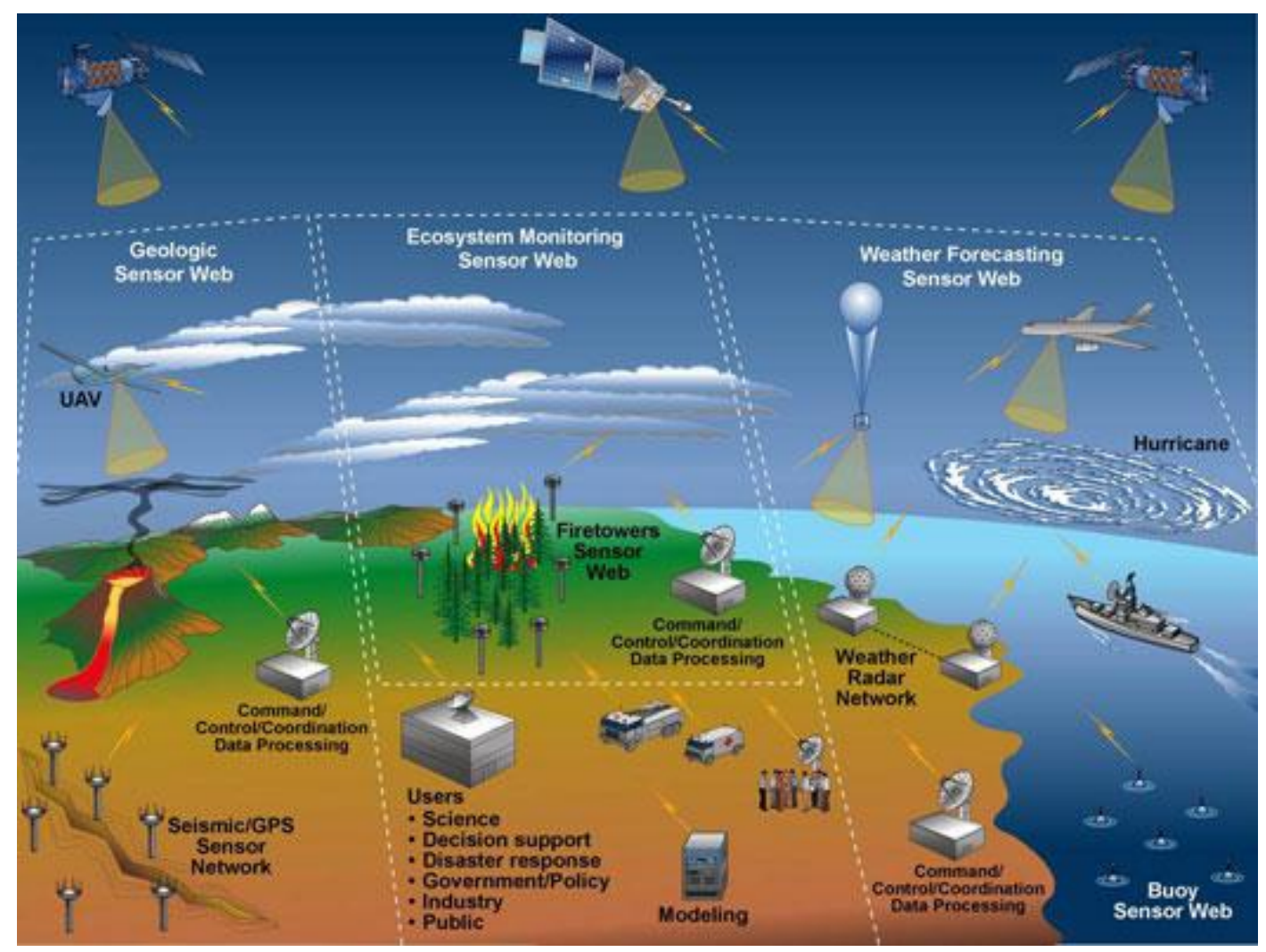

Figure 6.1: Sensor Web Enablement (Franklin, 2007)

Data completeness is another aspect of data quality, it is the extent to which the expected attributes of data are provided. Additional attributes can be incorporated to form a solid dataset based on the emergency situation for SIEVE-VTS. For example, in emergency response within an urban area, traffic information can be collected and displayed in the monitoring centre, to assist the emergency responders to acquire the latest traffic situation and make appropriate route planning. Auxiliary information about an emergency affected area can be collected as well, including data on injury and casualty, environmental damage, and property damage. A more complete dataset can provide emergency managers and first responders with critical information during a major emergency or crisis.

\subsubsection{Improvement on scenario simulation}

SIEVE-VTS is designed as an emergency management platform with basic functions of virtual simulation, networking with client-server architecture and game AI. 
However, there are further enhancements that could make it a more practical platform with additional advanced features.

One potential feature involves the 3D object models that are used to build landscape and scenarios in the virtual environment. Currently SIEVE-VTS provides limited types of models including buildings, vegetation, vessels and human characters. A full 3D object library with multiple file formats is a necessary complement (Bishop et al., 2009). New objects would include, but are not limited to: characters of different groups with pre-designed gestures and actions; diversified kinds of military and commercial ships; emergency response teams such as ambulance, police force and fire brigade; and a full range of tools and equipment, such as fire extinguishers and explosion protection facilities. Such a library would facilitate automatic creation of virtual worlds, and enable the simulation of more types of events and incident scenarios.

Another potential feature is the dynamic scenario collaboration. The design of SIEVE-VTS is based on a game engine, which can use AI for simulating emergency incidents and human-computer interaction. Higher levels of AI can be implemented in the system for training and monitoring purposes. For example, path finding strategies can be enhanced to fully support the users' route planning and target searching; decision making processes can be strengthened using finite state machines or fuzzy logic; and team collaboration will be improved by strategic and tactical reasoning, and group behaviour simulation. Furthermore, the practical emergency management platform can be enhanced if a new user-interface is designed for administrators and trainees. Administrators may be assigned with the ownership rights of SIEVE-VTS, such as editing, adding or deleting objects into the system. Trainees may have the rights to choose specific tasks, perform exercises, and engage in emergency management events. 


\subsubsection{Distributed interactive simulation}

When simulating the emergency incidents, either in training or response, there may be different systems used by participants in diverse locations, each of which have their own specific functions and advantages, and SIEVE-VTS may be one of these simulation platforms. Under this circumstance, the collaboration and coordination of different platforms is important. High Level Architecture (HLA) is a general architecture for distributed computer simulation systems. Computer simulations can communicate with each other using HLA, in spite of different computing platforms. Communication between simulations is coordinated by a Run-Time Infrastructure (RTI). Using HLA can help to synchronize the events designed on multiple platforms more efficiently, which guarantees that users see events and perform their interactions with minimum delays (Wang, Turner, Low, \& Gan, 2004; Santoro \& Quaglia, 2006).

One use case of HLA is to connect the training platforms used by DSTO, including a battle training system VBS2, marine traffic inspection platform, Joint Semi-Automated Forces (JSAF) and SIEVE-VTS. HLA can connect these platforms together for the use by trainees in different divisions.

Another use case of HLA is the creation of distributed simulations using SIEVE-VTS and Google Earth. Google Earth has the advantage of collecting online real-time data and offering various display and analysis, while SIEVE-VTS is capable of high fidelity simulation. Adapting HLA for the connection between Google Earth and SIEVE-VTS would allow virtual, live or constructive simulations on both systems, and improve the data quality of SIEVE-VTS (Figure 6.2). 


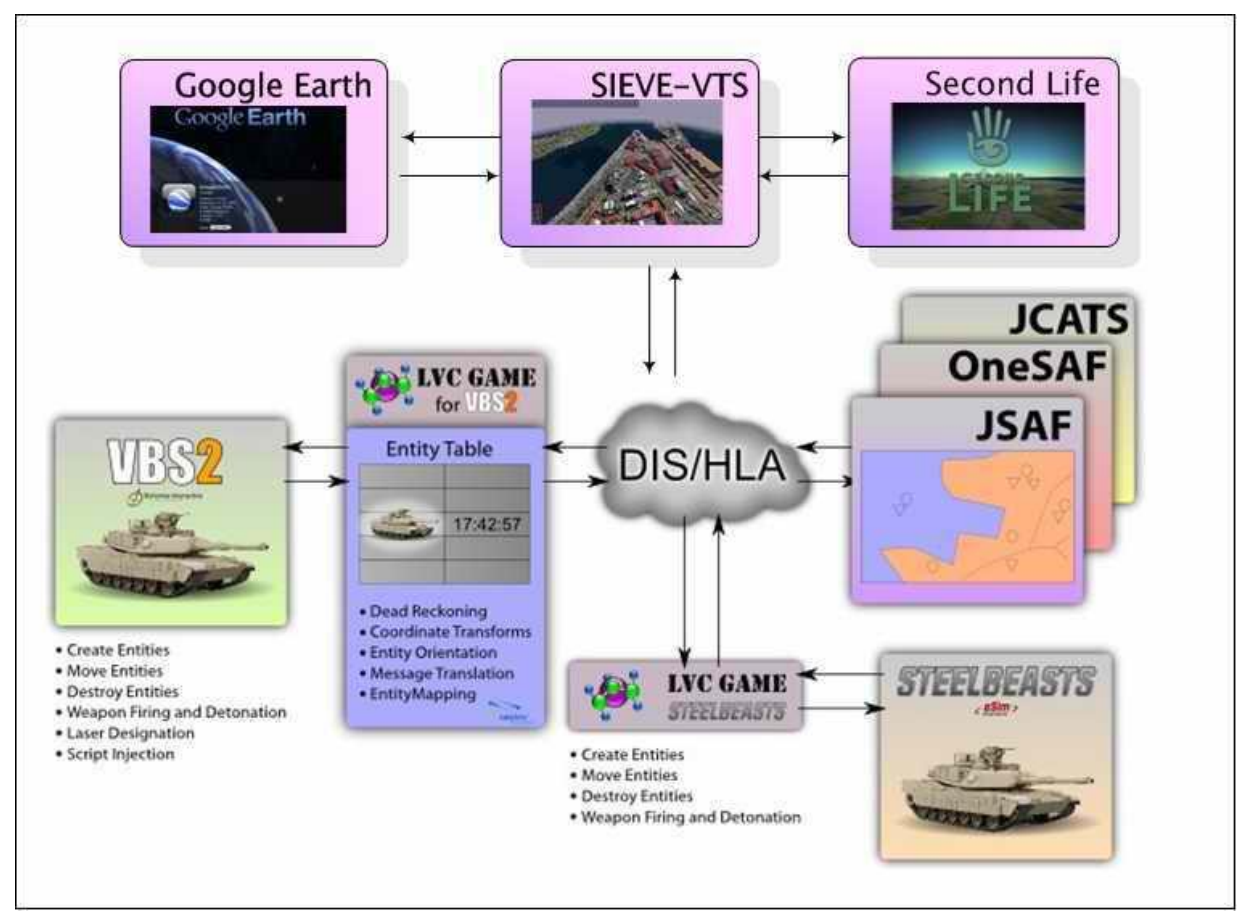

Figure 6.2: HLA for SIEVE-VTS (adapted from Calytrix Technology, 2010)

\subsection{Conclusions}

This research has closely connected real-time data and a Collaborative Virtual Environment. It was made possible both by developments in real-time data collection and modelling, and developments in 3D visualisation technology for spatial information. The aim of this research was to advance techniques for simulating real world events using CVE in the context of emergency management, and to facilitate better decision making processes. Based on this, an emergency management system, SIEVE-VTS was developed, which automatically collects real-time information from multiple data sources, and visualises it simultaneously in a game engine based virtual environment. In addition to this, SIEVE-VTS was used to simulate incident scenarios which form the basis of emergency training and monitoring. To provide a comprehensive emergency management and decision support system, user interactivities, game AI and network collaboration were adapted to enhance the capabilities of emergency monitoring and training. By using real-time data in CVE, 
SIEVE-VTS has successfully demonstrated its capability to facilitate emergency management with strong endorsement by potential users. 



\section{References}

3D Nature, LLC. (2010). Visual Nature Studio. Retrieved from http://3dnature.com/vnsinfo.html

Aigner, W., Miksch, S., Müller, W., Schumann, H., \& Tominski, C. (2007). Visualizing time-oriented data - a systematic view. Computers \& Graphics, 31(3), 401-409.

Alkems. (2010). Alkems. Retrieved from http://local.alkemis.com/local/index.htm

Alparslan, E., Ince, F., Erkan, B., Aydöner, C., Özen, H., Dönertaş, S., ...Özkan, M. (2008). A GIS model for settlement suitability regarding disaster mitigation, a case study in Bolu Turkey. Engineering Geology, 96(3-4), 126-140.

Apple Pty Ltd. (2010). iPhone4. Retrieved from http://www.apple.com/au/iphone/

Aquaveo, LLC. (2010). SMS: Natural Neighbor Interpolation. Retrieved from http://www.xmswiki.com/xms/SMS:Natural_Neighbor_Interpolation

Armstrong, M. P. (1988, November). Temporality in spatial databases. Paper presented at GIS/LIS'88 Conference, San Antonio, TX.

Astra Paging Ltd. (2010). AIS Hub. Retrieved from http://www.aishub.net/

Aurenhammer, F. (1991). Voronoi diagrams - A survey of a fundamental geometric data structure. ACM Computing Surveys, 23(3), 345-405.

Autodesk. (2010). Autodesk 3ds Max. Retrieved from http://usa.autodesk.com/3ds-max/

Babitski, G., Bergweiler, S., Hoffmann, J., Schoen, D., Stasch, C., \& Walkowski, A. C. (2009, December). Ontology-based integration of Sensor Web services in disaster management. Paper presented at $3^{\text {rd }}$ International Conference on GeoSpatial Semantics, Mexico City, Mexico.

Baldus, G., \& Kim, J. (2005, July). Integrating GIS and real-time vehicle tracking for county emergency response. Paper presented at 2005 ESRI International User Conference, San Diego, California.

Bayraktar, H., \& Turalioglu, F. S. (2005). A Kriging-based approach for locating a sampling site - in the assessment of air quality. Stochastic Environmental Research and Risk Assessment, 19(4), 301-305. 
Beepa Pty Ltd. (2010). FRAPS. Retrieved from http://www.fraps.com/

Beller, A., Giblin, T., Le, K. V., Litz, S., Kittel, T. \& Schimel, D. (1991, October). A temporal GIS prototype for global change research. Paper presented on GIS/LIS'91 Conference, Atlanta, GA.

Beni, L. H., Mostafavi, M. A., \& Pouliot, J. (2007). 3D dynamic simulation within GIS in support of disaster management. In J. Li, S. Zlatanova \& A. Fabbri (Eds.), Geomatics Solutions for Disaster Management (pp. 165-184). New York, NY: Springer-Verlag.

Bishop, I., Chan, P., Chan, T., Lau, A., Pettit, C., Stock, C., \& Syed, D. (2009, September). Object libraries: the next step in Spatial Data Infrastructure. Paper presented at the Surveying \& Spatial Sciences Institute Biennial International Conference, Adelaide, Australia.

Bista, S., \& Pack, M. L. (2008, May). Real-time massive data simulation visualization. Paper presented at 2008 IEEE Conference on Technologies for Homeland Security, Waltham, MA.

Black, R. (2011, April 12). Fukushima: as bad as Chernobyl? BBC News. Retrieved from http://www.bbc.co.uk/news/science-environment-13048916

Blasband, C., Bleak, J., \& Schultz, G. (2004). High fidelity, physics-based sensor simulation for military and civil applications. Sensor Review, 24(2), 151-155.

Bodum, L., Kjems, E., Kolar, J., Ilsøe, P., \& Overby, J. (2005). GRIFINOR: Integrated object-oriented solution for navigating real-time $3 \mathrm{D}$ virtual environments. In P. van Oosterom, S. Zlatanova \& E. M. Fendel (Eds.), Geo-information for Disaster Management (pp. 937-949). Berlin: Springer-Verlag.

Bohemia Interactive Australia Pty Ltd. (2010). White paper: VBS2. Retrieved from http://distribution.vbs2.com/docs/VBS2_Whitepaper.pdf

Bohemia Interactive Simulations. (2010). VBS2. Retrieved from http://www.bisimulations.com/

BOM. (2010a). Climate statistics for Australian locations: summary statistics for Sydney. Retrieved from http://www.bom.gov.au/climate/averages/tables/cw_066062.shtml

BOM. (2010b). Latest weather observations for the Sydney area. Retrieved from 
http://www.bom.gov.au/nsw/observations/sydney.shtml

Bonn, F., \& Dixon, R. (2005). Monitoring flood extent and forecasting excess runoff risk with RADARSAT-1 data. Natural Hazards, 35(3), 377-393.

Boon, J. D. (2004). Secrets of the tide: tide and tidal current analysis and predictions, storm surges and sea level trends. Philadelphia: Woodhead Publishing.

Botts, M., Percivall, G., Reed, C., \& Davidson, J. (2008). OGC® Sensor Web Enablement: overview and high level architecture. In S. Nittel, A. Labrinidis \& A. Stefanidis (Eds.), GeoSensor Networks (Lecture Notes in Computer Science, Volume 4540) (pp. 175-190). Berlin: Springer-Verlag.

Boulos, M. N., \& Burden, D. (2007). Web GIS in practice V: 3-D interactive and real-time mapping in Second Life. International Journal of Health Geographics, 6: 51.

Branco, P., Escalada, C., \& Santos, R. (2005). Real time 3D environment simulation applied to the disaster management field: our experience. In P. van Oosterom, S. Zlatanova \& E. M. Fendel (Eds.), Geo-information for Disaster Management (pp. 425-441). Berlin: Springer-Verlag.

Bursa, M. (1962). The theory for the determination of the non-parallelism of the minor axis of the reference ellipsoid and the inertial polar axis of the Earth, and of the planes of the initial astronomic and geodetic meridians from observations of artificial Earth satellites. Studia Geophysica et Geodetica, 6, 209-214.

Butler, D. (2006). Virtual globes: the web-wide world. Nature, 439(7078), 776-778.

Calytrix Technology. (2010). Calytrix bundles with VBS2. Retrieved from http://www.calytrix.com/news/newsitem/135/

Carrara, A., Guzzetti, F., Cardinali, M., \& Reichenbach, P. (1999). Use of GIS technology in the prediction and monitoring of landslide hazard. Natural Hazards, 20 (2), 117-135.

Castrillón, M., Jorge, P. A., López, I. J., Macías, A., Martín, D., Nebot, R. J., ...Trujillo, A. (2011). Forecasting and visualization of wildfires in a 3D Geographical Information System. Computers \& Geosciences, 37(3), 390-396.

Chadwick, R. (2010). Citizen weather observer program. Retrieved from http://wxqa.com/

Chen, F., \& Kalogeraki, V. (2005, May). Adaptive real-time update dissemination in 
distributed virtual simulation environments. Paper presented at the eighth IEEE International Symposium on Object-Oriented Real-Time Distributed Computing, 2005, Seattle, USA.

Chen, Y. F., Rebolledo-Mendez, G., Liarokapis, F., De Freitas, S., \& Parker, E. (2008, November). The use of virtual world platforms for supporting an emergency response training exercise. Paper presented at the $13^{\text {th }}$ International Conference on Computer Games: AI, Animation, Mobile, Interactive Multimedia, Educational \& Serious Games, Wolverhampton, UK.

Chen, R., Sharman, R., Chakravarti, N., Rao, H. R., \& Upadhyaya, S. (2008). Emergency response information system interoperability: development of chemical incident response data model. Journal of the Association for Information System, 9(3), 200-230.

Chen, A. T., Stock, C., Bishop, I. D., \& O'Connor, A. (2006, October). Prototyping an in-field collaborative environment for landscape decision support by linking GIS with a game engine. Paper presented at Geoinformatics 2006: GNSS and Integrated Geospatial Applications, Wuhan, China.

Chen, T., Stock, C., Bishop, I. D., \& Pettit, C. (2008). Automated generation of enhanced virtual environment for collaborative decision making via a live link to GIS. In C. Pettit, W. Cartwright, I. Bishop, K. Lowell, D. Puller \& D. Duncan (Eds.), Landscape Analysis and Visualisation, Spatial Models for Natural Resource Management and Planning (pp. 571-590). Berlin: Springer-Verlag.

Chicago Police Department. (2010). CLEARMAP. Retrieved from http://gis.chicagopolice.org/

Choi, J., \& Lee, J. (2008). 3D Geo-network for agent-based building evacuation simulation. In J. Lee \& S. Zlatanova (Eds.), 3D Geo-Information Sciences (Lecture Notes in Geoinformation and Cartography) (pp. 283-299). Berlin: Springer-Verlag.

Chou, T. Y., Fang, Y. M., \& Chung, L. K. (2010, October). Sensor Web Enablement for debris flow monitoring system in Taiwan. Paper presented at $22^{\text {nd }}$ International CODATA Conference, Cape Town, South Africa.

Churchill, E. F., Snowdon, D. N., \& Munro, A. J. (2001). Collaborative virtual environments: digital places and spaces for interaction. New York, USA: Springer.

Cole, J. W., Sabel, C. E., Blumenthal, E., Finnis, K., Dantas, A., Barnard, S., \& 
Johnston, D. M. (2005). GIS-based emergency and evacuation planning for volcanic hazards in New Zealand. Bulletin of the New Zealand Society for Earthquake Engineering, 38(2), 149-164.

Country Fire Authority. (2010). Incident Summary. Retrieved from http://www.cfa.vic.gov.au/incidents/incident_summary.htm

Davison, A. J. (2003, October). Real-time simultaneous localisation and mapping with a single camera. Paper presented at $9^{\text {th }}$ International Conference on Computer Vision, Nice, France.

Delaware Department of Transportation. (2006). All hazards evacuation annex, Transportation incident and event management plan, Sussex County, Delaware. Retrieved from http://www.deldot.gov/information/projects/tmt/pdfs/hurricane_evacuation/1_\% 20sussex_county_all_hazards_evacuation_annex_rev053107.pdf

De Troyer, O., Kleinermann, F., Mansouri, H., Pellens, B., Bille, W., \& Fomenko, V. (2007). Developing semantic VR-shops for e-commerce. Virtual Reality, 11(2-3), 89-106.

DIY Wood Boat. (2010). Rule of Twelfths and the Rule of Tenths for quick tidal calculation. Retrieved from http://www.diy-wood-boat.com/Rule_of_twelfths.html

Dutka, N. (2010). DTS exporter-3ds max 2010-100 details. Retrieved from http://www.garagegames.com/community/blogs/view/18261

Dyke, G., Gill, S., Davies, R., Betorz, F., Andalsvik, Y., Cackler, J., ... Verstappen, N. (2011). Dream project: Applications of earth observation to disaster risk management. Acta Astronautica, 68(1-2), 301-315.

Egbert, G. D., \& Erofeeva, S. Y. (2002). Efficient inverse modelling of Barotropic Ocean tides. Journal of Atmospheric Oceanic Technology, 19(2), 183-204.

English, L. (2003, July). Plain English about information quality: defining and measuring accuracy. DM Review. Retrieved from http://www.information-management.com/issues/20030701/6954-1.html

Entertainment Technology Centre and Carnegie Mellon University. (2005). Hazmat Hotzone. Retrieved from http://www.etc.cmu.edu/projects/hazmat_2005/

ESRI. (2010a). ArcGIS Explorer. Retrieved from http://www.esri.com/software/arcgis/explorer/index.html 
ESRI. (2010b). ArcScene. Retrieved from

http://webhelp.esri.com/arcgisdesktop/9.2/index.cfm?TopicName=ArcScene 3

D_display_environment

ESRI. (2010c). ArcGIS Tracking Analyst. Retrieved from http://www.esri.com/software/arcgis/extensions/trackinganalyst/index.html

ETC Simulation. (2010). Advanced Disaster Management Simulator (ADMS). Retrieved from http://trainingfordisastermanagement.com/

Federal Emergency Management Agency. (2008). IS-120.A An introduction to exercises. Retrieved from http://vem.vermont.gov/sites/vem/files/IS_120A_Level_I.pdf

Franklin, R. (2007). Technical advances for digital earth. Retrieved from http://www.imagingnotes.com/go/newsletter.php?mp_id=107

Fuhrmann, S., \& Pike, W. (2004). User-centred design of collaborative geo-visualization tools. In J. Dykes, A. M. MacEachren \& M. J. Kraak (Eds.), Exploring Geovisualization (pp. 591-609). Elsevier.

GarageGames. (2010a). Networking. Retrieved from http://docs.garagegames.com/tgea/official/content/documentation/Engine\%20O verview/Networking.html

GarageGames. (2010b). Torque Game Engine Advanced. Retrieved from http://docs.garagegames.com/tgea/official/

Gärtner, M., Seidel, I., Froschauer, J., \& Berger, H. (2010). The formation of virtual organizations by means of electronic institutions in a 3D e-tourism environment. Information Sciences, 180(17), 3157- 3169.

Gladstone USA. (2010). Cross platform data integration technology. Retrieved from http://www.gladstoneusa.com/cross-platform-data

Glazier, L. (2008, September 12). Lost lives remembered during 9/11 ceremony. The Rocket. Retrieved from http://www.theonlinerocket.com/news/lost-lives-remembered-during-9-11-cere mony-1.2333384

Goldsberry, K. (2008, May). Geovisualization of automobile congestion. Paper presented at Geovisualization of Dynamics, Movement, and Change Workshop at the AGILE 2008 Conference, Girona, Spain. 
Goodchild, M. (1992). Geographical information science. International Journal of Geographic Information Systems, 6(1), 31-45.

Google. (2010). Google Earth. Retrieved from http://www.google.com/earth/index.html

GoogleEarthHacks. (2010). Weather: real-time conditions. Retrieved from http://www.gearthhacks.com/dlcatn45p2/Weather:-Real-time-Conditions.htm

Government of British Columbia. (2010). Data quality. Retrieved from http://www.cio.gov.bc.ca/other/daf/IRM_Glossary.htm

Greenhalgh, C., Purbrick, J., Benford, S., Craven, M. P., Drozd, A., \& Taylor, I. (2000, October). Temporal links: recording and replaying virtual environments. Paper presented at the $8^{\text {th }}$ ACM International Conference on Multimedia, New York, USA.

Haddow, G., \& Bullock, J. (2003). Introduction to emergency management. Amsterdam: Butterworth-Heinemann.

Hedge, V. S., Shalini, G., Nayak, S., \& Rajawat, A. S. (2008). Nearshore coastal process between Karwar and Bhatal, central west coast of India: implications for pollution dispersion. In S. Nayak \& S. Zlatanova (Eds.), Remote Sensing and GIS Technologies for Monitoring and Prediction of Disasters (pp. 101-119). Berlin: Springer-Verlag.

Heywood, I., Cornelius, S., \& Carver, S. (1998). An introduction to Geographical Information Systems. New Jersey: Addison Wesley Longman.

Hofschreuder, J. (2004). Visual Criteria in Detail. (Minor Thesis). Wageningen University, Wageningen, Netherlands.

Huxley, J. (2009, February 11). Horrific, but not the worst we've suffered. Sydney Morning Herald. Retrieved from http://www.smh.com.au/national/horrific-but-not-the-worst-weve-suffered-2009 $\underline{0210-83 \mathrm{ib} . h t m l}$

IZE Ltd. (2010). OpenMaps. Retrieved from http://izeize.com/openmaps/

Jain, S., \& McLean, C. R. (2003). Modelling and simulation for emergency response: workshop report, standards and tools. Darby, PA: Diane Pub Co.

Jirka, S., Bröring, A., \& Stasch, C. (2009, June). Applying OGC Sensor Web 
Enablement to risk monitoring and disaster management. Paper presented at Workshop on "Sensor Web Enablement: Strengthening the SDI" at GSDI 11 World Conference, Rotterdam, Netherlands.

Johnson, R. (2000). GIS technology for disasters and emergency management. Retrieved from http://www.esri.com/library/whitepapers/pdfs/disastermgmt.pdf

Jonas, D. (2004, April 26). SW courts corp. clients: Swabiz portal pushes channel segmentation. Business Travel News. Retrieved from http://www.fornova.net/documents/www-businesstravelnews-com.pdf

Kanjo, E., Benford, S., Paxton, M., Chamberlain, A., Fraser, D. S., Woodgate, D., ...Woolard, A. (2007). MobGeoSen: facilitating personal geosensor data collection and visualization using mobile phones. Personal and Ubiquitous Computing, 12(8), 599-607.

Kelly, H., Blackwood, V., Roper, M., Higgins, G., Klein, G., Tyler, J., ...Squire, K. (2002). Training technology against terror: using advanced technology to prepare America's emergency medical personnel and first responders for a weapon of mass destruction attack. Washington: Federation of American Scientists.

Kim, D. J., Moon, W. M., Hwang, J. H., \& Kim Y. S. (2010, July). Application of KOMPSAT-5 data for emergent oil spill monitoring. Paper presented at 2010 IEEE International Geoscience and Remote Sensing Symposium, Honolulu, Hawaii, USA.

Kim, S., Robson, C., Zimmerman, T., Pierce, J., \& Haber, E. M. (2011, May). Creek watch: pairing usefulness and usability for successful citizen science. Paper presented at 2011 ACM Computer Human Interaction Conference, Vancouver, Canada.

Kitware, Inc. (2010). Visualization Toolkit (VTK). Retrieved from http://www.vtk.org/

Kokla, M., \& Kavouras, M. (2001). Fusion of top-level and geographical domain ontologies based on context formation and complementarity. International Journal of Geographical Information Science, 15(7), 679-687.

Kolbe, T. H., Gröger, G., \& Plümer, L. (2008). CityGML - 3D city models and their potential for emergency response. In S. Zlatanova \& J. Li (Eds.), Geospatial Information Technology for Emergency Response (pp. 257-274). London: Taylor \& Francis. 
Kwan, M. P., \& Lee, J. (2005). Emergency response after 9/11: the potential of real-time 3D GIS for quick emergency response in micro-spatial environments. Computers, Environment and Urban Systems, 29(2), 93-113.

Lahtinen, J. (2004). Radiation monitoring strategy: factors to be considered. Radiation Protection Dosimetry, 109(1-2), 79-82.

Land Trust GIS. (2010). ESRI - ArcScene Software. Retrieved from http://landtrustgis.org/

Langran, G., \& Chrisman, N. R. (1988). A framework for temporal geographic information. Cartographica, 25(3), 1-14.

Lee, J. (2007). A three-dimensional navigable data model to support emergency response in microspatial built-environments. Annals of the Association of American Geographers, 97(3), 512-529.

Lee, J., \& Zlatanova, S. (2008). A 3D data model and topological analyses for emergency response in urban areas. In S. Zlatanova \& J. Li (Eds.), Geospatial Information Technology for Emergency Response (pp. 143-168). London: Taylor \& Francis.

Lienert, C., Kunz, M., Weingartner, R., \& Hurni, L. (2010). Monitoring and comparing: a cartographic web application for real-time visualization of hydrological data. In M. Konecny, S. Zlatonova \& T. Bandrova (Eds.), Geographic Information and Cartography for Risk and Crisis Management (pp. 409-424). Berlin: Springer-Verlag.

Lin, M., Rowaihy, H., Bolbrock, T., Cao, G., \& Porta, T. L. (2008, December). Data collection using RFID and a mobile reader. Paper presented at 2008 IEEE GLOBECOM Global Communications Conference, New Orleans, LA.

Linden Research, Inc. (2010). Second Life. Retrieved from http://secondlife.com/

Louka, M. N., \& Balducelli, C. (2001, June). Virtual reality tools for emergency operation support and training. Paper presented at $8^{\text {th }}$ World Conference on Emergency Management (TIEMS 2001), Oslo, Norway.

Lyard, F., Lefevre, F., Letellier, T., \& Francis, O. (2006). Modelling the global ocean tides: modern insights from FES2004. Ocean Dynamics, 56(5-6), 394-415.

Madey, G., Szabó, G., \& Barabási, A-L. (2006). Wiper: the integrated wireless phone based emergency response system. In V. N. Alexandrov, G. D. van Albada, P. M. A. Sloot \& J. Dongarra (Eds.), Computational Science - ICCS 2006: $6^{\text {th }}$ 
International Conference, Reading, UK, May 28-31, 2006. Proceedings, Part III (Lecture Notes in Computer Science, Volume 3993) (pp. 417-424). Berlin: Springer-Verlag.

Magenes, G., Curone, D., Caldani, L., \& Secco, E. L. (2010, September). Fire fighters and rescuers monitoring through wearable sensors: the ProeTEX project. Paper presented at 2010 Annual International Conference of the IEEE Engineering in Medicine and Biology Society, Buenos Aires, Argentina.

Manganas, A., Tsiknakis, M., Leisch, E., Ponder, M., Molet, T., Herbelin, B., ... Schenone, A. (2004). The JUST VR tool: an innovative approach to training personnel for emergency situations using virtual reality techniques. Journal on Information Technology in Healthcare, 2(6), 399-412.

Mantovani, G., Gamberini, L., Martinelli, M., \& Varotto, D. (2001). Exploring the suitability of virtual environments for safety training: signals, norms and ambiguity in a simulated emergency escape. Cognition, Technology \& Work, $3(1), 33-41$.

Markovic, N., Stanimirovic, A., \& Stoimenov, L. (2009, June). Sensor web for river water pollution monitoring and alert system. Paper presented at $12^{\text {th }}$ AGILE International Conference on Geographic Information Science, Leibniz Universität, Hannover, Germany.

McGrath, D., \& Hill, D. (2004, October). UnrealTriage: A game-based simulation for emergency response. Paper presented at the Huntsville Simulation Conference 2004, Huntsville, AL.

Merrill, D. (2006). Mashups: the new breed of web app. Retrieved from http://www.ibm.com/developerworks/xml/library/x-mashups/index.html

Meyer, M. (2006). An extensible weather system: a focus on "C-Style scripting". Retrieved from http://matt-meyer.com/C-Style\%20Script/Weather\%20System\%20-\%20C\%20S tyle\%20Scripting.doc

Miao, Y., \& Haake, J. M. (2001, January). Supporting problem based learning by a Collaborative Virtual Environment: a cooperative hypermedia approach. Paper presented at $34^{\text {th }}$ Annual Hawaii International Conference on System Sciences (HICSS-34), Maui, Hawaii.

Moallemi, M., Jafer, S., Ahmed, A. S., \& Wainer, G. A. (2011, April). Interfacing DEVS and visualization models for emergency management. Paper presented at 2011 Spring Simulation Conference (SpringSim11), Boston, MA. 
Monahan, C., Mustanski, B., \& Neuner, J. (2009). Using Collaborative Virtual Environments in preparedness and emergency response planning. Retrieved from http://www.sph.umn.edu/research/u-seee/individualprojects/virtualenvironment $\underline{\text { s.asp/ }}$

Murakami, Y., Sugimoto, Y., \& Ishida, T. (2005, July). Modelling human behaviour for virtual training systems. Paper presented at $20^{\text {th }}$ National Conference on Artificial Intelligence (AAAI-05), Pittsburgh, Pennsylvania.

Nanyang Technological University. (2010). COSMOS: crowd simulations for military operations. Retrieved from http://cosmos.ntu.edu.sg/

NASA. (2010). NASA World Wind. Retrieved from http://worldwind.arc.nasa.gov/java/

Nasui, D., Oana, F. A., \& Sgarciu, V. (2010, May). Wireless monitoring system in a safe city with safe mobile. Paper presented at 2010 International Joint Conference on Computational Cybernetics and Technical Informatics (ICCC-CONTI), Timisoara, Romania.

Negishi, M. (2011, April 12). Japan raises nuclear crisis severity to highest level. Reuters. Retrieved from http://www.reuters.com/article/2011/04/12/japan-severity-idUSTKE006357201 $\underline{10412}$

NOAA. (2010a). NOAA's National Weather Service. Retrieved from http://www.weather.gov/

NOAA. (2010b). NOAA's National Ocean Service. Retrieved from http://oceanservice.noaa.gov/

O'Connor, A. (2007). Automatic virtual environments from spatial information and models. (Doctoral dissertation). The University of Melbourne, Melbourne, Australia.

O'Connor, A., Stock, C., \& Bishop, I. (2005, December). SIEVE: An online collaborative environment for visualising environmental model outputs. Paper presented at International Congress on Modelling and Simulation, Melbourne, Australia.

Open Geospatial Consortium. (2010). Sensor Web Enablement. Retrieved from http://www.opengeospatial.org/projects/groups/sensorweb 
Open Source Geospatial Foundation. (2010). GRASS GIS. Retrieved from http://grass.osgeo.org/

OpenStreetMap. (2010). OpenStreetMap. Retrieved from http://www.openstreetmap.org/

Oracle. (2010a). Enterprise data Mashup services. Retrieved from http://wiki.open-esb.java.net/Wiki.jsp?page=EnterpriseDataMashup

Oracle. (2010b). Java servlet technology. Retrieved from http://www.oracle.com/technetwork/java/javaee/servlet/index.html

Oracle. (2010c). MySQL. Retrieved from http://www.mysql.com/

Pan, M., Tsai, C., \& Tseng, Y. (2006). Emergency guiding and monitoring applications in indoor 3D environments by wireless sensor networks. International Journal of Sensor Networks, 1(1-2), 2-10.

Pečiva, J. (2007). Active transactions in Collaborative Virtual Environments. (Doctoral dissertation). Brno University of Technology, Brno, Czech Republic.

Pettersson, L. W., Spak, U., \& Seipel, S. (2004). Collaborative 3D visualizations of geo-spatial information for command and control. In S. Seipel (Ed.), The Annual SIGRAD Conference, Special Theme - Environmental Visualization (pp. 41-47). Linköping: Linköping University Electronic Press.

Peuquet, D. J., \& Duan, N. (1995). An event-based spatiotemporal data model (ESTDM) for temporal analysis of geographical data. International Journal of Geographical Information Systems, 9(1), 7-24.

Ponder, M., Herbelin, B., Molet, T., Schertenlieb, S., Ulicny, B., Papagiannakis, G., ...Thalmann, D. (2003, May). Immersive VR decision training: telling interactive stories featuring advanced virtual human simulation technologies. Paper presented at EGVE '03 the Workshop on Virtual Environments, Zurich, Switzerland.

PRESAGIS. (2010a). Swiss terrain. Retrieved from http://www.presagis.com/resources/gallery/

PRESAGIS. (2010b). Vega Prime. Retrieved from http://www.presagis.com/products_services/products/ms/visualization/vega_pri $\underline{\mathrm{mel}}$ 
Public Safety Canada. (2010). Emergency management planning guide 2010-2011. Retrieved from http://www.publicsafety.gc.ca/prg/em/emp/_fl/emp-gd-2010-11-e.pdf

Pundt, H. (2008). The semantic mismatch as limiting factor for the use of geospatial information in disaster management and emergency response. In S. Zlatanova \& J. Li (Eds.), Geospatial Information Technology for Emergency Response (pp. 243-256). London: Taylor \& Francis.

Ramsey, M., Dehn, J., Wessels, R., Byrnes, J., Duda, K., Maldonado, L., \& Dwyer, J. (2004, December). The ASTER emergency scheduling system: a new project linking near-real-time satellite monitoring of disasters to the acquisition of high-resolution remote sensing data. Paper presented at American Geophysical Union, Fall Meeting 2004, San Francisco, CA.

Rao, R. R., Eisenberg, J., \& Schmitt, T. (2007). Improving disaster management: The role of IT in mitigation, preparedness, response, and recovery. Washington, DC: National Academies Press.

Raper, J., \& Livingstone, D. (1995). Development of a geomorphological spatial model using object-oriented design. International Journal of Geographical Information Systems, 9(4), 359-383.

Rizzo, A., \& Kim, G. J. (2005). A SWOT analysis of the field of virtual reality rehabilitation and therapy. Presence, 14(2), 119-146.

Ross, L., Bolling, J., Döllner, J., \& Kleinschmit, B. (2009). Enhancing 3D city models with heterogeneous spatial information: towards 3D land information systems. In M. Sester, L. Bernard \& V. Paelke (Eds.), Advances in GIScience, Proceedings of the $12^{\text {th }}$ AGILE Conference (Lecture Notes in Geoinformation and Cartography) (pp. 113-133). Berlin: Springer-Verlag.

Santoro, A., \& Quaglia, F. (2006). Transparent state management for optimistic synchronization in the High Level Architecture. Journal of Simulation, 82(1), $5-20$.

Schoenharl, T., Madey, G., Szabó, G., \& Barabási, A-L. (2006, May). WIPER: A multi-agent system for emergency response. Paper presented at the 3rd International ISCRAM Conference, Newark, NJ.

Schrenk, M. (2007). Webbots, spiders, and screen scrapers: a guide to developing Internet agents with PHP/CURL. San Francisco, CA: No Starch Press.

Schrenk, M., \& Pozarek, W. (2007, May). Centrope MAP: Combining X-Border 
Information from Multiple Sources for Planning Purposes. Paper presented at the Third International Workshop on Information Fusion and Geographical Information Systems, St. Petersburg, Russia.

Shao, Y., Gong, H., Wang, S., Zhang, F., \& Tian, W. (2009, May). Multi-source SAR remote sensing data for emergency monitoring to Wenchuan earthquake damage assessment. Paper presented at 2009 Joint Urban Remote Sensing Event, Shanghai, China.

Sharkawi, K. H., Ujang, M. U., \& Abdul-Rahman, A. (2008). 3D navigation system for virtual reality based on 3D game engine. In S. Nayak (Ed.), The International Archives of the Photogrammetry Remote Sensing and Spatial Information Sciences, ISPRS Congress Beijing 2008, Proceeding of Commission IV (pp 513-518). Beijing: ISPRS.

Shepard, D. (1968, August). A two-dimensional interpolation function for irregularly-spaced data. Paper presented at 1968 ACM National Conference, New York.

Shiode, N., \& Yin, L. (2008). Spatial temporal visualization of built environments. In K. S. Hornsby \& M. Yuan (Eds.), Understanding Dynamics of Geographic Domains (pp.133-150). Boca Raton, FL: CRC Press.

Smith, T. M., \& Lakshmanan, V. (2011). Real-time, rapidly updating severe weather products for virtual globes. Computers \& Geosciences, 37(1), 3-12.

SourceForge. (2010). Htmlcxx. Retrieved from http://htmlcxx.sourceforge.net/

SpatialVision. (2010). EmerGeo - Emergency Management GIS. Retrieved from http://www.spatialvision.com.au/index.php/software-a-data/emergeo.html

Stock, C., Bishop, I. D., O’Connor, A., Chen, A. T., Pettit, C. J., \& Aurambout, J-P. (2008). SIEVE: collaboration decision-making in an immersive online environment. Cartography and Geographic Information Science, 35(2), 133-144.

Stock, C., Bishop, I. D., Sharma, S., \& Chen, H. (2010, May). Online collaboration in Australian virtual landscapes. Paper presented at Digital Landscape Architecture 2010, Aschersleben, Germany.

Surendranath, M., Ghosh, S., Ghoshal, T. B., \& Rajendran, N. (2008). Landslide hazard zonation in Darjeeling Himalayas: a case study on integration of IRS and SRYM data. In S. Nayak \& S. Zlatanova (Eds.), Remote Sensing and GIS Technologies for Monitoring and Prediction of Disasters (pp.121-135). Berlin: 
Springer-Verlag.

Tanasescu, V., Gugliotta, A., Domingue, J., Davies, R., Gutiérrez-Villarías, L., Rowlatt, M., ...Stinčić, S. (2006). A semantic web services GIS based emergency management application. In I. Cruz, S. Decker, D. Allemang, C. Preist, D. Schwabe, P. Mika, ...L. Aroyo (Eds.), The Semantic Web - ISWC 2006 (Lecture Notes in Computer Science, Volume 4273) (pp. 959-966). Berlin: Springer-Verlag.

Tatalovich, Z. (2005). A comparison of Thiessen-polygon, Kriging, and spline models of $U V$ exposure. Retrieved from http://www.ucgis.org/summer2005/studentpapers/tatalovich.pdf

The New York Times. (2009, May 6). Sichuan earthquake. Retrieved from http://topics.nytimes.com/topics/news/science/topics/earthquakes/sichuan_provi nce_china/index.html

The Weather Channel, LLC. (2010a). The Weather Channel. Retrieved from http://www.weather.com/

The Weather Channel, LLC. (2010b). WeatherBonk. Retrieved from http://www.weatherbonk.com/

The Weather Company. (2010). WeatherZone. Retrieved from http://www.weatherzone.com.au/

Thiessen, A. H. (1911). Precipitation averages for large areas. Monthly Weather Review, 39(7): 1082-1084.

Tiede, D., \& Lang, S. (2010). Analytical 3D views and virtual globes - scientific results in a familiar spatial context. Journal of Photogrammetry and Remote Sensing, 65(3), 300-307.

Tomaszewski, B. (2011). Situation awareness and virtual globes: applications for disaster management. Computers and Geosciences, 37(1), 86-92.

Tominski, C., Schulze-Wollgast, P., \& Schumann, H. (2005, July). 3D information visualization for time dependent data on maps. Paper presented at Ninth International Conference on Informational Visualization, London, UK.

Transportation Research Board of the National Academies. (2006). Guidelines for transportation emergency training exercises. Retrieved from http://onlinepubs.trb.org/onlinepubs/nchrp/nchrp_rpt_525v9.pdf 
Tsai, V. J. D. (1993). Fast topological construction of Delaunay triangulations and Voronoi diagrams. Computer and Geosciences, 19 (10), 1463-1474.

Tseng, J. M., Shu, C. M., Horng, J. J., Kuan, C. M., \& Hsu, H. I. (2007). Planning an emergency response centre in southern Taiwan Science Park. Process Safety and Environmental Protection, 85(2), 125-132.

University of the Aegean. (2010). Marine traffic. Retrieved from http://www.marinetraffic.com/ais/

Upadhyay, R., Pringle, G., Beckett, G., Potter, S., Han, L., Welch, S., ...Torero, J. (2009). An architecture for an integrated fire emergency response system for the built environment. In B. Karlsson (Ed.), Fire Safety Science - Proceedings of the Ninth International Symposium (pp. 427-438). London: International Association for Fire Safety Science.

U.S. Agency for International Development. (2006). After-action review technical guidance. Retrieved from http://pdf.usaid.gov/pdf_docs/PNADF360.pdf

U.S. Department of Homeland Security. (2006). National planning scenarios. Retrieved from https://www.llis.dhs.gov/docdetails/details.do?contentID=13712

U.S. Department of Homeland Security. (2007). National preparedness guidelines. Retrieved from http://www.dhs.gov/xlibrary/assets/National_Preparedness_Guidelines.pdf

U.S. Department of Transportation. (1998). Critical incident management guidelines. Retrieved from http://transit-safety.fta.dot.gov/publications/Emergency/CriticalIncidents/PDF/C ritical_Incident_Management_Guidelines.pdf

VanDaniker, M. (2009, August). Visualizing real-time and archived traffic incident data. Paper presented at $10^{\text {th }}$ IEEE International Conference on Information Reuse \& Integration, Las Vegas, NV.

Van Ruijven, T. (2011, May). Serious Games as Experiments for Emergency Management Research: A Review. Paper presented at the $8^{\text {th }}$ International ISCRAM Conference, Lisbon, Portugal.

Vesseltracker. (2010). Vesseltracker. Retrieved from http://www.vesseltracker.com/en/Home.html

Virginia Department of Health. (2011). Public heath preparedness training program and exercise guidance. Retrieved from 
http://www.vdh.state.va.us/oep/pdf/vdh_wdp.pdf

Virginia Institute of Marine Science. (2010). Tide prediction - HAMELS vs. NOAA/NOS. Retrieved from http://web.vims.edu/physical/research/TCTutorial/tidepredict.htm

VSTEP. (2010). RescueSim. Retrieved from http://www.rescuesim.com/

Wahba, G. (1990). Spline models for observational data. Philadelphia: Society for Industrial and Applied Mathematics.

Walton, G. H., Patton, R. M., \& Parsons, D. J. (2001, December). Usage testing of military simulation systems. Paper presented at 2001 Winter Simulation Conference, Arlington, VA.

Wang, X., Turner, S. J., Low, Y. K., \& Gan, B. P. (2004, May). Optimistic synchronization in HLA based distributed simulation. Paper presented at the $18^{\text {th }}$ Workshop on Parallel and Distributed Simulation, Kufstein, Austria.

Wickler, G., Potter, S., Tate, A., \& Hansberger, J. (2011, May). The Virtual Collaboration Environment: New Media for Crisis Response. Paper presented at the $8^{\text {th }}$ International ISCRAM Conference, Lisbon, Portugal.

Wikicrimes. (2010). Wikicrimes. Retrieved from http://www.wikicrimes.org/main.html

Willy Online Pty Ltd. (2010). WillyWeather. Retrieved from http://www.willyweather.com.au

Wimmer, M., \& Wonka, P. (2003, June). Rendering time estimation for real-time rendering. Paper presented at the $14^{\text {th }}$ Eurpgraphics Workshop on Rendering Techniques, Leuven, Belgium.

Wisconsin Department of Transportation. (2008). Emergency traffic control and scene management guidelines. Retrieved from http://www.dot.wisconsin.gov/travel/stoc/docs/emer-tc-sm-guidelines.pdf

Wolf, H. (1963). Geometric connection and re-orientation of three-dimensional triangulation nets. Bulletin Géodésique, 68(1), 165-169.

Worboys, M. F. (1992, August). A model for spatio-temporal information. Paper presented at Proceedings of the $5^{\text {th }}$ International Symposium on Spatial Data Handling, Charleston, South Carolina, USA. 
Wright, T. E., \& Madey, G. (2008, May). A prototype virtual emergency operations center using a Collaborative Virtual Environment. Paper presented at $5^{\text {th }}$ International ISCRAM Conference, Washington, DC.

Wu, P., Ott, T., \& Miller, C. (2009, March). Evaluating the effects of culture and etiquette on human-computer interaction and human performance. Paper presented at AAAI 2009 Spring Symposium, Palo Alto, CA.

Wyld, D. C. (2010). A virtual explosion or SNAFU is always better than a real one: exploring the use of virtual worlds for simulation and training... and developing the leaders of tomorrow. In M. Iskander, V. Kapila \& M. A. Karim (Eds.), Technological Developments in Education and Automation (pp. 73-78). New York, NY: Springer-Verlag.

Xu, W., \& Zlatanova, S. (2007). Ontologies for disaster management response. In J. Li, S. Zlatanova \& A. Fabbri (Eds.), Geomatics Solutions for Disaster Management (pp. 185-200). New York, NY: Springer-Verlag.

Yahoo! Inc. (2010). Yahoo! Weather. Retrieved from http://weather.yahoo.com/

You, X., Liu, Y., \& Xu, W. (2010, July). The design of a remote monitoring system based on Java. Paper presented at $20103^{\text {rd }}$ IEEE International Conference on Computer Science and Information Technology (ICCSIT), Chengdu, China.

Yu, L., Lu, A., Ribarsky, W., \& Chen, W. (2010). Automatic animation for time-varying data visualization. Computer Graphics Forum, 29(7), 2271-2280.

Yu, L., Wang, N., \& Meng, X. (2005, September). Real-time forest fire detection with wireless sensor networks. Paper presented at International Conference on Wireless Communications, Networking and Mobile Computing, Wuhan, China.

Zarraonandia, T., Vargas, M. R., Díaz, P., \& Aedo, I. (2009). A virtual environment for learning airport emergency management protocols. In J. A. Jacko (Ed.), Human-Computer Interaction: Ambient, Ubiquitous and Intelligent Interaction13th International Conference, HCI International 2009, San Diego, CA, USA, July 19-24, 2009, Proceedings, Part III (Lecture Notes in Computer Science, Volume 5612) (pp. 228-235). Berlin: Springer-Verlag.

Zeng, Y., Sreenan, C. J., Sitanayah, L., Xiong, N., Park, J. H., \& Zheng, G. (2011). An emergency-adaptive routing scheme for wireless sensor networks for building fire hazard monitoring. Sensors, 11(3), 2899-2919.

Zerger, A., \& Smith, D. I. (2003). Impediments to using GIS for real-time disaster decision support. Computers, Environment and Urban Systems, 27(2), 123-141. 
Zhao, Q. S., Huang, Q., Guo, J. M., Wen, R., \& Zhong, S. (2007). A model of spatial data integration interoperability on Oracle Spatial. In J. Li, S. Zlatanova \& A. Fabbri (Eds.), Geomatics Solutions for Disaster Management (pp. 289-303). New York, NY: Springer-Verlag.

Zhou, G. Q. (2009). Near real-time orthorectification and mosaic of small UAV video flow for time-critical event response. IEEE Transactions on Geoscience and Remote Sensing, 47(3), 739-747.

Zhu, Y. C., Tang, M., \& Du, M. (2010). An emergency monitoring about sudden water pollution accident in drinking water sources. In W. Li (Ed.), Proceedings 2010 IEEE International Conference on Emergency Management and Management Sciences (pp. 99-101). Beijing: IEEE Press.

Zlatanova, S., Fabbri, A. G., \& Li, J. (2005, March). Geo-information for disaster management: large scale 3D data needed by urban areas. GIM International, 19(3), 10-13.

Zlatanova, S., \& Nayak, S. (2008). Introduction. In: S. Nayak \& S. Zlatanova (Eds.), Remote Sensing and GIS Technologies for Monitoring and Prediction of Disasters (pp. 1-7). Berlin: Springer-Verlag. 



\section{Appendices}

\section{Appendix 1: Weather Codes and Conditions}

\begin{tabular}{|c|c|}
\hline Code & Description \\
\hline 0 & tornado \\
\hline 1 & tropical storm \\
\hline 2 & hurricane \\
\hline 3 & severe thunderstorms \\
\hline 4 & thunderstorms \\
\hline 5 & mixed rain and snow \\
\hline 6 & mixed rain and sleet \\
\hline 7 & mixed snow and sleet \\
\hline 8 & freezing drizzle \\
\hline 9 & drizzle \\
\hline 10 & freezing rain \\
\hline 11 & showers \\
\hline 12 & showers \\
\hline 13 & snow flurries \\
\hline 14 & light snow showers \\
\hline 15 & blowing snow \\
\hline 16 & snow \\
\hline 17 & hail \\
\hline 18 & sleet \\
\hline 19 & dust \\
\hline 20 & foggy \\
\hline 21 & haze \\
\hline 22 & smoky \\
\hline 23 & blustery \\
\hline 24 & windy \\
\hline 25 & cold \\
\hline 26 & cloudy \\
\hline 27 & mostly cloudy (night) \\
\hline
\end{tabular}




\begin{tabular}{|l|l|}
\hline 28 & mostly cloudy (day) \\
\hline 29 & partly cloudy (night) \\
\hline 30 & partly cloudy (day) \\
\hline 31 & clear (night) \\
\hline 32 & sunny \\
\hline 33 & fair (night) \\
\hline 34 & fair (day) \\
\hline 35 & mixed rain and hail \\
\hline 36 & hot \\
\hline 37 & isolated thunderstorms \\
\hline 38 & scattered thunderstorms \\
\hline 39 & scattered thunderstorms \\
\hline 40 & scattered showers \\
\hline 41 & heavy snow \\
\hline 42 & scattered snow showers \\
\hline 43 & heavy snow \\
\hline 44 & partly cloudy \\
\hline 45 & thundershowers \\
\hline 46 & snow showers \\
\hline 47 & isolated thundershowers \\
\hline 3200 & not available \\
\hline & \\
\hline 30
\end{tabular}




\section{Appendix 2: SIEVE-VTS Demonstration User Feedback}

\section{SIEVE-VTS Survey}

This questionnaire aims to determine which aspects are of importance in supporting emergency management via Collaborative Virtual Environment. You will be shown different types of scenarios and tools, and asked specific questions. These are intended to help stimulate and structure the discussion.

1) How well does the method deliver the information which has been included in this example? (Please circle the number)

A. Maps using Web feed:

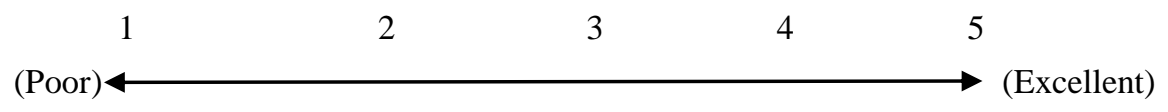

Comments on this method (e.g. merits and drawbacks, range of use):

B. CVE using web feed:

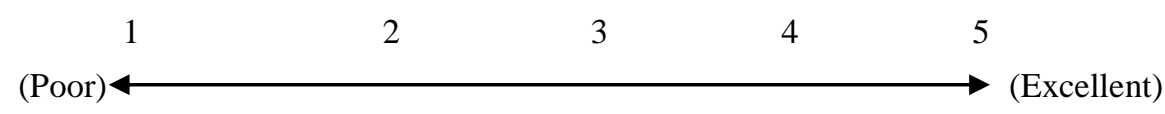

Comments on this method (e.g. merits and drawbacks, range of use):

2) Which direction and how far do you need to go to reach the incident location? And how do you make this estimation? 
3) How useful do you think each of the three tools is when used for emergency management?

$\begin{array}{lccccc}\text { A. COSMOS: } & 1 & 2 & 3 & 4 & 5 \\ & \text { (Not useful) } & & & & \end{array}$

Comments on this method (e.g. Features, merits and drawbacks):

B. Unreal Airport:

$\begin{array}{ccccc}1 & 2 & 3 & 4 & 5 \\ (\text { Not useful }) \longleftrightarrow \text { (Very useful) }\end{array}$

Comments on this method (e.g. Features, merits and drawbacks):

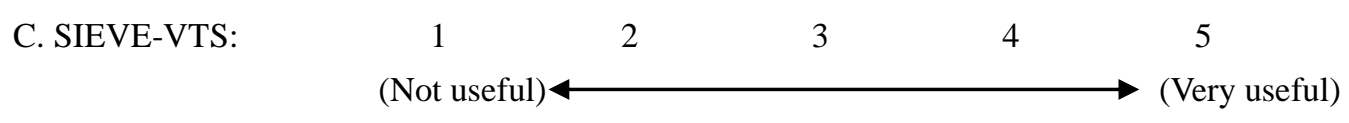

Comments on this method (e.g. Features, merits and drawbacks):

4) What do you think the speed of the ship in this scenario, and how do you make the estimation?

What is the water level do you think at the highest point of the tide, and how do you make the estimation? 
What is visibility level on Sydney Harbour in the foggy condition, and how do you make the estimation?

5) In which field do you think SIEVE-VTS can best be used to support emergency management?

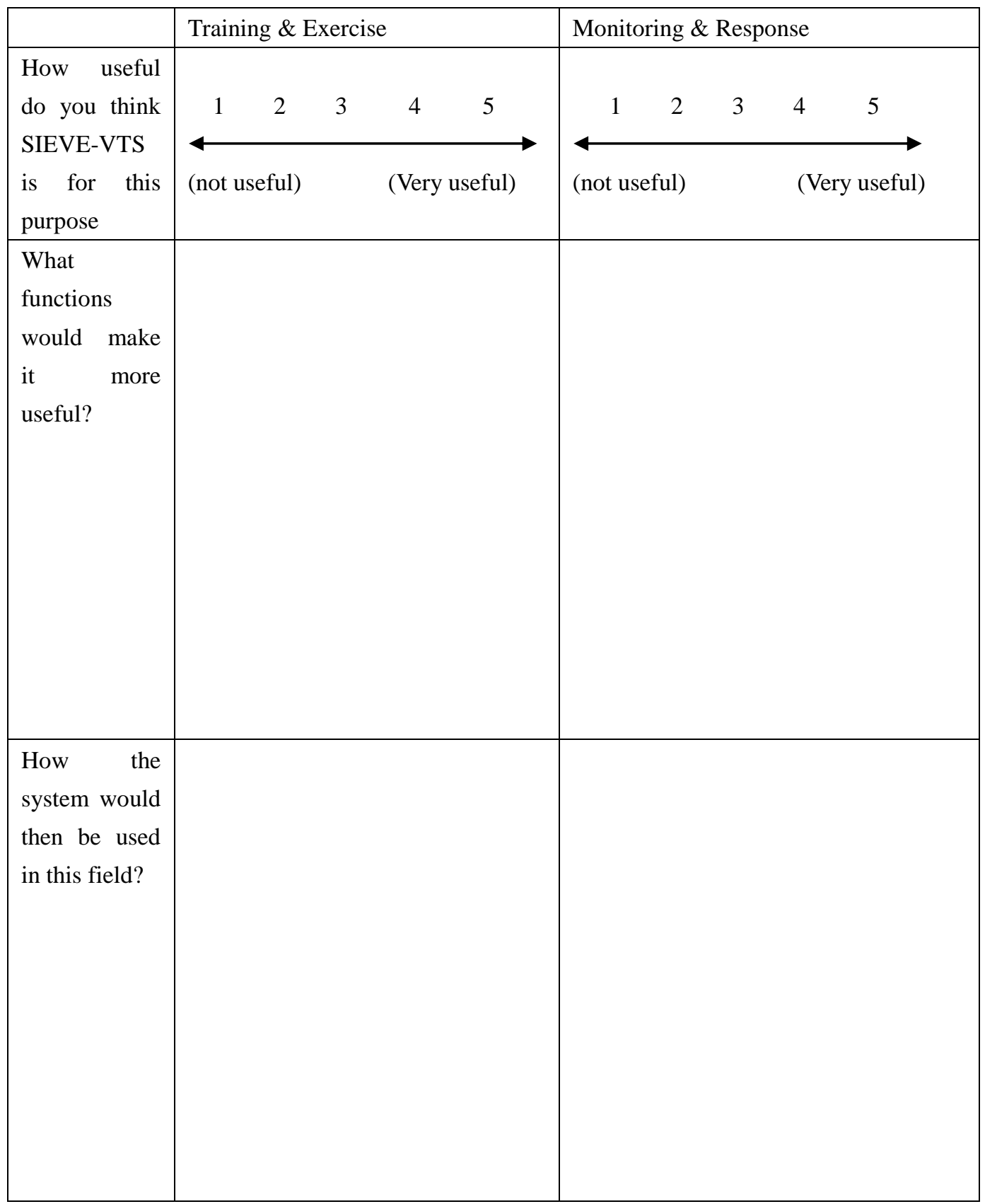


What other field of application is it possible for this system, and how to use it?

6) Additional comments 


\section{Appendix 3: SIEVE-VTS User Documentation}

\section{Introduction}

The user documentation illustrates the usage of SIEVE-VTS (Spatial Information Exploration and Visualisation Environment -Virtual Training System), and real-time data integration module within the system. Specifically, it is designed for DSTO Sydney, and other potential users of this system. This system is developed under the project "Applications of Collaborative Virtual Environments" undertaken by CRC for Spatial Information (CRC-SI), University of Melbourne, DSTO and collaborating partners. SIEVE-VTS is a collaborative virtual environment based on Torque Game Engine Advanced (http://www.torquepowered.com/). Its real-time data integration module can capture, store, and visualise real-time data in a virtual environment. This is potentially useful for virtual military training or emergency response.

\section{SIEVE-VTS Manual}

\subsection{Before starting the game}

In the project you can find 3 folders: SIEVE-VTS, which is the Virtual Training System; database includes sample historical data for emergency training; incident reporter is an application for iPhone which can collect and transfer incident data to a central server.

Before you start the virtual training system, you must make sure that the system has a hardware accelerate card (NVIDIA or ATI is recommended) which has excellent performance on rendering. MySQL database management tool is also required for restoring historical data.

\subsection{Import data into MySQL}

1. Start MySQL Administrator, log in using "root" account;

2. Choose "Catalogs" in the view panel;

3. In schemata panel, right click the mouse and create new schema named "torque";

4. Choose "Restore" in the view panel, on the general tab, click "Open Backup File", and choose "torque 0728 " in the disk;

5. In Target Schema, select "Another schema", then choose "torque", and start restore.

Now you have imported historical data into your own MySQL.

\subsection{SIEVE-VTS basic control}

1. Start SIEVE-VTS by double-clicking SIEVE-VTS.exe in /example/.

2. Click Start Mission... $\rightarrow$ choose example in Select Mission $\rightarrow$ Launch Mission Later you will enter a virtual training world modelled on Sydney Harbour. You can control the avatar or camera by pressing keyboard:

W: Move forward

A: Move left

S: Move backward

D: Move right

SPACE: jump

Alt $+\mathrm{C}$ : toggle avatar/camera view

$E$ : increase field of view 
F7: drop avatar at camera

F8: drop camera at avatar

F11: open world editor

ESC: Quit the scenario

\section{Real-Time Data Integration/Visualisation Module}

The real-time data integration module is designed for integrating real-time data which could potentially be useful for emergency training and response in a virtual environment. Such data include: weather information, tide/wave data, AIS (Automatic Identification System) data, incident location and status. Follow these steps to start the functionality of this module.

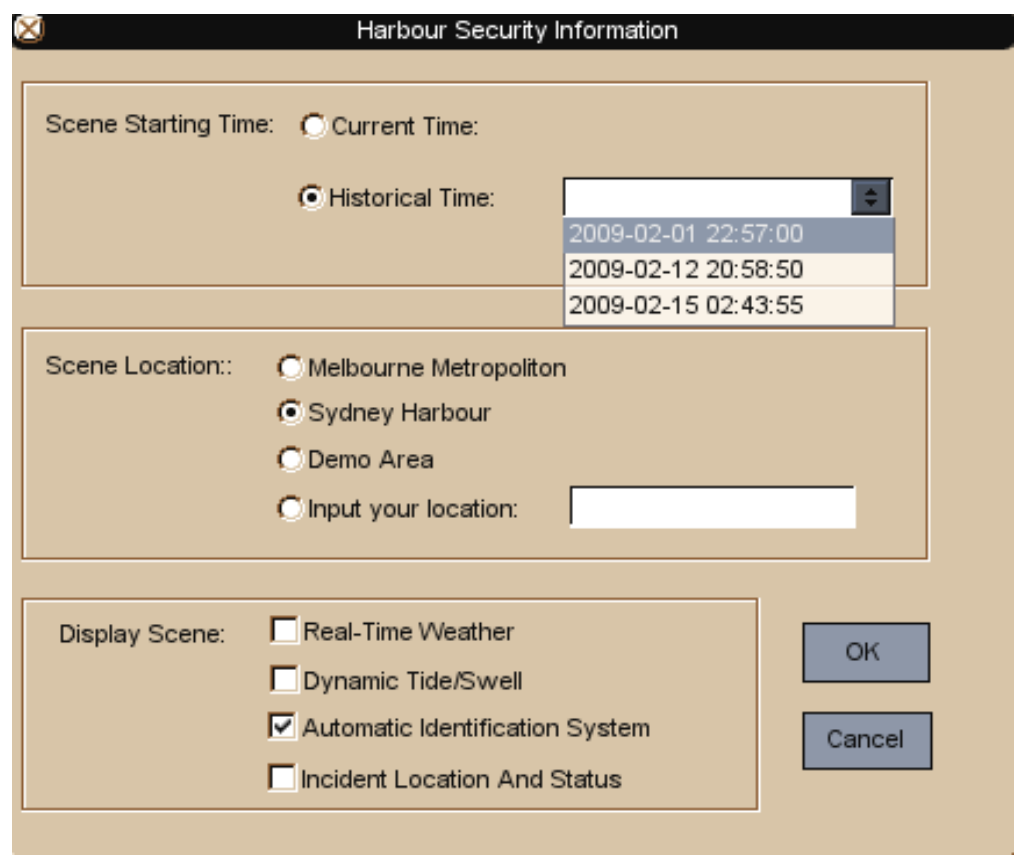

-In the player or camera view, press $\mathrm{Ctrl}+\mathrm{H}$, and Harbour Security Information dialog will pop up.

·In "Scene Starting Time" section, you can either choose "Current Time" or one of "Historical Time" in the popup menu. Accordingly the starting time in the Virtual Training System will be set to the time you chose.

-In "Scene Location" section, you may choose the location from "Melbourne Metropolitan", "Sydney Harbour" or "Demo Area". Information of this area will be loaded from database or online data provider, depending on what you chose in "Scene Starting Time". (Note: Scenario is based on Sydney in the demo; choosing different "Scene Locations" does not change the location of scenario to other place. This function aims at demonstrating different information from various locations (e.g. Melbourne weather), though it does not comply with real situation.)

-In "Display Scene" session, you can select one or more types of scenes to display. These types of data are mentioned at the beginning of this section.

-After you set up all the parameters, click OK and wait for a few seconds. During that time virtual scenes will be loaded, detailed information will be displayed as text in the GUI control as well. 
$\oplus$ Weather Information: displayed on the right part of GUI. It contains information about weather location, wind direction and speed, humidity, visibility, and so forth. You can notice that the wind will affect the cloud moving speed and direction, and basic weather condition such as sunny, cloudy or rainy can be rendered in the virtual world.

ФAIS information: displayed on the left part of GUI. It contains information about ship location and status within that area, such as MMSI of the ship, latitude and longitude of the ship. All the ships available are listed and you can choose a ship by clicking the MMSI of it, the ship chosen is highlighted with a white box around it. All the ships are moving on a track which is modelled using real AIS data, and you can read current time and location of the ship from the information GUI.

$\bigoplus$ Tide/Wave information: displayed on the left part of GUI. It contains information about tide and wave height of current time. Move close to the bank of the sea, you will notice that water level goes up and down regularly. Real-time water level is shown on the GUI and changing all the time. $\oplus$ Incident location and status: Incident location and status information is delivered from iPhone application iReport, which collects and transfers instant data into SIEVE-VTS. Afterwards, these incidents such as fire, explosion or toxic gas leakage could be simulated in the virtual training system.

You can press Ctrl+J to toggle on/off the information GUI.

\section{Incident reporter for iPhone}

Incident reporter is designed for security guard or policeman reporting incident locations and status using iPhone. Since iPhone has been becoming a popular platform, it is convenient for people to collect and distribute real-time data using such a tool.

\subsection{Installation}

Due to development limitation of iPhone, this application cannot be installed on unauthorised systems. However, if you have your provisioning profile, you could just release the application on your iPhone using Xcode.

\subsection{Usage of iReport}




\begin{tabular}{|c|c|c|c|}
\hline $33 G$ & 11:10 AM & $83^{\circ}$ & $\% 3$ \\
\hline \multicolumn{4}{|c|}{ Incident Report } \\
\hline \multicolumn{4}{|c|}{ Location Info } \\
\hline \multicolumn{2}{|c|}{ Current Location } & OFF & \\
\hline Longitude: & \multicolumn{3}{|c|}{ Enter Longitude } \\
\hline Latitude: & \multicolumn{3}{|c|}{ Enter Latitude } \\
\hline Address: & \multicolumn{3}{|c|}{ Enter Address } \\
\hline \multicolumn{4}{|l|}{ Incident Info } \\
\hline \multicolumn{3}{|c|}{ Type: to be set } & $>$ \\
\hline \multicolumn{3}{|c|}{ Size: to be set } & $>$ \\
\hline \multicolumn{3}{|c|}{ Status: to be set } & $>$ \\
\hline
\end{tabular}

1. Click iReport icon on the iPhone touch screen to start the application.

2. Input the location information. You can either use current location (iPhone supports recording current location by embedded GPS) or input longitude and latitude of a certain place.

3. Choose Incident type, size and status from incident info section. Input Personal Info of the reporter.

4. Submit data by clicking the button on the bottom.

By implementing the steps above, you have updated data to a server which stores incident location and status. This is then displayed in the virtual environment.

Note: This user documentation, with the described software (SIEVE-VTS and iReport) including source code were provided with DSTO, Sydney and other potential collaborators of this project. 


\section{University Library}

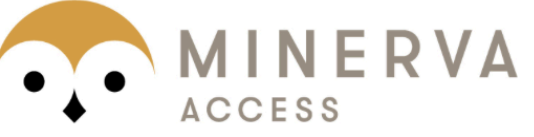

A gateway to Melbourne's research publications

Minerva Access is the Institutional Repository of The University of Melbourne

Author/s:

WANG, PAN

Title:

Real-time data visualisation in collaborative virtual environment for emergency management

Date:

2011

Citation:

Wang, P. (2011). Real-time data visualisation in collaborative virtual environment for emergency management. PhD thesis, Engineering, Department of Infrastructure Engineering, The University of Melbourne.

Persistent Link:

http://hdl.handle.net/11343/37070

\section{Terms and Conditions:}

Terms and Conditions: Copyright in works deposited in Minerva Access is retained by the copyright owner. The work may not be altered without permission from the copyright owner. Readers may only download, print and save electronic copies of whole works for their own personal non-commercial use. Any use that exceeds these limits requires permission from the copyright owner. Attribution is essential when quoting or paraphrasing from these works. 